Keywords carbon, oxalate, formate, nitrate

Retention: permanent

\title{
INTEGRATION OF THE UNCERTAINTIES OF ANION AND TOC MEASUREMENTS INTO THE FLAMMABILITY CONTROL STRATEGY FOR SLUDGE BATCH 8 AT THE DWPF
}

T.B. Edwards

March 2013

Applied Computational Engineering and Statistics Savannah River National Laboratory

Aiken, SC 29808

This document was prepared in conjunction with work accomplished under Contract No. DE-AC09-08SR22470 with the U.S. Department of Energy.

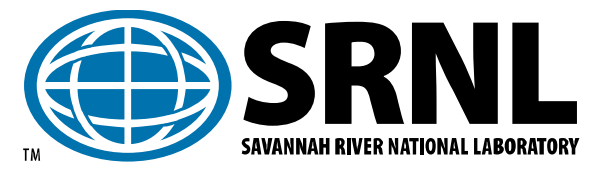


SRNL-STI-2013-00139

Revision 0

\section{DISCLAIMER}

This work was prepared under an agreement with and funded by the U.S. Government. Neither the U.S. Government or its employees, nor any of its contractors, subcontractors or their employees, makes any express or implied: 1. warranty or assumes any legal liability for the accuracy, completeness, or for the use or results of such use of any information, product, or process disclosed; or 2. representation that such use or results of such use would not infringe privately owned rights; or 3. endorsement or recommendation of any specifically identified commercial product, process, or service. Any views and opinions of authors expressed in this work do not necessarily state or reflect those of the United States Government, or its contractors, or subcontractors.

This document was prepared in conjunction with work accomplished under Contract No. DE-AC09-08SR22470 with the U.S. Department of Energy. 
SRNL-STI-2013-00139

Revision 0

\section{REVIEWS AND APPROVALS}

AUTHOR:

T. B. Edwards, Applied Computational Engineering and Statistics Date

Savannah River National Laboratory

\section{TECHNICAL REVIEWER:}

E. P. Shine, Applied Computational Engineering and Statistics Date Savannah River National Laboratory

Technical Reviewer/

Independent Statistical Software Verifier (see Attachment 1)

\section{APPROVERS:}

P. L. Lee, Manager, Applied Computational Engineering and Statistics Date Savannah River National Laboratory

C. C. Herman, Manager, Process Technology Programs

Date

Savannah River National Laboratory

R. T. McNew, Manager, Waste Laboratory Services

Date

Savannah River Remediation

E. J. Freed, Manager, Waste Solidification Engineering

Date

Savannah River Remediation 


\section{EXECUTIVE SUMMARY}

The Savannah River National Laboratory (SRNL) has been working with the Savannah River Remediation (SRR) Defense Waste Processing Facility (DWPF) in the development and implementation of a flammability control strategy for DWPF's melter operation during the processing of Sludge Batch 8 (SB8). SRNL's support has been in response to technical task requests that have been made by SRR's Waste Solidification Engineering (WSE) organization. The flammability control strategy relies on measurements that are performed on Slurry Mix Evaporator (SME) samples by the DWPF Laboratory.

Measurements of nitrate, oxalate, formate, and total organic carbon (TOC) standards generated by the DWPF Laboratory are presented in this report, and an evaluation of the uncertainties of these measurements is provided. The impact of the uncertainties of these measurements on DWPF's strategy for controlling melter flammability also is evaluated. The strategy includes monitoring each SME batch for its nitrate content and its TOC content relative to the nitrate content and relative to the antifoam additions made during the preparation of the SME batch.

A linearized approach for monitoring the relationship between TOC and nitrate is developed, equations are provided that integrate the measurement uncertainties into the flammability control strategy, and sample calculations for these equations are shown to illustrate the impact of the uncertainties on the flammability control strategy. 


\section{TABLE OF CONTENTS}

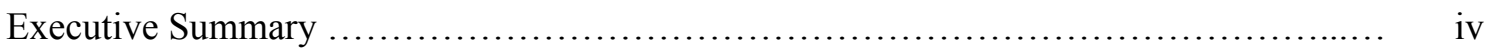

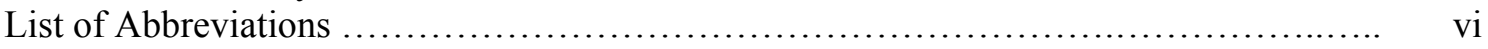

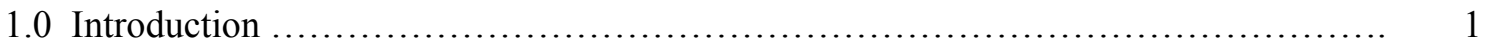

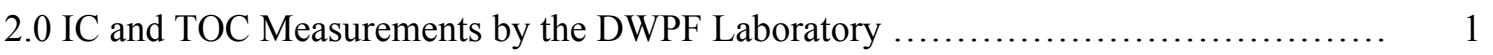

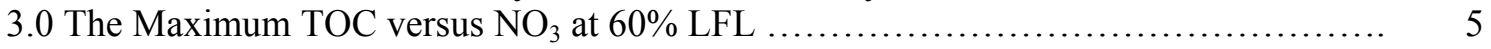

3.1 Assessing the Nitrate Content of the SME .............................. 5

3.2 Controlling Melter Flammability via the TOC to Nitrate Relationship .......... 9

3.3 Meeting the Constraint Imposed by the TOC to Nitrate Relationship ........... 12

3.4 Example Calculations in Meeting the TOC Constraint ........................ 13

4.0 Estimation of the Antifoam Addition .......................................... 15

4.1 Bounding the Total Amount of Antifoam Additions Made to a SME Batch .... 16

4.2 Meeting the Constraint on the Antifoam Addition ......................... 20

4.3 Example Calculations in Meeting the Antifoam Constraint .................. 21

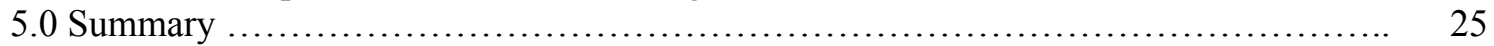

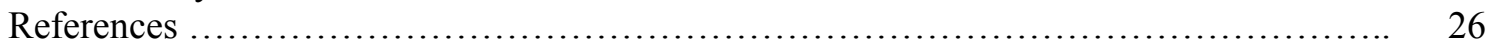

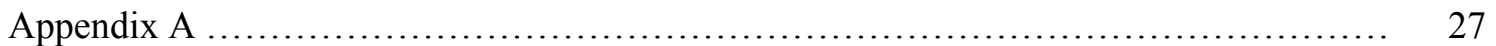

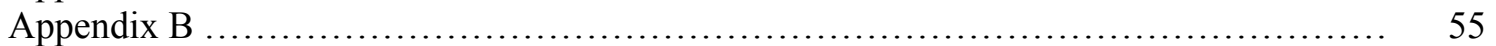

Attachment 1 ................................................................... 56 


\section{LIST OF ABBREVIATIONS}

$\begin{array}{ll}\text { DWPF } & \text { Defense Waste Processing Facility } \\ \text { gal } & \text { gallons } \\ \text { IC } & \text { Ion Chromatography } \\ \text { JMP } & \text { Statistical software package from SAS Institute, Inc. [5] } \\ \text { Lab OPS } & \text { Laboratory Operations } \\ \text { LFL } & \text { Lower Flammability Limit } \\ \text { ppm } & \text { parts per million } \\ \text { SB8 } & \text { Sludge Batch 8 } \\ \text { SME } & \text { Slurry Mix Evaporator } \\ \text { SRNL } & \text { Savannah River National Laboratory } \\ \text { SRR } & \text { Savannah River Remediation } \\ \text { TOC } & \text { Total Organic Carbon } \\ \text { TTQAP } & \text { Task Technical and Quality Assurance Plan } \\ \text { TTR } & \text { Technical Task Request } \\ \text { WSE } & \text { Waste Solidification Engineering } \\ \% \text { RD } & \text { Percent Relative Difference } \\ \text { \%RSD } & \text { Percent Relative Standard Deviation }\end{array}$


SRNL-STI-2013-00139

Revision 0

\subsection{Introduction}

The Savannah River National Laboratory (SRNL) has been working with the Savannah River Remediation (SRR) Defense Waste Processing Facility (DWPF) in the development and implementation of the flammability control strategy for DWPF's melter operation during the processing of Sludge Batch 8 (SB8). The flammability control strategy relies on measurements that are performed on Slurry Mix Evaporator (SME) samples by the DWPF Laboratory. One of the SME analytes is nitrate, and the current Technical Safety Requirement (TSR) upper limit for the concentration of nitrate is $30,000 \mathrm{ppm}$ for melter off gas (MOG) flammability.

Due to the potential acid demand of SB8, the limit of 30,000 ppm of nitrate in the SME product could be exceeded in DWPF, which under the current MOG flammability TSR would require remediation of the SME. To avoid the need for this remediation, the nitrate range for the MOG flammability calculation will need to be expanded. A technical task request (TTR) [1] was made by SRR's Waste Solidification Engineering (WSE) organization to SRNL requesting support in completing this expansion and the associated Type I calculation for SB8. This calculation has been completed and documented by Choi [2]. The resulting flammability control strategy relies on analytical measurements of SME samples that are performed by the DWPF Laboratory, and WSE has requested via a TTR [3] that SRNL provide an evaluation of the associated analytical error and error structure for these measurements. SRNL responded to this request by issuing a Task Technical and Quality Assurance Plan (TTQAP) [4] for the activities that are to be conducted in support of the completion of that task.

The sample data supporting the control strategy include measurements of total organic carbon (TOC) and measurements by Ion Chromatography (IC) of select anions: nitrate, oxalate, and formate. Specifically, SRNL is being requested to provide (1) an evaluation of the uncertainties associated with these measurements, (2) an approach for integrating the uncertainties of these measurements into the flammability control strategy, and (3) equations that may be used for facility implementation of the control strategy with uncertainties. JMP Version 9.0.0 was used to support the analyses presented in this report [5].

\subsection{IC and TOC Measurements by the DWPF Laboratory}

Measurements generated by the DWPF Laboratory for standards of the analytes of interest to this study were provided to SRNL by Laboratory Operations (Lab OPS) personnel. These data are presented and their uncertainties investigated in this section. For each analyte of interest, the goal of the investigation was to estimate (1) any potential bias in the measurement of the analyte and (2) the variation in the measurements due to day-to-day effects on the measurement process for that analyte. Both of these uncertainties are of interest since the average of the measurements from a set of SME samples for the given analyte may be biased by the influence of each of these two sources of uncertainty.

Table A1 in Appendix A provides IC and TOC measurements recently generated by the DWPF Laboratory. Included in the IC results are measurements for formate, nitrate, soluble oxalate, and total oxalate for standards at 2 parts per million (ppm) and $16 \mathrm{ppm}$ for each of these anions. Table A1 also contains measurements for TOC standards at concentrations of 1 and $20 \mathrm{ppm}$. TOC measurements are typically collected in pairs: one pair (a measurement of the $1 \mathrm{ppm}$ standard and a measurement of the $20 \mathrm{ppm}$ standard) before and a similar pair after process samples are processed. The before pair is identified as TOC1 measurements and the after pair is labeled as TOC2 in Table A1. 
The dates that the measurements were recorded are also provided as part of the information in Table A1. Also in this table are the differences between the measured and reference values as well as the percent relative differences $(\% \mathrm{RDs})$ :

$\%$ Relative Difference $=\quad 100 \% \times($ Measurement - Reference Value $) /($ Reference Value $)$,

The analyses conducted in this report utilize the values of these $\%$ relative differences.

Exhibit A1 in Appendix A provides variability plots of the DWPF Laboratory \%RDs from Table A1 for the $2 \mathrm{ppm}$ and $16 \mathrm{ppm}$ standards for formate, nitrate, and oxalate (both soluble and total) and for the $1 \mathrm{ppm}$ and $20 \mathrm{ppm}$ standards for TOC. The values are arranged by date. Since some of the values in these plots appear to be potential outliers, a screening process was conducted for these data. Exhibit A2 of Appendix A provides boxplots for the results for each standard for each analyte of interest. These boxplots served as the basis for screening the data for potential outliers. An immediate concern, as one works with a data set, is the potential for the presence of one or more outliers in the data and the possible impact of such outliers on the conclusions being made about the data. Based on the boxplots, those values for an analyte that are beyond the distances given by:

upper quartile $+1.5 *$ (interquartile range) and lower quartile $-1.5 *$ (interquartile range),

(based upon the quartiles computed from the set of values with the interquartile range being the difference between the $1^{\text {st }}$ quartile and $3^{\text {rd }}$ quartile) are considered as questionable points (i.e., potential outliers). These are the points that fall beyond the "whiskers" (the lines extended from the boxes) of the boxplots, and they were excluded from the screened data. In the exhibit, each of the boxplots in the left column provides a display that includes all of the data while the corresponding plot in the right column shows the data after the screening process. For the analyses that follow, each investigation is conducted both with the full set of values and with the set of screened values. The objective of this approach is to select the outcome from the dual investigations that yields the more conservative (i.e., larger) estimated uncertainty.

In Exhibit A3 of Appendix A, the set of \%RDs for each standard for each analyte (e.g., the measurements for the $2 \mathrm{ppm}$ formate standard) is investigated using all of the measurements from Table A1 and using a random effects model to represent two sources of variation: between-day (or day-to-day) effects and within-day effects. Both sources of variation in the measurements of each analyte are of interest. The DWPF Lab measures samples from a given SME batch to determine the concentrations of the analytes of interest in evaluating the SME. Typically 4 measurements are obtained for each analyte with the average of the 4 measurements being computed and used to represent the content of that analyte in the SME. It should be noted that the variation in the 4 results for each analyte reflect not only the contributions to variability from the within-day effects on the measurement process but also sample-to-sample variation associated with the SME sampling process. However, since all four of the measurements are conducted on the same day, they are subject to a single random effect from the between-day or day-to-day source of variation, and this source of variation is be estimated from the measurements of the standards and subsequently accounted for as part of this uncertainty analysis.

Estimating this between-day variation is one of the goals of the analyses presented in Exhibit A3. The estimates of the two variance components of the random effects model for each set of values are provided in the tabulated portion of the exhibit under the heading "REML Variance Components Estimates." Exhibit 1 provides a closer look at the results for the $2 \mathrm{ppm}$ standard for formate. The variance component for "Date" represents the estimated variance for the between-day random effect, 
which has a value of $6.098(\%)^{2}$ in Exhibit 1 . The square root of this value is the \% relative standard deviation (\%RSD) for these measurements with a value of $2.47 \%$. The estimate of the within-day variance (in $\%^{2}$ units) is indicated in the "Residual" line of this table. For the $2 \mathrm{ppm}$ standard for the formate data, this value is $5.892(\%)^{2}$. However, during the flammability control calculations, the estimate of this variance component for the SME samples will be determined directly from the measurements of those samples. The estimate of the between-day variance component for each of the standards for each analyte, which is of interest in this analysis, is presented in Exhibit A3 in the manner illustrated by Exhibit 1.

\begin{tabular}{|lrrrrrr|}
\hline REML Variance Component Estimates & & & & \\
Random Effect & Var Ratio & Var Component & Std Error & 95\% Lower & 95\% Upper & Pct of Total \\
Date & 1.0348594 & 6.0978682 & 1.5359399 & 3.9321362 & 10.719743 & 50.857 \\
Residual & & 5.8924604 & 0.8760424 & 4.4921277 & 8.0710572 & 49.143 \\
Total & 11.990329 & & & & 100.000 \\
& & & & & & \\
\hline
\end{tabular}

Exhibit 1. REML Estimates of Variance Components

The results from the analyses in Exhibit A3 also provide an estimate of the bias associated with the set of \%RDs of the measurements for each of the standards at one reference value for each analyte of interest. Since the \%RDs are being utilized in these analyses, the bias information provided is in the form of a $\%$ relative bias. The estimate of the $\%$ relative bias is indicated in the exhibit as the "Intercept" under the "Parameter Estimates" table. If the p-value given in this table is 0.05 or smaller, the hypothesis of a zero bias is rejected at a significance level of 5\%. Otherwise, the hypothesis cannot be rejected at this significance level. A 95\% confidence interval for the bias is also provided in this table. If this bias is positive and statistically significant, the larger of the two endpoints for the confidence interval is a bound on the potential bias at more than $95 \%$ confidence. If the bias is negative and statistically significant, then the more negative value is a bound, with more than $95 \%$ confidence, on the potential negative bias affecting these measurements.

Exhibit 2 provides a closer look at the results for the $2 \mathrm{ppm}$ standard for formate. The p-value is $<0.0001$, which indicates that the null hypothesis of a zero bias is rejected at the $5 \%$ significance level. The results indicate a positive bias, which may be bounded at a value of $2.997 \%$ with more than $95 \%$ confidence (as indicated in Exhibit 2).

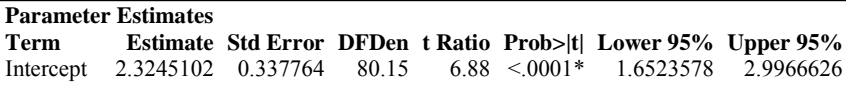

Exhibit 2. Estimate of \% Relative Bias

Table 1 provides a summary of the vital information (i.e., the estimate of the between-day variance component and a bound on the bias, when statistically significant) for each standard and analyte from Exhibit A3. Once again, the analyses presented in Exhibit A3 utilized all of the data from Table A1. 
Table 1. Summary Information from Exhibit A3

Using All of the Measurements from Table A1

\begin{tabular}{|c|c|c|c|c|}
\hline Analyte & $\begin{array}{c}\text { Reference } \\
\text { Value } \\
\mathbf{( p p m )}\end{array}$ & $\begin{array}{c}\text { Estimate of } \\
\text { Day-to-Day } \\
\text { Variance (\%) }\end{array}$ & $\begin{array}{c}\text { Day-to-Day } \\
\text { \%RSD }\end{array}$ & $\begin{array}{c}\text { \% Bias Bound at } \\
\text { Greater than 95\% } \\
\text { Confidence }\end{array}$ \\
\hline Formate & 2 & 6.098 & 2.47 & 3.00 \\
\hline Nitrate & 16 & 2.507 & 1.58 & 1.58 \\
\hline & 2 & 7.430 & 2.73 & No significant bias \\
\hline Soluble Oxalate & 2 & 2.383 & 1.54 & No significant bias \\
\hline & 16 & 9.060 & 3.01 & 4.30 \\
\hline Total Oxalate & 2 & 4.576 & 2.14 & -1.27 \\
\hline & 16 & 21.143 & 4.60 & 5.41 \\
\hline TOC & 1 & 25.500 & 5.05 & No significant bias \\
\hline & 20 & 7.375 & 2.72 & No significant bias \\
\hline & & 4.010 & 2.00 & No significant bias \\
\hline
\end{tabular}

Exhibit A4 in Appendix A provides the same analyses as Exhibit A3 except that only the screened data were used. Table 2 summarizes the vital information from the analyses of the screened data that is presented in Exhibit A4.

Table 2. Summary Information from Exhibit A4

Using the Screened Measurements from Table A1

\begin{tabular}{|c|c|c|c|c|}
\hline Analyte & $\begin{array}{c}\text { Reference } \\
\text { Value } \\
\mathbf{( p p m )}\end{array}$ & $\begin{array}{c}\text { Estimate of } \\
\text { Day-to-Day } \\
\text { Variance } \mathbf{( \%}^{\mathbf{2}} \mathbf{)}\end{array}$ & $\begin{array}{c}\text { Day-to-Day } \\
\text { \%RSD }\end{array}$ & $\begin{array}{c}\text { \% Bias Bound at } \\
\text { Greater than 95\% } \\
\text { Confidence }\end{array}$ \\
\hline Formate & 2 & 2.858 & 1.69 & 3.03 \\
\hline Nitrate & 16 & 0.292 & 0.54 & 1.95 \\
\hline Soluble Oxalate & 2 & 5.946 & 2.44 & No significant bias \\
\hline & 16 & 0.730 & 0.85 & 0.76 \\
\hline Total Oxalate & 2 & 7.092 & 2.66 & 4.53 \\
\hline & 16 & 1.915 & 1.38 & No significant bias \\
\hline TOC & 1 & 16.958 & 4.12 & 5.03 \\
\hline & 20 & 4.686 & 2.16 & No significant bias \\
\hline & & 6.342 & 2.52 & No significant bias \\
\hline
\end{tabular}

For each anion and for TOC, the between-day variance is a contributor to the uncertainty for the measurements of the samples from a given SME batch. This is a batch-to-batch variation affecting the measurements of a SME batch. Accounting for this variation as part of the uncertainty in the measurements of each of the anions and of TOC is addressed in the discussions that follow. In addition, for those analytes with a statistically significant bias, the effects of the biases on the flammability control strategy are to be conservatively accounted for. This is addressed below as part of subsequent discussions presented in this report. 
SRNL-STI-2013-00139

Revision 0

\subsection{The Maximum TOC versus $\mathrm{NO}_{3}$ at $60 \% \mathrm{LFL}$}

As stated in the Introduction, efforts are underway at SRNL to support WSE in the development of a melter flammability control strategy for SB8 [2]. The strategy relies on a SME batch satisfying a constraint on the TOC content of the SME based upon the nitrate content of the SME and tied to an estimate of the amount of antifoam used in the preparation of the SME batch. TOC versus nitrate constraints were developed by Choi [2] for three (3) different antifoam additions: 728, 894, and 1,017 gallons (gal). All three constraints were developed to be applicable for nitrate concentrations in the interval from 10,000 to 70,000 ppm. Before a SME batch is to be transferred to the melter, WSE must establish that the TOC concentration to the nitrate concentration satisfies the restrictions imposed by Choi's analysis [2]. WSE has requested that the efforts of this study address a conservative implementation of these constraints (i.e., one based on a linearized representation of the TOC to nitrate relationship) for flammability control (see Appendix B). Given that even this linearized implementation strategy relies on process measurements, WSE must account for the uncertainties of the measurements to confidently achieve a successful implementation of the flammability control strategy. The uncertainties of these measurements were investigated earlier in this report, and the purpose of this section is to establish the linearized approach, to provide a set of equations that integrate the measurement uncertainties into the flammability control strategy, and to provide sample calculations for these equations to illustrate their impact on the flammability control strategy.

The TOC constraints developed by Choi maintain the contributors to melter flammability to less than $60 \%$ of the lower flammability limit (LFL) [2]. The maximum allowable TOC was determined by Choi as a polynomial function of the nitrate concentration, and a different functional constraint on the TOC values was developed for each of three antifoam additions. Since all of these constraints were developed over the interval of nitrate concentrations from $10,000 \mathrm{ppm}$ to $70,000 \mathrm{ppm}$, that interval of nitrate concentrations is the range of applicability for these functions. If the contents of a SME batch yield an average nitrate measurement outside of the interval from 10,000 to $70,000 \mathrm{ppm}$, then satisfying the TOC constraint may not be a reliable control for addressing melter flammability concerns for that SME batch. In the sections that follow, the impacts of the TOC and nitrate measurement uncertainties (that were determined in the previous section of this report) on the assessment of the nitrate content of the SME and for controlling melter flammability are investigated.

\subsection{Assessing the Nitrate Content of the SME}

To implement the melter flammability control strategy, there is a need to assure with high confidence that the nitrate $\left(\mathrm{NO}_{3}\right)$ content of the SME falls within the interval from 10,000 to 70,000 ppm. Demonstrating that this condition is met for a given SME batch will rely on the four nitrate

measurements from that batch. The average of these measurements may be represented by $\overline{\mathrm{NO}_{3}}$. The standard error, or the 1-sigma standard uncertainty, of this value may be represented by $\mathrm{se}_{\mathrm{NO}_{3}}$, and its value is also computed from the four nitrate values: the standard error of the mean is the standard deviation computed from the four nitrate values divided by 2 . In addition, from the discussion above, two other effects are to be accounted for in meeting this constraint: any bias in the measurements of the nitrate standards and the batch-to-batch 1-sigma relative uncertainty. For the nitrate results, there was a statistically significant bias in the measurements for the $16 \mathrm{ppm}$ standards for the screened data. The mean of the measurements for the $16 \mathrm{ppm}$ standards for the screened data was high relative to the nominal value for that standard, and it is not clear when the bias may become less significant for lower nitrate concentrations. Thus, the assumption being made in regard to nitrate measurements is 
that the measured nitrate content of the SME may be slightly more than the actual amount (on average). Thus, in assessing if the nitrate content falls within the interval from 10,000 to 70,000 ppm, it is conservative to not adjust the nitrate content when it is compared to the upper limit (i.e., the $70,000 \mathrm{ppm}$ limit) but to make the adjustment for a potentially high bias when the nitrate content is compared to the lower limit (i.e., the 10,000 ppm limit).

From the discussion above, a bound on the potentially high bias in the nitrate measurements, with more than $95 \%$ confidence, was determined to be $0.76 \%$ of the nominal $16 \mathrm{ppm}$ value. In assessing the nitrate content of a SME batch against the $70,000 \mathrm{ppm}$ lower limit, the average nitrate measurement will be adjusted for this potential bias, and that adjustment will be made by decreasing the amount of nitrate by $0.76 \%$ (i.e., by multiplying the average nitrate content by 0.9924 ).

In assessing the batch-to-batch variation affecting the nitrate measurements, the largest estimate of the between-day component of variance from Tables 1 and 2 yields a $\%$ RSD of $2.73 \%$ (determined from all of the measurements for the 2 ppm standard).

Following the guidelines and notational conventions presented in [6] (which may differ somewhat from the notation typically used in statistical models), an input term, $\delta_{\mathrm{NO}_{3}}$, representing the batch-tobatch random effect is to be added to the measurement equations for the $\mathrm{NO}_{3}$ content in the SME.

If Low $\mathrm{NO}_{3}$ is used to represent the difference between the estimated $\mathrm{NO}_{3}$ content of the $\mathrm{SME}$ and the lower limit of $10,000 \mathrm{ppm}$, then the measurement equation for the measurand, $\mathrm{Low}_{\mathrm{NO}_{3}}$, may be written as:

Equation 1.

$$
\operatorname{Low}_{\mathrm{NO}_{3}}=0.9924 \cdot\left(\overline{\mathrm{NO}_{3}}+\delta_{\mathrm{NO}_{3}}\right)-10000>0
$$

where $\overline{\mathrm{NO}_{3}}$ represents the average of the $\mathrm{NO}_{3}$ concentration measurements for the 4 samples of the given SME batch. This average has a 1-sigma standard uncertainty of $\mathrm{se}_{\overline{\mathrm{NO}_{3}}}$,

0.9924 is the adjustment for a potentially high bias in the nitrate measurements, and

$\delta_{\mathrm{NO}_{3}}$ represents the batch-to-batch source of variation affecting the nitrate measurements for a SME batch. For the evaluation of Low $\mathrm{NO}_{3}$ by Equation 1, the value of $\delta_{\mathrm{NO}_{3}}$ is zero. Its 1-sigma relative standard uncertainty is the batch-to-batch variation and is represented by $\mathrm{s}_{\mathrm{NO}_{3}}$, and based upon the discussion above, its value is given by $2.73 \%$ of the $\overline{\mathrm{NO}_{3}}$ value.

The guidance provided in [6] relies on the use of a Taylor's series expansion of the measurement equation to approximate the variance of the measurand. The expression of this approximation is simplified if the errors associated with the inputs to the measurement equation are uncorrelated, which is the case for the errors associated with the two input terms of Equation $1^{f}$. For this simple

The errors associated with the terms in the other measurement equations investigated as part of this study are also assumed to be uncorrelated. 
measurement equation, the variance of $\mathrm{Low}_{\mathrm{NO}_{3}}$ is equal to its Taylor's series expansion. However, for conservatism, the bias adjustment term (i.e., 0.9924) will not be included in the estimation of variance. The estimation of the variance of $\mathrm{Low}_{\mathrm{NO}_{3}}$ is facilitated by estimating the standard deviations of $\overline{\mathrm{NO}_{3}}$ and $\delta_{\mathrm{NO}_{3}}$ by $\mathrm{se}_{\mathrm{NO}_{3}}$ and $\mathrm{s}_{\mathrm{NO}_{3}}$, respectively, as indicated in Equation 2:

Equation 2.

$$
\text { Est. } \operatorname{Var}\left(\operatorname{Low}_{\mathrm{NO}_{3}}\right)=\left[\left(\mathrm{se} \frac{\mathrm{NO}_{3}}{{ }^{2}}+\left(\mathrm{s}_{\mathrm{\delta}_{\mathrm{NO}_{3}}}\right)^{2}\right]\right.
$$

where Est.Var $\left(\mathrm{Low}_{\mathrm{NO}_{3}}\right)$ represents the estimated variance of $\mathrm{Low}_{\mathrm{NO}_{3}}$, the estimated difference between the nitrate content of the SME and the lower limit of 10,000 ppm.

If $\mathrm{High}_{\mathrm{NO}_{3}}$ is used to represent the estimated difference between the upper limit of 70,000 ppm and the $\mathrm{NO}_{3}$ content of the SME, then the measurement equation for the measurand, $\mathrm{High}_{\mathrm{NO}_{3}}$, may be written as:

Equation 3.

$$
\operatorname{High}_{\mathrm{NO}_{3}}=70000-\left(\overline{\mathrm{NO}_{3}}+\delta_{\mathrm{NO}_{3}}\right)>0
$$

where $\overline{\mathrm{NO}_{3}}$ represents the average of the $\mathrm{NO}_{3}$ concentration measurements for the 4 samples of the given SME batch. This average has a 1-sigma standard uncertainty of $\mathrm{se}_{\overline{\mathrm{NO}_{3}}}$, and

$\delta_{\mathrm{NO}_{3}}$ represents the batch-to-batch source of variation affecting the nitrate measurements for a SME batch. For the evaluation of $\mathrm{High}_{\mathrm{NO}_{3}}$ by Equation 3, the value of $\delta_{\mathrm{NO}_{3}}$ is zero. Its 1-sigma relative standard uncertainty is represented by $\mathrm{s}_{\mathrm{N}_{\mathrm{NO}_{3}}}$ and based upon the analyses summarized above, it has a value given by $2.73 \%$ of the $\overline{\mathrm{NO}_{3}}$ value.

Once again, for this simple measurement equation, the variance of $\mathrm{High}_{\mathrm{NO}_{3}}$ is equal to its Taylor's series expansion, and the estimation of the variance of $\mathrm{High}_{\mathrm{NO}_{3}}$ is facilitated by estimating the standard deviations of $\overline{\mathrm{NO}_{3}}$ and $\delta_{\mathrm{NO}_{3}}$ by $\mathrm{se}_{\mathrm{NO}_{3}}$ and $\mathrm{s}_{\delta_{\mathrm{NO}_{3}}}$, respectively, as indicated by:

Equation 4.

$$
\text { Est. } \operatorname{Var}\left(\mathrm{High}_{\mathrm{NO}_{3}}\right)=\left(\mathrm{se}_{\mathrm{NO}_{3}}\right)^{2}+\left(\mathrm{s}_{\delta_{\mathrm{NO}_{3}}}\right)^{2}
$$

where Est.Var $\left(\mathrm{High}_{\mathrm{NO}_{3}}\right)$ represents the estimated variance of $\mathrm{High}_{\mathrm{NO}_{3}}$. 
To determine that the nitrate content of the SME is within the interval from $10,000 \mathrm{ppm}$ to 70,000 ppm the inequalities of Equations 1 and 3 must be met after accounting for the uncertainties estimated in Equations 2 and 4. To determine the uncertainty at 95\% confidence, the square roots of the estimates of the variances for Equations 1 and 3 must be multiplied by an appropriate Student's t statistic. Since a two-sided evaluation for the nitrate content is needed, a two-tailed $t$ statistic may be used. For this situation, 3 degrees of freedom will be assumed for the estimated variances of Equations 1 and 3. For 3 degrees of freedom, the upper 2.5\%-tail of the Student's t distribution is 3.182. That is,

Equation 5.

$$
\text { Low }_{\mathrm{NO}_{3}}-\mathrm{t}_{(0.025,3)} \cdot\left(\text { Est. } \operatorname{var}\left(\text { Low }_{\mathrm{NO}_{3}}\right)\right)^{0.5}>0
$$

and

Equation 6.

$$
\mathrm{High}_{\mathrm{NO}_{3}}-\mathrm{t}_{(0.025,3)} \cdot\left(\text { Est. } \operatorname{var}\left(\mathrm{High}_{\mathrm{NO}_{3}}\right)\right)^{0.5}>0
$$

Thus, the nitrate content of the SME is acceptable if

Equation 7.

$$
\left(\overline{\mathrm{NO}_{3}} \cdot 0.9924\right)-10000-3.182 \cdot \sqrt{\left(\mathrm{se}_{\mathrm{NO}_{3}}\right)^{2}+\left(\mathrm{s}_{\delta_{\mathrm{NO}_{3}}}\right)^{2}}>0
$$

and

Equation 8.

$$
70000-\overline{\mathrm{NO}_{3}}-3.182 \cdot \sqrt{\left(\mathrm{se}_{\overline{\mathrm{NO}_{3}}}\right)^{2}+\left(\mathrm{s}_{\delta_{\mathrm{NO}_{3}}}\right)^{2}}>0
$$

To illustrate these calculations, consider the four nitrate measurements (in ppm): 23700, 23604, 23229 , and 23433 . The sample mean of these values, $\overline{\mathrm{NO}_{3}}$, is given by 23491.5 , and the 1 -sigma standard uncertainty of the sample mean is given by sample standard deviation divided by the square root of the sample size, or 206.928/2=103.464. The value of $\mathrm{s}_{\delta_{\mathrm{NO}_{3}}}$ is determined by $0.0273 \cdot 23491.5=641.318$.

The result for Equation 7 is

$$
\begin{aligned}
& \left(\overline{\mathrm{NO}_{3}} \cdot 0.9924\right)-10000-3.182 \cdot \sqrt{\left(\mathrm{se}_{\mathrm{NO}_{3}}\right)^{2}+\left(\mathrm{s}_{\delta_{\mathrm{NO}_{3}}}\right)^{2}} \\
& =23491.5 \cdot 0.9924-10000-3.182 \cdot 649.610 \\
& =11245.9045>0
\end{aligned}
$$

which shows acceptability, and the result for Equation 8 is 
SRNL-STI-2013-00139

Revision 0

$$
\begin{aligned}
70000-\overline{\mathrm{NO}_{3}}-3.182 \cdot \sqrt{\left(\mathrm{se}_{\overline{\mathrm{NO}_{3}}}\right)^{2}+\left(\mathrm{s}_{\left.\delta_{\mathrm{NO}_{3}}\right)^{2}}\right.} & =70000-23491.5-2067.059 \\
& =44441.441 \mathrm{>} 0
\end{aligned}
$$

which also shows acceptability.

\subsection{Controlling Melter Flammability via the TOC to $\mathrm{NO}_{3}$ Relationship}

Each of the three functions (indexed by the level of antifoam addition) developed by Choi [2] relating TOC content to nitrate content may be expressed in the form of a $4^{\text {th }}$ degree polynomial, as given by:

Equation 9.

$$
\mathrm{TOC}_{\mathrm{i}}=\mathrm{a}_{\mathrm{i}}+\mathrm{b}_{\mathrm{i}} \cdot\left(\mathrm{NO}_{3}\right)+\mathrm{c}_{\mathrm{i}} \cdot\left(\mathrm{NO}_{3}\right)^{2}+\mathrm{d}_{\mathrm{i}} \cdot\left(\mathrm{NO}_{3}\right)^{3}+\mathrm{e}_{\mathrm{i}} \cdot\left(\mathrm{NO}_{3}\right)^{4}
$$

where the $\mathrm{TOC}_{\mathrm{i}}$ term on the left-hand side of the equation represents the maximum TOC allowed for a SME batch to maintain the DWPF melter system below the $60 \%$ LFL, while the $\mathrm{NO}_{3}$ term on the right-hand side represents the nitrate content of the subsequent melter feed in ppm. The values of the $a_{i}, b_{i}, c_{i}, d_{i}$, and $e_{i}$ coefficients are given in Table 3 for $i=1,2$, and 3, and each value of the $i$ index corresponds to one of the three additions of antifoam (in gal) for which Choi developed the associated TOC versus $\mathrm{NO}_{3}$ relationship.

Table 3. Coefficients for Equations Relating TOC Content to $\mathrm{NO}_{3}$ Content

\begin{tabular}{|c|c|c|c|c|c|c|}
\hline $\mathrm{i}$ & $\begin{array}{c}\text { Antifoam } \\
\text { Addition (gal) }\end{array}$ & $\mathrm{a}_{\mathrm{i}}$ & $\mathrm{b}_{\mathrm{i}}$ & $\mathrm{c}_{\mathrm{i}}$ & $\mathrm{d}_{\mathrm{i}}$ & $\mathrm{e}_{\mathrm{i}}$ \\
\hline 1 & 728 & 7557.6474 & 0.50526028 & $-9.2573423 \mathrm{E}-06$ & $2.3456397 \mathrm{E}-10$ & $-1.6391746 \mathrm{E}-15$ \\
\hline 2 & 894 & 9422.0849 & 0.07089545 & $9.1468028 \mathrm{E}-06$ & $-6.620362 \mathrm{E}-11$ & $5.7959687 \mathrm{E}-17$ \\
\hline 3 & 1,017 & 10556.47 & -0.2167157 & $2.0961912 \mathrm{E}-05$ & $-2.5179967 \mathrm{E}-10$ & $1.0703826 \mathrm{E}-15$ \\
\hline
\end{tabular}

WSE requested that a linearized version of the $\mathrm{TOC}$ to $\mathrm{NO}_{3}$ relationship be developed (see Appendix B) so that individual SME batches that are compliant with the linearized versions (see Equation 10) will be compliant with the family of Choi equations (given by Equation 9) when blended.

Equation 10.

$$
\mathrm{TOC}_{\mathrm{i}}=\mathrm{f}_{\mathrm{i}}+\mathrm{g}_{\mathrm{i}} \cdot\left(\mathrm{NO}_{3}\right)
$$

where, once again, the term $\mathrm{TOC}_{\mathrm{i}}$ term on the left-hand side of the equation represents the maximum TOC allowed to maintain the system below the $60 \%$ LFL based upon a linearized relationship between TOC and $\mathrm{NO}_{3}$, while the $\mathrm{NO}_{3}$ term on the right-hand side represents the nitrate content of the melter feed in ppm. The values of the $f_{i}$ and $g_{i}$ coefficients are given in Table 4 for $i=1,2$, and 3 , and each value of the $i$ index corresponds to one of the three additions of antifoam (in gal) for which Choi developed an associated TOC versus $\mathrm{NO}_{3}$ relationship. The $\mathrm{f}_{\mathrm{i}}$ and $\mathrm{g}_{\mathrm{i}}$ values were selected to help minimize the TOC margin lost in going to the linearized approach over the $\mathrm{NO}_{3}$ interval of 10,000 to $30,000 \mathrm{ppm}$. 
SRNL-STI-2013-00139

Revision 0

\section{Table 4. Coefficients for the Linear Equations \\ Relating TOC Content to $\mathrm{NO}_{3}$ Content}

\begin{tabular}{|c|c|c|c|}
\hline $\mathrm{i}$ & $\begin{array}{c}\text { Antifoam } \\
\text { Addition (gal) }\end{array}$ & $\mathrm{f}_{\mathrm{i}}$ & $\mathrm{g}_{\mathrm{i}}$ \\
\hline 1 & 728 & 8140 & 0.37 \\
\hline 2 & 894 & 6550 & 0.37 \\
\hline 3 & 1,017 & 5300 & 0.37 \\
\hline
\end{tabular}

Each of the linear approximations indicated in Table 4 was selected so that when the limit on the allowed TOC content is met for the linear equation, that outcome ensures that the limit imposed by the corresponding Choi equation is also being met. See Exhibit A5 in Appendix A for graphical comparisons between the Choi and linear equations which show that each Choi equation allows more TOC than the corresponding linear equation.

In the discussion above, no statistically significant bias was indicated for the TOC measurements; while for the nitrate measurements, there was a statistically significant high bias. Thus, to be conservative, an adjustment is made (in the manner of Equation 2) to the nitrate value in Equation 10 for this bias. The direct utilization of the family of equations given by Equation 10 for melter flammability control yields this acceptability equation for a given SME batch:

Equation 11.

$$
\mathrm{D}_{\mathrm{i}}=\mathrm{f}_{\mathrm{i}}+\mathrm{g}_{\mathrm{i}} \cdot 0.9924 \cdot \overline{\mathrm{NO}_{3}}-\overline{\mathrm{TOC}}-\text { other }_{\mathrm{C}}>0
$$

where $D_{i} \quad$ is the measurand, it represents the difference in ppm between the carbon allowed by Equation 10 and the carbon content of the SME, and its value must be positive,

$f_{i} \& g_{i}$ are the coefficients from Table 4 corresponding to the appropriate bound on the gallons of antifoam utilized in the preparation of the SME batch (indexed by i) as determined following the approach discussed in Section 4 below,

0.9924 is the adjustment for a potential high bias in the nitrate measurements, and

$\overline{\mathrm{NO}_{3}}$ represents the average of the $\mathrm{NO}_{3}$ concentration measurements in ppm for the samples of the given SME batch,

$\overline{\text { TOC }}$ represents the average of the TOC concentration measurements in ppm for the samples of the given SME batch, and

other $_{C}$ represents carbon that is present in the SME in a form that is not measured by the TOC analytical protocol. Note, however, that such carbon was included in the determination of the TOC relationship to nitrate given by Equation 9. Its value is associated with the amount of coal in the SME and is bounded in [2] by $240 \mathrm{ppm}$.

The form of Equation 11 is such that the value of $D_{i}$ must be positive for acceptability. That is, the amount of TOC allowed by the use of Equation 10 must be greater than the TOC content of the given SME for the given level of antifoam addition (indexed by i), and this must be true with high confidence after accounting for the uncertainties in the measurements used to make this determination. 
Following the approach and notational conventions of [6], Equation 11 is modified to make it a more complete measurement equation for the determination of $\mathrm{D}_{\mathrm{i}}$. Also in the discussion above, a batch-tobatch 1-sigma relative uncertainty of $2.73 \%$ was identified as affecting the nitrate measurements. For the TOC measurements, a review of the information in Tables 1 and 2 regarding the day-to-day variance components indicates that a conservative estimate of the batch-to-batch 1-sigma relative uncertainty is provided by $2.72 \%$. If the errors for these sources of variation are represented as $\delta_{\mathrm{NO}_{3}}$ and $\delta_{\mathrm{TOC}}$, respectively, they may be added to Equation 11 to obtain a more complete measurement equation for $D_{i}$ (indexed by the value of the antifoam addition) as follows:

Equation 12.

$$
\mathrm{D}_{\mathrm{i}}=\mathrm{f}_{\mathrm{i}}+\mathrm{g}_{\mathrm{i}} \cdot 0.9924 \cdot\left(\overline{\mathrm{NO}_{3}}+\delta_{\mathrm{NO}_{3}}\right)-\left(\overline{\mathrm{TOC}}+\delta_{\mathrm{TOC}}\right)-\text { other }_{\mathrm{C}}>0
$$

The values of $\delta_{\mathrm{NO}_{3}}$ and $\delta_{\mathrm{TOC}}$ are zero in the determination of the value of $\mathrm{D}_{\mathrm{i}}$, but including these terms in the equation for $D_{i}$ allows for their contributions to the variance of $D_{i}$ to be included in the variance propagation for Equation 12 .

A Taylor's series expansion approach is used to estimate the variance of $\mathrm{D}_{\mathrm{i}}$ [6], and as above, for conservatism the bias adjustment term $(0.9924)$ is not included in the estimation of variance. Assuming that the errors in Equation 12 are uncorrelated and that the value for other ${ }_{C}$ is bounding and thus may be considered to be without error, the Taylor's series expansion approach yields:

Equation 13.

$\operatorname{var}\left(\mathrm{D}_{\mathrm{i}}\right) \approx\left(\frac{\partial \mathrm{D}_{\mathrm{i}}}{\partial \overline{\mathrm{NO}_{3}}}\right)^{2} \cdot\left(\mathrm{se}_{\overline{\mathrm{NO}_{3}}}\right)^{2}+\left(\frac{\partial \mathrm{D}_{\mathrm{i}}}{\partial \delta_{\mathrm{NO}_{3}}}\right)^{2} \cdot\left(\mathrm{s}_{\delta_{\mathrm{NO}_{3}}}\right)^{2}+\left(\frac{\partial \mathrm{D}_{\mathrm{i}}}{\partial \overline{\mathrm{TOC}}}\right)^{2} \cdot\left(\mathrm{se} \frac{}{\mathrm{TOC}}\right)^{2}+\left(\frac{\partial \mathrm{D}_{\mathrm{i}}}{\partial \delta_{\mathrm{TOC}}}\right)^{2} \cdot\left(\mathrm{s}_{\delta_{\mathrm{TOC}}}\right)^{2}$

where $\frac{\partial \mathrm{D}_{\mathrm{i}}}{\partial \bullet}$ represents the partial derivative of $\mathrm{D}_{\mathrm{i}}$ with respect to the variable $(\bullet), \mathrm{se}_{\mathrm{NO}_{3}}$ represents the 1-sigma standard uncertainty of the average nitrate measurement of the SME samples, $\mathrm{s}_{\mathrm{NO}_{3}}$ represents the 1-sigma relative standard uncertainty of the batch-to-batch variation in the nitrate measurements (i.e., $2.73 \%$ of the $\overline{\mathrm{NO}_{3}}$ value), $\mathrm{se}_{\mathrm{TOC}}$ represents the 1-sigma standard uncertainty of the average TOC measurement of the SME samples, and $\mathrm{s}_{\delta_{\mathrm{TOC}}}$ represents the 1-sigma relative standard uncertainty of the batch-to-batch variation in the TOC measurements (i.e., $2.72 \%$ of the $\overline{\mathrm{TOC}}$ value). Once again, note that no error term is introduced in Equation 13 for the other $\mathrm{C}_{\mathrm{C}}$ input of Equation 12.

Using this approach, the variance of $\mathrm{D}_{\mathrm{i}}$ is estimated by:

Equation 14.

$$
\operatorname{var}\left(\mathrm{D}_{\mathrm{i}}\right) \approx\left(\mathrm{g}_{\mathrm{i}}\right)^{2} \cdot\left(\mathrm{se}_{\overline{\mathrm{NO}_{3}}}\right)^{2}+\left(\mathrm{g}_{\mathrm{i}}\right)^{2} \cdot\left(\mathrm{s}_{\delta_{\mathrm{NO}_{3}}}\right)^{2}+\left(\mathrm{se}_{\overline{\mathrm{TOC}}}\right)^{2}+\left(\mathrm{s}_{\delta_{\mathrm{TOC}}}\right)^{2}
$$


Note that since the value of $g_{i}$ is 0.37 for each value of the index $i(i=1,2$, and 3 ), the equation for estimating the variance of $D_{i}$ is the same for each value of $i$ and it may be expressed as Equation 15.

$$
\begin{aligned}
\operatorname{var}\left(\mathrm{D}_{1}\right) & =\operatorname{var}\left(\mathrm{D}_{2}\right)=\operatorname{var}\left(\mathrm{D}_{3}\right) \approx(0.37)^{2} \cdot\left(\mathrm{se}_{\mathrm{NO}_{3}}\right)^{2}+(0.37)^{2} \cdot\left(\mathrm{s}_{\delta_{\mathrm{NO}_{3}}}\right)^{2}+\left(\mathrm{se}_{\mathrm{TOC}}\right)^{2}+\left(\mathrm{s}_{\delta_{\mathrm{TOC}}}\right)^{2} \\
& =0.1368 \cdot\left(\mathrm{se} \frac{\mathrm{NO}_{3}}{{ }^{2}}+0.1369 \cdot\left(\mathrm{s}_{\delta_{\mathrm{NO}_{3}}}\right)^{2}+\left(\mathrm{se} \frac{\overline{\mathrm{TOC}}}{2}+\left(\mathrm{s}_{\delta_{\mathrm{TOC}}}\right)^{2}\right.\right.
\end{aligned}
$$

Using the estimates of the batch-to-batch variations discussed above, Equation 15 may be expressed as:

Equation 16.

$$
\begin{aligned}
& \operatorname{var}\left(\mathrm{D}_{1}\right)=\operatorname{var}\left(\mathrm{D}_{2}\right)=\operatorname{var}\left(\mathrm{D}_{3}\right) \approx 0.1369 \cdot\left(\mathrm{se} \frac{\mathrm{NO}_{3}}{{ }^{2}}+0.1369 \cdot\left(\mathrm{s}_{\delta_{\mathrm{NO}_{3}}}\right)^{2}+\left(\mathrm{se} \frac{\overline{\mathrm{TOC}}}{{ }^{2}}+\left(\mathrm{s}_{\delta_{\mathrm{TOC}}}\right)^{2}\right.\right.
\end{aligned}
$$

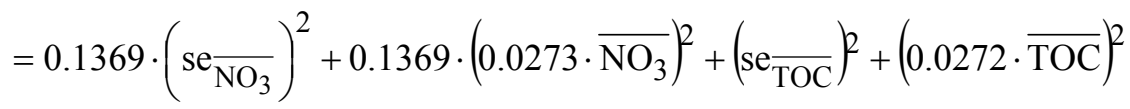

$$
\begin{aligned}
& =0.1369 \cdot\left(\mathrm{se} \frac{\overline{\mathrm{NO}_{3}}}{{ }^{2}}+0.00010203 \cdot\left(\overline{\mathrm{NO}_{3}}\right)^{2}+\left(\mathrm{se}_{\overline{\mathrm{TOC}}}\right)^{2}+0.00073984 \cdot(\overline{\mathrm{TOC}})^{2}\right.
\end{aligned}
$$

\subsection{Meeting the Constraint Imposed by the TOC to Nitrate Relationship}

In meeting the constraint on TOC imposed by its relationship to nitrate, the value for the degrees of freedom for the estimate of the variance of $D_{i}$ is taken to be 3 . This is a conservative approach since it relies on only the degrees of freedom associated with the standard errors of the nitrate and TOC sample means. As indicated in Equation 12, the value of $D_{i}$ must be positive for acceptability. To assure that the value of $\mathrm{D}_{\mathrm{i}}$ is sufficiently positive, it must be larger than its estimated uncertainty at $95 \%$ confidence. To determine the uncertainty at $95 \%$ confidence, the square root of the estimate of the variance of $D_{i}$ must be multiplied by an appropriate Student's t statistic. Since only a one-sided expression of the uncertainty of $\mathrm{D}_{\mathrm{i}}$ is needed, a one-tailed t statistic may be used. For 3 degrees of freedom, the upper 5\%-tail of the Student's t distribution is 2.353 . Thus, the SME batch is acceptable from the perspective of its TOC content if:

Equation 17.

$$
\mathrm{D}_{\mathrm{i}}-\mathrm{t}_{(0.05,3)} \cdot\left(\text { Est. } \operatorname{var}\left(\mathrm{D}_{\mathrm{i}}\right)\right)^{0.5}=\mathrm{D}_{\mathrm{i}}-2.353 \cdot\left(\text { Est. } \operatorname{var}\left(\mathrm{D}_{\mathrm{i}}\right)\right)^{0.5}>0
$$

where $D_{i}$ is determined using Equation 12 (for the associated level of antifoam additions),

$\mathrm{t}_{(0.05,3)}$ is the upper 5\%-tail of the Student's t distribution (i.e., 2.353), and

Est. $\operatorname{var}\left(D_{i}\right)$ represents the estimate of the variance of $D_{i}$, which may be computed using Equation 16 regardless of the antifoam addition being considered. 


\subsection{Example Calculations in Meeting the TOC Constraint}

To illustrate these calculations, they were performed for the nitrate and TOC values shown in Exhibit 3. In this exhibit four values (in ppm) are provided for TOC and nitrate. Also, in this exhibit the averages, standard deviations, and standard errors of the mean are shown. The averages are the simple arithmetic averages of the 4 values for each analyte, and the standard deviations are also computed using the traditional equation for this summary statistic. The standard error of the mean for each analyte is the value of its standard deviation divided by 2 (the square root of the number of observations used to determine the average).

\begin{tabular}{|l|c|c|}
\hline & TOC (ppm) & Nitrate $(\mathrm{ppm})$ \\
& 12500 & 23700 \\
& 12750 & 23604 \\
& 12800 & 23229 \\
& 12666 & 23433 \\
\hline averages & 12679.000 & 23491.500 \\
\hline standard deviations & 131.5193 & 206.9275 \\
\hline standard error of the mean & 65.75966 & 103.46376 \\
\hline
\end{tabular}

\section{Exhibit 3. Illustration of the Calculations for TOC to Nitrate Acceptability}

With these values determined, Equations 11 and 16 can be used to compute the value for $D_{i}$ and its estimated variance, respectively, for each of the antifoam additions (recall that the coefficients $f_{i}$ and $\mathrm{g}_{\mathrm{i}}$ are given in Table 4). Equation 18 provides the value for $\mathrm{D}_{\mathrm{i}}$ (in $\mathrm{ppm}$ ) for each of the antifoam additions.

Equation 18a (728 gal addition of antifoam).

$$
\begin{aligned}
\mathrm{D}_{1} & =\mathrm{f}_{1}+\mathrm{g}_{1} \cdot 0.9924 \cdot \overline{\mathrm{NO}_{3}}-\overline{\mathrm{TOC}}-\text { other }_{\mathrm{C}} \\
& =8140+0.37 \cdot 0.9924 \cdot 23491.500-12679.000-240 \\
& =3846.797 \\
& >0
\end{aligned}
$$

Equation 18b (894 gal addition of antifoam).

$$
\begin{aligned}
\mathrm{D}_{2} & =\mathrm{f}_{2}+\mathrm{g}_{2} \cdot 0.9924 \cdot \overline{\mathrm{NO}_{3}}-\overline{\mathrm{TOC}}-\text { other }_{\mathrm{C}} \\
& =6550+0.37 \cdot 0.9924 \cdot 23491.500-12679.000-240 \\
& =2256.797 \\
& >0
\end{aligned}
$$


Equation $18 \mathrm{c}(1,017$ gal addition of antifoam).

$$
\begin{aligned}
\mathrm{D}_{3} & =\mathrm{f}_{3}+\mathrm{g}_{3} \cdot 0.9924 \cdot \overline{\mathrm{NO}_{3}}-\overline{\mathrm{TOC}}-\text { other }_{\mathrm{C}} \\
& =5300+0.37 \cdot 0.9924 \cdot 23491.500-12679.000-240 \\
& =1006.797 \\
& >0
\end{aligned}
$$

The estimated variance for each of these $\mathrm{D}_{\mathrm{i}}$ values is computed using the same equation (i.e., Equation 16) and the result is provided in Equation 19.

Equation 19.

$$
\begin{aligned}
\operatorname{var}\left(\mathrm{D}_{1}\right) & =\operatorname{var}\left(\mathrm{D}_{2}\right)=\operatorname{var}\left(\mathrm{D}_{3}\right) \approx 0.1369 \cdot\left(\mathrm{se}_{\overline{\mathrm{NO}_{3}}}\right)^{2}+\left(\mathrm{se}_{\overline{\mathrm{TOC}}}\right)^{2}+0.00010203 \cdot\left(\overline{\mathrm{NO}_{3}}\right)^{2}+0.00073984 \cdot(\overline{\mathrm{TOC}})^{2} \\
& =0.1369 \cdot(103.46376)^{2}+(65.75966)^{2}+0.00010203 \cdot(23491.500)^{2}+0.00073984 \cdot(12679.000)^{2} \\
& =181029.62
\end{aligned}
$$

With these calculations in hand, the question of meeting the TOC constraint as defined by Equation 17 may be addressed for each candidate level of antifoam addition. Equation 20 provides the results (expressed in ppm of TOC) from these determinations.

Equation 20a (728 gal addition).

$$
\begin{aligned}
\mathrm{D}_{1}-\mathrm{t}_{(0.05,3)} \cdot\left(\text { Est. } \operatorname{var}\left(\mathrm{D}_{1}\right)\right)^{0.5} & =3846.797-2.353 \cdot(181029.62)^{0.5} \\
& =3846.797-2.353 \cdot 425.476 \\
& =2845.65 \\
& >0
\end{aligned}
$$

Equation 20b (894 gal addition).

$$
\begin{aligned}
\mathrm{D}_{2}-\mathrm{t}_{(0.05,3)} \cdot\left(\text { Est. } \operatorname{var}\left(\mathrm{D}_{2}\right)\right)^{0.5} & =2256.797-2.353 \cdot(181029.62)^{0.5} \\
& =2256.797-2.353 \cdot 425.476 \\
& =1255.65 \\
& >0
\end{aligned}
$$


SRNL-STI-2013-00139

Revision 0

Equation 20c (1,017 gal addition).

$$
\begin{aligned}
\mathrm{D}_{3}-\mathrm{t}_{(0.05,3)} \cdot\left(\text { Est. } \operatorname{var}\left(\mathrm{D}_{3}\right)\right)^{0.5} & =1006.797-2.353 \cdot(181029.62)^{0.5} \\
& =1006.797-2.353 \cdot 425.476 \\
& =5.65 \\
& >0
\end{aligned}
$$

The results from the evaluations of Equation 20 indicate that the measured TOC content for this example is acceptable for antifoam additions of 728,894 , or 1,017 gal.

\subsection{Estimation of the Antifoam Addition}

As described above, the relationship between the maximum allowable TOC content and the $\mathrm{NO}_{3}$ content of a SME batch was associated with the total amount of antifoam used to prepare the SME batch. Three levels for this total antifoam amount in gallons were established by Choi [2]: 728, 894, and 1,017 gal. As part of his study, Choi developed a relationship between the maximum amount of carbon that would be generated from each of these bounding amounts of antifoam and the $\mathrm{NO}_{3}$ content of the SME. These relationships are given by:

Equation 21.

$$
\left(\text { Antifoam }_{\mathrm{Ci}}\right)^{2}=\mathrm{h}_{\mathrm{i}}+\mathrm{j}_{\mathrm{i}} \cdot\left(\mathrm{NO}_{3}\right) \Rightarrow \text { Antifoam }_{\mathrm{C}}=\sqrt{\mathrm{h}_{\mathrm{i}}+\mathrm{j}_{\mathrm{i}} \cdot\left(\mathrm{NO}_{3}\right)}
$$

where Antifoam ${ }_{\mathrm{Ci}}$ represents the maximum amount of carbon in ppm that would be generated from antifoam additions for three cases indexed by i: 1 corresponds to 728 gal, 2 to 894 gal, and finally, 3 to 1,017 gal of antifoam, and $\mathrm{NO}_{3}$ represents the nitrate content of the SME in ppm. The $\mathrm{h}_{\mathrm{i}}$ and $\mathrm{j}_{\mathrm{i}}$ values, which are provided in Table 5, are from the models developed by Choi [2].

Table 5. Coefficients for the Equations Relating Maximum Carbon Content from Antifoam Additions to $\mathrm{NO}_{3}$ Content

\begin{tabular}{|c|c|c|c|}
\hline $\mathrm{i}$ & $\begin{array}{c}\text { Antifoam } \\
\text { Addition (gal) }\end{array}$ & $\mathrm{h}_{\mathrm{i}}$ & $\mathrm{j}_{\mathrm{i}}$ \\
\hline 1 & 728 & 5117745.1 & -35.869438 \\
\hline 2 & 894 & 7884790.5 & -55.545316 \\
\hline 3 & 1,017 & 10373798 & -73.602487 \\
\hline
\end{tabular}

Thus, DWPF's melter flammability control strategy requires that WSE confidently estimate an upper bound on the amount of carbon attributable to the antifoam added during the processing of each SME batch, and then use this result to confidently establish one of the three antifoam levels developed by Choi (i.e., for additions of no more than 728, 894, or 1,017 gal) as the upper bound on the gallons of antifoam added during the processing of the SME batch. The importance of this outcome is that it establishes the appropriate $\mathrm{TOC}$ to $\mathrm{NO}_{3}$ relationship that must be used in satisfying Equation 17 as discussed in Section 3. 


\subsection{Bounding the Total Amount of Antifoam Additions Made to a SME Batch}

Thus, there is a need to estimate the amount of antifoam that was added during DWPF's preparation of a given SME batch. From the previous section, there is a relationship between the nitrate content of the SME and the limit on the carbon generated by antifoam additions to that SME for three different levels of antifoam addition: 728, 894, and 1,017 gal. Thus, one may estimate the amount of antifoam added during the preparation of a SME batch by estimating an upper bound on the carbon content due to antifoam additions for the prepared SME. The estimation is conducted by backing out contributions to the measured TOC concentration in the SME from the oxalate and the formate concentrations that are measured in the SME. The resulting adjusted TOC value provides a basis for estimating the amount of carbon in the SME attributable to antifoam. When this estimate is bounded at $95 \%$ confidence by accounting for its uncertainty, the resulting bounded amount of carbon attributable to antifoam must be below the carbon allowed by the level of antifoam (i.e., one of the three values:728, 894 , or 1,017 gal) that is selected to be appropriate for the given SME batch.

As the measured TOC concentration in the SME is adjusted for carbon contributions from oxalate and formate, these adjustments are to be modified in a conservative manner to handle the potential bias in the formate and in the oxalate results (as suggested by the analysis of the standards, the results from which were summarized in Tables 1 and 2). From the results of the formate standards, there was a consistently high bias for both the 2 and 16 ppm standards. Using the largest upper bound for this bias from Tables 1 and 2, a bound on this relative bias, with more than 95\% confidence, is provided by $3.03 \%$. The formate measurements will be adjusted for this potential bias, and that adjustment will be made by reducing the amount of carbon contributed by formate by $3.03 \%$ (i.e., by multiplying the average formate content by 0.9697 ).

In representing the oxalate content of the SME, either the soluble or total oxalate concentration measurements in ppm may be used. The analytical procedures used to measure soluble oxalate are more quickly completed than those used to measure the total oxalate. If the difference between the allowable amount of antifoam and the estimated amount is positive using the soluble oxalate value (after uncertainties have been accounted for), then the SME batch is acceptable in regards to its estimated amount of added antifoam. For the acceptability decision to be conservative, regardless of whether the soluble or total oxalate is used, the values bounding the bias and representing the batchto-batch variability must cover the results for both forms of oxalate. From Tables 1 and 2, a conservative approach to bound the bias is to use the value of $5.41 \%$ (provided in the results from the 2 ppm standard for total oxalate in the Table 1, which utilized all of the measurements). The oxalate measurements will be adjusted for this potential bias, and that adjustment will be made by reducing the amount of carbon contributed by oxalate by $5.41 \%$ (i.e., by multiplying the average oxalate content by 0.9459 ). For the batch-to-batch variation, the conservative value of $5.05 \%$ (provided in the results from Table 1 for all of the measurements of the $16 \mathrm{ppm}$ standard) is to be used. Once again, with this conservative approach, either soluble or total oxalate may be used to evaluate the acceptability of a SME batch in meeting the constraint imposed by Equation 21 .

Note that to add conservatism to the implementation of Equation 21, the nitrate value in the equation will not be adjusted for the potential high bias in the nitrate measurements. Based upon this approach, the restriction imposed on the contents of the SME by Equation 21 may be expressed as:

Equation 22.

$$
\mathrm{M}_{\mathrm{Ci}}=\sqrt{\mathrm{h}_{\mathrm{i}}+\mathrm{j}_{\mathrm{i}} \cdot \overline{\mathrm{NO}_{3}}}-\overline{\mathrm{TOC}}+\mathrm{f}_{\mathrm{C}} \cdot \overline{\text { formate }} \cdot 0.9697+\mathrm{o}_{\mathrm{C}} \cdot \overline{\text { oxalate }} \cdot 0.9459>0
$$


where

$\mathrm{M}_{\mathrm{Ci}} \quad$ is the measurand; it represents the difference between the allowable concentration in ppm of carbon from antifoam at a level indexed by $i$ (where $i=1$ represents 728 gal, 2 represents 894 gal, and 3 represents 1,017 gal) and the estimated amount of carbon attributable to antifoam; and the difference must be positive,

$\mathrm{i} \quad$ is used as an index for the level of antifoam addition in gallons, $\mathrm{i}=1$ (728 gal), 2 (894 gal), and 3 (1,017 gal).

$h_{i} \& j_{i}$ are the coefficients corresponding to the $i^{\text {th }}$ level of antifoam (their values are provided in Table 5),

$\overline{\mathrm{NO}_{3}}$ represents (as above) the average of the $\mathrm{NO}_{3}$ concentration measurements in ppm for the samples of the given SME batch,

$\overline{\mathrm{TOC}}$ is (as above) the average of the TOC measurements in ppm for the samples from the given SME batch,

$\overline{\text { formate }}$ is the average of the formate measurements in ppm for the samples from the SME batch $^{f}$,

$\overline{\text { oxalate }}$ is the average of the oxalate measurements in ppm for the samples from the SME batch,

0.9697 is included in the measurement equation to adjust (with a better than $95 \%$ confidence) for the potential bias in the formate measurement,

$\mathrm{f}_{\mathrm{C}}$ is the conversion factor needed to determine the carbon contributed by the formate content of the SME in ppm,

$\mathrm{o}_{\mathrm{C}}$ is the conversion factor needed to determine the carbon contributed by the oxalate content of the SME in ppm, and

0.9459 is included in the measurement equation to adjust (with a better than $95 \%$ confidence) for the potential bias in the oxalate measurement,

The conversion factor for formate is $0.26681 \mathrm{ppm}$ carbon per ppm of formate as determined by:

Equation 23.

$\frac{1 \mathrm{mg} \text { formate }}{\mathrm{kg} \mathrm{SME} \mathrm{Slurry}} \times \frac{1 \mathrm{gram}}{1000 \mathrm{mg}} \times \frac{1 \text { gmole formate }}{45.0177 \mathrm{~g} \text { formate }} \times \frac{1 \text { gmole carbon }}{1 \text { gmole formate }} \times \frac{12.011 \mathrm{~g} \text { carbon }}{1 \text { gmole carbon }} \times \frac{1000 \mathrm{mg}}{1 \text { gram }}=0.266806 \frac{1 \mathrm{mg} \text { carbon }}{\mathrm{kg} \text { SME Slurry }}$

and for oxalate, the factor is $0.27292 \mathrm{ppm}$ carbon per ppm of oxalate as determined by

\footnotetext{
${ }^{f}$ Note the multiplications by 0.9697 and 0.9459 . This makes the adjustments for the potential biases in the measured formate and oxalate content, respectively, of the SME.
} 
Equation 24.

$\frac{1 \mathrm{mg} \text { oxalate }}{\text { kg SME Slurry }} \times \frac{1 \text { gram }}{1000 \mathrm{mg}} \times \frac{1 \text { gmole oxalate }}{88.019 \text { g oxalate }} \times \frac{2 \text { gmole carbon }}{1 \text { gmole oxalate }} \times \frac{12.011 \mathrm{~g} \text { carbon }}{1 \text { gmole carbon }} \times \frac{1000 \mathrm{mg}}{1 \text { gram }}=0.27292 \frac{1 \mathrm{mg} \text { carbon }}{\mathrm{kg} \mathrm{SME} \mathrm{Slurry}}$

The uncertainty in meeting the constraint imposed by Equation 22 is now determined. To facilitate this process, the equation is modified using notational conventions suggested by [6] to develop a more complete measurement equation. This involves introducing terms for the batch-to-batch effects for $\mathrm{NO}_{3}, \delta_{\mathrm{NO}_{3}}$, for formate, $\delta_{\text {formate }}$, for oxalate, $\delta_{\text {oxalate }}$, and for TOC, $\delta_{\mathrm{TOC}}$. The measurement equation resulting from these additions is given by:

Equation 25.

$$
\begin{aligned}
& \mathrm{M}_{\mathrm{Ci}}=\sqrt{\mathrm{h}_{\mathrm{i}}+\mathrm{j}_{\mathrm{i}} \cdot\left(\overline{\mathrm{NO}_{3}}+\delta_{\mathrm{NO}_{3}}\right)-\left(\overline{\mathrm{TOC}}+\delta_{\mathrm{TOC}}\right)} \\
& +\mathrm{f}_{\mathrm{C}} \cdot\left(\overline{\text { formate }}+\delta_{\text {formate }}\right) \cdot 0.9697+\mathrm{o}_{\mathrm{C}} \cdot\left(\overline{\text { oxalate }}+\delta_{\text {oxalate }}\right) \cdot 0.9459>0
\end{aligned}
$$

where all of the terms in Equation 25 are as indicated above with the addition of the $\delta$ terms representing the batch-to-batch variations in the measurements of these analytes.

For the evaluation of Equation 25, the values of $\delta_{\mathrm{NO}_{3}}, \delta_{\mathrm{TOC}}, \delta_{\text {formate }}$, and $\delta_{\text {oxalate }}$ are taken to be zero. The variance of $\mathrm{M}_{\mathrm{Ci}}$, the difference between the allowable carbon from antifoam and the estimated carbon from antifoam, will be estimated using a Taylor's series expansion approach as utilized above, and as above, for conservatism the bias adjustment terms (0.9697 and 0.9459) are not included in the estimation of variance. The resulting estimated variance is provided in the following equation:

Equation 26.

$$
\begin{aligned}
\operatorname{var}\left(\mathrm{M}_{\mathrm{Ci}}\right) \approx & \left(\frac{\partial \mathrm{M}_{\mathrm{Ci}}}{\partial \overline{\mathrm{NO}_{3}}}\right)^{2} \cdot\left(\mathrm{se}_{\overline{\mathrm{NO}_{3}}}\right)^{2}+\left(\frac{\partial \mathrm{M}_{\mathrm{Ci}}}{\partial \delta_{\mathrm{NO}_{3}}}\right)^{2} \cdot\left(\mathrm{s}_{\delta_{\mathrm{NO}_{3}}}\right)^{2} \\
& +\left(\frac{\partial \mathrm{M}_{\mathrm{Ci}}}{\partial \overline{\mathrm{TOC}}}\right)^{2} \cdot\left(\operatorname{se} \frac{\overline{\mathrm{TOC}}}{2}+\left(\frac{\partial \mathrm{M}_{\mathrm{Ci}}}{\partial \delta_{\mathrm{TOC}}}\right)^{2} \cdot\left(\mathrm{s}_{\delta_{\mathrm{TOC}}}\right)^{2}\right. \\
& +\left(\frac{\partial \mathrm{M}_{\mathrm{Ci}}}{\partial \overline{\text { formate }}}\right)^{2} \cdot\left(\mathrm{se}_{\text {formate }}\right)^{2}+\left(\frac{\partial \mathrm{M}_{\mathrm{Ci}}}{\partial \delta_{\text {formate }}}\right)^{2} \cdot\left(\mathrm{s}_{\delta_{\text {formate }}}\right)^{2} \\
& +\left(\frac{\partial \mathrm{M}_{\mathrm{Ci}}}{\partial \overline{\text { oxalate }_{\text {oxale }}}}\right)^{2} \cdot\left(\mathrm{se}_{\overline{\text { oxalate }}}\right)^{2}+\left(\frac{\partial \mathrm{M}_{\mathrm{Ci}}}{\partial \delta_{\text {oxalate }}}\right)^{2} \cdot\left(\mathrm{s}_{\delta_{\text {oxalate }}}\right)^{2}
\end{aligned}
$$


where $\operatorname{var}\left(\mathrm{M}_{\mathrm{Ci}}\right)$ represents the estimate of the variance of the difference between the allowable carbon content from the $i^{\text {th }}(i=1,2$, or 3$)$ level of antifoam addition and the estimated carbon content from the addition; $\frac{\partial \mathrm{M}_{\mathrm{Ci}}}{\partial \bullet}$ represents the partial derivative of $\mathrm{M}_{\mathrm{Ci}}$ with respect to the variable $(\bullet), \mathrm{se}_{\mathrm{NO}_{3}}$ represents the 1-sigma standard uncertainty of the average nitrate measurement of the SME samples, $\mathrm{s}_{\delta_{\mathrm{NO}_{3}}}$ represents the 1-sigma relative standard uncertainty of the batch-to-batch variation in the nitrate measurements (i.e., $2.73 \%$ of the $\overline{\mathrm{NO}_{3}}$ value), se $\frac{}{\mathrm{TOC}}$ represents the 1-sigma standard uncertainty of the average TOC measurement of the SME samples, $\mathrm{s}_{\delta_{\mathrm{TOC}}}$ represents the 1-sigma relative standard uncertainty of the batch-to-batch variation in the TOC measurements (i.e., $2.72 \%$ of the $\overline{\mathrm{TOC}}$ value), $\mathrm{se}_{\overline{\text { formate }}}$ represents the 1-sigma standard uncertainty of the average formate measurement of the SME samples, $\mathrm{s}_{\delta_{\text {formate }}}$ represents the 1-sigma standard uncertainty of the batch-to-batch variation in the formate measurements (based upon the results in Table 1 that value is given by $2.47 \%$ of the formate value), se $\overline{\text { oxalate }}$ represents the 1-sigma standard uncertainty of the average oxalate measurement of the SME samples, where all oxalate values are above detection, and $\mathrm{s}_{\delta_{\text {oxalate }}}$ represents the 1-sigma standard uncertainty of the batch-tobatch variation in the oxalate measurements (based upon the results in Table 1, that value is given by $3.01 \%$ of the oxalate value for the soluble oxalate and $5.05 \%$ of the oxalate value for the total oxalate). To be conservative in allowing either soluble or total oxalate to be used in this evaluation, the larger value, i.e., 5.05\%, is utilized for the 1-sigma standard uncertainty of the batch-to-batch variation for oxalate.

Taking the partial derivatives and noting that the values of $\delta_{\mathrm{NO}_{3}}, \delta_{\mathrm{TOC}}, \delta_{\text {formate }}$, and $\delta_{\text {oxalate }}$ are taken to be zero lead to the following equation:

Equation 27.

$$
\begin{aligned}
& \operatorname{var}\left(\mathrm{M}_{\mathrm{Ci}}\right) \approx\left(0.5 \cdot \mathrm{j}_{\mathrm{i}} \cdot\left(\mathrm{h}_{\mathrm{i}}+\mathrm{j}_{\mathrm{i}} \cdot \overline{\mathrm{NO}_{3}}\right)^{-0.5}\right)^{2} \cdot\left(\mathrm{se} \overline{\mathrm{NO}_{3}}\right)^{2}+\left(0.5 \cdot \mathrm{j}_{\mathrm{i}} \cdot\left(\mathrm{h}_{\mathrm{i}}+\mathrm{j}_{\mathrm{i}} \cdot \overline{\mathrm{NO}_{3}}\right)^{-0.5}\right)^{2} \cdot\left(\mathrm{s}_{\delta_{\mathrm{NO}_{3}}}\right)^{2} \\
& +(-1)^{2} \cdot\left(\mathrm{se}_{\mathrm{TOC}}\right)^{2}+(-1)^{2} \cdot\left(\mathrm{s}_{\delta_{\mathrm{TOC}}}\right)^{2} \\
& +\left(\mathrm{f}_{\mathrm{C}}\right)^{2} \cdot\left(\mathrm{se}_{\overline{\text { formate }}}\right)^{2}+\left(\mathrm{f}_{\mathrm{C}}\right)^{2} \cdot\left(\mathrm{s}_{\delta_{\text {formate }}}\right)^{2} \\
& +\left(\mathrm{o}_{\mathrm{C}}\right)^{2} \cdot\left(\mathrm{se} \frac{}{\text { oxalate }}\right)^{2}+\left(\mathrm{o}_{\mathrm{C}}\right)^{2} \cdot\left(\mathrm{s}_{\delta_{\text {oxalate }}}\right)^{2}
\end{aligned}
$$

Substituting the values for the $\mathrm{s}_{\delta}$ terms and for the $\mathrm{f}_{\mathrm{C}}$ and $\mathrm{o}_{\mathrm{C}}$ terms yields: 
SRNL-STI-2013-00139

Revision 0

Equation 28.

$$
\begin{aligned}
\operatorname{var}\left(\mathrm{M}_{\mathrm{Ci}}\right) \approx & \left(0.5 \cdot \mathrm{j}_{\mathrm{i}} \cdot\left(\mathrm{h}_{\mathrm{i}}+\mathrm{j}_{\mathrm{i}} \cdot \overline{\mathrm{NO}_{3}}\right)^{-0.5}\right)^{2} \cdot\left(\mathrm{se} \overline{\mathrm{NO}_{3}}\right)^{2}+\left(0.5 \cdot \mathrm{j}_{\mathrm{i}} \cdot\left(\mathrm{h}_{\mathrm{i}}+\mathrm{j}_{\mathrm{i}} \cdot \overline{\mathrm{NO}_{3}}\right)^{-0.5}\right)^{2} \cdot\left(0.0273 \cdot \overline{\mathrm{NO}_{3}}\right)^{2} \\
& +(-1)^{2} \cdot(\mathrm{se} \overline{\mathrm{TOC}})^{2}+(-1)^{2} \cdot(0.0272 \cdot \overline{\mathrm{TOC}})^{2} \\
& +(0.26681)^{2} \cdot\left(\mathrm{se}_{\text {formate }}\right)^{2}+(0.26681)^{2} \cdot(0.0247 \cdot \overline{\text { formate }})^{2} \\
& +(0.27292)^{2} \cdot\left(\mathrm{se}_{\overline{\text { oxalate }}}\right)^{2}+(0.27292)^{2} \cdot(0.0505 \cdot \overline{\text { oxalate }})^{2}
\end{aligned}
$$

This equation may be further simplified, for each level of antifoam addition (indexed by i) to

Equation 29.

$$
\begin{aligned}
& \operatorname{var}\left(\mathrm{M}_{\mathrm{Ci}}\right) \approx 0.25 \cdot\left(\mathrm{j}_{\mathrm{i}} \cdot\left(\mathrm{h}_{\mathrm{i}}+\mathrm{j}_{\mathrm{i}} \cdot \overline{\mathrm{NO}_{3}}\right)^{0.5}\right)^{2} \cdot\left(\mathrm{se}_{\overline{\mathrm{NO}_{3}}}\right)^{2}+0.000186323 \cdot\left(\mathrm{j}_{\mathrm{i}} \cdot\left(\mathrm{h}_{\mathrm{i}}+\mathrm{j}_{\mathrm{i}} \cdot \overline{\mathrm{NO}_{3}}\right)^{-0.5}\right)^{2} \cdot\left(\overline{\mathrm{NO}_{3}}\right)^{2} \\
&+\left(\mathrm{se} \frac{\overline{\mathrm{TOC}}}{{ }^{2}}+0.00073984 \cdot(\overline{\mathrm{TOC}})^{2}+0.0711876 \cdot(\mathrm{se} \overline{\text { formate }})^{2}+0.000043431 \cdot(\overline{\text { formate }})^{2}\right. \\
&+0.0744853 \cdot\left(\mathrm{se}_{\overline{\text { oxalate }}}\right)^{2}+0.000189956 \cdot(\overline{\text { oxalate }})^{2}
\end{aligned}
$$

\subsection{Meeting the Constraint on the Antifoam Addition}

The expanded uncertainty of the estimated difference, $\mathrm{M}_{\mathrm{Ci}}$, at $95 \%$ confidence is determined by multiplying the square root of the estimated variance of $\mathrm{M}_{\mathrm{Ci}}$ by an appropriate Student's t statistic. In this case a one-sided confidence statement is needed; so, an upper 5\%-tail of the Student's $t$ distribution will be used. Again, utilizing a conservative 3 degrees of freedom for the estimated variance, the $t$ value is 2.353 . Thus, at $95 \%$ confidence the expanded uncertainty of the difference is 2.353 times the square root of the estimated variance of $\mathrm{M}_{\mathrm{Ci}}$. Thus, for the antifoam content of the SME to be acceptable (at 95\% confidence), the following constraint must be met:

Equation 30.

$$
\mathrm{M}_{\mathrm{Ci}}-2.353 \cdot\left(\operatorname{Var}\left(\mathrm{M}_{\mathrm{Ci}}\right)\right)^{0.5}>0
$$

where, for each level of antifoam indexed by $\mathrm{i}, \mathrm{M}_{\mathrm{Ci}}$ is determined from Equation 22 above, and $\operatorname{Var}\left(\mathrm{M}_{\mathrm{Ci}}\right)$ is the estimate of the variance of $\mathrm{M}_{\mathrm{Ci}}$ determined using Equation 29. The smallest of the three levels of antifoam that meets the constraint imposed by Equation 30 is used to select the appropriate TOC constraint that must also be met (as described in Section 3) for an acceptable SME decision. 


\subsection{Example Calculations in Meeting the Constraint on the Antifoam Addition}

Sample calculations are given for the antifoam acceptability determination for the situation provided in Exhibit 4. In this exhibit four values (in ppm) are provided for TOC, formate, nitrate, and oxalate. Also, the averages, standard deviations, and standard errors of the mean are shown in this exhibit. The averages are the simple arithmetic averages of the 4 values for each analyte, and the standard deviations are also computed using the traditional equation for this summary statistic. The standard error of the mean for each analyte is the value of its standard deviation divided by 2 (the square root of the number of observations used to determine the average).

\begin{tabular}{|c|c|c|c|c|}
\hline & TOC (ppm) & Formate (ppm) & Nitrate (ppm) & Oxalate (ppm) \\
\hline & 12500 & 42144 & 23700 & 4000 \\
\hline & 12750 & 42827 & 23604 & 4150 \\
\hline & 12800 & 41246 & 23229 & 3990 \\
\hline & 12666 & 42783 & 23433 & 4125 \\
\hline averages & 12679.000 & 42250.000 & 23491.500 & 4066.250 \\
\hline standard deviations & 131.5193 & 738.5278 & 206.9275 & 83.0035 \\
\hline standard error of the mean & 65.75966 & 369.26391 & 103.46376 & 41.50176 \\
\hline
\end{tabular}

Exhibit 4. Inputs for the Illustration of Calculations for Antifoam Addition

With these values determined, Equations 22 and 29 can be used to compute the value for $\mathrm{M}_{\mathrm{Ci}}$ and its estimated variance, respectively, for each of the antifoam additions (recall that the coefficients $h_{i}$ and $\mathrm{j}_{\mathrm{i}}$ are given in Table 5). Equation 31 provides the value for $\mathrm{M}_{\mathrm{Ci}}$ for each of the antifoam additions.

Equation 31a (728 gal addition).

$$
\begin{aligned}
\mathrm{M}_{\mathrm{C} 1} & =\sqrt{\mathrm{h}_{1}+\mathrm{j}_{1} \cdot \overline{\mathrm{NO}_{3}}}-\overline{\mathrm{TOC}}+\mathrm{f}_{\mathrm{C}} \cdot \overline{\text { formate }} \cdot 0.9697+\mathrm{o}_{\mathrm{C}} \cdot \overline{\text { oxalate }} \cdot 0.9459 \\
& =\sqrt{5117745.1-35.869438 \cdot 23491.500}-12679.000+0.26681 \cdot 42250.000 \cdot 0.9697 \\
& +0.27292 \cdot 4066.250 \cdot 0.9459 \\
& =1369.5178
\end{aligned}
$$

Equation $31 \mathrm{~b}$ (894 gal addition).

$$
\begin{aligned}
\mathrm{M}_{\mathrm{C} 2} & =\sqrt{\mathrm{h}_{2}+\mathrm{j}_{2} \cdot \overline{\mathrm{NO}_{3}}}-\overline{\mathrm{TOC}}+\mathrm{f}_{\mathrm{C}} \cdot \overline{\text { formate }} \cdot 0.9697+\mathrm{o}_{\mathrm{C}} \cdot \overline{\text { oxalate }} \cdot 0.9459 \\
& =\sqrt{7884790.5-55.545316 \cdot 23491.500}-12679.000+0.26681 \cdot 42250.000 \cdot 0.9697 \\
& +0.27292 \cdot 4066.250 \cdot 0.9459 \\
& =1867.0228
\end{aligned}
$$


Equation 31c (1,017 gal addition).

$$
\begin{aligned}
\mathrm{M}_{\mathrm{C} 3} & =\sqrt{\mathrm{h}_{3}+\mathrm{j}_{3} \cdot \overline{\mathrm{NO}_{3}}}-\overline{\mathrm{TOC}}+\mathrm{f}_{\mathrm{C}} \cdot \overline{\text { formate }} \cdot 0.9697+\mathrm{o}_{\mathrm{C}} \cdot \overline{\text { oxalate }} \cdot 0.9459 \\
& =\sqrt{10373798-73.602487 \cdot 23491.500}-12679.000+0.26681 \cdot 42250.000 \cdot 0.9697 \\
& +0.27292 \cdot 4066.250 \cdot 0.9459 \\
& =2242.0800
\end{aligned}
$$

Equation 32 provides the estimated variance for the $\mathrm{M}_{\mathrm{Ci}}$ value for each antifoam addition.

Equation 32a (728 gal addition).

$$
\begin{aligned}
\operatorname{var}\left(\mathrm{M}_{\mathrm{C} 1}\right) \approx & 0.25 \cdot\left(\mathrm{j}_{1} \cdot\left(\mathrm{h}_{1}+\mathrm{j}_{1} \cdot \overline{\mathrm{NO}_{3}}\right)^{-0.5}\right)^{2} \cdot\left(\mathrm{se} \overline{\mathrm{NO}_{3}}\right)^{2} \\
& +0.000186323 \cdot\left(\mathrm{j}_{1} \cdot\left(\mathrm{h}_{1}+\mathrm{j}_{1} \cdot \overline{\mathrm{NO}_{3}}\right)^{-0.5}\right)^{2} \cdot\left(\overline{\mathrm{NO}_{3}}\right)^{2} \\
& +\left(\mathrm{se} \frac{\mathrm{TOC}}{{ }^{2}}+0.00073984 \cdot(\overline{\mathrm{TOC}})^{2}+0.0711876 \cdot(\mathrm{se} \overline{\text { formate }})^{2}+0.000043431 \cdot(\overline{\text { formate }})^{2}\right. \\
& +0.0744853 \cdot(\mathrm{se} \overline{\text { oxalate }})^{2}+0.000189956 \cdot(\overline{\text { oxalate }})^{2} \\
& =0.25 \cdot\left(-35.869438 \cdot(5117745.1-35.869438 \cdot 23491.500)^{-0.5}\right)^{2} \cdot(103.46376)^{2} \\
& +0.000186323 \cdot\left(-35.869438 \cdot(5117745.1-35.869438 \cdot 23491.500)^{-0.5}\right)^{2} \cdot(23491.500)^{2} \\
& +(65.75966)^{2}+0.00073984 \cdot(12679.000)^{2}+0.0711876 \cdot(369.26391)^{2}+0.000043431 \cdot(42250.000)^{2} \\
& +0.0744853 \cdot(41.50176)^{2}+0.000189956 \cdot(4066.250)^{2} \\
& 213793.566
\end{aligned}
$$


Equation $32 b$ (894 gal addition).

$$
\begin{aligned}
\operatorname{var}\left(\mathrm{M}_{\mathrm{C} 2}\right) \approx & 0.25 \cdot\left(\mathrm{j}_{2} \cdot\left(\mathrm{h}_{2}+\mathrm{j}_{2} \cdot \overline{\mathrm{NO}_{3}}\right)^{-0.5}\right)^{2} \cdot\left(\mathrm{se} \overline{\mathrm{NO}_{3}}\right)^{2} \\
& +0.000186323 \cdot\left(\mathrm{j}_{2} \cdot\left(\mathrm{h}_{2}+\mathrm{j}_{2} \cdot \overline{\mathrm{NO}_{3}}-0.5\right)^{2} \cdot\left(\overline{\mathrm{NO}_{3}}\right)^{2}\right. \\
& +(\mathrm{se} \overline{\mathrm{TOC}})^{2}+0.00073984 \cdot(\overline{\mathrm{TOC}})^{2}+0.0711876 \cdot\left(\mathrm{se} \frac{\overline{\text { formate }}}{{ }^{2}+0.000043431 \cdot(\overline{\text { formate }})^{2}}\right. \\
& +0.0744853 \cdot(\mathrm{se} \overline{\text { oxalate }})^{2}+0.000189956 \cdot(\overline{\mathrm{oxalate}})^{2} \\
& =0.25 \cdot\left(-55.545316 \cdot(7884790.5-55.545316 \cdot 23491.500)^{-0.5}\right)^{2} \cdot(103.46376)^{2} \\
& +0.000186323 \cdot\left(-55.545316 \cdot(7884790.5-55.545316 \cdot 23491.500)^{-0.5}\right)^{2} \cdot(23491.500)^{2} \\
& +(65.75966)^{2}+0.00073984 \cdot(12679.000)^{2}+0.0711876 \cdot(369.26391)^{2}+0.000043431 \cdot(42250.000)^{2} \\
& +0.0744853 \cdot(41.50176)^{2}+0.000189956 \cdot(4066.250)^{2} \\
& 213811.284
\end{aligned}
$$


Equation 32c (1,017 gal addition).

$$
\begin{aligned}
\operatorname{var}\left(\mathrm{M}_{\mathrm{C} 2}\right) \approx & 0.25 \cdot\left(\mathrm{j}_{3} \cdot\left(\mathrm{h}_{3}+\mathrm{j}_{3} \cdot \overline{\mathrm{NO}_{3}}\right)^{-0.5}\right)^{2} \cdot\left(\mathrm{se} \overline{\mathrm{NO}_{3}}\right)^{2} \\
& +0.000186323 \cdot\left(\mathrm{j}_{3} \cdot\left(\mathrm{h}_{3}+\mathrm{j}_{3} \cdot \overline{\mathrm{NO}_{3}}\right)^{-0.5}\right)^{2} \cdot\left(\overline{\mathrm{NO}_{3}}\right)^{2} \\
& +\left(\mathrm{se} \frac{\mathrm{TOC}}{{ }^{2}}+0.00073984 \cdot(\overline{\mathrm{TOC}})^{2}+0.0711876 \cdot(\mathrm{se} \overline{\text { formate }})^{2}+0.000043431 \cdot(\overline{\text { formate }})^{2}\right. \\
& +0.0744853 \cdot(\mathrm{se} \overline{\text { oxalate }})^{2}+0.000189956 \cdot(\overline{\mathrm{oxalate}})^{2} \\
& =0.25 \cdot\left(-73.602487 \cdot(10373798-73.602487 \cdot 23491.500)^{-0.5}\right)^{2} \cdot(103.46376)^{2} \\
& +0.000186323 \cdot\left(-73.602487 \cdot(10373798-73.602487 \cdot 23491.500)^{-0.5}\right)^{2} \cdot(23491.500)^{2} \\
& +(65.75966)^{2}+0.00073984 \cdot(12679.000)^{2}+0.0711876 \cdot(369.26391)^{2}+0.000043431 \cdot(42250.000)^{2} \\
& +0.0744853 \cdot(41.50176)^{2}+0.000189956 \cdot(4066.250)^{2} \\
& 213827.928
\end{aligned}
$$

With these calculations in hand, the question of meeting the antifoam constraint as defined by Equation 30 may be addressed for each candidate level of antifoam addition. Equation 33 provides the results from these determinations.

Equation 33a (728 gal addition).

$$
\begin{aligned}
\mathrm{M}_{\mathrm{C} 1}-2.353 \cdot\left(\operatorname{Var}\left(\mathrm{M}_{\mathrm{C} 1}\right)\right)^{0.5} & =1369.5178-2.353 \cdot(213793.566)^{0.5} \\
& =1369.5178-2.353 \cdot 462.378 \\
& =1369.5178-1087.975 \\
& =281.54 \\
& >0
\end{aligned}
$$


SRNL-STI-2013-00139

Revision 0

Equation 33b (894 gal addition).

$$
\begin{aligned}
\mathrm{M}_{\mathrm{C} 2}-2.353 \cdot\left(\operatorname{Var}\left(\mathrm{M}_{\mathrm{C} 2}\right)\right)^{0.5} & =1867.0228-2.353 \cdot(213811.284)^{0.5} \\
& =1867.0228-2.353 \cdot 462.397 \\
& =1867.0228-1088.020 \\
& =779.00 \\
& >0
\end{aligned}
$$

Equation 33c (1,017 gal addition).

$$
\begin{aligned}
\mathrm{M}_{\mathrm{C} 3}-2.353 \cdot\left(\operatorname{Var}\left(\mathrm{M}_{\mathrm{C} 3}\right)\right)^{0.5} & =2242.0800-2.353 \cdot(213827.928)^{0.5} \\
& =2242.0800-2.353 \cdot 462.415 \\
& =2242.0800-1088.062 \\
& =1154.02 \\
& >0
\end{aligned}
$$

Note that the unit of measure for each of the Equation 33 results is ppm of carbon and since all of the results are positive, they indicate that the amount of carbon attributed to antifoam versus the amount allowed is acceptable for each of the antifoam additions considered in this example. Thus, the constraint imposed by TOC to $\mathrm{NO}_{3}$ relationship for the smallest level of antifoam addition (i.e., 728 gal) would be the appropriate constraint to be used in evaluating the acceptability of the given SME batch.

\subsection{Summary}

Measurements of nitrate, oxalate, formate, and TOC standards generated by the DWPF Laboratory are presented in this report and an evaluation of the uncertainties of these measurements is provided. The impact of the uncertainties of these measurements on DWPF's strategy for controlling melter flammability is evaluated. The strategy includes monitoring each SME batch for its nitrate content and its TOC content relative to the nitrate content and relative to the antifoam additions made during the preparation of the SME batch.

A linearized approach for monitoring the relationship between TOC and nitrate is developed, equations are provided that integrate the measurement uncertainties into the flammability control strategy, and sample calculations for these equations are shown to illustrate the impact of the uncertainties on the flammability control strategy. 


\section{References}

[1] Holtzscheiter, E.W. and J.P. Windham, “Technical Task Request: DWPF Melter Off-Gas Flammability Calculation for Sludge Batch 8," HLW-DWPF-TTR-2013-0009, December 4, 2012.

[2] Choi, A.S., "DWPF Melter Off-Gas Flammability Assessment (Sludge Batch 8)," Revision 8, X-CLC-S-00164, March 2013.

[3] Fellinger, T.L., "Technical Task Request: SB8 - Analytical Error Analysis for Melter Off Gas Flammability,” HLW-DWPF-TTR-2013-0022, February 01, 2013.

[4] Edwards, T.B., "Task Technical and Quality Assurance Plan: SB8 - Analytical Error Analysis for Melter Off Gas Flammability," SRNL-RP-2013-00090, Revision 0, February 11, 2013.

[5] JMP Version 9.0.0, SAS Institute, Inc., Cary NC, 1989-2010.

[6] International Organization for Standardization (ISO), Guide to the Expression of Uncertainty in Measurement, ISO, Geneva, 1993, Corrected and reprinted, 1995. 
SRNL-STI-2013-00139

Revision 0

\section{Appendix A.}

Measurement Data for Standards and Statistical Exhibits 
SRNL-STI-2013-00139

Revision 0

Table A1. DWPF Laboratory Measurements of Anion and TOC Standards

\begin{tabular}{|c|c|c|c|c|c|}
\hline Analyte & Date & \begin{tabular}{|c|}
$\begin{array}{c}\text { Measurement } \\
(\mathrm{ppm})\end{array}$ \\
\end{tabular} & $\begin{array}{c}\text { Reference } \\
\text { Value (ppm) }\end{array}$ & diff & $\begin{array}{c}\text { \% rel } \\
\text { diff }\end{array}$ \\
\hline Total Oxalate & $11 / 01 / 2012$ & 2.05 & 2 & 0.05 & 2.5 \\
\hline Total Oxalate & $11 / 03 / 2012$ & 2.12 & 2 & 0.12 & 6 \\
\hline Total Oxalate & $11 / 05 / 2012$ & 2.08 & 2 & 0.08 & 4 \\
\hline Total Oxalate & $11 / 07 / 2012$ & 2.01 & 2 & 0.01 & 0.5 \\
\hline Total Oxalate & $11 / 08 / 2012$ & 2.04 & 2 & 0.04 & 2 \\
\hline Total Oxalate & $11 / 09 / 2012$ & 2.03 & 2 & 0.03 & 1.5 \\
\hline Total Oxalate & $11 / 10 / 2012$ & 2.04 & 2 & 0.04 & 2 \\
\hline Total Oxalate & $11 / 13 / 2012$ & 2.1 & 2 & 0.1 & 5 \\
\hline Total Oxalate & $11 / 14 / 2012$ & 2.1 & 2 & 0.1 & 5 \\
\hline Total Oxalate & $11 / 15 / 2012$ & 2.08 & 2 & 0.08 & 4 \\
\hline Total Oxalate & $11 / 16 / 2012$ & 2.09 & 2 & 0.09 & 4.5 \\
\hline Total Oxalate & $11 / 16 / 2012$ & 2.09 & 2 & 0.09 & 4.5 \\
\hline Total Oxalate & $11 / 17 / 2012$ & 2.1 & 2 & 0.1 & 5 \\
\hline Total Oxalate & $11 / 18 / 2012$ & 2.09 & 2 & 0.09 & 4.5 \\
\hline Total Oxalate & $11 / 19 / 2012$ & 2.06 & 2 & 0.06 & 3 \\
\hline Total Oxalate & $11 / 20 / 2012$ & 2.06 & 2 & 0.06 & 3 \\
\hline Total Oxalate & $11 / 21 / 2012$ & 2.07 & 2 & 0.07 & 3.5 \\
\hline Total Oxalate & $11 / 21 / 2012$ & 2.09 & 2 & 0.09 & 4.5 \\
\hline Total Oxalate & $11 / 22 / 2012$ & 2.09 & 2 & 0.09 & 4.5 \\
\hline Total Oxalate & $11 / 24 / 2012$ & 2.07 & 2 & 0.07 & 3.5 \\
\hline Total Oxalate & $11 / 24 / 2012$ & 2.1 & 2 & 0.1 & 5 \\
\hline Total Oxalate & $11 / 24 / 2012$ & 2.13 & 2 & 0.13 & 6.5 \\
\hline Total Oxalate & $11 / 25 / 2012$ & 2.09 & 2 & 0.09 & 4.5 \\
\hline Total Oxalate & $11 / 26 / 2012$ & 2.09 & 2 & 0.09 & 4.5 \\
\hline Total Oxalate & $11 / 26 / 2012$ & 2.09 & 2 & 0.09 & 4.5 \\
\hline Total Oxalate & $11 / 27 / 2012$ & 2.1 & 2 & 0.1 & 5 \\
\hline Total Oxalate & $11 / 28 / 2012$ & 2.06 & 2 & 0.06 & 3 \\
\hline Total Oxalate & $11 / 28 / 2012$ & 2.11 & 2 & 0.11 & 5.5 \\
\hline Total Oxalate & $11 / 28 / 2012$ & 2.11 & 2 & 0.11 & 5.5 \\
\hline Total Oxalate & $11 / 29 / 2012$ & 2.07 & 2 & 0.07 & 3.5 \\
\hline Total Oxalate & $11 / 30 / 2012$ & 2.09 & 2 & 0.09 & 4.5 \\
\hline Total Oxalate & $12 / 02 / 2012$ & 2.1 & 2 & 0.1 & 5 \\
\hline Total Oxalate & $12 / 02 / 2012$ & 2.15 & 2 & 0.15 & 7.5 \\
\hline Total Oxalate & $12 / 03 / 2012$ & 2.13 & 2 & 0.13 & 6.5 \\
\hline Total Oxalate & $12 / 03 / 2012$ & 2.14 & 2 & 0.14 & 7 \\
\hline Total Oxalate & $12 / 04 / 2012$ & 2.09 & 2 & 0.09 & 4.5 \\
\hline Total Oxalate & $12 / 06 / 2012$ & 2.09 & 2 & 0.09 & 4.5 \\
\hline Total Oxalate & $12 / 06 / 2012$ & 2.16 & 2 & 0.16 & 8 \\
\hline Total Oxalate & $12 / 07 / 2012$ & 2.13 & 2 & 0.13 & 6.5 \\
\hline Total Oxalate & $12 / 08 / 2012$ & 2.08 & 2 & 0.08 & 4 \\
\hline Total Oxalate & $12 / 09 / 2012$ & 2.07 & 2 & 0.07 & 3.5 \\
\hline Total Oxalate & $12 / 10 / 2012$ & 2.14 & 2 & 0.14 & 7 \\
\hline Total Oxalate & $12 / 11 / 2012$ & 2.07 & 2 & 0.07 & 3.5 \\
\hline Total Oxalate & $12 / 12 / 2012$ & 2.08 & 2 & 0.08 & 4 \\
\hline Total Oxalate & $12 / 12 / 2012$ & 2.11 & 2 & 0.11 & 5.5 \\
\hline Total Oxalate & $12 / 13 / 2012$ & 2.06 & 2 & 0.06 & 3 \\
\hline Total Oxalate & $12 / 13 / 2012$ & 2.07 & 2 & \begin{tabular}{|l|l}
0.07 \\
\end{tabular} & 3.5 \\
\hline Total Oxalate & $12 / 14 / 2012$ & 1.93 & 2 & -0.07 & -3.5 \\
\hline Total Oxalate & $12 / 14 / 2012$ & 1.94 & 2 & -0.06 & -3 \\
\hline Total Oxalate & $12 / 14 / 2012$ & 1.97 & 2 & -0.03 & -1.5 \\
\hline Total Oxalate & $12 / 15 / 2012$ & 1.95 & 2 & -0.05 & -2.5 \\
\hline Total Oxalate & $12 / 15 / 2012$ & 1.95 & 2 & -0.05 & -2.5 \\
\hline Total Oxalate & $12 / 15 / 2012$ & 1.97 & 2 & -0.03 & -1.5 \\
\hline Total Oxalate & $12 / 16 / 2012$ & 1.98 & 2 & -0.02 & -1 \\
\hline Total Oxalate & $12 / 17 / 2012$ & 1.99 & 2 & -0.01 & -0.5 \\
\hline Total Oxalate & $12 / 20 / 2012$ & 2.04 & 2 & 0.04 & 2 \\
\hline Total Oxalate & $12 / 20 / 2012$ & 2.05 & 2 & 0.05 & 2.5 \\
\hline Total Oxalate & $12 / 21 / 2012$ & 2.01 & 2 & 0.01 & 0.5 \\
\hline Total Oxalate & $12 / 21 / 2012$ & 2.05 & 2 & 0.05 & 2.5 \\
\hline Total Oxalate & $12 / 23 / 2012$ & 1.84 & 2 & -0.16 & -8 \\
\hline Total Oxalate & $12 / 23 / 2012$ & 1.96 & 2 & -0.04 & -2 \\
\hline Total Oxalate & $12 / 23 / 2012$ & 2.03 & 2 & 0.03 & 1.5 \\
\hline Total Oxalate & $12 / 23 / 2012$ & 2.03 & 2 & 0.03 & 1.5 \\
\hline Total Oxalate & $12 / 24 / 2012$ & 1.89 & 2 & -0.11 & -5.5 \\
\hline Total Oxalate & $12 / 25 / 2012$ & 1.91 & 2 & -0.09 & -4.5 \\
\hline Total Oxalate & $12 / 27 / 2012$ & 2.01 & 2 & \begin{tabular}{|l|}
0.01 \\
\end{tabular} & 0.5 \\
\hline Total Oxalate & $12 / 28 / 2012$ & 2.02 & 2 & 0.02 & 1 \\
\hline Total Oxalate & $12 / 29 / 2012$ & 2.02 & 2 & 0.02 & 1 \\
\hline Total Oxalate & $12 / 30 / 2012$ & 2.02 & 2 & 0.02 & 1 \\
\hline Total Oxalate & $12 / 31 / 2012$ & 2.01 & 2 & 0.01 & 0.5 \\
\hline Total Oxalate & $12 / 31 / 2012$ & 2.02 & 2 & 0.02 & 1 \\
\hline Total Oxalate & $01 / 01 / 2013$ & 2.02 & 2 & 0.02 & 1 \\
\hline Total Oxalate & $01 / 01 / 2013$ & 2.02 & 2 & 0.02 & 1 \\
\hline Total Oxalate & $01 / 02 / 2013$ & 2.02 & 2 & 0.02 & 1 \\
\hline Total Oxalate & $01 / 03 / 2013$ & 1.98 & 2 & -0.02 & -1 \\
\hline Total Oxalate & $01 / 03 / 2013$ & 2 & 2 & 0 & 0 \\
\hline Total Oxalate & $01 / 04 / 2013$ & 2.01 & 2 & 0.01 & 0.5 \\
\hline Total Oxalate & $01 / 05 / 2013$ & 1.98 & 2 & -0.02 & -1 \\
\hline Total Oxalate & $01 / 05 / 2013$ & 1.99 & 2 & -0.01 & -0.5 \\
\hline Total Oxalate & $01 / 06 / 2013$ & 1.99 & 2 & -0.01 & -0.5 \\
\hline Total Oxalate & $01 / 07 / 2013$ & 1.99 & 2 & -0.01 & -0.5 \\
\hline Total Oxalate & 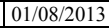 & 2.15 & 2 & 0.15 & 7.5 \\
\hline Total Oxalate & $01 / 08 / 2013$ & 2.21 & 2 & 0.21 & 10.5 \\
\hline
\end{tabular}

\begin{tabular}{|c|c|c|c|c|c|}
\hline Analyte & Date & \begin{tabular}{|c|}
$\begin{array}{c}\text { Measurement } \\
\text { (ppm) }\end{array}$ \\
\end{tabular} & $\begin{array}{c}\text { Reference } \\
\text { Value (ppm) }\end{array}$ & diff & $\begin{array}{l}\text { \% rel } \\
\text { diff }\end{array}$ \\
\hline Total Oxalate & $01 / 09 / 2013$ & \begin{tabular}{r|}
2.21 \\
\end{tabular} & 2 & 0.21 & 10.5 \\
\hline Total Oxalate & $01 / 10 / 2013$ & 2.23 & 2 & 0.23 & 11.5 \\
\hline Total Oxalate & $01 / 11 / 2013$ & 2.11 & 2 & 0.11 & 5.5 \\
\hline Total Oxalate & $01 / 11 / 2013$ & 2.18 & 2 & 0.18 & 9 \\
\hline Total Oxalate & $01 / 12 / 2013$ & 2.09 & 2 & 0.09 & 4.5 \\
\hline Total Oxalate & $01 / 13 / 2013$ & 2.15 & 2 & 0.15 & 7.5 \\
\hline Total Oxalate & $01 / 13 / 2013$ & 2.15 & 2 & 0.15 & 7.5 \\
\hline Total Oxalate & $01 / 13 / 2013$ & 2.15 & 2 & 0.15 & 7.5 \\
\hline Total Oxalate & $01 / 14 / 2013$ & 2.15 & 2 & 0.15 & 7.5 \\
\hline Total Oxalate & $01 / 14 / 2013$ & 2.18 & 2 & 0.18 & 9 \\
\hline Total Oxalate & $01 / 14 / 2013$ & 2.19 & 2 & 0.19 & 9.5 \\
\hline Total Oxalate & $01 / 14 / 2013$ & 2.24 & 2 & 0.24 & 12 \\
\hline Total Oxalate & $01 / 15 / 2013$ & 2.28 & 2 & 0.28 & 14 \\
\hline Total Oxalate & $01 / 16 / 2013$ & 2.27 & 2 & 0.27 & 13.5 \\
\hline Total Oxalate & $01 / 16 / 2013$ & 2.4 & 2 & 0.4 & 20 \\
\hline Total Oxalate & $01 / 17 / 2013$ & 2.26 & 2 & 0.26 & 13 \\
\hline Total Oxalate & $01 / 17 / 2013$ & 2.28 & 2 & 0.28 & 14 \\
\hline Total Oxalate & $01 / 18 / 2013$ & 2.4 & 2 & 0.4 & 20 \\
\hline Total Oxalate & $01 / 19 / 2013$ & 2.21 & 2 & 0.21 & 10.5 \\
\hline Total Oxalate & $01 / 19 / 2013$ & 2.3 & 2 & 0.3 & 15 \\
\hline Total Oxalate & $01 / 20 / 2013$ & 2.28 & 2 & 0.28 & 14 \\
\hline Total Oxalate & $01 / 20 / 2013$ & 2.31 & 2 & 0.31 & 15.5 \\
\hline Total Oxalate & $01 / 21 / 2013$ & 2.25 & 2 & 0.25 & 12.5 \\
\hline Total Oxalate & $01 / 21 / 2013$ & 2.32 & 2 & 0.32 & 16 \\
\hline Total Oxalate & $01 / 22 / 2013$ & 2.3 & 2 & 0.3 & 15 \\
\hline Total Oxalate & $01 / 22 / 2013$ & 2.3 & 2 & 0.3 & 15 \\
\hline Total Oxalate & $01 / 23 / 2013$ & 2.27 & 2 & 0.27 & 13.5 \\
\hline Total Oxalate & $01 / 23 / 2013$ & 2.36 & 2 & 0.36 & 18 \\
\hline Total Oxalate & $01 / 24 / 2013$ & 2.27 & 2 & 0.27 & 13.5 \\
\hline Total Oxalate & $01 / 24 / 2013$ & 2.29 & 2 & 0.29 & 14.5 \\
\hline Total Oxalate & $01 / 25 / 2013$ & 2.06 & 2 & 0.06 & 3 \\
\hline Total Oxalate & $01 / 25 / 2013$ & 2.06 & 2 & 0.06 & 3 \\
\hline Total Oxalate & \begin{tabular}{|l|l|}
$01 / 26 / 2013$ \\
\end{tabular} & 2 & 2 & 0 & 0 \\
\hline Total Oxalate & $01 / 26 / 2013$ & 2 & 2 & 0 & 0 \\
\hline Total Oxalate & $01 / 26 / 2013$ & 2 & 2 & 0 & 0 \\
\hline Total Oxalate & $01 / 26 / 2013$ & 2.01 & 2 & 0.01 & 0.5 \\
\hline Total Oxalate & $01 / 26 / 2013$ & 2.01 & 2 & 0.01 & 0.5 \\
\hline Total Oxalate & $01 / 27 / 2013$ & 2.01 & 2 & 0.01 & 0.5 \\
\hline Total Oxalate & $01 / 28 / 2013$ & 2.01 & 2 & 0.01 & 0.5 \\
\hline Total Oxalate & $01 / 28 / 2013$ & 2.01 & 2 & 0.01 & 0.5 \\
\hline Total Oxalate & $01 / 28 / 2013$ & 2.01 & 2 & 0.01 & 0.5 \\
\hline Total Oxalate & $01 / 29 / 2013$ & 2.08 & 2 & 0.08 & 4 \\
\hline Total Oxalate & $01 / 30 / 2013$ & 2.06 & 2 & 0.06 & 3 \\
\hline Total Oxalate & $01 / 31 / 2013$ & 2.01 & 2 & 0.01 & 0.5 \\
\hline Total Oxalate & $02 / 01 / 2013$ & 2.01 & 2 & 0.01 & 0.5 \\
\hline Total Oxalate & $02 / 01 / 2013$ & 2.02 & 2 & 0.02 & 1 \\
\hline Total Oxalate & \begin{tabular}{|l|l|l|}
$02 / 013$ \\
\end{tabular} & 2.02 & 2 & 0.02 & 1 \\
\hline Total Oxalate & 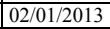 & 2.03 & 2 & 0.03 & 1.5 \\
\hline Total Oxalate & $02 / 02 / 2013$ & 2.05 & 2 & 0.05 & 2.5 \\
\hline Total Oxalate & $02 / 03 / 2013$ & 2.06 & 2 & 0.06 & 3 \\
\hline Total Oxalate & $02 / 04 / 2013$ & 2.06 & 2 & 0.06 & 3 \\
\hline Total Oxalate & $02 / 04 / 2013$ & 2.08 & 2 & 0.08 & 4 \\
\hline Total Oxalate & $02 / 05 / 2013$ & 2.06 & 2 & 0.06 & 3 \\
\hline Total Oxalate & $02 / 05 / 2013$ & 2.09 & 2 & 0.09 & 4.5 \\
\hline Total Oxalate & $02 / 06 / 2013$ & 2.12 & 2 & 0.12 & 6 \\
\hline Total Oxalate & $02 / 07 / 2013$ & 2.09 & 2 & \begin{tabular}{|l|}
0.09 \\
\end{tabular} & 4.5 \\
\hline Total Oxalate & $02 / 07 / 2013$ & 2.1 & 2 & $\begin{array}{ll}0.1 \\
\end{array}$ & 5 \\
\hline Total Oxalate & $02 / 08 / 2013$ & 2.02 & 2 & 0.02 & 1 \\
\hline Total Oxalate & $02 / 09 / 2013$ & 1.94 & 2 & \begin{tabular}{|l|}
-0.06 \\
\end{tabular} & -3 \\
\hline Total Oxalate & \begin{tabular}{|l|l|l}
$02 / 09 / 2013$ \\
\end{tabular} & 2.01 & 2 & 0.01 & 0.5 \\
\hline Total Oxalate & $02 / 09 / 2013$ & 2.15 & 2 & 0.15 & 7.5 \\
\hline Total Oxalate & $02 / 10 / 2013$ & 2.07 & 2 & 0.07 & 3.5 \\
\hline Total Oxalate & $11 / 01 / 2012$ & 15.88 & 16 & \begin{tabular}{|l|}
-0.12 \\
\end{tabular} & -0.75 \\
\hline Total Oxalate & $11 / 03 / 2012$ & 15.89 & 16 & \begin{tabular}{|l|}
-0.11 \\
\end{tabular} & \begin{tabular}{|l|}
-0.6875 \\
\end{tabular} \\
\hline Total Oxalate & $11 / 05 / 2012$ & 15.96 & 16 & \begin{tabular}{|l|}
-0.04 \\
\end{tabular} & $\begin{array}{l}-0.25 \\
\end{array}$ \\
\hline Total Oxalate & \begin{tabular}{|l|l|}
$11 / 07 / 2012$ \\
\end{tabular} & 15.67 & 16 & \begin{tabular}{|l|}
-0.33 \\
\end{tabular} & -2.0625 \\
\hline Total Oxalate & $11 / 08 / 2012$ & 15.79 & 16 & \begin{tabular}{|l|}
-0.21 \\
\end{tabular} & -1.3125 \\
\hline Total Oxalate & $11 / 09 / 2012$ & 15.77 & 16 & -0.23 & -1.4375 \\
\hline Total Oxalate & $11 / 10 / 2012$ & 15.77 & 16 & \begin{tabular}{|l|}
-0.23 \\
\end{tabular} & \begin{tabular}{|l|}
-1.4375 \\
\end{tabular} \\
\hline Total Oxalate & $11 / 13 / 2012$ & 15.81 & 16 & \begin{tabular}{|l|}
-0.19 \\
\end{tabular} & \begin{tabular}{|l|l|}
-1.1875 \\
\end{tabular} \\
\hline Total Oxalate & $11 / 14 / 2012$ & 15.85 & 16 & -0.15 & \begin{tabular}{|l|}
-0.9375 \\
\end{tabular} \\
\hline Total Oxalate & $11 / 15 / 2012$ & 15.77 & 16 & \begin{tabular}{|l|}
-0.23 \\
\end{tabular} & \begin{tabular}{|l}
-1.4375 \\
\end{tabular} \\
\hline Total Oxalate & \begin{tabular}{|l|l|}
$11 / 16 / 2012$ \\
\end{tabular} & 15.85 & 16 & \begin{tabular}{|l|}
-0.15 \\
\end{tabular} & \begin{tabular}{|l}
-0.9375 \\
\end{tabular} \\
\hline Total Oxalate & $11 / 16 / 2012$ & 16.32 & 16 & \begin{tabular}{|l|}
0.32 \\
\end{tabular} & 2 \\
\hline Total Oxalate & $11 / 17 / 2012$ & 16.33 & 16 & \begin{tabular}{|l|}
0.33 \\
\end{tabular} & 2.0625 \\
\hline Total Oxalate & $11 / 18 / 2012$ & 15.92 & 16 & \begin{tabular}{|l|}
-0.08 \\
\end{tabular} & -0.5 \\
\hline Total Oxalate & $11 / 19 / 2012$ & 15.78 & 16 & \begin{tabular}{|l|}
-0.22 \\
\end{tabular} & -1.375 \\
\hline Total Oxalate & $11 / 20 / 2012$ & 16.44 & 16 & \begin{tabular}{|l|}
0.44 \\
\end{tabular} & 2.75 \\
\hline Total Oxalate & $11 / 21 / 2012$ & 15.55 & 16 & \begin{tabular}{|l|}
-0.45 \\
\end{tabular} & \begin{tabular}{|l|}
-2.8125 \\
\end{tabular} \\
\hline Total Oxalate & $11 / 21 / 2012$ & 15.87 & 16 & -0.13 & -0.8125 \\
\hline Total Oxalate & $11 / 22 / 2012$ & 15.89 & 16 & \begin{tabular}{|l|}
-0.11 \\
\end{tabular} & \begin{tabular}{|l}
-0.6875 \\
\end{tabular} \\
\hline Total Oxalate & $11 / 24 / 2012$ & 16.06 & 16 & \begin{tabular}{|l|}
0.06 \\
\end{tabular} & \begin{tabular}{|l}
0.375 \\
\end{tabular} \\
\hline Total Oxalate & $11 / 24 / 2012$ & 16.1 & 16 & \begin{tabular}{|l|}
0.1 \\
\end{tabular} & 0.625 \\
\hline
\end{tabular}


SRNL-STI-2013-00139

Revision 0

Table A1. DWPF Laboratory Measurements of Anion and TOC Standards

\begin{tabular}{|c|c|c|c|c|c|}
\hline Analyte & Date & \begin{tabular}{|c|}
$\begin{array}{c}\text { Measurement } \\
\text { (ppm) }\end{array}$ \\
\end{tabular} & $\begin{array}{c}\text { Reference } \\
\text { Value (ppm) }\end{array}$ & diff & $\begin{array}{l}\text { \% rel } \\
\text { diff }\end{array}$ \\
\hline Total Oxalate & $11 / 24 / 2012$ & $\begin{array}{r}16.51 \\
\end{array}$ & 16 & 0.51 & 3.1875 \\
\hline Total Oxalate & $11 / 25 / 2012$ & 16.45 & 16 & 0.45 & 2.8125 \\
\hline Total Oxalate & $11 / 26 / 2012$ & 15.54 & 16 & -0.46 & -2.875 \\
\hline Total Oxalate & $11 / 26 / 2012$ & 15.86 & 16 & -0.14 & -0.875 \\
\hline Total Oxalate & $11 / 27 / 2012$ & 16.05 & 16 & 0.05 & 0.3125 \\
\hline Total Oxalate & $11 / 28 / 2012$ & 15.8 & 16 & -0.2 & -1.25 \\
\hline Total Oxalate & $11 / 28 / 2012$ & 16.05 & 16 & 0.05 & 0.3125 \\
\hline Total Oxalate & $11 / 28 / 2012$ & 16.05 & 16 & 0.05 & 0.3125 \\
\hline Total Oxalate & $11 / 29 / 2012$ & 16.06 & 16 & 0.06 & 0.375 \\
\hline Total Oxalate & $11 / 30 / 2012$ & 15.4 & 16 & -0.6 & -3.75 \\
\hline Total Oxalate & $12 / 02 / 2012$ & 15.98 & 16 & -0.02 & -0.125 \\
\hline Total Oxalate & $12 / 02 / 2012$ & 16.07 & 16 & \begin{tabular}{|l|}
0.07 \\
\end{tabular} & 0.4375 \\
\hline Total Oxalate & $12 / 03 / 2012$ & 16.14 & 16 & 0.14 & 0.875 \\
\hline Total Oxalate & $12 / 03 / 2012$ & 16.75 & 16 & 0.75 & 4.6875 \\
\hline Total Oxalate & $12 / 04 / 2012$ & 15.69 & 16 & -0.31 & -1.9375 \\
\hline Total Oxalate & $12 / 06 / 2012$ & 16.36 & 16 & 0.36 & 2.25 \\
\hline Total Oxalate & $12 / 06 / 2012$ & 16.41 & 16 & 0.41 & 2.5625 \\
\hline Total Oxalate & $12 / 07 / 2012$ & 16.05 & 16 & 0.05 & 0.3125 \\
\hline Total Oxalate & $12 / 08 / 2012$ & 15.82 & 16 & -0.18 & -1.125 \\
\hline Total Oxalate & $12 / 09 / 2012$ & 15.97 & 16 & -0.03 & -0.1875 \\
\hline Total Oxalate & $12 / 10 / 2012$ & 15.85 & 16 & -0.15 & -0.9375 \\
\hline Total Oxalate & $12 / 11 / 2012$ & 16 & 16 & 0 & 0 \\
\hline Total Oxalate & $12 / 12 / 2012$ & 15.91 & 16 & -0.09 & -0.5625 \\
\hline Total Oxalate & $12 / 12 / 2012$ & 16 & 16 & 0 & 0 \\
\hline Total Oxalate & $12 / 13 / 2012$ & 15.97 & 16 & -0.03 & -0.1875 \\
\hline Total Oxalate & $12 / 13 / 2012$ & 16.02 & 16 & 0.02 & 0.125 \\
\hline Total Oxalate & $12 / 14 / 2012$ & 15.73 & 16 & -0.27 & -1.6875 \\
\hline Total Oxalate & $12 / 14 / 2012$ & 15.94 & 16 & -0.06 & -0.375 \\
\hline Total Oxalate & $12 / 14 / 2012$ & 15.97 & 16 & -0.03 & -0.1875 \\
\hline Total Oxalate & $12 / 15 / 2012$ & 16 & 16 & 0 & 0 \\
\hline Total Oxalate & $12 / 15 / 2012$ & 16 & 16 & 0 & 0 \\
\hline Total Oxalate & $12 / 15 / 2012$ & 16.01 & 16 & 0.01 & 0.0625 \\
\hline Total Oxalate & $12 / 16 / 2012$ & 16.02 & 16 & 0.02 & 0.125 \\
\hline Total Oxalate & $12 / 17 / 2012$ & 16.02 & 16 & 0.02 & 0.125 \\
\hline Total Oxalate & $12 / 20 / 2012$ & 16.22 & 16 & 0.22 & 1.375 \\
\hline Total Oxalate & $12 / 20 / 2012$ & 16.38 & 16 & 0.38 & 2.375 \\
\hline Total Oxalate & $12 / 21 / 2012$ & 15.19 & 16 & -0.81 & -5.0625 \\
\hline Total Oxalate & $12 / 21 / 2012$ & 16.49 & 16 & 0.49 & 3.0625 \\
\hline Total Oxalate & $12 / 23 / 2012$ & 14.75 & 16 & -1.25 & -7.8125 \\
\hline Total Oxalate & $12 / 23 / 2012$ & 14.88 & 16 & -1.12 & -7 \\
\hline Total Oxalate & $12 / 23 / 2012$ & 15.74 & 16 & -0.26 & -1.625 \\
\hline Total Oxalate & $12 / 23 / 2012$ & 15.74 & 16 & -0.26 & -1.625 \\
\hline Total Oxalate & $12 / 24 / 2012$ & 14.66 & 16 & -1.34 & -8.375 \\
\hline Total Oxalate & $12 / 25 / 2012$ & 14.4 & 16 & -1.6 & -10 \\
\hline Total Oxalate & $12 / 27 / 2012$ & 15.99 & 16 & -0.01 & -0.0625 \\
\hline Total Oxalate & $12 / 28 / 2012$ & 16.03 & 16 & 0.03 & 0.1875 \\
\hline Total Oxalate & $12 / 29 / 2012$ & 16.04 & 16 & 0.04 & 0.25 \\
\hline Total Oxalate & $12 / 30 / 2012$ & 16.1 & 16 & 0.1 & 0.625 \\
\hline Total Oxalate & $12 / 31 / 2012$ & 16.07 & 16 & 0.07 & 0.4375 \\
\hline Total Oxalate & $12 / 31 / 2012$ & 16.08 & 16 & 0.08 & 0.5 \\
\hline Total Oxalate & 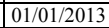 & 16.11 & 16 & 0.11 & 0.6875 \\
\hline Total Oxalate & $01 / 01 / 2013$ & 16.12 & 16 & 0.12 & 0.75 \\
\hline Total Oxalate & $01 / 02 / 2013$ & 16.13 & 16 & 0.13 & 0.8125 \\
\hline Total Oxalate & 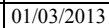 & 15.67 & 16 & -0.33 & -2.0625 \\
\hline Total Oxalate & $01 / 03 / 2013$ & 15.97 & 16 & -0.03 & -0.1875 \\
\hline Total Oxalate & $01 / 04 / 2013$ & 15.72 & 16 & -0.28 & -1.75 \\
\hline Total Oxalate & $01 / 05 / 2013$ & 15.83 & 16 & -0.17 & -1.0625 \\
\hline Total Oxalate & $01 / 05 / 2013$ & 15.83 & 16 & -0.17 & -1.0625 \\
\hline Total Oxalate & $01 / 06 / 2013$ & 15.81 & 16 & -0.19 & -1.1875 \\
\hline Total Oxalate & $01 / 07 / 2013$ & 15.99 & 16 & -0.01 & -0.0625 \\
\hline Total Oxalate & $01 / 08 / 2013$ & 15.74 & 16 & -0.26 & -1.625 \\
\hline Total Oxalate & $01 / 08 / 2013$ & 16.18 & 16 & 0.18 & 1.125 \\
\hline Total Oxalate & $01 / 09 / 2013$ & 16.74 & 16 & 0.74 & 4.625 \\
\hline Total Oxalate & $01 / 10 / 2013$ & 15.91 & 16 & -0.09 & -0.5625 \\
\hline Total Oxalate & $01 / 11 / 2013$ & 15.9 & 16 & -0.1 & -0.625 \\
\hline Total Oxalate & $01 / 11 / 2013$ & 17 & 16 & 1 & 6.25 \\
\hline Total Oxalate & $01 / 12 / 2013$ & 16.77 & 16 & 0.77 & 4.8125 \\
\hline Total Oxalate & $01 / 13 / 2013$ & 16.04 & 16 & 0.04 & 0.25 \\
\hline Total Oxalate & $01 / 13 / 2013$ & 16.45 & 16 & 0.45 & 2.8125 \\
\hline Total Oxalate & $01 / 13 / 2013$ & 16.52 & 16 & 0.52 & 3.25 \\
\hline Total Oxalate & $01 / 14 / 2013$ & 16.45 & 16 & 0.45 & 2.8125 \\
\hline Total Oxalate & $01 / 14 / 2013$ & 16.7 & 16 & 0.7 & 4.375 \\
\hline Total Oxalate & $01 / 14 / 2013$ & 16.85 & 16 & 0.85 & 5.3125 \\
\hline Total Oxalate & $01 / 14 / 2013$ & 17.23 & 16 & 1.23 & 7.6875 \\
\hline Total Oxalate & $01 / 15 / 2013$ & 16.83 & 16 & 0.83 & 5.1875 \\
\hline Total Oxalate & $01 / 16 / 2013$ & 16.74 & 16 & 0.74 & 4.625 \\
\hline Total Oxalate & $01 / 16 / 2013$ & 16.75 & 16 & 0.75 & 4.6875 \\
\hline Total Oxalate & $01 / 17 / 2013$ & 16.85 & 16 & 0.85 & 5.3125 \\
\hline Total Oxalate & $01 / 17 / 2013$ & 16.88 & 16 & 0.88 & 5.5 \\
\hline Total Oxalate & $01 / 18 / 2013$ & 22.26 & 16 & 6.26 & 39.125 \\
\hline Total Oxalate & $01 / 19 / 2013$ & 16.87 & 16 & 0.87 & 5.4375 \\
\hline Total Oxalate & 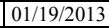 & 17.79 & 16 & 1.79 & 11.1875 \\
\hline Total Oxalate & $01 / 20 / 2013$ & 17.19 & 16 & 1.19 & 7.4375 \\
\hline
\end{tabular}

\begin{tabular}{|c|c|c|c|c|c|}
\hline Analyte & Date & $\begin{array}{c}\text { Measurement } \\
\text { (ppm) }\end{array}$ & $\begin{array}{c}\text { Reference } \\
\text { Value (ppm) }\end{array}$ & diff & $\begin{array}{l}\text { \% rel } \\
\text { diff }\end{array}$ \\
\hline Total Oxalate & $01 / 20 / 2013$ & 17.58 & 16 & 1.58 & 9.875 \\
\hline Total Oxalate & $01 / 21 / 2013$ & 16.91 & 16 & 0.91 & 5.6875 \\
\hline Total Oxalate & $01 / 21 / 2013$ & 17.38 & 16 & 1.38 & 8.625 \\
\hline Total Oxalate & \begin{tabular}{|l|}
$01 / 22 / 2013$ \\
\end{tabular} & 17.12 & 16 & 1.12 & 7 \\
\hline Total Oxalate & $01 / 22 / 2013$ & 17.12 & 16 & 1.12 & 7 \\
\hline Total Oxalate & $01 / 23 / 2013$ & 17.17 & 16 & 1.17 & 7.3125 \\
\hline Total Oxalate & $01 / 23 / 2013$ & 17.27 & 16 & 1.27 & 7.9375 \\
\hline Total Oxalate & $01 / 24 / 2013$ & 17.43 & 16 & 1.43 & 8.9375 \\
\hline Total Oxalate & $01 / 24 / 2013$ & 17.56 & 16 & 1.56 & 9.75 \\
\hline Total Oxalate & $01 / 25 / 2013$ & 16.15 & 16 & 0.15 & 0.9375 \\
\hline Total Oxalate & $01 / 25 / 2013$ & 16.15 & 16 & 0.15 & 0.9375 \\
\hline Total Oxalate & $01 / 26 / 2013$ & 15.94 & 16 & -0.06 & -0.375 \\
\hline Total Oxalate & $01 / 26 / 2013$ & 15.97 & 16 & -0.03 & -0.1875 \\
\hline Total Oxalate & $01 / 26 / 2013$ & 15.98 & 16 & \begin{tabular}{|l|}
-0.02 \\
\end{tabular} & -0.125 \\
\hline Total Oxalate & $01 / 26 / 2013$ & 16 & 16 & 0 & 0 \\
\hline Total Oxalate & $01 / 26 / 2013$ & 16.02 & 16 & 0.02 & 0.125 \\
\hline Total Oxalate & $01 / 27 / 2013$ & 16.03 & 16 & 0.03 & 0.1875 \\
\hline Total Oxalate & $01 / 28 / 2013$ & 16.01 & 16 & 0.01 & 0.0625 \\
\hline Total Oxalate & $01 / 28 / 2013$ & 16.01 & 16 & 0.01 & 0.0625 \\
\hline Total Oxalate & $01 / 28 / 2013$ & 16.02 & 16 & 0.02 & 0.125 \\
\hline Total Oxalate & $01 / 29 / 2013$ & 15.52 & 16 & -0.48 & -3 \\
\hline Total Oxalate & $01 / 30 / 2013$ & 14.82 & 16 & -1.18 & -7.375 \\
\hline Total Oxalate & $01 / 31 / 2013$ & 15 & 16 & -1 & -6.25 \\
\hline Total Oxalate & \begin{tabular}{|l|}
$02 / 01 / 2013$ \\
\end{tabular} & 15.43 & 16 & -0.57 & -3.5625 \\
\hline Total Oxalate & $02 / 01 / 2013$ & 15.43 & 16 & -0.57 & -3.5625 \\
\hline Total Oxalate & $02 / 01 / 2013$ & 15.46 & 16 & -0.54 & -3.375 \\
\hline Total Oxalate & $02 / 01 / 2013$ & 15.53 & 16 & -0.47 & -2.9375 \\
\hline Total Oxalate & $02 / 02 / 2013$ & 15.14 & 16 & \begin{tabular}{|l|}
-0.86 \\
\end{tabular} & -5.375 \\
\hline Total Oxalate & $02 / 03 / 2013$ & 15.6 & 16 & -0.4 & -2.5 \\
\hline Total Oxalate & \begin{tabular}{|l|}
$02 / 04 / 2013$ \\
\end{tabular} & 15.62 & 16 & \begin{tabular}{|l|}
-0.38 \\
\end{tabular} & -2.375 \\
\hline Total Oxalate & \begin{tabular}{|l|}
$02 / 04 / 2013$ \\
\end{tabular} & 15.63 & 16 & -0.37 & -2.3125 \\
\hline Total Oxalate & $02 / 05 / 2013$ & 15.06 & 16 & -0.94 & -5.875 \\
\hline Total Oxalate & \begin{tabular}{|l|}
$02 / 05 / 2013$ \\
\end{tabular} & 15.73 & 16 & \begin{tabular}{|l|}
-0.27 \\
\end{tabular} & -1.6875 \\
\hline Total Oxalate & $02 / 06 / 2013$ & 15.14 & 16 & -0.86 & -5.375 \\
\hline Total Oxalate & $02 / 07 / 2013$ & 15.71 & 16 & -0.29 & -1.8125 \\
\hline Total Oxalate & \begin{tabular}{|l|}
$02 / 07 / 2013$ \\
\end{tabular} & 15.79 & 16 & -0.21 & -1.3125 \\
\hline Total Oxalate & $02 / 08 / 2013$ & 15.57 & 16 & -0.43 & -2.6875 \\
\hline Total Oxalate & $02 / 09 / 2013$ & 15.03 & 16 & -0.97 & -6.0625 \\
\hline Total Oxalate & $02 / 09 / 2013$ & 15.45 & 16 & -0.55 & -3.4375 \\
\hline Total Oxalate & \begin{tabular}{|l|}
$02 / 09 / 2013$ \\
\end{tabular} & 15.81 & 16 & \begin{tabular}{|l|}
-0.19 \\
\end{tabular} & -1.1875 \\
\hline Total Oxalate & $02 / 10 / 2013$ & 15.61 & 16 & \begin{tabular}{|l|}
-0.39 \\
\end{tabular} & -2.4375 \\
\hline Formate & $11 / 09 / 2012$ & 2.09 & 2 & \begin{tabular}{|l|}
0.09 \\
\end{tabular} & 4.5 \\
\hline Formate & $11 / 10 / 2012$ & 2.05 & 2 & 0.05 & 2.5 \\
\hline Formate & $11 / 11 / 2012$ & 2.03 & 2 & 0.03 & 1.5 \\
\hline Formate & $11 / 13 / 2012$ & 2.03 & 2 & 0.03 & 1.5 \\
\hline Formate & $11 / 13 / 2012$ & 2.05 & 2 & 0.05 & 2.5 \\
\hline Formate & $11 / 14 / 2012$ & 2.02 & 2 & 0.02 & 1 \\
\hline Formate & \begin{tabular}{|l|}
$11 / 16 / 2012$ \\
\end{tabular} & 2.02 & 2 & 0.02 & 1 \\
\hline Formate & $11 / 16 / 2012$ & 2.07 & 2 & 0.07 & 3.5 \\
\hline Formate & $11 / 17 / 2012$ & 2.01 & 2 & 0.01 & 0.5 \\
\hline Formate & $11 / 18 / 2012$ & 2.02 & 2 & 0.02 & 1 \\
\hline Formate & $11 / 19 / 2012$ & 2.03 & 2 & 0.03 & 1.5 \\
\hline Formate & $11 / 20 / 2012$ & 2.03 & 2 & \begin{tabular}{|l|}
0.03 \\
\end{tabular} & 1.5 \\
\hline Formate & $11 / 21 / 2012$ & 1.92 & 2 & -0.08 & -4 \\
\hline Formate & $11 / 21 / 2012$ & 2.06 & 2 & 0.06 & 3 \\
\hline Formate & $11 / 21 / 2012$ & 2.06 & 2 & 0.06 & 3 \\
\hline Formate & $11 / 22 / 2012$ & 1.95 & 2 & -0.05 & -2.5 \\
\hline Formate & $11 / 24 / 2012$ & 1.9 & 2 & \begin{tabular}{|l|}
-0.1 \\
\end{tabular} & -5 \\
\hline Formate & $11 / 24 / 2012$ & 1.96 & 2 & \begin{tabular}{|l|}
-0.04 \\
\end{tabular} & -2 \\
\hline Formate & \begin{tabular}{|l|}
$11 / 24 / 2012$ \\
\end{tabular} & 2.01 & 2 & 0.01 & 0.5 \\
\hline Formate & $11 / 24 / 2012$ & 2.01 & 2 & 0.01 & 0.5 \\
\hline Formate & $11 / 25 / 2012$ & 1.97 & 2 & -0.03 & -1.5 \\
\hline Formate & $11 / 25 / 2012$ & 1.99 & 2 & \begin{tabular}{|l|}
-0.01 \\
\end{tabular} & -0.5 \\
\hline Formate & $11 / 25 / 2012$ & 1.99 & 2 & \begin{tabular}{|l|}
-0.01 \\
\end{tabular} & -0.5 \\
\hline Formate & $11 / 25 / 2012$ & 2.2 & 2 & 0.2 & 10 \\
\hline Formate & $11 / 26 / 2012$ & 2.17 & 2 & 0.17 & 8.5 \\
\hline Formate & $11 / 26 / 2012$ & 2.17 & 2 & 0.17 & 8.5 \\
\hline Formate & $11 / 27 / 2012$ & 2.21 & 2 & 0.21 & 10.5 \\
\hline Formate & $11 / 28 / 2012$ & 2.19 & 2 & 0.19 & 9.5 \\
\hline Formate & $11 / 28 / 2012$ & 2.21 & 2 & 0.21 & 10.5 \\
\hline Formate & $11 / 28 / 2012$ & 2.21 & 2 & 0.21 & 10.5 \\
\hline Formate & $11 / 29 / 2012$ & 2.2 & 2 & 0.2 & 10 \\
\hline Formate & \begin{tabular}{|l|}
$11 / 30 / 2012$ \\
\end{tabular} & 2.13 & 2 & 0.13 & 6.5 \\
\hline Formate & $12 / 02 / 2012$ & 2.04 & 2 & 0.04 & 2 \\
\hline Formate & $12 / 02 / 2012$ & 2.06 & 2 & 0.06 & 3 \\
\hline Formate & $12 / 03 / 2012$ & 2.07 & 2 & 0.07 & 3.5 \\
\hline Formate & $12 / 03 / 2012$ & 2.07 & 2 & 0.07 & 3.5 \\
\hline Formate & $12 / 04 / 2012$ & 1.97 & 2 & -0.03 & -1.5 \\
\hline Formate & \begin{tabular}{|l|}
$12 / 04 / 2012$ \\
\end{tabular} & 2.06 & 2 & 0.06 & 3 \\
\hline Formate & $12 / 06 / 2012$ & 1.88 & 2 & -0.12 & -6 \\
\hline Formate & $12 / 06 / 2012$ & 2.06 & 2 & 0.06 & 3 \\
\hline Formate & $12 / 07 / 2012$ & 1.94 & 2 & -0.06 & -3 \\
\hline Formate & $12 / 08 / 2012$ & 2.07 & 2 & \begin{tabular}{|l|}
0.07 \\
\end{tabular} & 3.5 \\
\hline
\end{tabular}


SRNL-STI-2013-00139

Revision 0

Table A1. DWPF Laboratory Measurements of Anion and TOC Standards

\begin{tabular}{|c|c|c|c|c|c|}
\hline Analyte & Date & \begin{tabular}{|c|}
$\begin{array}{c}\text { Measurement } \\
(\mathrm{ppm})\end{array}$ \\
\end{tabular} & $\begin{array}{c}\text { Reference } \\
\text { Value (ppm) }\end{array}$ & diff & $\begin{array}{l}\text { \% rel } \\
\text { diff }\end{array}$ \\
\hline Formate & $12 / 09 / 2012$ & $\begin{array}{r}1.9 \\
\end{array}$ & 2 & -0.1 & -5 \\
\hline Formate & $12 / 09 / 2012$ & 2.04 & 2 & 0.04 & 2 \\
\hline Formate & $12 / 09 / 2012$ & 2.06 & 2 & 0.06 & 3 \\
\hline Formate & $12 / 10 / 2012$ & 2.06 & 2 & 0.06 & 3 \\
\hline Formate & $12 / 11 / 2012$ & 1.96 & 2 & -0.04 & -2 \\
\hline Formate & $12 / 12 / 2012$ & 1.91 & 2 & \begin{tabular}{|l|} 
\\
\end{tabular} & -4.5 \\
\hline Formate & $12 / 12 / 2012$ & 1.98 & 2 & -0.02 & -1 \\
\hline Formate & $12 / 14 / 2012$ & 2.07 & 2 & \begin{tabular}{|l|}
0.07 \\
\end{tabular} & 3.5 \\
\hline Formate & $12 / 14 / 2012$ & 2.07 & 2 & 0.07 & 3.5 \\
\hline Formate & $12 / 15 / 2012$ & 2.01 & 2 & 0.01 & 0.5 \\
\hline Formate & $12 / 15 / 2012$ & 2.04 & 2 & 0.04 & 2 \\
\hline Formate & $12 / 15 / 2012$ & 2.04 & 2 & 0.04 & $\overline{2}$ \\
\hline Formate & $12 / 16 / 2012$ & 2.05 & 2 & 0.05 & 2.5 \\
\hline Formate & $12 / 17 / 2012$ & 1.94 & 2 & -0.06 & -3 \\
\hline Formate & $12 / 17 / 2012$ & 2.04 & 2 & 0.04 & 2 \\
\hline Formate & $12 / 17 / 2012$ & 2.05 & 2 & 0.05 & 2.5 \\
\hline Formate & $12 / 18 / 2012$ & 2.02 & 2 & 0.02 & 1 \\
\hline Formate & $12 / 20 / 2012$ & 2.06 & 2 & 0.06 & $\overline{3}$ \\
\hline Formate & $12 / 20 / 2012$ & 2.07 & 2 & 0.07 & 3.5 \\
\hline Formate & $12 / 21 / 2012$ & 2.03 & 2 & 0.03 & 1.5 \\
\hline Formate & $12 / 21 / 2012$ & 2.05 & 2 & 0.05 & 2.5 \\
\hline Formate & $12 / 22 / 2012$ & 2.05 & 2 & 0.05 & 2.5 \\
\hline Formate & $12 / 23 / 2012$ & 2.03 & 2 & 0.03 & 1.5 \\
\hline Formate & $12 / 23 / 2012$ & 2.04 & 2 & 0.04 & 2 \\
\hline Formate & $12 / 23 / 2012$ & 2.06 & 2 & 0.06 & $\overline{3}$ \\
\hline Formate & $12 / 23 / 2012$ & 2.06 & 2 & 0.06 & 3 \\
\hline Formate & $12 / 24 / 2012$ & 2 & 2 & 0 & 0 \\
\hline Formate & $12 / 24 / 2012$ & 2.07 & 2 & 0.07 & 3.5 \\
\hline Formate & $12 / 25 / 2012$ & 1.85 & 2 & -0.15 & -7.5 \\
\hline Formate & $12 / 25 / 2012$ & 1.85 & 2 & -0.15 & -7.5 \\
\hline Formate & $12 / 27 / 2012$ & 2.06 & 2 & 0.06 & 3 \\
\hline Formate & $12 / 27 / 2012$ & 2.06 & 2 & 0.06 & 3 \\
\hline Formate & $12 / 27 / 2012$ & 2.07 & 2 & 0.07 & 3.5 \\
\hline Formate & $12 / 28 / 2012$ & 1.91 & 2 & -0.09 & -4.5 \\
\hline Formate & $12 / 28 / 2012$ & 2.06 & 2 & 0.06 & 3 \\
\hline Formate & $12 / 28 / 2012$ & 2.06 & 2 & 0.06 & 3 \\
\hline Formate & $12 / 29 / 2012$ & 2.05 & 2 & 0.05 & 2.5 \\
\hline Formate & $12 / 29 / 2012$ & 2.05 & 2 & 0.05 & 2.5 \\
\hline Formate & $12 / 29 / 2012$ & 2.07 & 2 & 0.07 & 3.5 \\
\hline Formate & $12 / 30 / 2012$ & 2.06 & 2 & 0.06 & 3 \\
\hline Formate & $12 / 31 / 2012$ & 2.02 & 2 & 0.02 & 1 \\
\hline Formate & $12 / 31 / 2012$ & 2.06 & 2 & 0.06 & 3 \\
\hline Formate & $01 / 01 / 2013$ & 2.05 & 2 & 0.05 & 2.5 \\
\hline Formate & $01 / 01 / 2013$ & 2.07 & 2 & 0.07 & 3.5 \\
\hline Formate & $01 / 02 / 2013$ & 2.08 & 2 & 0.08 & 4 \\
\hline Formate & $01 / 03 / 2013$ & 2.04 & 2 & 0.04 & 2 \\
\hline Formate & \begin{tabular}{|l|l}
$01 / 03 / 2013$ \\
\end{tabular} & 2.04 & 2 & 0.04 & 2 \\
\hline Formate & $01 / 04 / 2013$ & 2.02 & 2 & 0.02 & $\overline{1}$ \\
\hline Formate & $01 / 05 / 2013$ & 2.06 & 2 & 0.06 & 3 \\
\hline Formate & $01 / 05 / 2013$ & 2.06 & 2 & 0.06 & $\overline{3}$ \\
\hline Formate & $01 / 06 / 2013$ & 2 & 2 & 0 & 50 \\
\hline Formate & $01 / 07 / 2013$ & 1.83 & 2 & -0.17 & -8.5 \\
\hline Formate & $01 / 08 / 2013$ & 2.01 & 2 & 0.01 & 0.5 \\
\hline Formate & $01 / 11 / 2013$ & 1.89 & 2 & -0.11 & -5.5 \\
\hline Formate & $01 / 11 / 2013$ & 2.07 & 2 & 0.07 & 3.5 \\
\hline Formate & $01 / 12 / 2013$ & 1.88 & 2 & -0.12 & -6 \\
\hline Formate & $01 / 12 / 2013$ & 2 & 2 & 0 & 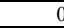 \\
\hline Formate & $01 / 13 / 2013$ & 1.98 & 2 & -0.02 & -1 \\
\hline Formate & $01 / 13 / 2013$ & 2.01 & 2 & 0.01 & 0.5 \\
\hline Formate & \begin{tabular}{|l|l}
$01 / 13 / 2013$ \\
\end{tabular} & 2.06 & 2 & \begin{tabular}{|l}
0.06 \\
\end{tabular} & 3 \\
\hline Formate & $01 / 14 / 2013$ & 1.93 & 2 & -0.07 & -3.5 \\
\hline Formate & $01 / 14 / 2013$ & 1.93 & 2 & -0.07 & -3.5 \\
\hline Formate & $01 / 14 / 2013$ & 1.98 & 2 & -0.02 & -1 \\
\hline Formate & $01 / 14 / 2013$ & 2 & 2 & 0 & 0 \\
\hline Formate & $01 / 14 / 2013$ & 2.05 & 2 & 0.05 & 2.5 \\
\hline Formate & $01 / 15 / 2013$ & 2.07 & 2 & 0.07 & 3.5 \\
\hline Formate & 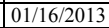 & 2.06 & 2 & 0.06 & 3 \\
\hline Formate & $01 / 17 / 2013$ & 2.1 & 2 & 0.1 & 5 \\
\hline Formate & $01 / 18 / 2013$ & 2.07 & 2 & 0.07 & 3.5 \\
\hline Formate & 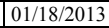 & 2.07 & 2 & 0.07 & 3.5 \\
\hline Formate & $01 / 18 / 2013$ & 2.15 & 2 & 0.15 & 7.5 \\
\hline Formate & $01 / 18 / 2013$ & 2.15 & 2 & 0.15 & 7.5 \\
\hline Formate & \begin{tabular}{|l|l|l|}
$01 / 2013$ \\
\end{tabular} & 2.15 & 2 & \begin{tabular}{|l|l|}
0.15 \\
\end{tabular} & 7.5 \\
\hline Formate & $01 / 19 / 2013$ & 2.05 & 2 & 0.05 & 2.5 \\
\hline Formate & $01 / 19 / 2013$ & 2.13 & 2 & 0.13 & 6.5 \\
\hline Formate & 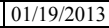 & 2.14 & 2 & 0.14 & 7 \\
\hline Formate & $01 / 20 / 2013$ & 2.1 & 2 & 0.1 & 5 \\
\hline Formate & $01 / 21 / 2013$ & 2.06 & 2 & 0.06 & 3 \\
\hline Formate & $01 / 21 / 2013$ & 2.13 & 2 & 0.13 & 6.5 \\
\hline Formate & $01 / 21 / 2013$ & 2.13 & 2 & 0.13 & 6.5 \\
\hline Formate & $01 / 22 / 2013$ & 2.04 & 2 & \begin{tabular}{|l|l|} 
& 0.04 \\
\end{tabular} & 2 \\
\hline Formate & $01 / 22 / 2013$ & 2.07 & 2 & \begin{tabular}{|l|}
0.07 \\
\end{tabular} & 3.5 \\
\hline Formate & $01 / 22 / 2013$ & 2.11 & 2 & 0.11 & 5.5 \\
\hline
\end{tabular}

\begin{tabular}{|c|c|c|c|c|c|}
\hline Analyte & Date & \begin{tabular}{|c|}
$\begin{array}{c}\text { Measurement } \\
\text { (ppm) }\end{array}$ \\
\end{tabular} & $\begin{array}{c}\text { Reference } \\
\text { Value (ppm) }\end{array}$ & diff & $\begin{array}{l}\text { \% rel } \\
\text { diff }\end{array}$ \\
\hline Formate & $01 / 23 / 2013$ & 2.03 & 2 & 0.03 & 1.5 \\
\hline Formate & $01 / 23 / 2013$ & 2.03 & 2 & 0.03 & 1.5 \\
\hline Formate & $01 / 23 / 2013$ & 2.04 & 2 & 0.04 & 2 \\
\hline Formate & $01 / 23 / 2013$ & 2.05 & 2 & 0.05 & 2.5 \\
\hline Formate & $01 / 24 / 2013$ & 2.04 & 2 & 0.04 & 2 \\
\hline Formate & $01 / 24 / 2013$ & 2.06 & 2 & 0.06 & 3 \\
\hline Formate & $01 / 25 / 2013$ & 2.04 & 2 & 0.04 & 2 \\
\hline Formate & $01 / 25 / 2013$ & 2.09 & 2 & 0.09 & 4.5 \\
\hline Formate & $01 / 25 / 2013$ & 2.11 & 2 & 0.11 & 5.5 \\
\hline Formate & $01 / 26 / 2013$ & 2.12 & 2 & 0.12 & 6 \\
\hline Formate & $01 / 26 / 2013$ & 2.12 & 2 & 0.12 & 6 \\
\hline Formate & $01 / 26 / 2013$ & 2.12 & 2 & 0.12 & 6 \\
\hline Formate & 01/26/2013 & 2.13 & 2 & 0.13 & 6.5 \\
\hline Formate & $01 / 26 / 2013$ & 2.14 & 2 & 0.14 & 7 \\
\hline Formate & $01 / 27 / 2013$ & 2.12 & 2 & 0.12 & 6 \\
\hline Formate & $01 / 27 / 2013$ & 2.13 & 2 & 0.13 & 6.5 \\
\hline Formate & $01 / 28 / 2013$ & 2.01 & 2 & 0.01 & 0.5 \\
\hline Formate & $01 / 28 / 2013$ & 2.12 & 2 & 0.12 & 6 \\
\hline Formate & $01 / 28 / 2013$ & 2.12 & 2 & 0.12 & 6 \\
\hline Formate & $01 / 29 / 2013$ & 2.08 & 2 & 0.08 & 4 \\
\hline Formate & $01 / 29 / 2013$ & 2.12 & 2 & 0.12 & 6 \\
\hline Formate & $01 / 30 / 2013$ & 2.08 & 2 & 0.08 & 4 \\
\hline Formate & $01 / 31 / 2013$ & 2.08 & 2 & 0.08 & 4 \\
\hline Formate & $01 / 31 / 2013$ & 2.1 & 2 & 0.1 & 5 \\
\hline Formate & $01 / 31 / 2013$ & 2.11 & 2 & 0.11 & 5.5 \\
\hline Formate & \begin{tabular}{|l|l}
$02 / 01 / 2013$ \\
\end{tabular} & 1.97 & 2 & -0.03 & -1.5 \\
\hline Formate & $02 / 01 / 2013$ & 2 & 2 & 0 & 0 \\
\hline Formate & $02 / 01 / 2013$ & 2 & 2 & 0 & $\overline{0}$ \\
\hline Formate & $02 / 01 / 2013$ & 2.01 & 2 & 0.01 & 0.5 \\
\hline Formate & $02 / 01 / 2013$ & 2.08 & 2 & 0.08 & 4 \\
\hline Formate & \begin{tabular}{|l|l|}
$02 / 01 / 2013$ \\
\end{tabular} & 2.08 & 2 & 0.08 & 4 \\
\hline Formate & \begin{tabular}{|l|l|}
$02 / 02 / 2013$ \\
\end{tabular} & 2.09 & 2 & 0.09 & 4.5 \\
\hline Formate & \begin{tabular}{|l|l|}
$02 / 03 / 2013$ \\
\end{tabular} & 2.04 & 2 & 0.04 & 2 \\
\hline Formate & $02 / 03 / 2013$ & 2.04 & 2 & 0.04 & 2 \\
\hline Formate & $02 / 03 / 2013$ & 2.1 & 2 & 0.1 & 5 \\
\hline Formate & $02 / 04 / 2013$ & 1.98 & 2 & -0.02 & -1 \\
\hline Formate & \begin{tabular}{|l|l|}
$02 / 04 / 2013$ \\
\end{tabular} & 2.03 & 2 & \begin{tabular}{|l|}
0.03 \\
\end{tabular} & 1.5 \\
\hline Formate & $02 / 05 / 2013$ & 1.99 & 2 & -0.01 & -0.5 \\
\hline Formate & $02 / 05 / 2013$ & 2 & 2 & 0 & 0 \\
\hline Formate & $02 / 06 / 2013$ & 2.06 & 2 & 0.06 & 3 \\
\hline Formate & $02 / 07 / 2013$ & 2.06 & 2 & 0.06 & 3 \\
\hline Formate & $02 / 07 / 2013$ & 2.09 & 2 & 0.09 & 4.5 \\
\hline Formate & $02 / 08 / 2013$ & 2.07 & 2 & 0.07 & 3.5 \\
\hline Formate & $02 / 09 / 2013$ & 2.06 & 2 & 0.06 & 3 \\
\hline Formate & \begin{tabular}{|l|}
$02 / 09 / 2013$ \\
\end{tabular} & 2.07 & 2 & 0.07 & 3.5 \\
\hline Formate & $02 / 09 / 2013$ & 2.09 & 2 & 0.09 & 4.5 \\
\hline Formate & $02 / 10 / 2013$ & 2.08 & 2 & 0.08 & 4 \\
\hline Formate & $11 / 09 / 2012$ & 16.47 & 16 & 0.47 & 2.9375 \\
\hline Formate & $11 / 10 / 2012$ & 16.09 & 16 & 0.09 & 0.5625 \\
\hline Formate & \begin{tabular}{|l|l|}
$11 / 11 / 2012$ \\
\end{tabular} & 16.02 & 16 & 0.02 & 0.125 \\
\hline Formate & $11 / 13 / 2012$ & 16.09 & 16 & 0.09 & 0.5625 \\
\hline Formate & 11/13/2012 & 16.14 & 16 & 0.14 & 0.875 \\
\hline Formate & $11 / 14 / 2012$ & 15.94 & 16 & \begin{tabular}{|l|}
-0.06 \\
\end{tabular} & -0.375 \\
\hline Formate & $11 / 16 / 2012$ & 16.13 & 16 & \begin{tabular}{|l|}
0.13 \\
\end{tabular} & 0.8125 \\
\hline Formate & $11 / 16 / 2012$ & 16.44 & 16 & 0.44 & 2.75 \\
\hline Formate & $11 / 17 / 2012$ & 16.38 & 16 & 0.38 & 2.375 \\
\hline Formate & $11 / 18 / 2012$ & 16.12 & 16 & 0.12 & 0.75 \\
\hline Formate & $11 / 19 / 2012$ & 16.25 & 16 & 0.25 & 1.5625 \\
\hline Formate & $11 / 20 / 2012$ & 16.22 & 16 & 0.22 & 1.375 \\
\hline Formate & $11 / 21 / 2012$ & 15.2 & 16 & -0.8 & -5 \\
\hline Formate & $11 / 21 / 2012$ & 16.23 & 16 & 0.23 & 1.4375 \\
\hline Formate & $11 / 21 / 2012$ & 16.39 & 16 & \begin{tabular}{|l|} 
\\
\end{tabular} & 2.4375 \\
\hline Formate & $11 / 22 / 2012$ & 15.64 & 16 & -0.36 & -2.25 \\
\hline Formate & $11 / 24 / 2012$ & 15.7 & 16 & \begin{tabular}{|l|} 
\\
\end{tabular} & -1.875 \\
\hline Formate & $11 / 24 / 2012$ & 16.16 & 16 & 0.16 & 1 \\
\hline Formate & $11 / 24 / 2012$ & 16.35 & 16 & 0.35 & 2.1875 \\
\hline Formate & $11 / 24 / 2012$ & 16.39 & 16 & \begin{tabular}{|l|}
0.39 \\
\end{tabular} & 2.4375 \\
\hline Formate & $11 / 25 / 2012$ & 15.88 & 16 & $\mid-0.12$ & -0.75 \\
\hline Formate & $11 / 25 / 2012$ & 15.88 & 16 & -0.12 & -0.75 \\
\hline Formate & $11 / 25 / 2012$ & 16.34 & 16 & \begin{tabular}{|l|}
0.34 \\
\end{tabular} & 2.125 \\
\hline Formate & $11 / 25 / 2012$ & 16.5 & 16 & 0.5 & 3.125 \\
\hline Formate & $11 / 26 / 2012$ & 15.74 & 16 & -0.26 & -1.625 \\
\hline Formate & $11 / 26 / 2012$ & 16.16 & 16 & 0.16 & 1 \\
\hline Formate & $11 / 27 / 2012$ & 16.59 & 16 & 0.59 & 3.6875 \\
\hline Formate & $11 / 28 / 2012$ & 16.57 & 16 & 0.57 & 3.5625 \\
\hline Formate & $11 / 28 / 2012$ & 16.57 & 16 & 0.57 & 3.5625 \\
\hline Formate & $11 / 28 / 2012$ & 16.66 & 16 & 0.66 & 4.125 \\
\hline Formate & $11 / 29 / 2012$ & 16.57 & 16 & 0.57 & 3.5625 \\
\hline Formate & \begin{tabular}{|l|l|}
$11 / 30 / 2012$ \\
\end{tabular} & 15.86 & 16 & -0.14 & -0.875 \\
\hline Formate & $12 / 02 / 2012$ & 16.14 & 16 & 0.14 & 0.875 \\
\hline Formate & $12 / 02 / 2012$ & 16.27 & 16 & 0.27 & 1.6875 \\
\hline Formate & \begin{tabular}{|l|}
$12 / 03 / 2012$ \\
\end{tabular} & 15.84 & 16 & -0.16 & -1 \\
\hline Formate & $12 / 03 / 2012$ & 16.54 & 16 & \begin{tabular}{|l|}
0.54 \\
\end{tabular} & 3.375 \\
\hline
\end{tabular}


SRNL-STI-2013-00139

Revision 0

Table A1. DWPF Laboratory Measurements of Anion and TOC Standards

\begin{tabular}{|c|c|c|c|c|c|}
\hline Analyte & Date & $\begin{array}{c}\text { Measurement } \\
\text { (ppm) }\end{array}$ & $\begin{array}{c}\text { Reference } \\
\text { Value (ppm) }\end{array}$ & diff & $\begin{array}{l}\text { \% rel } \\
\text { diff }\end{array}$ \\
\hline Formate & $12 / 04 / 2012$ & 16.33 & 16 & 0.33 & 2.0625 \\
\hline Formate & $12 / 04 / 2012$ & 16.39 & 16 & 0.39 & 2.4375 \\
\hline Formate & $12 / 06 / 2012$ & 16.55 & 16 & 0.55 & 3.4375 \\
\hline Formate & $12 / 06 / 2012$ & 16.64 & 16 & 0.64 & 4 \\
\hline Formate & $12 / 07 / 2012$ & 14.91 & 16 & -1.09 & -6.8125 \\
\hline Formate & $12 / 08 / 2012$ & 16.11 & 16 & 0.11 & 0.6875 \\
\hline Formate & $12 / 09 / 2012$ & 16.22 & 16 & 0.22 & 1.375 \\
\hline Formate & $12 / 09 / 2012$ & 16.55 & 16 & 0.55 & 3.4375 \\
\hline Formate & $12 / 09 / 2012$ & 16.56 & 16 & 0.56 & 3.5 \\
\hline Formate & $12 / 10 / 2012$ & 16.53 & 16 & 0.53 & 3.3125 \\
\hline Formate & $12 / 11 / 2012$ & 15.9 & 16 & $\begin{array}{ll}-0.1 \\
\end{array}$ & -0.625 \\
\hline Formate & $12 / 12 / 2012$ & 15.29 & 16 & \begin{tabular}{|l|}
-0.71 \\
\end{tabular} & -4.4375 \\
\hline Formate & $12 / 12 / 2012$ & 15.75 & 16 & -0.25 & -1.5625 \\
\hline Formate & $12 / 14 / 2012$ & 16.41 & 16 & \begin{tabular}{|l|}
0.41 \\
\end{tabular} & 2.5625 \\
\hline Formate & $12 / 14 / 2012$ & 16.43 & 16 & 0.43 & 2.6875 \\
\hline Formate & $12 / 15 / 2012$ & 16.23 & 16 & 0.23 & 1.4375 \\
\hline Formate & $12 / 15 / 2012$ & 16.23 & 16 & 0.23 & 1.4375 \\
\hline Formate & $12 / 15 / 2012$ & 16.24 & 16 & 0.24 & 1.5 \\
\hline Formate & $12 / 16 / 2012$ & 16.34 & 16 & 0.34 & 2.125 \\
\hline Formate & $12 / 17 / 2012$ & 16.29 & 16 & 0.29 & 1.8125 \\
\hline Formate & $12 / 17 / 2012$ & 16.3 & 16 & 0.3 & 1.875 \\
\hline Formate & $12 / 17 / 2012$ & 16.43 & 16 & 0.43 & 2.6875 \\
\hline Formate & $12 / 18 / 2012$ & 16.24 & 16 & 0.24 & 1.5 \\
\hline Formate & $12 / 20 / 2012$ & 15.96 & 16 & -0.04 & -0.25 \\
\hline Formate & $12 / 20 / 2012$ & $\begin{array}{ll}16.1 \\
\end{array}$ & 16 & 0.1 & 0.625 \\
\hline Formate & $12 / 21 / 2012$ & 16.29 & 16 & 0.29 & 1.8125 \\
\hline Formate & $12 / 21 / 2012$ & 16.36 & 16 & 0.36 & 2.25 \\
\hline Formate & $12 / 22 / 2012$ & 16.24 & 16 & 0.24 & 1.5 \\
\hline Formate & $12 / 23 / 2012$ & 15.64 & 16 & -0.36 & -2.25 \\
\hline Formate & $12 / 23 / 2012$ & 16.26 & 16 & 0.26 & 1.625 \\
\hline Formate & $12 / 23 / 2012$ & 16.26 & 16 & 0.26 & 1.625 \\
\hline Formate & $12 / 23 / 2012$ & 16.36 & 16 & 0.36 & 2.25 \\
\hline Formate & $12 / 24 / 2012$ & 16.31 & 16 & 0.31 & 1.9375 \\
\hline Formate & $12 / 24 / 2012$ & 16.46 & 16 & 0.46 & 2.875 \\
\hline Formate & $12 / 25 / 2012$ & 16.18 & 16 & 0.18 & 1.125 \\
\hline Formate & $12 / 25 / 2012$ & 16.18 & 16 & 0.18 & 1.125 \\
\hline Formate & 12/27/2012 & 16.41 & 16 & 0.41 & 2.5625 \\
\hline Formate & $12 / 27 / 2012$ & 16.41 & 16 & 0.41 & 2.5625 \\
\hline Formate & $12 / 27 / 2012$ & 16.48 & 16 & 0.48 & 3 \\
\hline Formate & $12 / 28 / 2012$ & 16 & 16 & 0 & 0 \\
\hline Formate & $12 / 28 / 2012$ & 16.43 & 16 & 0.43 & 2.6875 \\
\hline Formate & $12 / 28 / 2012$ & 16.43 & 16 & 0.43 & 2.6875 \\
\hline Formate & $12 / 29 / 2012$ & 16.17 & 16 & 0.17 & 1.0625 \\
\hline Formate & $12 / 29 / 2012$ & 16.38 & 16 & 0.38 & 2.375 \\
\hline Formate & $12 / 29 / 2012$ & 16.38 & 16 & 0.38 & 2.375 \\
\hline Formate & $12 / 30 / 2012$ & 16.42 & 16 & 0.42 & 2.625 \\
\hline Formate & $12 / 31 / 2012$ & 16.39 & 16 & 0.39 & 2.4375 \\
\hline Formate & $12 / 31 / 2012$ & 16.42 & 16 & 0.42 & 2.625 \\
\hline Formate & $01 / 01 / 2013$ & 16.32 & 16 & 0.32 & 2 \\
\hline Formate & $01 / 01 / 2013$ & 16.45 & 16 & 0.45 & 2.8125 \\
\hline Formate & $01 / 02 / 2013$ & 16.35 & 16 & 0.35 & 2.1875 \\
\hline Formate & $01 / 03 / 2013$ & 16.01 & 16 & 0.01 & 0.0625 \\
\hline Formate & $01 / 03 / 2013$ & 16.34 & 16 & 0.34 & 2.125 \\
\hline Formate & $01 / 04 / 2013$ & 16.02 & 16 & 0.02 & 0.125 \\
\hline Formate & $01 / 05 / 2013$ & 16 & 16 & 0 & 0 \\
\hline Formate & \begin{tabular}{|l|}
$01 / 05 / 2013$ \\
\end{tabular} & 16.27 & 16 & 0.27 & 1.6875 \\
\hline Formate & \begin{tabular}{|l|}
$01 / 06 / 2013$ \\
\end{tabular} & 15.77 & 16 & -0.23 & -1.4375 \\
\hline Formate & $01 / 07 / 2013$ & 15.67 & 16 & -0.33 & -2.0625 \\
\hline Formate & $01 / 08 / 2013$ & 15.92 & 16 & -0.08 & -0.5 \\
\hline Formate & $01 / 11 / 2013$ & 15.3 & 16 & \begin{tabular}{|l|}
-0.7 \\
\end{tabular} & -4.375 \\
\hline Formate & $01 / 11 / 2013$ & 16.43 & 16 & \begin{tabular}{|l|}
0.43 \\
\end{tabular} & 2.6875 \\
\hline Formate & $01 / 12 / 2013$ & 15.11 & 16 & -0.89 & -5.5625 \\
\hline Formate & $01 / 12 / 2013$ & 15.55 & 16 & -0.45 & -2.8125 \\
\hline Formate & $01 / 13 / 2013$ & 15.01 & 16 & \begin{tabular}{|l|}
-0.99 \\
\end{tabular} & -6.1875 \\
\hline Formate & $01 / 13 / 2013$ & 15.19 & 16 & -0.81 & -5.0625 \\
\hline Formate & $01 / 13 / 2013$ & 16.07 & 16 & \begin{tabular}{|l|}
0.07 \\
\end{tabular} & 0.4375 \\
\hline Formate & $01 / 14 / 2013$ & 15.09 & 16 & -0.91 & -5.6875 \\
\hline Formate & $01 / 14 / 2013$ & 15.09 & 16 & -0.91 & -5.6875 \\
\hline Formate & $01 / 14 / 2013$ & 15.24 & 16 & -0.76 & -4.75 \\
\hline Formate & $01 / 14 / 2013$ & 15.32 & 16 & \begin{tabular}{|l|}
-0.68 \\
\end{tabular} & -4.25 \\
\hline Formate & $01 / 14 / 2013$ & 15.37 & 16 & -0.63 & -3.9375 \\
\hline Formate & $01 / 15 / 2013$ & 15.27 & 16 & -0.73 & -4.5625 \\
\hline Formate & $01 / 16 / 2013$ & 16.2 & 16 & 0.2 & 1.25 \\
\hline Formate & $01 / 17 / 2013$ & 16.25 & 16 & 0.25 & 1.5625 \\
\hline Formate & \begin{tabular}{|l|}
$01 / 18 / 2013$ \\
\end{tabular} & 15.57 & 16 & -0.43 & -2.6875 \\
\hline Formate & $01 / 18 / 2013$ & 15.57 & 16 & \begin{tabular}{|l|}
-0.43 \\
\end{tabular} & -2.6875 \\
\hline Formate & $01 / 18 / 2013$ & 16.28 & 16 & 0.28 & 1.75 \\
\hline Formate & $01 / 18 / 2013$ & 16.28 & 16 & 0.28 & 1.75 \\
\hline Formate & $01 / 18 / 2013$ & 16.59 & 16 & 0.59 & 3.6875 \\
\hline Formate & $01 / 19 / 2013$ & 16.54 & 16 & 0.54 & 3.375 \\
\hline Formate & \begin{tabular}{|l|}
$01 / 19 / 2013$ \\
\end{tabular} & 16.57 & 16 & 0.57 & 3.5625 \\
\hline Formate & \begin{tabular}{|l|}
$01 / 20 / 2013$ \\
\end{tabular} & 16.32 & 16 & 0.32 & 2 \\
\hline Formate & $01 / 20 / 2013$ & 16.47 & 16 & 0.47 & 2.9375 \\
\hline
\end{tabular}

\begin{tabular}{|c|c|c|c|c|c|}
\hline Analyte & Date & \begin{tabular}{|c|}
$\begin{array}{c}\text { Measurement } \\
\text { (ppm) }\end{array}$ \\
\end{tabular} & $\begin{array}{c}\text { Reference } \\
\text { Value (ppm) }\end{array}$ & diff & $\begin{array}{c}\text { \% rel } \\
\text { diff }\end{array}$ \\
\hline Formate & $01 / 21 / 2013$ & $\begin{array}{r}16.11 \\
\end{array}$ & 16 & 0.11 & 0.6875 \\
\hline Formate & $01 / 21 / 2013$ & 16.19 & 16 & 0.19 & 1.1875 \\
\hline Formate & $01 / 21 / 2013$ & 16.25 & 16 & 0.25 & 1.5625 \\
\hline Formate & $01 / 22 / 2013$ & 16.25 & 16 & 0.25 & 1.5625 \\
\hline Formate & $01 / 22 / 2013$ & 16.28 & 16 & 0.28 & 1.75 \\
\hline Formate & $01 / 22 / 2013$ & 16.36 & 16 & 0.36 & 2.25 \\
\hline Formate & $01 / 23 / 2013$ & 16.13 & 16 & 0.13 & 0.8125 \\
\hline Formate & $01 / 23 / 2013$ & 16.16 & 16 & 0.16 & 1 \\
\hline Formate & $01 / 23 / 2013$ & 16.35 & 16 & 0.35 & 2.1875 \\
\hline Formate & $01 / 23 / 2013$ & 16.35 & 16 & 0.35 & 2.1875 \\
\hline Formate & $01 / 24 / 2013$ & 16.51 & 16 & 0.51 & 3.1875 \\
\hline Formate & $01 / 24 / 2013$ & 16.52 & 16 & 0.52 & 3.25 \\
\hline Formate & $01 / 25 / 2013$ & 16.48 & 16 & 0.48 & 3 \\
\hline Formate & $01 / 25 / 2013$ & 16.55 & 16 & 0.55 & 3.4375 \\
\hline Formate & $01 / 25 / 2013$ & 16.55 & 16 & 0.55 & 3.4375 \\
\hline Formate & $01 / 26 / 2013$ & 16.27 & 16 & 0.27 & 1.6875 \\
\hline Formate & $01 / 26 / 2013$ & 16.47 & 16 & 0.47 & 2.9375 \\
\hline Formate & $01 / 26 / 2013$ & 16.48 & 16 & 0.48 & 3 \\
\hline Formate & $01 / 26 / 2013$ & 16.52 & 16 & 0.52 & 3.25 \\
\hline Formate & $01 / 26 / 2013$ & 16.53 & 16 & 0.53 & 3.3125 \\
\hline Formate & $01 / 27 / 2013$ & 16.5 & 16 & 0.5 & 3.125 \\
\hline Formate & $01 / 27 / 2013$ & 16.55 & 16 & 0.55 & 3.4375 \\
\hline Formate & $01 / 28 / 2013$ & 15.79 & 16 & -0.21 & \begin{tabular}{|l}
-1.3125 \\
\end{tabular} \\
\hline Formate & $01 / 28 / 2013$ & 16.5 & 16 & 0.5 & 3.125 \\
\hline Formate & $01 / 28 / 2013$ & 16.5 & 16 & 0.5 & 3.125 \\
\hline Formate & $01 / 29 / 2013$ & 16.42 & 16 & 0.42 & 2.625 \\
\hline Formate & $01 / 29 / 2013$ & 16.44 & 16 & 0.44 & 2.75 \\
\hline Formate & 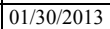 & 16.32 & 16 & 0.32 & 2 \\
\hline Formate & $01 / 31 / 2013$ & 15.96 & 16 & -0.04 & -0.25 \\
\hline Formate & $01 / 31 / 2013$ & 16.41 & 16 & 0.41 & 2.5625 \\
\hline Formate & 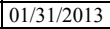 & 16.42 & 16 & 0.42 & 2.625 \\
\hline Formate & $02 / 01 / 2013$ & 15.78 & 16 & \begin{tabular}{|l|}
-0.22 \\
\end{tabular} & -1.375 \\
\hline Formate & \begin{tabular}{|l|l|}
$02 / 01 / 2013$ \\
\end{tabular} & 15.84 & 16 & -0.16 & -1 \\
\hline Formate & $02 / 01 / 2013$ & 16.47 & 16 & \begin{tabular}{|l|}
0.47 \\
\end{tabular} & 2.9375 \\
\hline Formate & $02 / 01 / 2013$ & 16.47 & 16 & 0.47 & 2.9375 \\
\hline Formate & $02 / 01 / 2013$ & 16.47 & 16 & 0.47 & 2.9375 \\
\hline Formate & $02 / 01 / 2013$ & 16.47 & 16 & 0.47 & 2.9375 \\
\hline Formate & $02 / 02 / 2013$ & 16.49 & 16 & 0.49 & 3.0625 \\
\hline Formate & \begin{tabular}{|l}
$02 / 03 / 2013$ \\
\end{tabular} & 16.46 & 16 & 0.46 & 2.875 \\
\hline Formate & $02 / 03 / 2013$ & 16.5 & 16 & 0.5 & 3.125 \\
\hline Formate & $02 / 03 / 2013$ & 16.5 & 16 & 0.5 & 3.125 \\
\hline Formate & $02 / 04 / 2013$ & 15.82 & 16 & -0.18 & -1.125 \\
\hline Formate & $02 / 04 / 2013$ & 16.45 & 16 & 0.45 & 2.8125 \\
\hline Formate & $02 / 05 / 2013$ & 15.24 & 16 & \begin{tabular}{|l|}
-0.76 \\
\end{tabular} & -4.75 \\
\hline Formate & 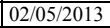 & 16.44 & 16 & \begin{tabular}{|l|}
0.44 \\
\end{tabular} & 2.75 \\
\hline Formate & $02 / 06 / 2013$ & 16.18 & 16 & 0.18 & 1.125 \\
\hline Formate & $02 / 07 / 2013$ & 16.1 & 16 & 0.1 & 0.625 \\
\hline Formate & 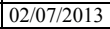 & 16.4 & 16 & 0.4 & 2.5 \\
\hline Formate & $02 / 08 / 2013$ & 16.41 & 16 & 0.41 & 2.5625 \\
\hline Formate & $02 / 09 / 2013$ & 16.42 & 16 & 0.42 & 2.625 \\
\hline Formate & \begin{tabular}{|l|l}
$02 / 09 / 2013$ \\
\end{tabular} & 16.42 & 16 & 0.42 & 2.625 \\
\hline Formate & 02/09/2013 & 16.43 & 16 & 0.43 & 2.6875 \\
\hline Formate & \begin{tabular}{|l|l|}
$02 / 10 / 2013$ \\
\end{tabular} & 16.41 & 16 & 0.41 & 2.5625 \\
\hline Nitrate & $11 / 09 / 2012$ & 2.02 & 2 & 0.02 & 1 \\
\hline Nitrate & $11 / 10 / 2012$ & 1.99 & 2 & -0.01 & -0.5 \\
\hline Nitrate & $11 / 11 / 2012$ & 1.98 & 2 & -0.02 & -1 \\
\hline Nitrate & $11 / 13 / 2012$ & 1.95 & 2 & -0.05 & -2.5 \\
\hline Nitrate & $11 / 13 / 2012$ & 1.96 & 2 & -0.04 & -2 \\
\hline Nitrate & $11 / 14 / 2012$ & 1.95 & 2 & -0.05 & -2.5 \\
\hline Nitrate & $11 / 16 / 2012$ & 1.95 & 2 & -0.05 & -2.5 \\
\hline Nitrate & $11 / 16 / 2012$ & 2 & 2 & 0 & 0 \\
\hline Nitrate & $11 / 17 / 2012$ & 1.93 & 2 & -0.07 & -3.5 \\
\hline Nitrate & $11 / 18 / 2012$ & 1.96 & 2 & \begin{tabular}{|l|}
-0.04 \\
\end{tabular} & -2 \\
\hline Nitrate & $11 / 19 / 2012$ & 1.97 & 2 & -0.03 & -1.5 \\
\hline Nitrate & $11 / 20 / 2012$ & 1.96 & 2 & -0.04 & -2 \\
\hline Nitrate & $11 / 21 / 2012$ & 1.86 & 2 & -0.14 & -7 \\
\hline Nitrate & $11 / 21 / 2012$ & 2 & 2 & 0 & 0 \\
\hline Nitrate & $11 / 21 / 2012$ & 2 & 2 & 0 & 0 \\
\hline Nitrate & $11 / 22 / 2012$ & 1.9 & 2 & -0.1 & -5 \\
\hline Nitrate & $11 / 24 / 2012$ & 1.88 & 2 & -0.12 & -6 \\
\hline Nitrate & $11 / 24 / 2012$ & 1.94 & 2 & $\mid-0.06$ & -3 \\
\hline Nitrate & $11 / 24 / 2012$ & 1.99 & 2 & -0.01 & -0.5 \\
\hline Nitrate & $11 / 24 / 2012$ & 2 & 2 & 0 & 0 \\
\hline Nitrate & $11 / 25 / 2012$ & 1.98 & 2 & -0.02 & -1 \\
\hline Nitrate & $11 / 25 / 2012$ & 2 & 2 & 0 & 0 \\
\hline Nitrate & $11 / 25 / 2012$ & 2 & 2 & 0 & 0 \\
\hline Nitrate & $11 / 25 / 2012$ & 2.11 & 2 & 0.11 & 5.5 \\
\hline Nitrate & $11 / 26 / 2012$ & 2.08 & 2 & 0.08 & 4 \\
\hline Nitrate & $11 / 26 / 2012$ & 2.1 & 2 & 0.1 & 5 \\
\hline Nitrate & $11 / 27 / 2012$ & 2.11 & 2 & 0.11 & 5.5 \\
\hline Nitrate & $11 / 28 / 2012$ & 2.07 & 2 & 0.07 & 3.5 \\
\hline Nitrate & $11 / 28 / 2012$ & 2.11 & 2 & 0.11 & 5.5 \\
\hline Nitrate & $11 / 28 / 2012$ & 2.11 & 2 & 0.11 & 5.5 \\
\hline
\end{tabular}


SRNL-STI-2013-00139

Revision 0

Table A1. DWPF Laboratory Measurements of Anion and TOC Standards

\begin{tabular}{|c|c|c|c|c|c|}
\hline Analyte & Date & $\begin{array}{c}\text { Measurement } \\
\text { (ppm) }\end{array}$ & $\begin{array}{c}\text { Reference } \\
\text { Value (ppm) }\end{array}$ & diff & $\begin{array}{l}\text { \% rel } \\
\text { diff }\end{array}$ \\
\hline Nitrate & $11 / 29 / 2012$ & 2.1 & 2 & 0.1 & 5 \\
\hline Nitrate & $11 / 30 / 2012$ & 2.02 & 2 & 0.02 & 1 \\
\hline Nitrate & $12 / 02 / 2012$ & 1.97 & 2 & $\mid-0.03$ & -1.5 \\
\hline Nitrate & $12 / 02 / 2012$ & 1.99 & 2 & -0.01 & -0.5 \\
\hline Nitrate & $12 / 03 / 2012$ & 1.99 & 2 & \begin{tabular}{|l|}
-0.01 \\
\end{tabular} & -0.5 \\
\hline Nitrate & $12 / 03 / 2012$ & 1.99 & 2 & $\mid-0.01$ & -0.5 \\
\hline Nitrate & $12 / 04 / 2012$ & 1.87 & 2 & \begin{tabular}{|l|}
-0.13 \\
\end{tabular} & -6.5 \\
\hline Nitrate & $12 / 06 / 2012$ & 1.99 & 2 & \begin{tabular}{|l|}
-0.01 \\
\end{tabular} & -0.5 \\
\hline Nitrate & $12 / 06 / 2012$ & 1.99 & 2 & -0.01 & -0.5 \\
\hline Nitrate & $12 / 07 / 2012$ & 1.89 & 2 & \begin{tabular}{|l|}
-0.11 \\
\end{tabular} & -5.5 \\
\hline Nitrate & $12 / 08 / 2012$ & 1.97 & 2 & -0.03 & -1.5 \\
\hline Nitrate & $12 / 09 / 2012$ & 1.81 & 2 & \begin{tabular}{|l|}
-0.19 \\
\end{tabular} & -9.5 \\
\hline Nitrate & $12 / 09 / 2012$ & 1.93 & 2 & \begin{tabular}{|l|}
-0.07 \\
\end{tabular} & -3.5 \\
\hline Nitrate & $12 / 09 / 2012$ & 1.96 & 2 & -0.04 & -2 \\
\hline Nitrate & $12 / 09 / 2012$ & 1.97 & 2 & \begin{tabular}{|l|}
-0.03 \\
\end{tabular} & -1.5 \\
\hline Nitrate & $12 / 09 / 2012$ & 1.98 & 2 & -0.02 & -1 \\
\hline Nitrate & 12/10/2012 & 1.97 & 2 & \begin{tabular}{|l|}
-0.03 \\
\end{tabular} & -1.5 \\
\hline Nitrate & $12 / 11 / 2012$ & 1.91 & 2 & \begin{tabular}{|l|}
-0.09 \\
\end{tabular} & -4.5 \\
\hline Nitrate & $12 / 12 / 2012$ & 1.87 & $2 \mid$ & -0.13 & -6.5 \\
\hline Nitrate & $12 / 12 / 2012$ & 1.93 & 2 & \begin{tabular}{|l|}
-0.07 \\
\end{tabular} & -3.5 \\
\hline Nitrate & $12 / 14 / 2012$ & 1.98 & 2 & \begin{tabular}{|l|}
-0.02 \\
\end{tabular} & -1 \\
\hline Nitrate & $12 / 14 / 2012$ & 1.98 & 2 & $\mid-0.02$ & -1 \\
\hline Nitrate & $12 / 15 / 2012$ & 1.93 & 2 & \begin{tabular}{|l|}
-0.07 \\
\end{tabular} & -3.5 \\
\hline Nitrate & $12 / 15 / 2012$ & 1.96 & 2 & \begin{tabular}{|l|l|}
-0.04 \\
\end{tabular} & -2 \\
\hline Nitrate & $12 / 15 / 2012$ & 1.96 & 2 & \begin{tabular}{|l|}
-0.04 \\
\end{tabular} & -2 \\
\hline Nitrate & $12 / 16 / 2012$ & 1.96 & 2 & \begin{tabular}{|l|}
-0.04 \\
\end{tabular} & -2 \\
\hline Nitrate & $12 / 17 / 2012$ & 1.83 & 2 & \begin{tabular}{|l|}
-0.17 \\
\end{tabular} & -8.5 \\
\hline Nitrate & $12 / 17 / 2012$ & 1.96 & 2 & \begin{tabular}{|l|}
-0.04 \\
\end{tabular} & -2 \\
\hline Nitrate & $12 / 17 / 2012$ & 1.96 & 2 & \begin{tabular}{|l|}
-0.04 \\
\end{tabular} & -2 \\
\hline Nitrate & $12 / 18 / 2012$ & 1.95 & 2 & -0.05 & -2.5 \\
\hline Nitrate & $12 / 20 / 2012$ & 1.98 & 2 & -0.02 & -1 \\
\hline Nitrate & $12 / 20 / 2012$ & 1.98 & 2 & \begin{tabular}{|l|}
-0.02 \\
\end{tabular} & -1 \\
\hline Nitrate & $12 / 21 / 2012$ & 1.95 & 2 & \begin{tabular}{|l|}
-0.05 \\
\end{tabular} & -2.5 \\
\hline Nitrate & $12 / 21 / 2012$ & 1.96 & 2 & -0.04 & -2 \\
\hline Nitrate & $12 / 22 / 2012$ & 1.97 & 2 & $\mid-0.03$ & -1.5 \\
\hline Nitrate & $12 / 23 / 2012$ & 1.98 & 2 & \begin{tabular}{|l|}
-0.02 \\
\end{tabular} & -1 \\
\hline Nitrate & $12 / 23 / 2012$ & 1.98 & 2 & $\mid-0.02$ & -1 \\
\hline Nitrate & $12 / 23 / 2012$ & 2 & 2 & 0 & 0 \\
\hline Nitrate & $12 / 23 / 2012$ & 2.03 & $2 \mid$ & \begin{tabular}{|l|}
0.03 \\
\end{tabular} & 1.5 \\
\hline Nitrate & $12 / 24 / 2012$ & 1.91 & 2 & \begin{tabular}{|l|l|}
-0.09 \\
\end{tabular} & -4.5 \\
\hline Nitrate & $12 / 24 / 2012$ & 1.99 & 2 & -0.01 & -0.5 \\
\hline Nitrate & $12 / 25 / 2012$ & 1.94 & 2 & \begin{tabular}{|l|}
-0.06 \\
\end{tabular} & -3 \\
\hline Nitrate & $12 / 25 / 2012$ & 1.94 & 2 & \begin{tabular}{|l|l|}
-0.06 \\
\end{tabular} & -3 \\
\hline Nitrate & $12 / 27 / 2012$ & 1.98 & 2 & \begin{tabular}{|l|}
-0.02 \\
\end{tabular} & -1 \\
\hline Nitrate & $12 / 27 / 2012$ & 1.98 & 2 & \begin{tabular}{|l|}
-0.02 \\
\end{tabular} & -1 \\
\hline Nitrate & $12 / 27 / 2012$ & 1.98 & 2 & \begin{tabular}{|l|}
-0.02 \\
\end{tabular} & -1 \\
\hline Nitrate & $12 / 28 / 2012$ & 1.84 & 2 & \begin{tabular}{|l|l|}
-0.16 \\
\end{tabular} & -8 \\
\hline Nitrate & $12 / 28 / 2012$ & 1.98 & 2 & -0.02 & -1 \\
\hline Nitrate & $12 / 28 / 2012$ & 1.98 & 2 & \begin{tabular}{|l|}
-0.02 \\
\end{tabular} & -1 \\
\hline Nitrate & $12 / 29 / 2012$ & 1.96 & 2 & \begin{tabular}{|l|}
-0.04 \\
\end{tabular} & -2 \\
\hline Nitrate & $12 / 29 / 2012$ & 1.96 & 2 & \begin{tabular}{|l|}
-0.04 \\
\end{tabular} & -2 \\
\hline Nitrate & $12 / 29 / 2012$ & 1.99 & 2 & \begin{tabular}{|l|}
-0.01 \\
\end{tabular} & -0.5 \\
\hline Nitrate & $12 / 30 / 2012$ & 1.98 & 2 & \begin{tabular}{|l|l|}
-0.02 \\
\end{tabular} & -1 \\
\hline Nitrate & $12 / 31 / 2012$ & 1.94 & 2 & -0.06 & -3 \\
\hline Nitrate & $12 / 31 / 2012$ & 1.98 & 2 & \begin{tabular}{|l|}
-0.02 \\
\end{tabular} & -1 \\
\hline Nitrate & $01 / 01 / 2013$ & 1.97 & 2 & \begin{tabular}{|l|}
-0.03 \\
\end{tabular} & -1.5 \\
\hline Nitrate & \begin{tabular}{|c|}
$01 / 01 / 2013$ \\
\end{tabular} & 1.98 & 2 & \begin{tabular}{|l|}
-0.02 \\
\end{tabular} & -1 \\
\hline Nitrate & $01 / 02 / 2013$ & 1.97 & 2 & \begin{tabular}{|l|}
-0.03 \\
\end{tabular} & -1.5 \\
\hline Nitrate & $01 / 03 / 2013$ & 1.94 & 2 & \begin{tabular}{|l|l|}
-0.06 \\
\end{tabular} & -3 \\
\hline Nitrate & $01 / 03 / 2013$ & 1.94 & 2 & \begin{tabular}{|l|}
-0.06 \\
\end{tabular} & -3 \\
\hline Nitrate & $01 / 04 / 2013$ & 1.92 & 2 & \begin{tabular}{|c|}
-0.08 \\
\end{tabular} & -4 \\
\hline Nitrate & $01 / 05 / 2013$ & 1.96 & 2 & \begin{tabular}{|l|}
-0.04 \\
\end{tabular} & -2 \\
\hline Nitrate & $\begin{array}{l}01 / 05 / 2013 \\
\end{array}$ & 1.97 & 2 & \begin{tabular}{|l|}
-0.03 \\
\end{tabular} & -1.5 \\
\hline Nitrate & $01 / 06 / 2013$ & 1.92 & 2 & -0.08 & -4 \\
\hline Nitrate & $01 / 07 / 2013$ & 1.77 & $2 \mid$ & \begin{tabular}{|l|}
-0.23 \\
\end{tabular} & -11.5 \\
\hline Nitrate & $01 / 08 / 2013$ & 1.9 & 2 & \begin{tabular}{|l|}
-0.1 \\
\end{tabular} & -5 \\
\hline Nitrate & $01 / 11 / 2013$ & 1.78 & 2 & \begin{tabular}{|l|}
-0.22 \\
\end{tabular} & -11 \\
\hline Nitrate & $01 / 11 / 2013$ & 1.96 & 2 & \begin{tabular}{|l|}
-0.04 \\
\end{tabular} & -2 \\
\hline Nitrate & $01 / 12 / 2013$ & 1.77 & 2 & \begin{tabular}{|l|}
-0.23 \\
\end{tabular} & -11.5 \\
\hline Nitrate & $01 / 12 / 2013$ & 1.9 & 2 & \begin{tabular}{|l|}
-0.1 \\
\end{tabular} & -5 \\
\hline Nitrate & $01 / 13 / 2013$ & 1.91 & 2 & \begin{tabular}{|l|}
-0.09 \\
\end{tabular} & -4.5 \\
\hline Nitrate & $01 / 13 / 2013$ & 1.92 & $2 \mid$ & \begin{tabular}{|l|}
-0.08 \\
\end{tabular} & -4 \\
\hline Nitrate & $01 / 13 / 2013$ & 1.94 & 2 & \begin{tabular}{|l|}
-0.06 \\
\end{tabular} & -3 \\
\hline Nitrate & $01 / 14 / 2013$ & 1.85 & 2 & -0.15 & -7.5 \\
\hline Nitrate & $01 / 14 / 2013$ & 1.85 & 2 & -0.15 & -7.5 \\
\hline Nitrate & $01 / 14 / 2013$ & 1.91 & 2 & \begin{tabular}{|l|}
-0.09 \\
\end{tabular} & -4.5 \\
\hline Nitrate & $01 / 14 / 2013$ & 1.93 & 2 & \begin{tabular}{|l|}
-0.07 \\
\end{tabular} & -3.5 \\
\hline Nitrate & $01 / 14 / 2013$ & 1.96 & $2 \mid$ & \begin{tabular}{|l|}
-0.04 \\
\end{tabular} & -2 \\
\hline Nitrate & $01 / 15 / 2013$ & 2.01 & 2 & \begin{tabular}{|l|}
0.01 \\
\end{tabular} & 0.5 \\
\hline Nitrate & $01 / 16 / 2013$ & 1.99 & 2 & \begin{tabular}{|l|}
-0.01 \\
\end{tabular} & -0.5 \\
\hline Nitrate & \begin{tabular}{|l|}
$01 / 17 / 2013$ \\
\end{tabular} & 1.99 & $2 \mid$ & \begin{tabular}{|l|}
-0.01 \\
\end{tabular} & -0.5 \\
\hline Nitrate & \begin{tabular}{|l|}
$01 / 18 / 2013$ \\
\end{tabular} & 2.01 & 2 & \begin{tabular}{|l|}
0.01 \\
\end{tabular} & 0.5 \\
\hline Nitrate & $01 / 18 / 2013$ & 2.01 & 2 & 0.01 & 0.5 \\
\hline
\end{tabular}

\begin{tabular}{|c|c|c|c|c|c|}
\hline Analyte & Date & $\begin{array}{c}\text { Measurement } \\
\text { (ppm) }\end{array}$ & $\begin{array}{c}\text { Reference } \\
\text { Value (ppm) }\end{array}$ & diff & $\begin{array}{l}\text { \% rel } \\
\text { diff }\end{array}$ \\
\hline Nitrate & \begin{tabular}{|l|}
$01 / 18 / 2013$ \\
\end{tabular} & 2.08 & 2 & 0.08 & 4 \\
\hline Nitrate & $01 / 18 / 2013$ & 2.08 & 2 & 0.08 & 4 \\
\hline Nitrate & $01 / 18 / 2013$ & 2.1 & 2 & 0.1 & 5 \\
\hline Nitrate & $01 / 19 / 2013$ & 2.02 & 2 & 0.02 & 1 \\
\hline Nitrate & $01 / 19 / 2013$ & 2.08 & 2 & 0.08 & 4 \\
\hline Nitrate & $01 / 19 / 2013$ & 2.08 & 2 & 0.08 & 4 \\
\hline Nitrate & $01 / 20 / 2013$ & 2.05 & 2 & 0.05 & 2.5 \\
\hline Nitrate & $01 / 21 / 2013$ & 2.03 & 2 & 0.03 & 1.5 \\
\hline Nitrate & $01 / 21 / 2013$ & 2.08 & 2 & 0.08 & 4 \\
\hline Nitrate & $01 / 21 / 2013$ & 2.09 & 2 & 0.09 & 4.5 \\
\hline Nitrate & $01 / 22 / 2013$ & 2.02 & 2 & 0.02 & 1 \\
\hline Nitrate & $01 / 22 / 2013$ & 2.04 & 2 & 0.04 & 2 \\
\hline Nitrate & $01 / 22 / 2013$ & 2.08 & 2 & 0.08 & 4 \\
\hline Nitrate & $01 / 23 / 2013$ & 2.03 & 2 & 0.03 & 1.5 \\
\hline Nitrate & $01 / 23 / 2013$ & 2.03 & 2 & 0.03 & 1.5 \\
\hline Nitrate & $01 / 23 / 2013$ & 2.04 & 2 & 0.04 & 2 \\
\hline Nitrate & $01 / 23 / 2013$ & 2.05 & 2 & 0.05 & 2.5 \\
\hline Nitrate & $01 / 24 / 2013$ & 2.08 & 2 & 0.08 & 4 \\
\hline Nitrate & $01 / 24 / 2013$ & 2.09 & 2 & 0.09 & 4.5 \\
\hline Nitrate & $01 / 25 / 2013$ & 2.04 & 2 & 0.04 & 2 \\
\hline Nitrate & $01 / 25 / 2013$ & 2.06 & 2 & 0.06 & 3 \\
\hline Nitrate & $01 / 25 / 2013$ & 2.08 & 2 & 0.08 & 4 \\
\hline Nitrate & $01 / 26 / 2013$ & 2.07 & 2 & 0.07 & 3.5 \\
\hline Nitrate & $01 / 26 / 2013$ & 2.07 & 2 & 0.07 & 3.5 \\
\hline Nitrate & $01 / 26 / 2013$ & 2.07 & 2 & 0.07 & 3.5 \\
\hline Nitrate & $01 / 26 / 2013$ & 2.08 & 2 & 0.08 & 4 \\
\hline Nitrate & $01 / 26 / 2013$ & 2.08 & 2 & 0.08 & 4 \\
\hline Nitrate & $01 / 27 / 2013$ & 2.07 & 2 & 0.07 & 3.5 \\
\hline Nitrate & $01 / 27 / 2013$ & 2.07 & 2 & 0.07 & 3.5 \\
\hline Nitrate & $01 / 28 / 2013$ & 1.96 & 2 & \begin{tabular}{|l|}
-0.04 \\
\end{tabular} & -2 \\
\hline Nitrate & $01 / 28 / 2013$ & 2.07 & 2 & \begin{tabular}{|l|}
0.07 \\
\end{tabular} & 3.5 \\
\hline Nitrate & $01 / 28 / 2013$ & 2.07 & 2 & 0.07 & 3.5 \\
\hline Nitrate & \begin{tabular}{|l|}
$01 / 29 / 2013$ \\
\end{tabular} & 2.04 & 2 & 0.04 & 2 \\
\hline Nitrate & $01 / 29 / 2013$ & 2.07 & 2 & 0.07 & 3.5 \\
\hline Nitrate & $01 / 30 / 2013$ & 2.05 & 2 & 0.05 & 2.5 \\
\hline Nitrate & $01 / 31 / 2013$ & 2.05 & 2 & 0.05 & 2.5 \\
\hline Nitrate & $01 / 31 / 2013$ & 2.07 & 2 & 0.07 & 3.5 \\
\hline Nitrate & $02 / 01 / 2013$ & 1.95 & 2 & -0.05 & -2.5 \\
\hline Nitrate & $02 / 01 / 2013$ & 1.99 & 2 & -0.01 & -0.5 \\
\hline Nitrate & \begin{tabular}{|l|}
$02 / 01 / 2013$ \\
\end{tabular} & 2.02 & 2 & 0.02 & 1 \\
\hline Nitrate & $02 / 01 / 2013$ & 2.02 & 2 & 0.02 & 1 \\
\hline Nitrate & $02 / 01 / 2013$ & 2.05 & 2 & 0.05 & 2.5 \\
\hline Nitrate & \begin{tabular}{|l|}
$02 / 01 / 2013$ \\
\end{tabular} & 2.05 & 2 & 0.05 & 2.5 \\
\hline Nitrate & \begin{tabular}{|l|}
$02 / 02 / 2013$ \\
\end{tabular} & 2.07 & 2 & 0.07 & 3.5 \\
\hline Nitrate & $02 / 03 / 2013$ & 2.01 & 2 & 0.01 & 0.5 \\
\hline Nitrate & \begin{tabular}{|l|}
$02 / 03 / 2013$ \\
\end{tabular} & 2.01 & 2 & 0.01 & 0.5 \\
\hline Nitrate & $02 / 03 / 2013$ & 2.07 & 2 & 0.07 & 3.5 \\
\hline Nitrate & $02 / 04 / 2013$ & 1.97 & 2 & \begin{tabular}{|l|}
-0.03 \\
\end{tabular} & -1.5 \\
\hline Nitrate & \begin{tabular}{|l|}
$02 / 04 / 2013$ \\
\end{tabular} & 2.02 & 2 & \begin{tabular}{|l|}
0.02 \\
\end{tabular} & 1 \\
\hline Nitrate & $02 / 05 / 2013$ & 1.99 & 2 & -0.01 & -0.5 \\
\hline Nitrate & $02 / 05 / 2013$ & 2 & 2 & 0 & 0 \\
\hline Nitrate & $02 / 06 / 2013$ & 2.02 & 2 & 0.02 & 1 \\
\hline Nitrate & \begin{tabular}{|l|}
$02 / 07 / 2013$ \\
\end{tabular} & 2.03 & 2 & 0.03 & 1.5 \\
\hline Nitrate & $02 / 07 / 2013$ & 2.05 & 2 & 0.05 & 2.5 \\
\hline Nitrate & \begin{tabular}{|l|}
$02 / 08 / 2013$ \\
\end{tabular} & 2.04 & 2 & 0.04 & 2 \\
\hline Nitrate & \begin{tabular}{|l|}
$02 / 09 / 2013$ \\
\end{tabular} & 2.04 & 2 & 0.04 & 2 \\
\hline Nitrate & \begin{tabular}{|l|}
$02 / 09 / 2013$ \\
\end{tabular} & 2.04 & 2 & 0.04 & 2 \\
\hline Nitrate & \begin{tabular}{|l|}
$02 / 09 / 2013$ \\
\end{tabular} & 2.06 & 2 & 0.06 & 3 \\
\hline Nitrate & \begin{tabular}{|l|}
$02 / 10 / 2013$ \\
\end{tabular} & 2.06 & 2 & 0.06 & 3 \\
\hline Nitrate & \begin{tabular}{|l|}
$11 / 09 / 2012$ \\
\end{tabular} & 16.5 & 16 & 0.5 & 3.125 \\
\hline Nitrate & $11 / 10 / 2012$ & 16.21 & 16 & 0.21 & 1.3125 \\
\hline Nitrate & $11 / 11 / 2012$ & 16.12 & 16 & 0.12 & 0.75 \\
\hline Nitrate & $11 / 13 / 2012$ & 16.01 & 16 & 0.01 & 0.0625 \\
\hline Nitrate & \begin{tabular}{|l|}
$11 / 13 / 2012$ \\
\end{tabular} & 16.03 & 16 & 0.03 & 0.1875 \\
\hline Nitrate & $11 / 14 / 2012$ & 15.95 & 16 & -0.05 & -0.3125 \\
\hline Nitrate & \begin{tabular}{|l|}
$11 / 16 / 2012$ \\
\end{tabular} & 16.17 & 16 & 0.17 & 1.0625 \\
\hline Nitrate & $11 / 16 / 2012$ & 16.44 & 16 & 0.44 & 2.75 \\
\hline Nitrate & $11 / 17 / 2012$ & 16.36 & 16 & 0.36 & 2.25 \\
\hline Nitrate & $11 / 18 / 2012$ & 16.18 & 16 & 0.18 & 1.125 \\
\hline Nitrate & $11 / 19 / 2012$ & 16.24 & 16 & 0.24 & 1.5 \\
\hline Nitrate & $11 / 20 / 2012$ & 16.04 & 16 & 0.04 & 0.25 \\
\hline Nitrate & $11 / 21 / 2012$ & 15 & 16 & -1 & -6.25 \\
\hline Nitrate & \begin{tabular}{|l|}
$11 / 21 / 2012$ \\
\end{tabular} & 16.12 & 16 & 0.12 & 0.75 \\
\hline Nitrate & $11 / 21 / 2012$ & 16.28 & 16 & \begin{tabular}{|l|}
0.28 \\
\end{tabular} & 1.75 \\
\hline Nitrate & $11 / 22 / 2012$ & 15.49 & 16 & -0.51 & -3.1875 \\
\hline Nitrate & $11 / 24 / 2012$ & 15.57 & 16 & \begin{tabular}{|l|}
-0.43 \\
\end{tabular} & -2.6875 \\
\hline Nitrate & $11 / 24 / 2012$ & 16.12 & 16 & 0.12 & 0.75 \\
\hline Nitrate & $11 / 24 / 2012$ & 16.25 & 16 & 0.25 & 1.5625 \\
\hline Nitrate & \begin{tabular}{|l|}
$11 / 24 / 2012$ \\
\end{tabular} & 16.3 & 16 & 0.3 & 1.875 \\
\hline Nitrate & $11 / 25 / 2012$ & 15.84 & 16 & -0.16 & -1 \\
\hline Nitrate & $11 / 25 / 2012$ & 15.84 & 16 & -0.16 & -1 \\
\hline Nitrate & $11 / 25 / 2012$ & 16.3 & 16 & \begin{tabular}{|l|}
0.3 \\
\end{tabular} & 1.875 \\
\hline Nitrate & $11 / 25 / 2012$ & 16.39 & 16 & 0.39 & 2.4375 \\
\hline
\end{tabular}


SRNL-STI-2013-00139

Revision 0

Table A1. DWPF Laboratory Measurements of Anion and TOC Standards

\begin{tabular}{|c|c|c|c|c|c|}
\hline Analyte & Date & $\begin{array}{c}\text { Measurement } \\
\text { (ppm) }\end{array}$ & $\begin{array}{c}\text { Reference } \\
\text { Value (ppm) }\end{array}$ & diff & $\begin{array}{l}\text { \% rel } \\
\text { diff }\end{array}$ \\
\hline Nitrate & $11 / 26 / 2012$ & 15.61 & 16 & \begin{tabular}{|l|}
-0.39 \\
\end{tabular} & -2.4375 \\
\hline Nitrate & $11 / 26 / 2012$ & 16.01 & 16 & 0.01 & 0.0625 \\
\hline Nitrate & $11 / 27 / 2012$ & 16.35 & 16 & 0.35 & 2.1875 \\
\hline Nitrate & $11 / 28 / 2012$ & 16.32 & 16 & 0.32 & 2 \\
\hline Nitrate & $11 / 28 / 2012$ & 16.37 & 16 & 0.37 & 2.3125 \\
\hline Nitrate & $11 / 28 / 2012$ & 16.37 & 16 & 0.37 & 2.3125 \\
\hline Nitrate & $11 / 29 / 2012$ & 16.38 & 16 & 0.38 & 2.375 \\
\hline Nitrate & $11 / 30 / 2012$ & 15.7 & 16 & -0.3 & -1.875 \\
\hline Nitrate & $12 / 02 / 2012$ & 15.94 & 16 & -0.06 & -0.375 \\
\hline Nitrate & $12 / 02 / 2012$ & 16.07 & 16 & \begin{tabular}{|l|}
0.07 \\
\end{tabular} & 0.4375 \\
\hline Nitrate & $12 / 03 / 2012$ & 15.65 & 16 & \begin{tabular}{|l|}
-0.35 \\
\end{tabular} & -2.1875 \\
\hline Nitrate & $12 / 03 / 2012$ & 16.23 & 16 & 0.23 & 1.4375 \\
\hline Nitrate & $12 / 04 / 2012$ & 15.94 & 16 & \begin{tabular}{|l|}
-0.06 \\
\end{tabular} & -0.375 \\
\hline Nitrate & $12 / 06 / 2012$ & 16.24 & 16 & 0.24 & 1.5 \\
\hline Nitrate & $12 / 06 / 2012$ & 16.24 & 16 & 0.24 & 1.5 \\
\hline Nitrate & $12 / 07 / 2012$ & 14.74 & 16 & -1.26 & -7.875 \\
\hline Nitrate & $12 / 08 / 2012$ & 15.79 & 16 & \begin{tabular}{|l|}
-0.21 \\
\end{tabular} & -1.3125 \\
\hline Nitrate & $12 / 09 / 2012$ & 14.95 & 16 & \begin{tabular}{|c|}
-1.05 \\
\end{tabular} & -6.5625 \\
\hline Nitrate & $12 / 09 / 2012$ & 15.7 & 16 & \begin{tabular}{|l|}
-0.3 \\
\end{tabular} & -1.875 \\
\hline Nitrate & $12 / 09 / 2012$ & 15.91 & 16 & \begin{tabular}{|c|}
-0.09 \\
\end{tabular} & -0.5625 \\
\hline Nitrate & $12 / 09 / 2012$ & 16.19 & 16 & \begin{tabular}{|l|}
0.19 \\
\end{tabular} & 1.1875 \\
\hline Nitrate & $12 / 09 / 2012$ & 16.26 & 16 & 0.26 & 1.625 \\
\hline Nitrate & $12 / 10 / 2012$ & 16.24 & 16 & 0.24 & 1.5 \\
\hline Nitrate & $12 / 11 / 2012$ & 15.7 & 16 & -0.3 & -1.875 \\
\hline Nitrate & $12 / 12 / 2012$ & 15 & 16 & -1 & -6.25 \\
\hline Nitrate & $12 / 12 / 2012$ & 15.47 & 16 & \begin{tabular}{|l|}
-0.53 \\
\end{tabular} & -3.3125 \\
\hline Nitrate & $12 / 14 / 2012$ & 16.28 & 16 & 0.28 & 1.75 \\
\hline Nitrate & $12 / 14 / 2012$ & 16.33 & 16 & 0.33 & 2.0625 \\
\hline Nitrate & $12 / 15 / 2012$ & 15.99 & 16 & \begin{tabular}{|l|}
-0.01 \\
\end{tabular} & -0.0625 \\
\hline Nitrate & $12 / 15 / 2012$ & 16.12 & 16 & \begin{tabular}{|l|}
0.12 \\
\end{tabular} & 0.75 \\
\hline Nitrate & $12 / 15 / 2012$ & 16.12 & 16 & 0.12 & 0.75 \\
\hline Nitrate & $12 / 16 / 2012$ & 16.1 & 16 & 0.1 & 0.625 \\
\hline Nitrate & $12 / 17 / 2012$ & 16.07 & 16 & 0.07 & 0.4375 \\
\hline Nitrate & $12 / 17 / 2012$ & 16.08 & 16 & 0.08 & 0.5 \\
\hline Nitrate & $12 / 17 / 2012$ & 16.11 & 16 & 0.11 & 0.6875 \\
\hline Nitrate & $12 / 18 / 2012$ & 16.11 & 16 & \begin{tabular}{|l|}
0.11 \\
\end{tabular} & 0.6875 \\
\hline Nitrate & $12 / 20 / 2012$ & 15.89 & 16 & \begin{tabular}{|l|}
-0.11 \\
\end{tabular} & -0.6875 \\
\hline Nitrate & $12 / 20 / 2012$ & 15.92 & 16 & \begin{tabular}{|c|}
-0.08 \\
\end{tabular} & -0.5 \\
\hline Nitrate & $12 / 21 / 2012$ & 16.21 & 16 & \begin{tabular}{|l|}
0.21 \\
\end{tabular} & 1.3125 \\
\hline Nitrate & $12 / 21 / 2012$ & 16.23 & 16 & 0.23 & 1.4375 \\
\hline Nitrate & $12 / 22 / 2012$ & 16.23 & 16 & 0.23 & 1.4375 \\
\hline Nitrate & $12 / 23 / 2012$ & 15.67 & 16 & \begin{tabular}{|l|}
-0.33 \\
\end{tabular} & -2.0625 \\
\hline Nitrate & $12 / 23 / 2012$ & 16.2 & 16 & \begin{tabular}{|l|}
0.2 \\
\end{tabular} & 1.25 \\
\hline Nitrate & $12 / 23 / 2012$ & 16.2 & 16 & 0.2 & 1.25 \\
\hline Nitrate & $12 / 23 / 2012$ & 16.26 & 16 & 0.26 & 1.625 \\
\hline Nitrate & $12 / 24 / 2012$ & 16.21 & 16 & 0.21 & 1.3125 \\
\hline Nitrate & $12 / 24 / 2012$ & 16.21 & 16 & 0.21 & 1.3125 \\
\hline Nitrate & $12 / 25 / 2012$ & 15.93 & 16 & \begin{tabular}{|c|}
-0.07 \\
\end{tabular} & -0.4375 \\
\hline Nitrate & $12 / 25 / 2012$ & 15.93 & 16 & \begin{tabular}{|l|}
-0.07 \\
\end{tabular} & -0.4375 \\
\hline Nitrate & $12 / 27 / 2012$ & 16.26 & 16 & \begin{tabular}{|l|}
0.26 \\
\end{tabular} & 1.625 \\
\hline Nitrate & $12 / 27 / 2012$ & 16.32 & 16 & 0.32 & 2 \\
\hline Nitrate & $12 / 27 / 2012$ & 16.32 & 16 & 0.32 & 2 \\
\hline Nitrate & $12 / 28 / 2012$ & 15.82 & 16 & \begin{tabular}{|l|}
-0.18 \\
\end{tabular} & -1.125 \\
\hline Nitrate & $12 / 28 / 2012$ & 16.28 & 16 & \begin{tabular}{|l|}
0.28 \\
\end{tabular} & 1.75 \\
\hline Nitrate & $12 / 28 / 2012$ & 16.28 & 16 & 0.28 & 1.75 \\
\hline Nitrate & $12 / 29 / 2012$ & 16.08 & 16 & 0.08 & 0.5 \\
\hline Nitrate & $12 / 29 / 2012$ & 16.17 & 16 & 0.17 & 1.0625 \\
\hline Nitrate & $12 / 29 / 2012$ & 16.17 & 16 & 0.17 & 1.0625 \\
\hline Nitrate & $12 / 30 / 2012$ & 16.29 & 16 & 0.29 & 1.8125 \\
\hline Nitrate & $12 / 31 / 2012$ & 16.25 & 16 & 0.25 & 1.5625 \\
\hline Nitrate & $12 / 31 / 2012$ & 16.27 & 16 & 0.27 & 1.6875 \\
\hline Nitrate & $01 / 01 / 2013$ & 16.12 & 16 & 0.12 & 0.75 \\
\hline Nitrate & $01 / 01 / 2013$ & 16.29 & 16 & \begin{tabular}{|l|}
0.29 \\
\end{tabular} & 1.8125 \\
\hline Nitrate & $01 / 02 / 2013$ & 16.1 & 16 & \begin{tabular}{|l|}
0.1 \\
\end{tabular} & 0.625 \\
\hline Nitrate & $01 / 03 / 2013$ & 16.11 & 16 & 0.11 & 0.6875 \\
\hline Nitrate & $01 / 03 / 2013$ & 16.24 & 16 & \begin{tabular}{|l|}
0.24 \\
\end{tabular} & 1.5 \\
\hline Nitrate & $01 / 04 / 2013$ & 15.86 & 16 & \begin{tabular}{|l|}
-0.14 \\
\end{tabular} & -0.875 \\
\hline Nitrate & $01 / 05 / 2013$ & 15.85 & 16 & \begin{tabular}{|c|}
-0.15 \\
\end{tabular} & -0.9375 \\
\hline Nitrate & $01 / 05 / 2013$ & 16.15 & 16 & \begin{tabular}{|l|}
0.15 \\
\end{tabular} & 0.9375 \\
\hline Nitrate & \begin{tabular}{|l|}
$01 / 06 / 2013$ \\
\end{tabular} & 15.68 & 16 & \begin{tabular}{|l|}
-0.32 \\
\end{tabular} & -2 \\
\hline Nitrate & $01 / 07 / 2013$ & 15.65 & 16 & \begin{tabular}{|c|}
-0.35 \\
\end{tabular} & -2.1875 \\
\hline Nitrate & $01 / 08 / 2013$ & 15.8 & 16 & \begin{tabular}{|l|}
-0.2 \\
\end{tabular} & -1.25 \\
\hline Nitrate & $01 / 11 / 2013$ & 15.02 & 16 & \begin{tabular}{|l|}
-0.98 \\
\end{tabular} & -6.125 \\
\hline Nitrate & $01 / 11 / 2013$ & 16.17 & 16 & \begin{tabular}{|l|}
0.17 \\
\end{tabular} & 1.0625 \\
\hline Nitrate & $01 / 12 / 2013$ & 14.82 & 16 & -1.18 & -7.375 \\
\hline Nitrate & $01 / 12 / 2013$ & 15.14 & 16 & \begin{tabular}{|l|}
-0.86 \\
\end{tabular} & -5.375 \\
\hline Nitrate & $01 / 13 / 2013$ & 14.75 & 16 & \begin{tabular}{|l|}
-1.25 \\
\end{tabular} & -7.8125 \\
\hline Nitrate & $01 / 13 / 2013$ & 14.98 & 16 & \begin{tabular}{|l|}
-1.02 \\
\end{tabular} & -6.375 \\
\hline Nitrate & $01 / 13 / 2013$ & 15.93 & 16 & \begin{tabular}{|l|}
-0.07 \\
\end{tabular} & -0.4375 \\
\hline Nitrate & $01 / 14 / 2013$ & 14.96 & 16 & \begin{tabular}{|l|}
-1.04 \\
\end{tabular} & -6.5 \\
\hline Nitrate & \begin{tabular}{|l|}
$01 / 14 / 2013$ \\
\end{tabular} & 14.96 & 16 & -1.04 & -6.5 \\
\hline Nitrate & \begin{tabular}{|l|}
$01 / 14 / 2013$ \\
\end{tabular} & 15.06 & 16 & \begin{tabular}{|l|}
-0.94 \\
\end{tabular} & -5.875 \\
\hline Nitrate & $01 / 14 / 2013$ & 15.26 & 16 & \begin{tabular}{|l|}
-0.74 \\
\end{tabular} & -4.625 \\
\hline
\end{tabular}

\begin{tabular}{|c|c|c|c|c|c|}
\hline Analyte & Date & \begin{tabular}{|c|}
$\begin{array}{c}\text { Measurement } \\
\text { (ppm) }\end{array}$ \\
\end{tabular} & $\begin{array}{c}\text { Reference } \\
\text { Value (ppm) }\end{array}$ & diff & $\begin{array}{c}\text { \% rel } \\
\text { diff }\end{array}$ \\
\hline Nitrate & $01 / 14 / 2013$ & 15.35 & 16 & -0.65 & -4.0625 \\
\hline Nitrate & $01 / 15 / 2013$ & 15.32 & 16 & $\mid-0.68$ & -4.25 \\
\hline Nitrate & $01 / 16 / 2013$ & 15.9 & 16 & $\mid-0.1$ & -0.625 \\
\hline Nitrate & $01 / 17 / 2013$ & 15.9 & 16 & -0.1 & -0.625 \\
\hline Nitrate & $01 / 18 / 2013$ & 15.22 & 16 & -0.78 & -4.875 \\
\hline Nitrate & $01 / 18 / 2013$ & 15.22 & 16 & \begin{tabular}{|l|} 
\\
\end{tabular} & -4.875 \\
\hline Nitrate & $01 / 18 / 2013$ & 15.9 & 16 & \begin{tabular}{|l|} 
\\
\end{tabular} & -0.625 \\
\hline Nitrate & $01 / 18 / 2013$ & 15.9 & 16 & -0.1 & -0.625 \\
\hline Nitrate & $01 / 18 / 2013$ & 16.17 & 16 & 0.17 & 1.0625 \\
\hline Nitrate & 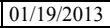 & 16.1 & 16 & 0.1 & 0.625 \\
\hline Nitrate & $01 / 19 / 2013$ & 16.2 & 16 & 0.2 & 1.25 \\
\hline Nitrate & \begin{tabular}{|l|l|l}
$01 / 2013$ \\
\end{tabular} & 16.23 & 16 & 0.23 & 1.4375 \\
\hline Nitrate & \begin{tabular}{|l}
$01 / 20 / 2013$ \\
\end{tabular} & 15.94 & 16 & -0.06 & -0.375 \\
\hline Nitrate & $01 / 21 / 2013$ & 15.83 & 16 & \begin{tabular}{|l|}
-0.17 \\
\end{tabular} & \begin{tabular}{|l|}
-1.0625 \\
\end{tabular} \\
\hline Nitrate & $01 / 21 / 2013$ & 15.83 & 16 & \begin{tabular}{|l|}
-0.17 \\
\end{tabular} & \begin{tabular}{|l}
-1.0625 \\
\end{tabular} \\
\hline Nitrate & $01 / 21 / 2013$ & 15.97 & 16 & -0.03 & \begin{tabular}{|l}
-0.1875 \\
\end{tabular} \\
\hline Nitrate & $01 / 22 / 2013$ & 15.96 & 16 & -0.04 & $\begin{array}{l}-0.25 \\
\end{array}$ \\
\hline Nitrate & $01 / 22 / 2013$ & 16.02 & 16 & \begin{tabular}{|l|}
0.02 \\
\end{tabular} & 0.125 \\
\hline Nitrate & $01 / 22 / 2013$ & 16.03 & 16 & 0.03 & 0.1875 \\
\hline Nitrate & $01 / 23 / 2013$ & 15.84 & 16 & -0.16 & -1 \\
\hline Nitrate & $01 / 23 / 2013$ & 15.92 & 16 & \begin{tabular}{|l|}
-0.08 \\
\end{tabular} & -0.5 \\
\hline Nitrate & $01 / 23 / 2013$ & 16.06 & 16 & \begin{tabular}{|l|}
0.06 \\
\end{tabular} & 0.375 \\
\hline Nitrate & $01 / 23 / 2013$ & 16.06 & 16 & 0.06 & 0.375 \\
\hline Nitrate & $01 / 24 / 2013$ & 16.32 & 16 & 0.32 & 2 \\
\hline Nitrate & $01 / 24 / 2013$ & 16.34 & 16 & 0.34 & 2.125 \\
\hline Nitrate & $01 / 25 / 2013$ & 16.23 & 16 & 0.23 & 1.4375 \\
\hline Nitrate & $01 / 25 / 2013$ & 16.27 & 16 & 0.27 & 1.6875 \\
\hline Nitrate & $01 / 25 / 2013$ & 16.3 & 16 & $\begin{array}{l}0.3 \\
\end{array}$ & 1.875 \\
\hline Nitrate & $01 / 26 / 2013$ & 16.11 & 16 & 0.11 & 0.6875 \\
\hline Nitrate & $01 / 26 / 2013$ & 16.27 & 16 & 0.27 & 1.6875 \\
\hline Nitrate & 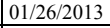 & 16.3 & 16 & 0.3 & 1.875 \\
\hline Nitrate & $01 / 26 / 2013$ & 16.31 & 16 & 0.31 & 1.9375 \\
\hline Nitrate & \begin{tabular}{|l|l|}
$01 / 26 / 2013$ \\
\end{tabular} & 16.33 & 16 & 0.33 & 2.0625 \\
\hline Nitrate & $01 / 27 / 2013$ & 16.34 & 16 & 0.34 & 2.125 \\
\hline Nitrate & $01 / 27 / 2013$ & 16.34 & 16 & 0.34 & 2.125 \\
\hline Nitrate & $01 / 28 / 2013$ & 15.59 & 16 & -0.41 & \begin{tabular}{|l}
-2.5625 \\
\end{tabular} \\
\hline Nitrate & $01 / 28 / 2013$ & 16.28 & 16 & 0.28 & $\begin{array}{l}1.75 \\
\end{array}$ \\
\hline Nitrate & $01 / 28 / 2013$ & 16.28 & 16 & 0.28 & 1.75 \\
\hline Nitrate & $01 / 29 / 2013$ & 16.23 & 16 & 0.23 & 1.4375 \\
\hline Nitrate & $01 / 29 / 2013$ & 16.24 & 16 & 0.24 & 1.5 \\
\hline Nitrate & $01 / 30 / 2013$ & 16.31 & 16 & 0.31 & 1.9375 \\
\hline Nitrate & $01 / 31 / 2013$ & 15.75 & 16 & -0.25 & \begin{tabular}{|l}
-1.5625 \\
\end{tabular} \\
\hline Nitrate & $01 / 31 / 2013$ & 16.25 & 16 & 0.25 & 1.5625 \\
\hline Nitrate & $02 / 01 / 2013$ & 15.64 & 16 & -0.36 & -2.25 \\
\hline Nitrate & 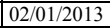 & 15.66 & 16 & \begin{tabular}{|l|}
-0.34 \\
\end{tabular} & -2.125 \\
\hline Nitrate & $02 / 01 / 2013$ & 16.31 & 16 & 0.31 & 1.9375 \\
\hline Nitrate & $02 / 01 / 2013$ & 16.31 & 16 & 0.31 & 1.9375 \\
\hline Nitrate & $02 / 01 / 2013$ & 16.31 & 16 & 0.31 & 1.9375 \\
\hline Nitrate & $02 / 01 / 2013$ & 16.31 & 16 & 0.31 & 1.9375 \\
\hline Nitrate & \begin{tabular}{|l|l|}
$02 / 02 / 2013$ \\
\end{tabular} & 16.27 & 16 & \begin{tabular}{|l|}
0.27 \\
\end{tabular} & 1.6875 \\
\hline Nitrate & $02 / 03 / 2013$ & 16.3 & 16 & \begin{tabular}{|l|}
0.3 \\
\end{tabular} & 1.875 \\
\hline Nitrate & $02 / 03 / 2013$ & 16.3 & 16 & 0.3 & 1.875 \\
\hline Nitrate & \begin{tabular}{|l|l|}
$02 / 03 / 2013$ \\
\end{tabular} & 16.3 & 16 & 0.3 & 1.875 \\
\hline Nitrate & $02 / 04 / 2013$ & 15.65 & 16 & \begin{tabular}{|l|}
-0.35 \\
\end{tabular} & $\begin{array}{l}-2.1875 \\
\end{array}$ \\
\hline Nitrate & $02 / 04 / 2013$ & 16.28 & 16 & 0.28 & 1.75 \\
\hline Nitrate & $02 / 05 / 2013$ & 15.12 & 16 & -0.88 & -5.5 \\
\hline Nitrate & $02 / 05 / 2013$ & 16.21 & 16 & 0.21 & 1.3125 \\
\hline Nitrate & $02 / 06 / 2013$ & 15.95 & 16 & -0.05 & \begin{tabular}{|l}
-0.3125 \\
\end{tabular} \\
\hline Nitrate & $02 / 07 / 2013$ & 15.93 & 16 & -0.07 & -0.4375 \\
\hline Nitrate & \begin{tabular}{|l|l|l|}
$02 / 013$ \\
\end{tabular} & 16.16 & 16 & 0.16 & $\begin{array}{l} \\
\end{array}$ \\
\hline Nitrate & $02 / 08 / 2013$ & 16.19 & 16 & 0.19 & 1.1875 \\
\hline Nitrate & $02 / 09 / 2013$ & 16.08 & 16 & 0.08 & 0.5 \\
\hline Nitrate & \begin{tabular}{|l|l|}
$02 / 09 / 2013$ \\
\end{tabular} & 16.15 & 16 & 0.15 & 0.9375 \\
\hline Nitrate & \begin{tabular}{|l|}
$02 / 09 / 2013$ \\
\end{tabular} & 16.18 & 16 & 0.18 & 1.125 \\
\hline Nitrate & $02 / 10 / 2013$ & 16.25 & 16 & 0.25 & 1.5625 \\
\hline Soluble Oxalate & $11 / 09 / 2012$ & 2.14 & 2 & 0.14 & 7 \\
\hline Soluble Oxalate & $11 / 10 / 2012$ & 2.11 & 2 & 0.11 & 5.5 \\
\hline Soluble Oxalate & $11 / 11 / 2012$ & 2.09 & 2 & 0.09 & 4.5 \\
\hline Soluble Oxalate & $11 / 13 / 2012$ & 2.05 & 2 & 0.05 & 2.5 \\
\hline Soluble Oxalate & $11 / 13 / 2012$ & 2.05 & 2 & 0.05 & 2.5 \\
\hline Soluble Oxalate & $11 / 14 / 2012$ & 2.05 & 2 & 0.05 & 2.5 \\
\hline Soluble Oxalate & $11 / 16 / 2012$ & 2.04 & 2 & 0.04 & 2 \\
\hline Soluble Oxalate & $11 / 16 / 2012$ & 2.08 & 2 & 0.08 & 4 \\
\hline Soluble Oxalate & $11 / 17 / 2012$ & 1.99 & 2 & \begin{tabular}{|c|}
-0.01 \\
\end{tabular} & -0.5 \\
\hline Soluble Oxalate & $11 / 18 / 2012$ & 2.03 & 2 & 0.03 & 1.5 \\
\hline Soluble Oxalate & $11 / 19 / 2012$ & 2.04 & 2 & 0.04 & 2 \\
\hline Soluble Oxalate & $11 / 20 / 2012$ & 2.03 & 2 & 0.03 & 1.5 \\
\hline Soluble Oxalate & $11 / 21 / 2012$ & 1.9 & 2 & -0.1 & -5 \\
\hline Soluble Oxalate & $11 / 21 / 2012$ & 2.09 & 2 & 0.09 & 4.5 \\
\hline Soluble Oxalate & $11 / 21 / 2012$ & 2.09 & 2 & 0.09 & 4.5 \\
\hline Soluble Oxalate & $11 / 22 / 2012$ & 1.99 & 2 & -0.01 & -0.5 \\
\hline Soluble Oxalate & $11 / 24 / 2012$ & 1.92 & 2 & -0.08 & -4 \\
\hline Soluble Oxalate & $11 / 24 / 2012$ & 1.92 & 2 & -0.08 & -4 \\
\hline
\end{tabular}


SRNL-STI-2013-00139

Revision 0

Table A1. DWPF Laboratory Measurements of Anion and TOC Standards

\begin{tabular}{|c|c|c|c|c|c|}
\hline Analyte & Date & \begin{tabular}{|c|}
$\begin{array}{c}\text { Measurement } \\
(\mathrm{ppm})\end{array}$ \\
\end{tabular} & $\begin{array}{c}\text { Reference } \\
\text { Value (ppm) }\end{array}$ & diff & $\begin{array}{c}\text { \% rel } \\
\text { diff }\end{array}$ \\
\hline Soluble Oxalate & $11 / 24 / 2012$ & $\begin{array}{r}1.99 \\
\end{array}$ & 2 & -0.01 & -0.5 \\
\hline Soluble Oxalate & $11 / 24 / 2012$ & 2.03 & 2 & \begin{tabular}{|l|}
0.03 \\
\end{tabular} & 1.5 \\
\hline Soluble Oxalate & $11 / 25 / 2012$ & 1.99 & 2 & $\mid-0.01$ & -0.5 \\
\hline Soluble Oxalate & $11 / 25 / 2012$ & 1.99 & 2 & \begin{tabular}{|l|}
-0.01 \\
\end{tabular} & -0.5 \\
\hline Soluble Oxalate & $11 / 26 / 2012$ & 2.19 & 2 & \begin{tabular}{|l|}
0.19 \\
\end{tabular} & 9.5 \\
\hline Soluble Oxalate & $11 / 26 / 2012$ & 2.2 & 2 & $\begin{array}{l}0.2 \\
\end{array}$ & 10 \\
\hline Soluble Oxalate & $11 / 27 / 2012$ & 2.22 & 2 & 0.22 & 11 \\
\hline Soluble Oxalate & $11 / 28 / 2012$ & 2.13 & 2 & 0.13 & 6.5 \\
\hline Soluble Oxalate & $11 / 28 / 2012$ & 2.22 & 2 & 0.22 & 11 \\
\hline Soluble Oxalate & $11 / 29 / 2012$ & 2.21 & 2 & 0.21 & 10.5 \\
\hline Soluble Oxalate & $11 / 30 / 2012$ & 2.11 & 2 & 0.11 & 5.5 \\
\hline Soluble Oxalate & $12 / 02 / 2012$ & 2.08 & 2 & 0.08 & 4 \\
\hline Soluble Oxalate & $12 / 02 / 2012$ & 2.12 & 2 & 0.12 & 6 \\
\hline Soluble Oxalate & $12 / 03 / 2012$ & 2.09 & 2 & 0.09 & 4.5 \\
\hline Soluble Oxalate & $12 / 03 / 2012$ & 2.11 & 2 & 0.11 & 5.5 \\
\hline Soluble Oxalate & $12 / 04 / 2012$ & 1.93 & 2 & -0.07 & -3.5 \\
\hline Soluble Oxalate & $12 / 04 / 2012$ & 2.03 & 2 & \begin{tabular}{|l|}
0.03 \\
\end{tabular} & 1.5 \\
\hline Soluble Oxalate & $12 / 06 / 2012$ & 2.04 & 2 & 0.04 & 2 \\
\hline Soluble Oxalate & $12 / 06 / 2012$ & 2.09 & 2 & 0.09 & 4.5 \\
\hline Soluble Oxalate & $12 / 07 / 2012$ & 1.94 & 2 & -0.06 & -3 \\
\hline Soluble Oxalate & $12 / 08 / 2012$ & 2.05 & 2 & \begin{tabular}{|l|}
0.05 \\
\end{tabular} & 2.5 \\
\hline Soluble Oxalate & $12 / 09 / 2012$ & 2.05 & 2 & 0.05 & 2.5 \\
\hline Soluble Oxalate & $12 / 10 / 2012$ & 2.07 & 2 & 0.07 & 3.5 \\
\hline Soluble Oxalate & $12 / 11 / 2012$ & 1.98 & 2 & -0.02 & -1 \\
\hline Soluble Oxalate & $12 / 12 / 2012$ & 1.9 & 2 & \begin{tabular}{|l|}
-0.1 \\
\end{tabular} & -5 \\
\hline Soluble Oxalate & $12 / 12 / 2012$ & 1.98 & 2 & -0.02 & -1 \\
\hline Soluble Oxalate & $12 / 14 / 2012$ & 2.07 & 2 & \begin{tabular}{|l|}
0.07 \\
\end{tabular} & 3.5 \\
\hline Soluble Oxalate & $12 / 14 / 2012$ & 2.07 & 2 & 0.07 & 3.5 \\
\hline Soluble Oxalate & $12 / 15 / 2012$ & 1.99 & 2 & -0.01 & -0.5 \\
\hline Soluble Oxalate & $12 / 15 / 2012$ & 2.06 & 2 & 0.06 & 3 \\
\hline Soluble Oxalate & $12 / 15 / 2012$ & 2.06 & 2 & 0.06 & 3 \\
\hline Soluble Oxalate & $12 / 16 / 2012$ & 2.06 & 2 & 0.06 & 3 \\
\hline Soluble Oxalate & $12 / 17 / 2012$ & 2.06 & 2 & 0.06 & 3 \\
\hline Soluble Oxalate & $12 / 18 / 2012$ & 2.04 & 2 & 0.04 & 2 \\
\hline Soluble Oxalate & $12 / 20 / 2012$ & 2.08 & 2 & 0.08 & 4 \\
\hline Soluble Oxalate & $12 / 20 / 2012$ & 2.08 & 2 & 0.08 & 4 \\
\hline Soluble Oxalate & $12 / 21 / 2012$ & 2.06 & 2 & 0.06 & 3 \\
\hline Soluble Oxalate & $12 / 21 / 2012$ & 2.07 & 2 & 0.07 & 3.5 \\
\hline Soluble Oxalate & $12 / 22 / 2012$ & 2.1 & 2 & 0.1 & 5 \\
\hline Soluble Oxalate & $12 / 23 / 2012$ & 2.04 & 2 & 0.04 & 2 \\
\hline Soluble Oxalate & $12 / 23 / 2012$ & 2.06 & 2 & 0.06 & 3 \\
\hline Soluble Oxalate & $12 / 23 / 2012$ & 2.1 & 2 & 0.1 & 5 \\
\hline Soluble Oxalate & $12 / 23 / 2012$ & 2.1 & 2 & 0.1 & 5 \\
\hline Soluble Oxalate & $12 / 24 / 2012$ & 2 & 2 & 0 & 0 \\
\hline Soluble Oxalate & $12 / 24 / 2012$ & 2.09 & 2 & 0.09 & 4.5 \\
\hline Soluble Oxalate & $12 / 25 / 2012$ & 2.05 & 2 & 0.05 & 2.5 \\
\hline Soluble Oxalate & $12 / 25 / 2012$ & 2.05 & 2 & 0.05 & 2.5 \\
\hline Soluble Oxalate & $12 / 27 / 2012$ & 2.06 & 2 & 0.06 & 3 \\
\hline Soluble Oxalate & $12 / 28 / 2012$ & 2.09 & 2 & 0.09 & 4.5 \\
\hline Soluble Oxalate & $12 / 28 / 2012$ & 2.09 & 2 & 0.09 & 4.5 \\
\hline Soluble Oxalate & $12 / 29 / 2012$ & 2.04 & 2 & 0.04 & 2 \\
\hline Soluble Oxalate & $12 / 29 / 2012$ & 2.04 & 2 & 0.04 & 2 \\
\hline Soluble Oxalate & $12 / 29 / 2012$ & 2.1 & 2 & 0.1 & 5 \\
\hline Soluble Oxalate & $12 / 30 / 2012$ & 2.08 & 2 & 0.08 & 4 \\
\hline Soluble Oxalate & $12 / 31 / 2012$ & 2.03 & 2 & 0.03 & 1.5 \\
\hline Soluble Oxalate & $12 / 31 / 2012$ & 2.07 & 2 & 0.07 & 3.5 \\
\hline Soluble Oxalate & $01 / 01 / 2013$ & 2.07 & 2 & 0.07 & 3.5 \\
\hline Soluble Oxalate & $01 / 01 / 2013$ & 2.08 & 2 & 0.08 & 4 \\
\hline Soluble Oxalate & $01 / 02 / 2013$ & 2.07 & 2 & 0.07 & 3.5 \\
\hline Soluble Oxalate & $01 / 03 / 2013$ & 2.05 & 2 & 0.05 & 2.5 \\
\hline Soluble Oxalate & $01 / 03 / 2013$ & 2.05 & 2 & 0.05 & 2.5 \\
\hline Soluble Oxalate & $01 / 04 / 2013$ & 2.02 & 2 & 0.02 & 1 \\
\hline Soluble Oxalate & $01 / 05 / 2013$ & 2.04 & 2 & 0.04 & 2 \\
\hline Soluble Oxalate & $01 / 05 / 2013$ & 2.06 & 2 & 0.06 & 3 \\
\hline Soluble Oxalate & $01 / 06 / 2013$ & 2 & 2 & 0 & 0 \\
\hline Soluble Oxalate & 01/07/2013 & 1.83 & 2 & -0.17 & -8.5 \\
\hline Soluble Oxalate & $01 / 08 / 2013$ & 1.97 & 2 & \begin{tabular}{|l|}
-0.03 \\
\end{tabular} & -1.5 \\
\hline Soluble Oxalate & $01 / 11 / 2013$ & 1.85 & 2 & -0.15 & -7.5 \\
\hline Soluble Oxalate & $01 / 11 / 2013$ & 2.04 & 2 & \begin{tabular}{|l|}
0.04 \\
\end{tabular} & 2 \\
\hline Soluble Oxalate & $01 / 12 / 2013$ & 1.83 & 2 & -0.17 & -8.5 \\
\hline Soluble Oxalate & $01 / 12 / 2013$ & 1.93 & 2 & $\mid-0.07$ & -3.5 \\
\hline Soluble Oxalate & $01 / 13 / 2013$ & 2.01 & 2 & 0.01 & 0.5 \\
\hline Soluble Oxalate & $01 / 13 / 2013$ & 2.02 & 2 & 0.02 & 1 \\
\hline Soluble Oxalate & $01 / 13 / 2013$ & 2.04 & 2 & 0.04 & 2 \\
\hline Soluble Oxalate & $01 / 14 / 2013$ & 2.02 & 2 & 0.02 & 1 \\
\hline Soluble Oxalate & $01 / 14 / 2013$ & 2.06 & 2 & 0.06 & 3 \\
\hline Soluble Oxalate & $01 / 14 / 2013$ & 2.07 & 2 & 0.07 & 3.5 \\
\hline Soluble Oxalate & $01 / 15 / 2013$ & 2.15 & 2 & 0.15 & 7.5 \\
\hline Soluble Oxalate & $01 / 17 / 2013$ & 2.15 & 2 & 0.15 & 7.5 \\
\hline Soluble Oxalate & $01 / 18 / 2013$ & 2.11 & 2 & 0.11 & 5.5 \\
\hline Soluble Oxalate & $01 / 18 / 2013$ & 2.18 & 2 & 0.18 & 9 \\
\hline Soluble Oxalate & $01 / 18 / 2013$ & 2.11 & 2 & 0.11 & 5.5 \\
\hline Soluble Oxalate & $01 / 19 / 2013$ & 2.12 & 2 & \begin{tabular}{|l|}
0.12 \\
\end{tabular} & 6 \\
\hline
\end{tabular}

\begin{tabular}{|c|c|c|c|c|c|}
\hline Analyte & Date & $\begin{array}{l}\text { Measurement } \\
\text { (ppm) }\end{array}$ & $\begin{array}{c}\text { Reference } \\
\text { Value (ppm) }\end{array}$ & diff & $\begin{array}{c}\text { \% rel } \\
\text { diff }\end{array}$ \\
\hline Soluble Oxalate & $01 / 19 / 2013$ & 2.19 & 2 & 0.19 & 9.5 \\
\hline Soluble Oxalate & $01 / 20 / 2013$ & 2.16 & 2 & 0.16 & 8 \\
\hline Soluble Oxalate & $01 / 21 / 2013$ & 2.12 & 2 & 0.12 & 6 \\
\hline Soluble Oxalate & $01 / 22 / 2013$ & 2.1 & 2 & 0.1 & 5 \\
\hline \begin{tabular}{|c|} 
Soluble Oxalate \\
\end{tabular} & $01 / 22 / 2013$ & 2.13 & 2 & 0.13 & 6.5 \\
\hline Soluble Oxalate & $01 / 23 / 2013$ & 2.07 & 2 & 0.07 & 3.5 \\
\hline Soluble Oxalate & $01 / 23 / 2013$ & 2.07 & 2 & 0.07 & 3.5 \\
\hline Soluble Oxalate & $01 / 23 / 2013$ & 2.1 & 2 & 0.1 & 5 \\
\hline Soluble Oxalate & $01 / 23 / 2013$ & 2.1 & 2 & 0.1 & 5 \\
\hline Soluble Oxalate & $01 / 24 / 2013$ & 2.04 & 2 & 0.04 & 2 \\
\hline Soluble Oxalate & $01 / 24 / 2013$ & 2.09 & 2 & 0.09 & 4.5 \\
\hline Soluble Oxalate & $01 / 25 / 2013$ & 2.02 & 2 & 0.02 & 1 \\
\hline Soluble Oxalate & $01 / 25 / 2013$ & 2.13 & 2 & 0.13 & 6.5 \\
\hline Soluble Oxalate & $01 / 25 / 2013$ & 2.17 & 2 & 0.17 & 8.5 \\
\hline Soluble Oxalate & $01 / 26 / 2013$ & 2.17 & 2 & 0.17 & 8.5 \\
\hline Soluble Oxalate & $01 / 26 / 2013$ & 2.17 & 2 & 0.17 & 8.5 \\
\hline Soluble Oxalate & $01 / 26 / 2013$ & 2.17 & 2 & 0.17 & 8.5 \\
\hline Soluble Oxalate & $01 / 26 / 2013$ & 2.18 & 2 & 0.18 & 9 \\
\hline Soluble Oxalate & $01 / 26 / 2013$ & 2.18 & 2 & 0.18 & 9 \\
\hline Soluble Oxalate & $01 / 27 / 2013$ & 2.17 & 2 & 0.17 & 8.5 \\
\hline Soluble Oxalate & $01 / 27 / 2013$ & 2.18 & 2 & 0.18 & 9 \\
\hline Soluble Oxalate & $01 / 28 / 2013$ & 2.05 & 2 & 0.05 & 2.5 \\
\hline Soluble Oxalate & $01 / 28 / 2013$ & 2.17 & 2 & 0.17 & 8.5 \\
\hline Soluble Oxalate & $01 / 28 / 2013$ & 2.17 & 2 & 0.17 & 8.5 \\
\hline Soluble Oxalate & $01 / 29 / 2013$ & 2.15 & 2 & 0.15 & 7.5 \\
\hline Soluble Oxalate & $01 / 30 / 2013$ & 2.13 & 2 & 0.13 & 6.5 \\
\hline Soluble Oxalate & $01 / 31 / 2013$ & 2.13 & 2 & 0.13 & 6.5 \\
\hline Soluble Oxalate & $01 / 31 / 2013$ & 2.14 & 2 & 0.14 & 7 \\
\hline Soluble Oxalate & $01 / 31 / 2013$ & 2.15 & 2 & 0.15 & 7.5 \\
\hline Soluble Oxalate & $02 / 01 / 2013$ & 2.05 & 2 & 0.05 & 2.5 \\
\hline Soluble Oxalate & $02 / 01 / 2013$ & 2.01 & 2 & 0.01 & 0.5 \\
\hline Soluble Oxalate & $02 / 01 / 2013$ & 2.04 & 2 & 0.04 & 2 \\
\hline Soluble Oxalate & $02 / 01 / 2013$ & 2.05 & 2 & 0.05 & 2.5 \\
\hline Soluble Oxalate & $02 / 03 / 2013$ & 2.09 & 2 & 0.09 & 4.5 \\
\hline Soluble Oxalate & $02 / 04 / 2013$ & 2.04 & 2 & 0.04 & 2 \\
\hline Soluble Oxalate & $02 / 04 / 2013$ & 2.09 & 2 & 0.09 & 4.5 \\
\hline Soluble Oxalate & $02 / 05 / 2013$ & 2.06 & 2 & 0.06 & 3 \\
\hline Soluble Oxalate & $02 / 05 / 2013$ & 2.06 & 2 & 0.06 & 3 \\
\hline Soluble Oxalate & 02/06/2013 & 2.12 & 2 & 0.12 & 6 \\
\hline Soluble Oxalate & $02 / 07 / 2013$ & 2.12 & 2 & 0.12 & 6 \\
\hline Soluble Oxalate & $02 / 07 / 2013$ & 2.15 & 2 & 0.15 & 7.5 \\
\hline Soluble Oxalate & $02 / 08 / 2013$ & 2.13 & 2 & 0.13 & 6.5 \\
\hline Soluble Oxalate & $02 / 09 / 2013$ & 2.11 & 2 & 0.11 & 5.5 \\
\hline Soluble Oxalate & $02 / 09 / 2013$ & 2.13 & 2 & 0.13 & 6.5 \\
\hline Soluble Oxalate & $02 / 09 / 2013$ & 2.15 & 2 & 0.15 & 7.5 \\
\hline Soluble Oxalate & $02 / 10 / 2013$ & 2.16 & 2 & 0.16 & 8 \\
\hline Soluble Oxalate & $11 / 09 / 2012$ & 16.27 & 16 & \begin{tabular}{|l|}
0.27 \\
\end{tabular} & 1.6875 \\
\hline Soluble Oxalate & $11 / 10 / 2012$ & 15.99 & 16 & $\mid-0.01$ & -0.0625 \\
\hline Soluble Oxalate & $11 / 11 / 2012$ & 15.89 & 16 & $\mid-0.11$ & -0.6875 \\
\hline Soluble Oxalate & $11 / 13 / 2012$ & 15.69 & 16 & \begin{tabular}{|c|}
-0.31 \\
\end{tabular} & -1.9375 \\
\hline Soluble Oxalate & $11 / 13 / 2012$ & \begin{tabular}{|l|}
15.7 \\
\end{tabular} & 16 & \begin{tabular}{|l|}
-0.3 \\
\end{tabular} & -1.875 \\
\hline Soluble Oxalate & $11 / 14 / 2012$ & 15.63 & 16 & \begin{tabular}{|c|}
-0.37 \\
\end{tabular} & -2.3125 \\
\hline Soluble Oxalate & $11 / 16 / 2012$ & 15.82 & 16 & \begin{tabular}{|c|}
-0.18 \\
\end{tabular} & -1.125 \\
\hline Soluble Oxalate & $11 / 16 / 2012$ & 16.08 & 16 & \begin{tabular}{|l|}
0.08 \\
\end{tabular} & 0.5 \\
\hline Soluble Oxalate & $11 / 17 / 2012$ & 15.98 & 16 & \begin{tabular}{|l|}
-0.02 \\
\end{tabular} & -0.125 \\
\hline Soluble Oxalate & $11 / 18 / 2012$ & 15.81 & 16 & \begin{tabular}{|c|}
-0.19 \\
\end{tabular} & -1.1875 \\
\hline Soluble Oxalate & $11 / 19 / 2012$ & 15.89 & 16 & \begin{tabular}{|c|}
-0.11 \\
\end{tabular} & -0.6875 \\
\hline Soluble Oxalate & $11 / 20 / 2012$ & 15.82 & 16 & -0.18 & -1.125 \\
\hline Soluble Oxalate & $11 / 21 / 2012$ & 14.78 & 16 & -1.22 & -7.625 \\
\hline Soluble Oxalate & $11 / 21 / 2012$ & 15.95 & 16 & \begin{tabular}{|c|}
-0.05 \\
\end{tabular} & -0.3125 \\
\hline Soluble Oxalate & $11 / 21 / 2012$ & 16.11 & 16 & \begin{tabular}{|l|}
0.11 \\
\end{tabular} & 0.6875 \\
\hline Soluble Oxalate & $11 / 22 / 2012$ & 15.3 & 16 & $\mid-0.7$ & -4.375 \\
\hline Soluble Oxalate & $11 / 24 / 2012$ & 15.39 & 16 & \begin{tabular}{|c|}
-0.61 \\
\end{tabular} & -3.8125 \\
\hline Soluble Oxalate & $11 / 24 / 2012$ & 15.87 & 16 & \begin{tabular}{|c|}
-0.13 \\
\end{tabular} & -0.8125 \\
\hline Soluble Oxalate & $11 / 24 / 2012$ & 15.99 & 16 & $\mid-0.01$ & -0.0625 \\
\hline Soluble Oxalate & $11 / 24 / 2012$ & 16.13 & 16 & 0.13 & 0.8125 \\
\hline Soluble Oxalate & $11 / 25 / 2012$ & 15.64 & 16 & -0.36 & -2.25 \\
\hline Soluble Oxalate & $11 / 26 / 2012$ & 15.44 & 16 & -0.56 & -3.5 \\
\hline Soluble Oxalate & $11 / 26 / 2012$ & 15.85 & 16 & \begin{tabular}{|c|}
-0.15 \\
\end{tabular} & -0.9375 \\
\hline Soluble Oxalate & $11 / 27 / 2012$ & 16.16 & 16 & 0.16 & 1 \\
\hline Soluble Oxalate & $11 / 28 / 2012$ & 16.05 & 16 & 0.05 & 0.3125 \\
\hline Soluble Oxalate & $11 / 28 / 2012$ & 16.17 & 16 & 0.17 & 1.0625 \\
\hline Soluble Oxalate & $11 / 28 / 2012$ & 16.17 & 16 & 0.17 & 1.0625 \\
\hline Soluble Oxalate & $11 / 29 / 2012$ & 16.2 & 16 & 0.2 & 1.25 \\
\hline Soluble Oxalate & $11 / 30 / 2012$ & 15.49 & 16 & \begin{tabular}{|l|}
-0.51 \\
\end{tabular} & -3.1875 \\
\hline Soluble Oxalate & $12 / 02 / 2012$ & 15.8 & 16 & -0.2 & -1.25 \\
\hline Soluble Oxalate & $12 / 02 / 2012$ & 15.92 & 16 & \begin{tabular}{|l|}
-0.08 \\
\end{tabular} & -0.5 \\
\hline Soluble Oxalate & $12 / 03 / 2012$ & 15.53 & 16 & -0.47 & -2.9375 \\
\hline Soluble Oxalate & $12 / 03 / 2012$ & 16.09 & 16 & \begin{tabular}{|l|}
0.09 \\
\end{tabular} & 0.5625 \\
\hline Soluble Oxalate & $12 / 04 / 2012$ & 15.72 & 16 & -0.28 & -1.75 \\
\hline Soluble Oxalate & $12 / 04 / 2012$ & 16.13 & 16 & \begin{tabular}{|l|}
0.13 \\
\end{tabular} & 0.8125 \\
\hline Soluble Oxalate & $12 / 06 / 2012$ & 16.05 & 16 & 0.05 & 0.3125 \\
\hline Soluble Oxalate & $12 / 06 / 2012$ & 16.1 & 16 & 0.1 & 0.625 \\
\hline
\end{tabular}


SRNL-STI-2013-00139

Revision 0

Table A1. DWPF Laboratory Measurements of Anion and TOC Standards

\begin{tabular}{|c|c|c|c|c|c|}
\hline Analyte & Date & \begin{tabular}{|c|}
$\begin{array}{c}\text { Measurement } \\
(\mathrm{ppm})\end{array}$ \\
\end{tabular} & $\begin{array}{c}\text { Reference } \\
\text { Value (ppm) }\end{array}$ & diff & $\begin{array}{l}\text { \% rel } \\
\text { diff }\end{array}$ \\
\hline Soluble Oxalate & $12 / 07 / 2012$ & 14.62 & 16 & -1.38 & -8.625 \\
\hline Soluble Oxalate & $12 / 08 / 2012$ & 15.59 & 16 & -0.41 & -2.5625 \\
\hline Soluble Oxalate & $12 / 09 / 2012$ & 15.98 & 16 & -0.02 & -0.125 \\
\hline Soluble Oxalate & $12 / 10 / 2012$ & 16.11 & 16 & 0.11 & 0.6875 \\
\hline Soluble Oxalate & $12 / 11 / 2012$ & 15.6 & 16 & -0.4 & -2.5 \\
\hline Soluble Oxalate & $12 / 12 / 2012$ & 14.89 & 16 & -1.11 & -6.9375 \\
\hline Soluble Oxalate & $12 / 12 / 2012$ & 15.34 & 16 & -0.66 & -4.125 \\
\hline Soluble Oxalate & $12 / 14 / 2012$ & 15.98 & 16 & -0.02 & -0.125 \\
\hline Soluble Oxalate & $12 / 14 / 2012$ & 16.03 & 16 & 0.03 & 0.1875 \\
\hline Soluble Oxalate & $12 / 15 / 2012$ & 15.7 & 16 & -0.3 & -1.875 \\
\hline Soluble Oxalate & $12 / 15 / 2012$ & 15.83 & 16 & -0.17 & -1.0625 \\
\hline Soluble Oxalate & $12 / 15 / 2012$ & 15.83 & 16 & -0.17 & -1.0625 \\
\hline Soluble Oxalate & $12 / 16 / 2012$ & 15.85 & 16 & -0.15 & -0.9375 \\
\hline Soluble Oxalate & $12 / 17 / 2012$ & 15.82 & 16 & -0.18 & -1.125 \\
\hline Soluble Oxalate & $12 / 18 / 2012$ & 15.86 & 16 & -0.14 & -0.875 \\
\hline Soluble Oxalate & $12 / 20 / 2012$ & 15.67 & 16 & -0.33 & -2.0625 \\
\hline Soluble Oxalate & $12 / 20 / 2012$ & 15.68 & 16 & -0.32 & -2 \\
\hline Soluble Oxalate & $12 / 21 / 2012$ & 15.99 & 16 & -0.01 & -0.0625 \\
\hline Soluble Oxalate & $12 / 21 / 2012$ & 15.99 & 16 & -0.01 & -0.0625 \\
\hline Soluble Oxalate & $12 / 22 / 2012$ & 16.05 & 16 & 0.05 & 0.3125 \\
\hline Soluble Oxalate & $12 / 23 / 2012$ & 15.49 & 16 & -0.51 & -3.1875 \\
\hline Soluble Oxalate & $12 / 23 / 2012$ & 15.99 & 16 & -0.01 & -0.0625 \\
\hline Soluble Oxalate & $12 / 23 / 2012$ & 16.03 & 16 & 0.03 & 0.1875 \\
\hline Soluble Oxalate & $12 / 23 / 2012$ & 16.03 & 16 & 0.03 & 0.1875 \\
\hline Soluble Oxalate & $12 / 24 / 2012$ & 16 & 16 & 0 & 0 \\
\hline Soluble Oxalate & $12 / 24 / 2012$ & 16.04 & 16 & 0.04 & 0.25 \\
\hline Soluble Oxalate & $12 / 25 / 2012$ & 15.76 & 16 & -0.24 & -1.5 \\
\hline Soluble Oxalate & $12 / 27 / 2012$ & 16.08 & 16 & 0.08 & 0.5 \\
\hline Soluble Oxalate & $12 / 28 / 2012$ & 15.6 & 16 & -0.4 & -2.5 \\
\hline Soluble Oxalate & $12 / 28 / 2012$ & 16.02 & 16 & 0.02 & 0.125 \\
\hline Soluble Oxalate & $12 / 28 / 2012$ & 16.02 & 16 & 0.02 & 0.125 \\
\hline Soluble Oxalate & $12 / 29 / 2012$ & 15.93 & 16 & -0.07 & -0.4375 \\
\hline Soluble Oxalate & $12 / 29 / 2012$ & 15.93 & 16 & -0.07 & -0.4375 \\
\hline Soluble Oxalate & $12 / 29 / 2012$ & 16.04 & 16 & 0.04 & 0.25 \\
\hline Soluble Oxalate & $12 / 30 / 2012$ & 16.09 & 16 & 0.09 & 0.5625 \\
\hline Soluble Oxalate & $12 / 31 / 2012$ & 16.02 & 16 & 0.02 & 0.125 \\
\hline Soluble Oxalate & $12 / 31 / 2012$ & 16.08 & 16 & 0.08 & 0.5 \\
\hline Soluble Oxalate & $01 / 01 / 2013$ & 15.86 & 16 & -0.14 & -0.875 \\
\hline Soluble Oxalate & $01 / 01 / 2013$ & 16.08 & 16 & 0.08 & 0.5 \\
\hline Soluble Oxalate & $01 / 02 / 2013$ & 15.85 & 16 & -0.15 & -0.9375 \\
\hline Soluble Oxalate & $01 / 03 / 2013$ & 15.84 & 16 & -0.16 & -1 \\
\hline Soluble Oxalate & $01 / 03 / 2013$ & 15.84 & 16 & -0.16 & -1 \\
\hline Soluble Oxalate & $01 / 04 / 2013$ & 15.57 & 16 & -0.43 & -2.6875 \\
\hline Soluble Oxalate & $01 / 05 / 2013$ & 15.53 & 16 & -0.47 & -2.9375 \\
\hline Soluble Oxalate & $01 / 05 / 2013$ & 15.85 & 16 & -0.15 & -0.9375 \\
\hline Soluble Oxalate & $01 / 06 / 2013$ & 15.39 & 16 & -0.61 & -3.8125 \\
\hline Soluble Oxalate & 01/07/2013 & 15.34 & 16 & -0.66 & -4.125 \\
\hline Soluble Oxalate & $01 / 08 / 2013$ & 15.46 & 16 & -0.54 & -3.375 \\
\hline Soluble Oxalate & $01 / 11 / 2013$ & 14.7 & 16 & -1.3 & -8.125 \\
\hline Soluble Oxalate & $01 / 11 / 2013$ & 15.81 & 16 & -0.19 & -1.1875 \\
\hline Soluble Oxalate & $01 / 12 / 2013$ & 14.44 & 16 & -1.56 & -9.75 \\
\hline Soluble Oxalate & $01 / 12 / 2013$ & 14.78 & 16 & -1.22 & -7.625 \\
\hline Soluble Oxalate & $01 / 13 / 2013$ & 14.43 & 16 & -1.57 & -9.8125 \\
\hline Soluble Oxalate & $01 / 13 / 2013$ & 14.67 & 16 & -1.33 & -8.3125 \\
\hline Soluble Oxalate & $01 / 13 / 2013$ & 15.65 & 16 & -0.35 & -2.1875 \\
\hline Soluble Oxalate & $01 / 14 / 2013$ & 14.65 & 16 & -1.35 & -8.4375 \\
\hline Soluble Oxalate & $01 / 14 / 2013$ & 14.65 & 16 & -1.35 & -8.4375 \\
\hline Soluble Oxalate & $01 / 14 / 2013$ & 15.17 & 16 & -0.83 & -5.1875 \\
\hline Soluble Oxalate & $01 / 15 / 2013$ & 15.15 & 16 & -0.85 & -5.3125 \\
\hline Soluble Oxalate & $01 / 17 / 2013$ & 16.2 & 16 & 0.2 & 1.25 \\
\hline Soluble Oxalate & $01 / 18 / 2013$ & 15.55 & 16 & -0.45 & -2.8125 \\
\hline Soluble Oxalate & $01 / 18 / 2013$ & 16.23 & 16 & 0.23 & 1.4375 \\
\hline Soluble Oxalate & $01 / 18 / 2013$ & 16.48 & 16 & 0.48 & 3 \\
\hline Soluble Oxalate & $01 / 19 / 2013$ & 16.45 & 16 & 0.45 & 2.8125 \\
\hline Soluble Oxalate & $01 / 19 / 2013$ & 16.5 & 16 & 0.5 & 3.125 \\
\hline Soluble Oxalate & $01 / 20 / 2013$ & 16.22 & 16 & 0.22 & 1.375 \\
\hline Soluble Oxalate & $01 / 21 / 2013$ & 16.11 & 16 & 0.11 & 0.6875 \\
\hline Soluble Oxalate & $01 / 22 / 2013$ & 16.25 & 16 & 0.25 & 1.5625 \\
\hline Soluble Oxalate & $01 / 22 / 2013$ & 16.31 & 16 & 0.31 & 1.9375 \\
\hline Soluble Oxalate & $01 / 23 / 2013$ & 16.16 & 16 & 0.16 & 1 \\
\hline Soluble Oxalate & $01 / 23 / 2013$ & 16.22 & 16 & 0.22 & 1.375 \\
\hline Soluble Oxalate & $01 / 23 / 2013$ & 16.33 & 16 & 0.33 & 2.0625 \\
\hline Soluble Oxalate & $01 / 23 / 2013$ & 16.33 & 16 & 0.33 & 2.0625 \\
\hline Soluble Oxalate & $01 / 24 / 2013$ & 16.54 & 16 & 0.54 & 3.375 \\
\hline Soluble Oxalate & $01 / 24 / 2013$ & 16.62 & 16 & 0.62 & 3.875 \\
\hline Soluble Oxalate & $01 / 25 / 2013$ & 16.45 & 16 & 0.45 & 2.8125 \\
\hline Soluble Oxalate & $01 / 25 / 2013$ & 16.47 & 16 & 0.47 & 2.9375 \\
\hline Soluble Oxalate & $01 / 25 / 2013$ & 16.55 & 16 & 0.55 & 3.4375 \\
\hline Soluble Oxalate & $01 / 26 / 2013$ & 16.28 & 16 & 0.28 & 1.75 \\
\hline Soluble Oxalate & $01 / 26 / 2013$ & 16.45 & 16 & 0.45 & 2.8125 \\
\hline Soluble Oxalate & $01 / 26 / 2013$ & 16.46 & 16 & 0.46 & 2.875 \\
\hline Soluble Oxalate & $01 / 26 / 2013$ & 16.48 & 16 & 0.48 & 3 \\
\hline Soluble Oxalate & $01 / 26 / 2013$ & 16.49 & 16 & 0.49 & 3.0625 \\
\hline
\end{tabular}

\begin{tabular}{|c|c|c|c|c|c|}
\hline Analyte & Date & \begin{tabular}{|c|}
$\begin{array}{c}\text { Measurement } \\
\text { (ppm) }\end{array}$ \\
\end{tabular} & $\begin{array}{c}\text { Reference } \\
\text { Value (ppm) }\end{array}$ & diff & $\begin{array}{c}\text { \% rel } \\
\text { diff }\end{array}$ \\
\hline Soluble Oxalate & $01 / 27 / 2013$ & \begin{tabular}{r|}
16.48 \\
\end{tabular} & 16 & 0.48 & 3 \\
\hline Soluble Oxalate & $01 / 27 / 2013$ & 16.48 & 16 & 0.48 & 3 \\
\hline Soluble Oxalate & $01 / 28 / 2013$ & 15.73 & 16 & -0.27 & -1.6875 \\
\hline Soluble Oxalate & $01 / 28 / 2013$ & 16.41 & 16 & 0.41 & 2.5625 \\
\hline Soluble Oxalate & $01 / 28 / 2013$ & 16.41 & 16 & 0.41 & 2.5625 \\
\hline Soluble Oxalate & $01 / 29 / 2013$ & 16.37 & 16 & 0.37 & 2.3125 \\
\hline Soluble Oxalate & $01 / 30 / 2013$ & 16.34 & 16 & 0.34 & 2.125 \\
\hline Soluble Oxalate & $01 / 31 / 2013$ & 15.81 & 16 & \begin{tabular}{|l|}
-0.19 \\
\end{tabular} & \begin{tabular}{|l|}
-1.1875 \\
\end{tabular} \\
\hline Soluble Oxalate & $01 / 31 / 2013$ & 16.32 & 16 & 0.32 & 2 \\
\hline Soluble Oxalate & $01 / 31 / 2013$ & 16.33 & 16 & 0.33 & 2.0625 \\
\hline Soluble Oxalate & $02 / 01 / 2013$ & 15.72 & 16 & -0.28 & -1.75 \\
\hline Soluble Oxalate & $02 / 01 / 2013$ & 15.72 & 16 & -0.28 & -1.75 \\
\hline Soluble Oxalate & $02 / 01 / 2013$ & 16.33 & 16 & 0.33 & 2.0625 \\
\hline Soluble Oxalate & $02 / 01 / 2013$ & 16.37 & 16 & 0.37 & 2.3125 \\
\hline Soluble Oxalate & $02 / 03 / 2013$ & 16.41 & 16 & 0.41 & 2.5625 \\
\hline Soluble Oxalate & $02 / 04 / 2013$ & 15.76 & 16 & \begin{tabular}{|l|}
-0.24 \\
\end{tabular} & -1.5 \\
\hline Soluble Oxalate & $02 / 04 / 2013$ & 16.38 & 16 & 0.38 & 2.375 \\
\hline Soluble Oxalate & $02 / 05 / 2013$ & 15.26 & 16 & -0.74 & -4.625 \\
\hline Soluble Oxalate & $02 / 05 / 2013$ & 16.43 & 16 & 0.43 & 2.6875 \\
\hline Soluble Oxalate & $02 / 06 / 2013$ & 16.09 & 16 & 0.09 & 0.5625 \\
\hline Soluble Oxalate & $02 / 07 / 2013$ & 16.07 & 16 & 0.07 & 0.4375 \\
\hline Soluble Oxalate & $02 / 07 / 2013$ & 16.31 & 16 & 0.31 & 1.9375 \\
\hline Soluble Oxalate & $02 / 08 / 2013$ & 16.34 & 16 & 0.34 & 2.125 \\
\hline Soluble Oxalate & $02 / 09 / 2013$ & 16.22 & 16 & 0.22 & 1.375 \\
\hline Soluble Oxalate & $02 / 09 / 2013$ & 16.29 & 16 & 0.29 & 1.8125 \\
\hline Soluble Oxalate & $02 / 09 / 2013$ & 16.32 & 16 & 0.32 & 2 \\
\hline Soluble Oxalate & $02 / 10 / 2013$ & 16.38 & 16 & 0.38 & 2.375 \\
\hline TOC 1 & $10 / 15 / 2012$ & 0.97 & 1 & -0.03 & -3 \\
\hline TOC 2 & \begin{tabular}{|l|l|}
$10 / 15 / 2012$ \\
\end{tabular} & 0.98 & 1 & \begin{tabular}{|l|}
-0.02 \\
\end{tabular} & -2 \\
\hline TOC 1 & $10 / 15 / 2012$ & 1 & 1 & 0 & 0 \\
\hline TOC 2 & $10 / 15 / 2012$ & 1.01 & 1 & 0.01 & 1 \\
\hline TOC 1 & $10 / 24 / 2012$ & 0.96 & 1 & -0.04 & -4 \\
\hline TOC 1 & $10 / 24 / 2012$ & 0.97 & 1 & -0.03 & -3 \\
\hline TOC 2 & $10 / 24 / 2012$ & 0.97 & 1 & \begin{tabular}{|l|}
-0.03 \\
\end{tabular} & -3 \\
\hline TOC 2 & $10 / 24 / 2012$ & 0.99 & 1 & \begin{tabular}{|l|}
-0.01 \\
\end{tabular} & -1 \\
\hline TOC 1 & $11 / 21 / 2012$ & 0.96 & 1 & -0.04 & -4 \\
\hline TOC 2 & $11 / 21 / 2012$ & 0.96 & 1 & \begin{tabular}{|l|}
-0.04 \\
\end{tabular} & -4 \\
\hline TOC 1 & $11 / 21 / 2012$ & 0.96 & 1 & \begin{tabular}{|l|}
-0.04 \\
\end{tabular} & -4 \\
\hline TOC 2 & $11 / 21 / 2012$ & 0.97 & 1 & -0.03 & -3 \\
\hline TOC 1 & $11 / 25 / 2012$ & 0.99 & 1 & \begin{tabular}{|l|}
-0.01 \\
\end{tabular} & -1 \\
\hline TOC 1 & $11 / 25 / 2012$ & 0.99 & 1 & \begin{tabular}{|l|}
-0.01 \\
\end{tabular} & -1 \\
\hline TOC 2 & $11 / 25 / 2012$ & 1 & 1 & 0 & 0 \\
\hline TOC 2 & $11 / 25 / 2012$ & 1.01 & 1 & 0.01 & 1 \\
\hline TOC 1 & $12 / 04 / 2012$ & 1.04 & 1 & 0.04 & 4 \\
\hline TOC 2 & $12 / 04 / 2012$ & 1.04 & 1 & 0.04 & 4 \\
\hline TOC 1 & $12 / 04 / 2012$ & 1.05 & 1 & 0.05 & 5 \\
\hline TOC 2 & $12 / 04 / 2012$ & 1.05 & 1 & 0.05 & 5 \\
\hline TOC 1 & $12 / 06 / 2012$ & 1.01 & 1 & 0.01 & 1 \\
\hline TOC 1 & $12 / 06 / 2012$ & 1.02 & 1 & 0.02 & 2 \\
\hline TOC 2 & $12 / 06 / 2012$ & 1.02 & 1 & 0.02 & 2 \\
\hline TOC 2 & $12 / 06 / 2012$ & 1.02 & 1 & 0.02 & 2 \\
\hline TOC 1 & $12 / 09 / 2012$ & 0.98 & 1 & -0.02 & -2 \\
\hline TOC 2 & $12 / 09 / 2012$ & 0.99 & 1 & \begin{tabular}{|l|}
-0.01 \\
\end{tabular} & -1 \\
\hline TOC 2 & $12 / 09 / 2012$ & 0.99 & 1 & \begin{tabular}{|l|}
-0.01 \\
\end{tabular} & -1 \\
\hline TOC 1 & $12 / 09 / 2012$ & 1.01 & 1 & 0.01 & 1 \\
\hline TOC 1 & $12 / 13 / 2012$ & 0.98 & 1 & \begin{tabular}{|l|}
-0.02 \\
\end{tabular} & -2 \\
\hline TOC 1 & $12 / 13 / 2012$ & 1 & 1 & 0 & 0 \\
\hline TOC 2 & $12 / 13 / 2012$ & 1 & 1 & 0 & 0 \\
\hline TOC 2 & $12 / 13 / 2012$ & 1.03 & 1 & 0.03 & 3 \\
\hline TOC 2 & $12 / 14 / 2012$ & 0.9 & 1 & -0.1 & -10 \\
\hline TOC 1 & $12 / 14 / 2012$ & 0.96 & 1 & \begin{tabular}{|l|}
-0.04 \\
\end{tabular} & -4 \\
\hline TOC 1 & $12 / 14 / 2012$ & 1.06 & 1 & 0.06 & 6 \\
\hline TOC 2 & $12 / 14 / 2012$ & 1.06 & 1 & 0.06 & 6 \\
\hline TOC 1 & $12 / 23 / 2012$ & 0.96 & 1 & \begin{tabular}{|l|}
-0.04 \\
\end{tabular} & -4 \\
\hline TOC 2 & $12 / 23 / 2012$ & 0.98 & 1 & -0.02 & -2 \\
\hline TOC 1 & $12 / 23 / 2012$ & 0.99 & 1 & \begin{tabular}{|l|}
-0.01 \\
\end{tabular} & -1 \\
\hline TOC 2 & $12 / 23 / 2012$ & 0.99 & 1 & \begin{tabular}{|l|}
-0.01 \\
\end{tabular} & -1 \\
\hline TOC 1 & $12 / 24 / 2012$ & 0.98 & 1 & -0.02 & -2 \\
\hline TOC 1 & $12 / 24 / 2012$ & 0.98 & 1 & -0.02 & -2 \\
\hline TOC 2 & $12 / 24 / 2012$ & 0.99 & 1 & \begin{tabular}{|l|}
-0.01 \\
\end{tabular} & -1 \\
\hline TOC 2 & $12 / 24 / 2012$ & 0.99 & 1 & \begin{tabular}{|l|}
-0.01 \\
\end{tabular} & -1 \\
\hline TOC 1 & $12 / 25 / 2012$ & 1 & 1 & 0 & 0 \\
\hline TOC 1 & $12 / 25 / 2012$ & 1 & 1 & 0 & 0 \\
\hline TOC 2 & $12 / 25 / 2012$ & 1.04 & 1 & 0.04 & 4 \\
\hline TOC 2 & $12 / 25 / 2012$ & 1.05 & 1 & 0.05 & 5 \\
\hline TOC 1 & $01 / 12 / 2013$ & 0.96 & 1 & \begin{tabular}{|l|}
-0.04 \\
\end{tabular} & -4 \\
\hline TOC 2 & \begin{tabular}{|l|l|}
$01 / 2013$ \\
\end{tabular} & 0.97 & 1 & \begin{tabular}{|l|}
-0.03 \\
\end{tabular} & -3 \\
\hline TOC 2 & $01 / 12 / 2013$ & 0.97 & 1 & \begin{tabular}{|l|}
-0.03 \\
\end{tabular} & -3 \\
\hline TOC 1 & \begin{tabular}{|l|l|}
$01 / 12 / 2013$ \\
\end{tabular} & 0.98 & 1 & \begin{tabular}{|l|}
-0.02 \\
\end{tabular} & -2 \\
\hline TOC 1 & $01 / 13 / 2013$ & 0.96 & 1 & -0.04 & -4 \\
\hline TOC 2 & $01 / 13 / 2013$ & 0.97 & 1 & -0.03 & -3 \\
\hline TOC 1 & $01 / 13 / 2013$ & 0.97 & 1 & \begin{tabular}{|l|}
-0.03 \\
\end{tabular} & -3 \\
\hline TOC 2 & 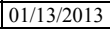 & 0.97 & 1 & -0.03 & -3 \\
\hline
\end{tabular}


SRNL-STI-2013-00139

Revision 0

Table A1. DWPF Laboratory Measurements of Anion and TOC Standards

\begin{tabular}{|c|c|c|c|c|c|c|c|c|c|c|c|}
\hline Analyte & Date & \begin{tabular}{|c|}
$\begin{array}{c}\text { Measurement } \\
\text { (ppm) }\end{array}$ \\
\end{tabular} & $\begin{array}{c}\text { Reference } \\
\text { Value (ppm) }\end{array}$ & diff & $\begin{array}{c}\text { \% rel } \\
\text { diff }\end{array}$ & Analyte & Date & \begin{tabular}{|c|}
$\begin{array}{c}\text { Measurement } \\
\text { (ppm) }\end{array}$ \\
\end{tabular} & $\begin{array}{c}\text { Reference } \\
\text { Value (ppm) }\end{array}$ & diff & $\begin{array}{c}\% \text { rel } \\
\text { diff }\end{array}$ \\
\hline TOC 1 & $01 / 14 / 2013$ & $\begin{array}{r}0.96 \\
\end{array}$ & 1 & \begin{tabular}{|l|}
-0.04 \\
\end{tabular} & -4 & TOC 2 & $12 / 25 / 2012$ & 19.75 & 20 & -0.25 & -1.25 \\
\hline TOC 1 & $01 / 14 / 2013$ & 0.96 & 1 & -0.04 & -4 & \begin{tabular}{|l|} 
TOC 1 \\
\end{tabular} & $01 / 12 / 2013$ & 20.09 & 20 & \begin{tabular}{|l|}
0.09 \\
\end{tabular} & 0.45 \\
\hline TOC 1 & $01 / 14 / 2013$ & 0.96 & 1 & $\mid-0.04$ & -4 & \begin{tabular}{|l|} 
TOC 2 \\
\end{tabular} & $01 / 12 / 2013$ & 20.09 & 20 & 0.09 & 0.45 \\
\hline TOC 2 & $01 / 14 / 2013$ & 0.97 & 1 & \begin{tabular}{|l|}
-0.03 \\
\end{tabular} & -3 & TOC 2 & $01 / 12 / 2013$ & 20.3 & 20 & 0.3 & 1.5 \\
\hline TOC 1 & $01 / 14 / 2013$ & 0.97 & 1 & \begin{tabular}{|l|}
-0.03 \\
\end{tabular} & -3 & \begin{tabular}{|l|} 
TOC 1 \\
\end{tabular} & $01 / 12 / 2013$ & 20.33 & 20 & 0.33 & 1.65 \\
\hline TOC 2 & $01 / 14 / 2013$ & 0.97 & 1 & $\mid-0.03$ & -3 & \begin{tabular}{|l|} 
TOC 1 \\
\end{tabular} & $01 / 13 / 2013$ & 20.27 & 20 & 0.27 & 1.35 \\
\hline TOC 2 & $01 / 14 / 2013$ & 0.98 & 1 & \begin{tabular}{|l|}
-0.02 \\
\end{tabular} & -2 & TOC 2 & $01 / 13 / 2013$ & 20.44 & 20 & 0.44 & 2.2 \\
\hline TOC 2 & $01 / 14 / 2013$ & 0.98 & 1 & -0.02 & -2 & TOC 1 & $01 / 13 / 2013$ & 20.65 & 20 & 0.65 & 3.25 \\
\hline TOC 1 & $01 / 22 / 2013$ & 1.03 & 1 & 0.03 & 3 & TOC 2 & $01 / 13 / 2013$ & 20.73 & 20 & 0.73 & 3.65 \\
\hline TOC 2 & $01 / 22 / 2013$ & 1.06 & 1 & 0.06 & 6 & TOC 1 & $01 / 14 / 2013$ & 20.27 & 20 & 0.27 & 1.35 \\
\hline TOC 1 & $01 / 22 / 2013$ & 1.06 & 1 & 0.06 & 6 & \begin{tabular}{|l|} 
TOC 1 \\
\end{tabular} & $01 / 14 / 2013$ & 20.44 & 20 & 0.44 & 2.2 \\
\hline TOC 2 & $01 / 22 / 2013$ & 1.06 & 1 & 0.06 & 6 & TOC 1 & $01 / 14 / 2013$ & 20.57 & 20 & 0.57 & 2.85 \\
\hline TOC 1 & $01 / 23 / 2013$ & 0.97 & 1 & -0.03 & -3 & \begin{tabular}{|l|} 
TOC 2 \\
\end{tabular} & $01 / 14 / 2013$ & 20.68 & 20 & 0.68 & 3.4 \\
\hline TOC 2 & $01 / 23 / 2013$ & 0.97 & 1 & \begin{tabular}{|l|}
-0.03 \\
\end{tabular} & -3 & TOC 1 & $01 / 14 / 2013$ & 20.72 & 20 & 0.72 & 3.6 \\
\hline TOC 1 & $01 / 23 / 2013$ & 0.97 & 1 & $\mid-0.03$ & -3 & $\begin{array}{l}\text { TOC } 2 \\
\end{array}$ & $01 / 14 / 2013$ & 20.93 & 20 & 0.93 & 4.65 \\
\hline TOC 2 & $01 / 23 / 2013$ & 0.97 & 1 & -0.03 & -3 & TOC 2 & $01 / 14 / 2013$ & 20.94 & 20 & 0.94 & 4.7 \\
\hline TOC 1 & $01 / 26 / 2013$ & 0.99 & 1 & \begin{tabular}{|l|}
-0.01 \\
\end{tabular} & -1 & TOC 2 & $01 / 14 / 2013$ & 21.27 & 20 & \begin{tabular}{|l|}
1.27 \\
\end{tabular} & 6.35 \\
\hline TOC 2 & $01 / 26 / 2013$ & 1 & 1 & 0 & 0 & $\begin{array}{l}\text { TOC } 1 \\
\end{array}$ & $01 / 22 / 2013$ & 19.83 & 20 & \begin{tabular}{|l|}
-0.17 \\
\end{tabular} & -0.85 \\
\hline TOC 1 & $01 / 26 / 2013$ & 1 & 1 & 0 & 0 & TOC 2 & $01 / 22 / 2013$ & 19.86 & 20 & -0.14 & -0.7 \\
\hline TOC 2 & $01 / 26 / 2013$ & 1.02 & 1 & 0.02 & 2 & TOC 1 & $01 / 22 / 2013$ & 19.86 & 20 & \begin{tabular}{|l|}
-0.14 \\
\end{tabular} & -0.7 \\
\hline TOC 1 & $01 / 28 / 2013$ & 0.99 & 1 & $\mid-0.01$ & -1 & $\begin{array}{l}\text { TOC } 2 \\
\end{array}$ & $01 / 22 / 2013$ & 19.99 & 20 & \begin{tabular}{|l|}
-0.01 \\
\end{tabular} & -0.05 \\
\hline TOC 2 & $01 / 28 / 2013$ & 1 & 1 & 0 & 0 & TOC 1 & $01 / 23 / 2013$ & 20.15 & 20 & 0.15 & 0.75 \\
\hline TOC 1 & $01 / 28 / 2013$ & 1.01 & 1 & 0.01 & 1 & TOC 2 & $01 / 23 / 2013$ & 20.26 & 20 & 0.26 & 1.3 \\
\hline TOC 2 & $01 / 28 / 2013$ & 1.02 & 1 & 0.02 & 2 & $\begin{array}{l}\text { TOC } 1 \\
\end{array}$ & $01 / 23 / 2013$ & 20.3 & 20 & \begin{tabular}{|l|}
0.3 \\
\end{tabular} & 1.5 \\
\hline TOC 1 & $02 / 01 / 2013$ & 0.95 & 1 & -0.05 & -5 & TOC 2 & $01 / 23 / 2013$ & 20.44 & 20 & \begin{tabular}{|l|}
0.44 \\
\end{tabular} & 2.2 \\
\hline TOC 2 & $02 / 01 / 2013$ & 0.96 & 1 & \begin{tabular}{|l|}
-0.04 \\
\end{tabular} & -4 & TOC 1 & $01 / 26 / 2013$ & 19.81 & 20 & \begin{tabular}{|l|}
-0.19 \\
\end{tabular} & -0.95 \\
\hline TOC 1 & $02 / 01 / 2013$ & 0.96 & 1 & \begin{tabular}{|c|}
-0.04 \\
\end{tabular} & -4 & TOC 2 & $01 / 26 / 2013$ & 19.83 & 20 & \begin{tabular}{|l|}
-0.17 \\
\end{tabular} & -0.85 \\
\hline TOC 2 & $02 / 01 / 2013$ & 0.96 & 1 & -0.04 & -4 & TOC 1 & $01 / 26 / 2013$ & 20.02 & 20 & \begin{tabular}{|l|}
0.02 \\
\end{tabular} & 0.1 \\
\hline TOC 1 & $02 / 01 / 2013$ & 1 & 1 & 0 & 0 & TOC 2 & $01 / 26 / 2013$ & 20.12 & 20 & \begin{tabular}{|l|}
0.12 \\
\end{tabular} & 0.6 \\
\hline TOC 2 & $02 / 01 / 2013$ & 1.04 & 1 & 0.04 & 4 & \begin{tabular}{|l|} 
TOC 1 \\
\end{tabular} & $01 / 28 / 2013$ & 19.31 & 20 & \begin{tabular}{|l|}
-0.69 \\
\end{tabular} & -3.45 \\
\hline TOC 1 & $02 / 01 / 2013$ & 1.04 & 1 & 0.04 & 4 & $\begin{array}{l}\text { TOC } 2 \\
\end{array}$ & $01 / 28 / 2013$ & 19.32 & 20 & \begin{tabular}{|l|}
-0.68 \\
\end{tabular} & -3.4 \\
\hline TOC 2 & $02 / 01 / 2013$ & 1.05 & 1 & 0.05 & 5 & \begin{tabular}{|l|} 
TOC 1 \\
\end{tabular} & $01 / 28 / 2013$ & 19.65 & 20 & \begin{tabular}{|l|}
-0.35 \\
\end{tabular} & -1.75 \\
\hline TOC 1 & $02 / 09 / 2013$ & 1.03 & 1 & 0.03 & 3 & TOC 2 & $01 / 28 / 2013$ & 19.67 & 20 & -0.33 & -1.65 \\
\hline TOC 2 & $02 / 09 / 2013$ & 1.04 & 1 & 0.04 & 4 & \begin{tabular}{|l|} 
TOC 1 \\
\end{tabular} & $02 / 01 / 2013$ & 19.97 & 20 & \begin{tabular}{|l|}
-0.03 \\
\end{tabular} & -0.15 \\
\hline TOC 1 & $02 / 09 / 2013$ & 1.11 & 1 & 0.11 & 11 & TOC 2 & $02 / 01 / 2013$ & 20.03 & 20 & \begin{tabular}{|l|}
0.03 \\
\end{tabular} & 0.15 \\
\hline TOC 2 & $02 / 09 / 2013$ & 1.12 & 1 & 0.12 & 12 & TOC 1 & $02 / 01 / 2013$ & 20.11 & 20 & 0.11 & 0.55 \\
\hline TOC 1 & $10 / 15 / 2012$ & 18.98 & 20 & -1.02 & -5.1 & TOC 2 & $02 / 01 / 2013$ & 20.21 & 20 & \begin{tabular}{|l|}
0.21 \\
\end{tabular} & 1.05 \\
\hline TOC 2 & $10 / 15 / 2012$ & 19 & 20 & -1 & -5 & \begin{tabular}{|l|} 
TOC 1 \\
\end{tabular} & $02 / 01 / 2013$ & 20.31 & 20 & \begin{tabular}{|l|}
0.31 \\
\end{tabular} & 1.55 \\
\hline TOC 1 & $10 / 15 / 2012$ & 19.23 & 20 & \begin{tabular}{|l|}
-0.77 \\
\end{tabular} & -3.85 & TOC 2 & $02 / 01 / 2013$ & 20.36 & 20 & 0.36 & 1.8 \\
\hline TOC 2 & $10 / 15 / 2012$ & 19.3 & 20 & \begin{tabular}{|l|}
-0.7 \\
\end{tabular} & -3.5 & \begin{tabular}{|l|} 
TOC 1 \\
\end{tabular} & $02 / 01 / 2013$ & 20.43 & 20 & 0.43 & 2.15 \\
\hline TOC 1 & $10 / 24 / 2012$ & 18.89 & 20 & -1.11 & -5.55 & TOC 2 & $02 / 01 / 2013$ & 20.47 & 20 & \begin{tabular}{|l|}
0.47 \\
\end{tabular} & 2.35 \\
\hline TOC 1 & $10 / 24 / 2012$ & 18.92 & 20 & -1.08 & -5.4 & TOC 1 & $02 / 09 / 2013$ & 19.88 & 20 & \begin{tabular}{|l|}
-0.12 \\
\end{tabular} & -0.6 \\
\hline TOC 2 & $10 / 24 / 2012$ & 18.97 & 20 & -1.03 & -5.15 & TOC 2 & $02 / 09 / 2013$ & 19.98 & 20 & -0.02 & -0.1 \\
\hline TOC 2 & $10 / 24 / 2012$ & 19.13 & 20 & -0.87 & -4.35 & \begin{tabular}{|l|} 
TOC 1 \\
\end{tabular} & $02 / 09 / 2013$ & 20.31 & 20 & \begin{tabular}{|l|}
0.31 \\
\end{tabular} & 1.55 \\
\hline TOC 1 & $11 / 21 / 2012$ & 19.74 & 20 & \begin{tabular}{|l|}
-0.26 \\
\end{tabular} & -1.3 & \begin{tabular}{|l|} 
TOC 2 \\
\end{tabular} & $02 / 09 / 2013$ & 20.36 & 20 & 0.36 & 1.8 \\
\hline
\end{tabular}


Exhibit A1. Measurements of Anion and TOC Standards by Date

Analyte=Formate, Analyte - Detail=Formate, Reference Value $(\mathrm{ppm})=2$ Variability Chart for $\%$ rel diff

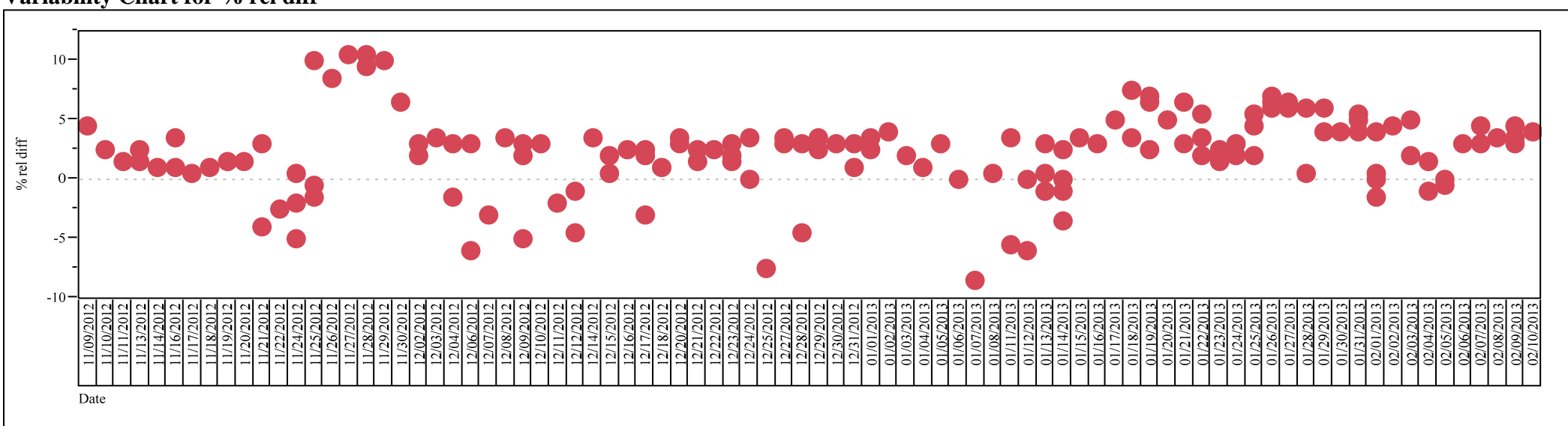

Analyte=Formate, Analyte - Detail=Formate, Reference Value (ppm)=16

Variability Chart for \% rel diff

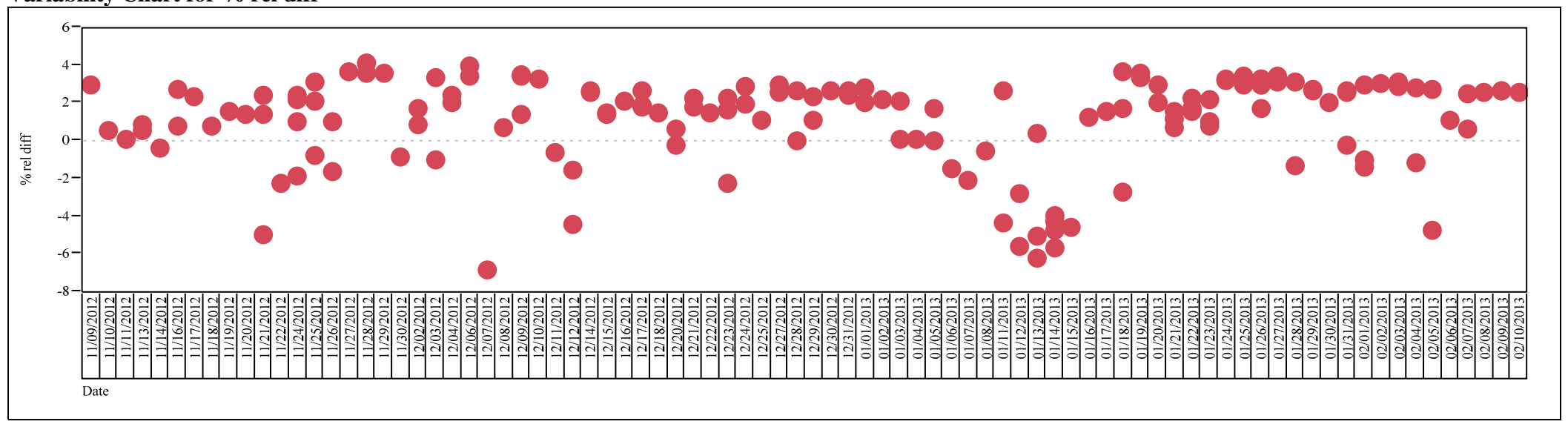


Exhibit A1. Measurements of Anion and TOC Standards by Date

Analyte=Nitrate, Analyte - Detail=Nitrate, Reference Value $(\mathrm{ppm})=2$ Variability Chart for \% rel diff

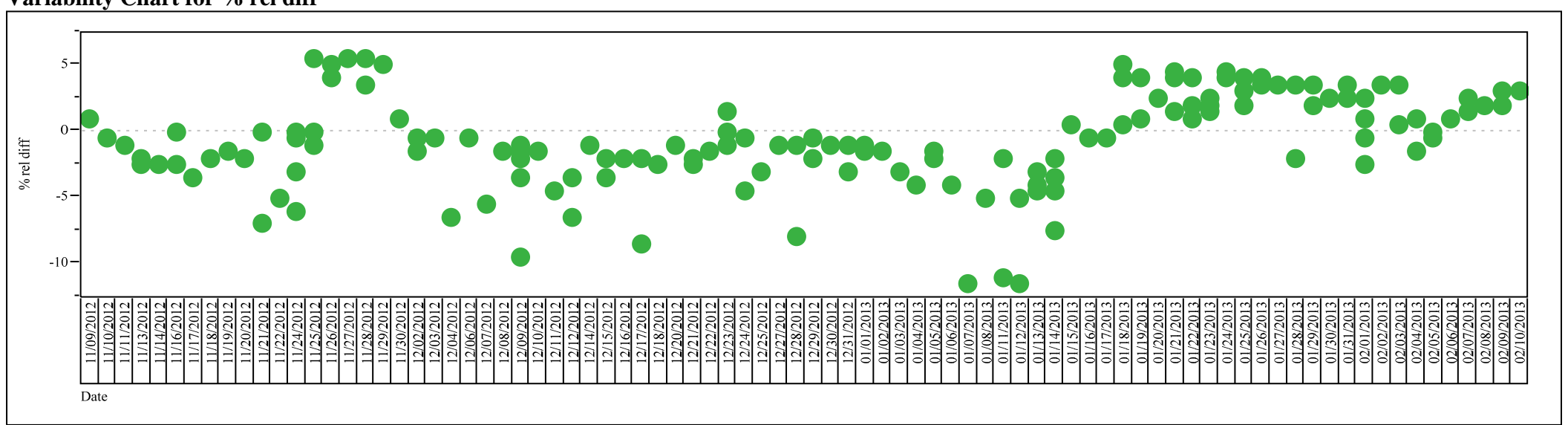

Analyte $=$ Nitrate, Analyte - Detail=Nitrate, Reference Value $(\mathbf{p p m})=16$

Variability Chart for \% rel diff

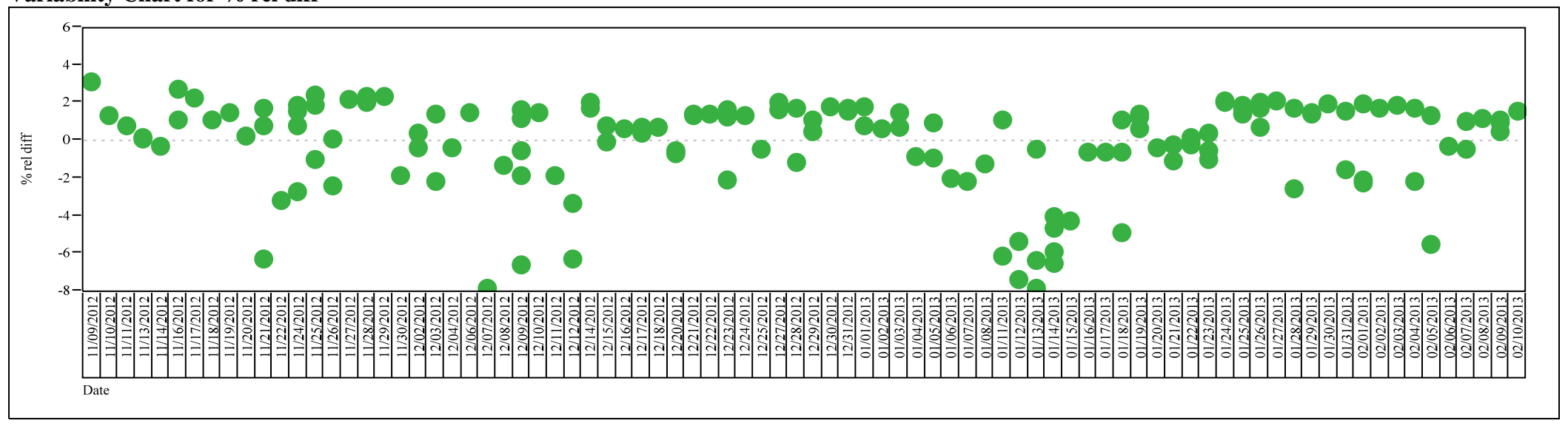




\section{Exhibit A1. Measurements of Anion and TOC Standards by Date}

Analyte $=$ Soluble Oxalate, Analyte - Detail=Soluble Oxalate, Reference Value $(\mathbf{p p m})=2$ Variability Chart for \% rel diff

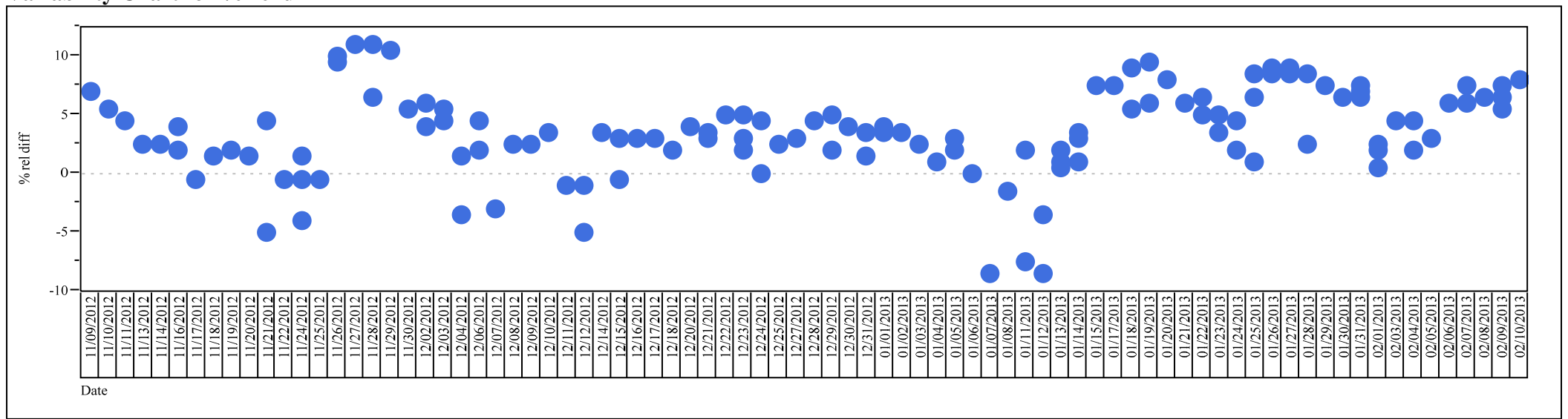

Analyte=Soluble Oxalate, Analyte - Detail=Soluble Oxalate, Reference Value $(\mathbf{p p m})=16$

Variability Chart for \% rel diff

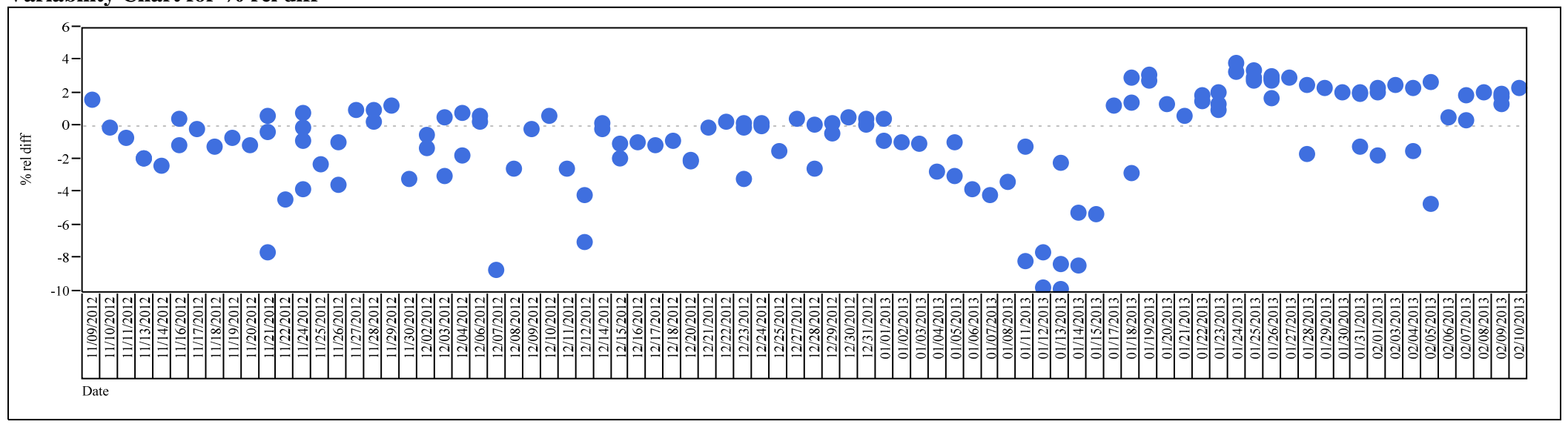




\section{Exhibit A1. Measurements of Anion and TOC Standards by Date}

Analyte=TOC, Analyte - Detail=TOC 1, Reference Value (ppm)=1 Variability Chart for \% rel diff

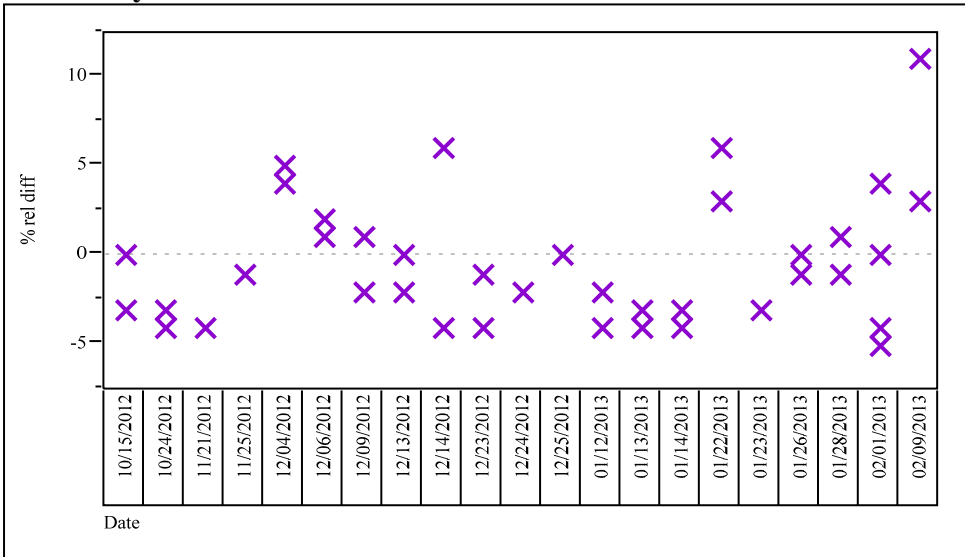

Analyte=TOC, Analyte - Detail=TOC 2

Reference Value (ppm) $=1$

Variability Chart for \% rel diff

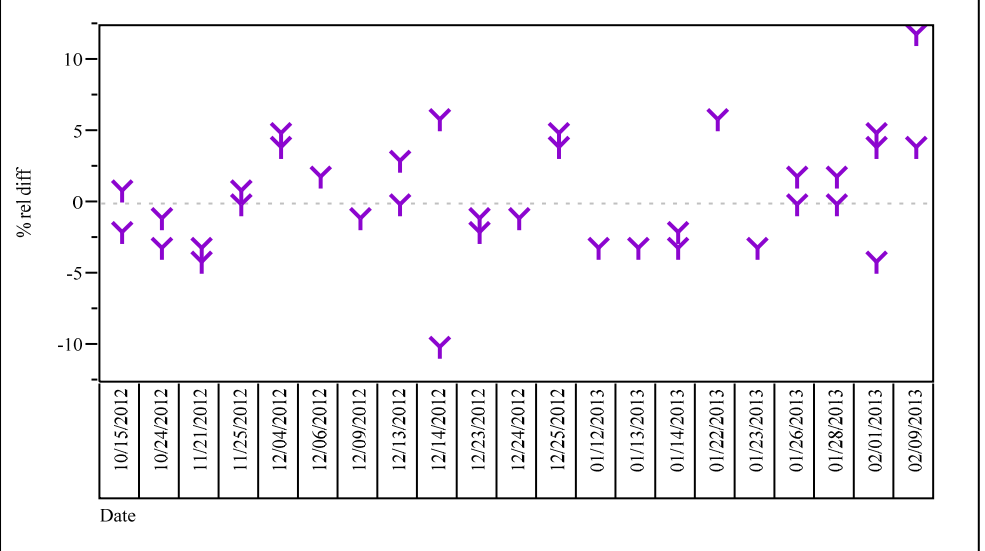

Analyte=TOC, Analyte - Detail=TOC 1, Reference Value (ppm) $=20$ Variability Chart for \% rel diff

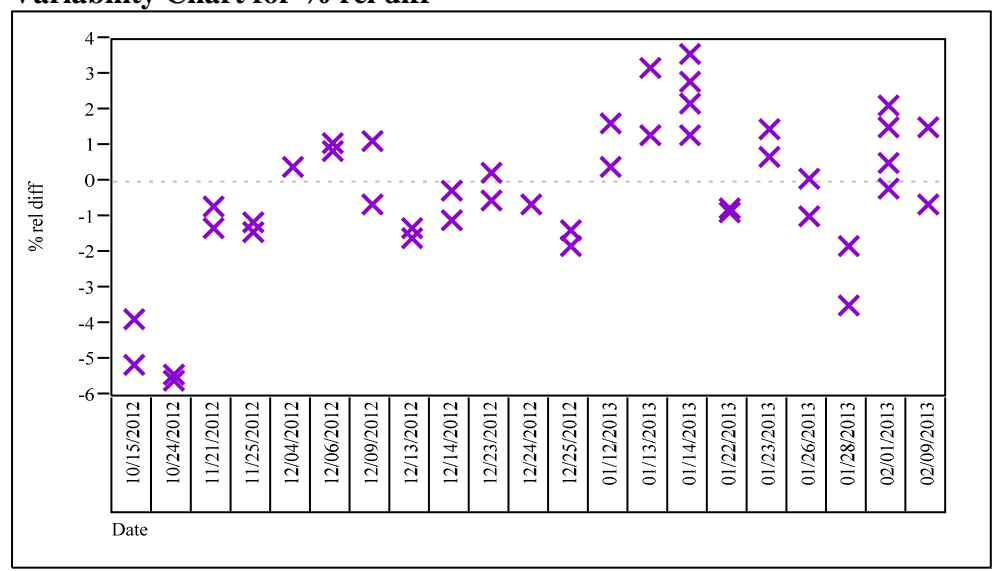

Analyte=TOC, Analyte - Detail=TOC 2,

Reference Value (ppm) $=\mathbf{2 0}$

Variability Chart for \% rel diff

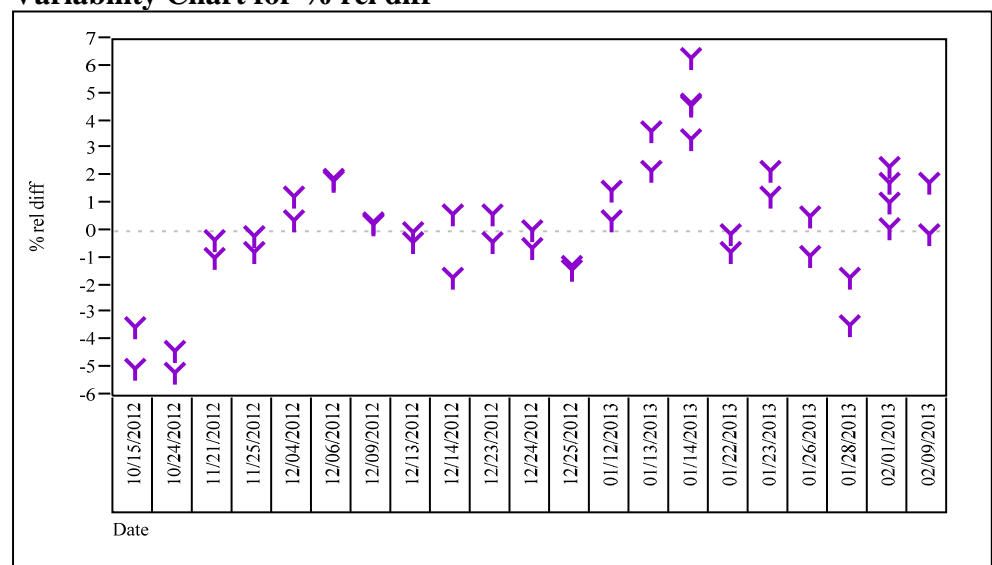


Exhibit A1. Measurements of Anion and TOC Standards by Date

Analyte $=$ Total Oxalate, Analyte - Detail $=$ Total Oxalate, Reference Value (ppm) $=2$ Variability Chart for \% rel diff

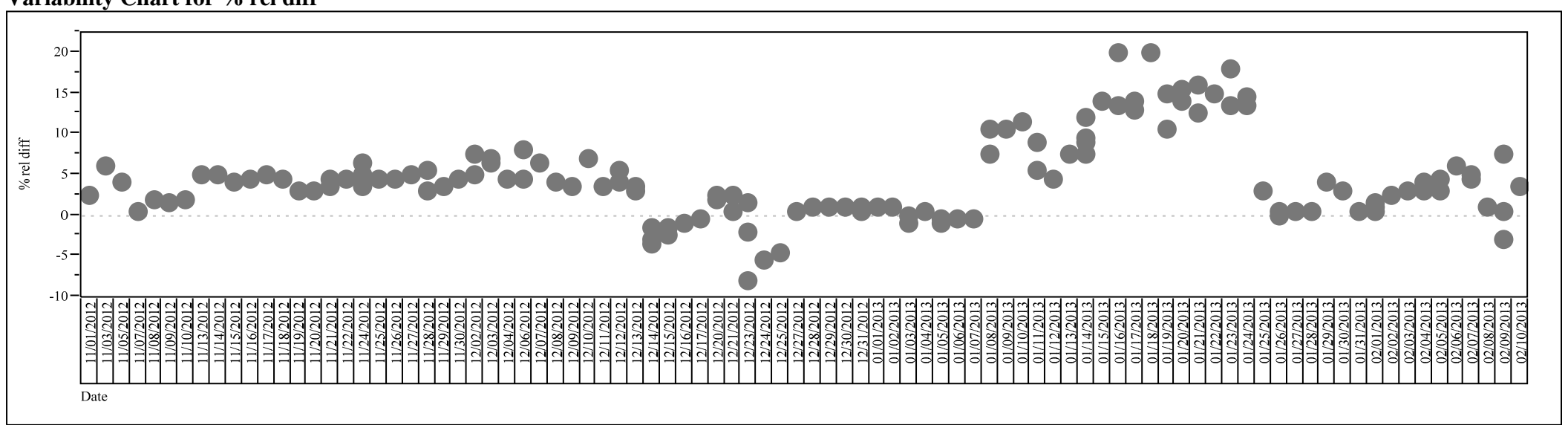

Analyte=Total Oxalate, Analyte - Detail=Total Oxalate, Reference Value (ppm)=16

Variability Chart for \% rel diff

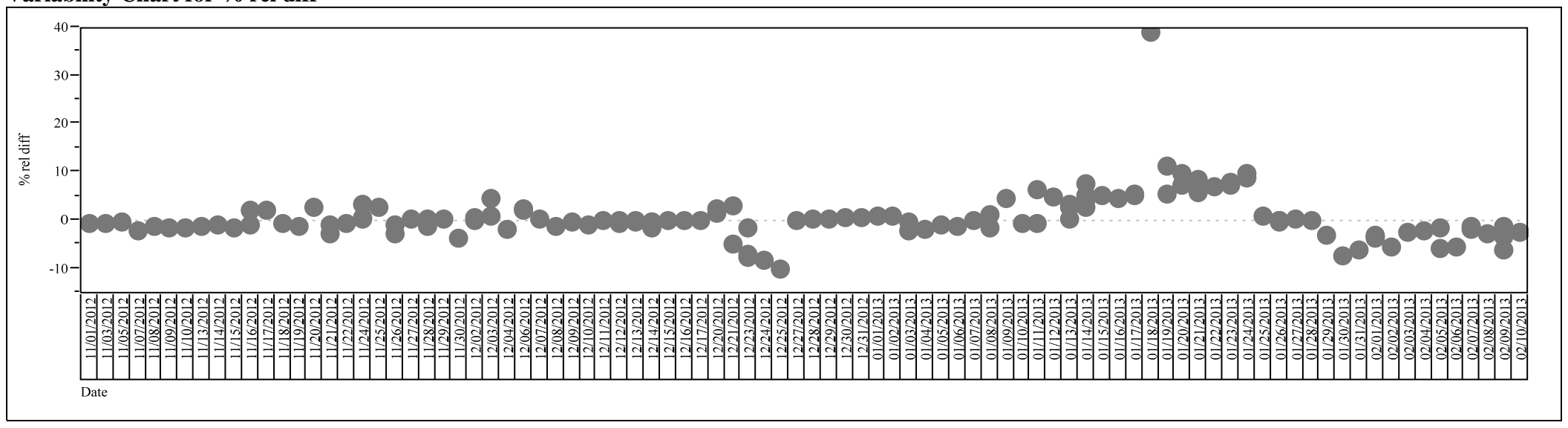


Exhibit A2. Boxplots of the Measurements of Anion and TOC Standards

Variability Gauge Analyte=Formate, Analyte - Detail=Formate, Nominal Value (ppm)=2 Variability Chart for Measurement (ppm)
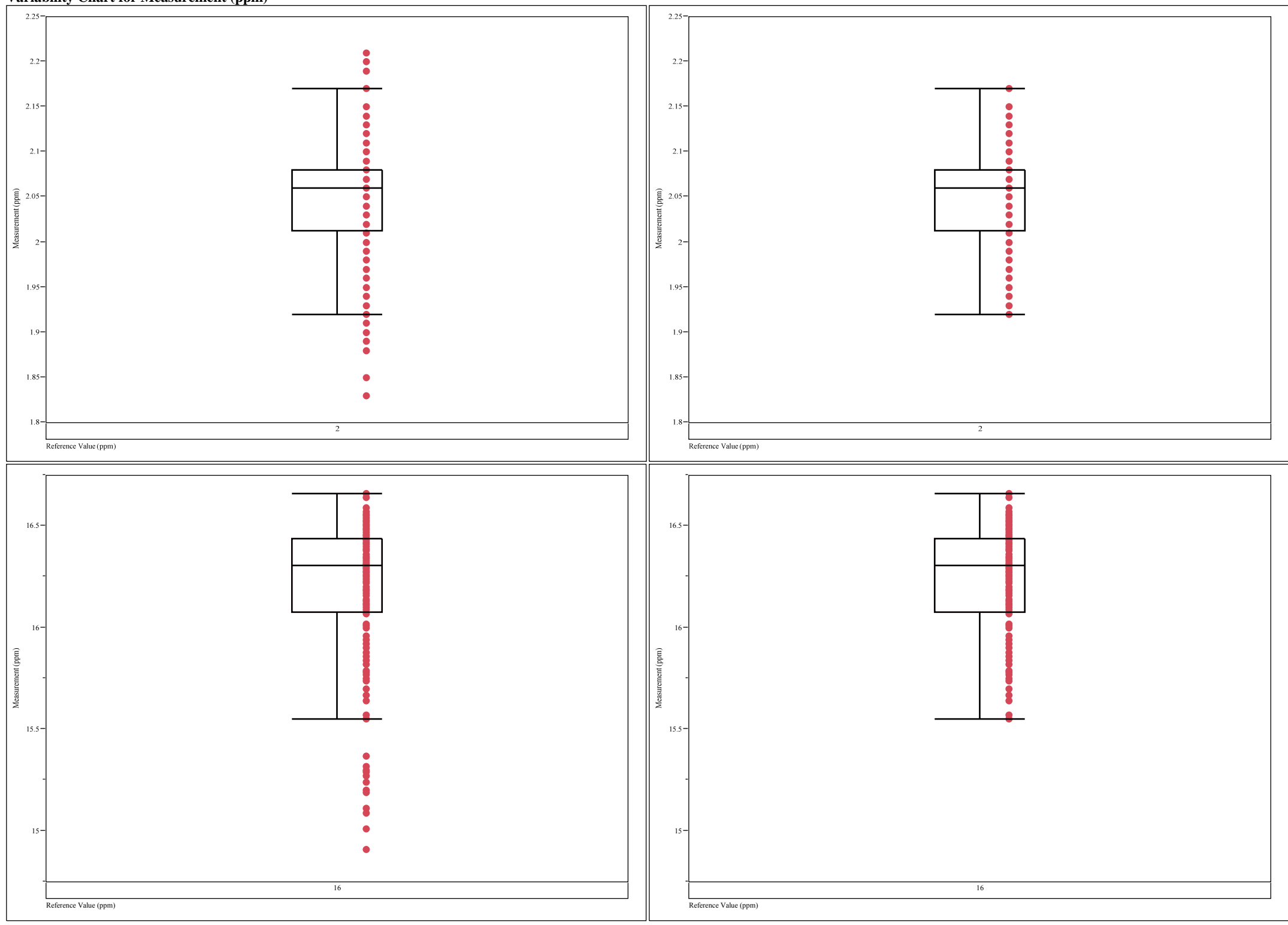
Exhibit A2. Boxplots of the Measurements of Anion and TOC Standards

Variability Gauge Analyte=Nitrate, Analyte - Detail=Nitrate, Nominal Value $(p p m)=2$ Variability Chart for Measurement (ppm)

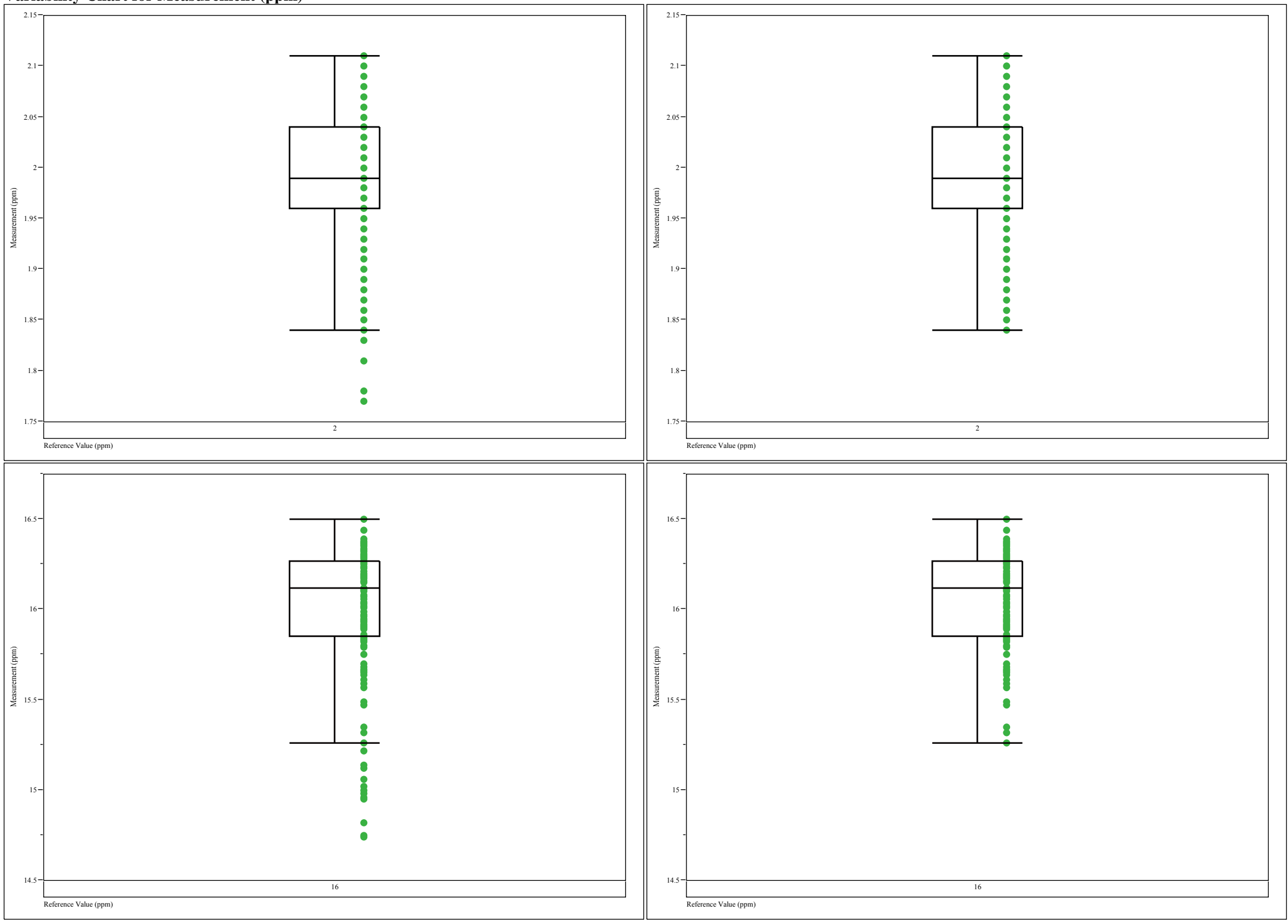




\section{Exhibit A2. Boxplots of the Measurements of Anion and TOC Standards}

Variability Gauge Analyte=Soluble Oxalate, Analyte - Detail=Soluble Oxalate, Nominal Value (ppm)=2

Variability Chart for Measurement (ppm)

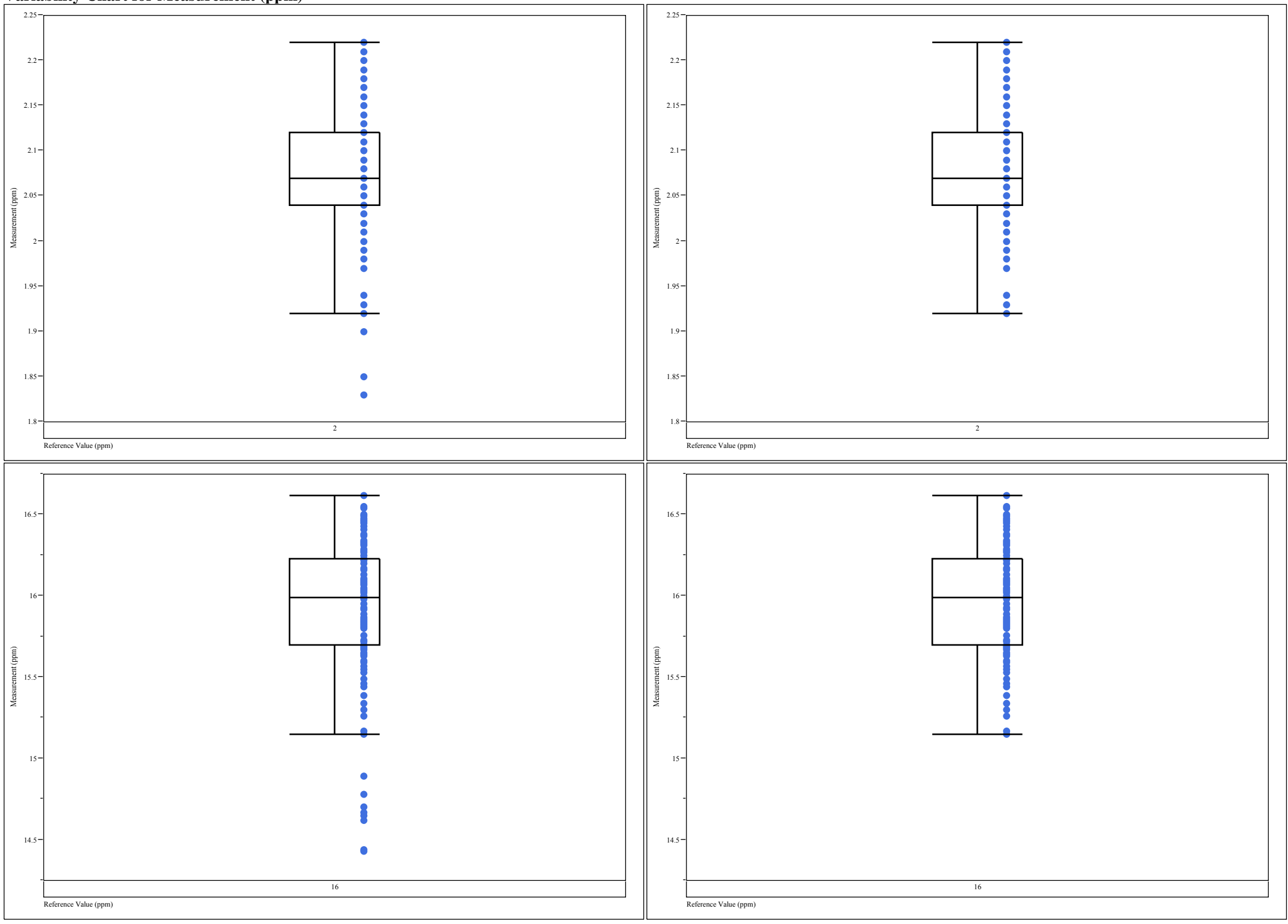


Exhibit A2. Boxplots of the Measurements of Anion and TOC Standards

Variability Gauge Analyte=TOC, Analyte - Detail=TOC 1, Nominal Value (ppm)=1 Variability Chart for Measurement (ppm)

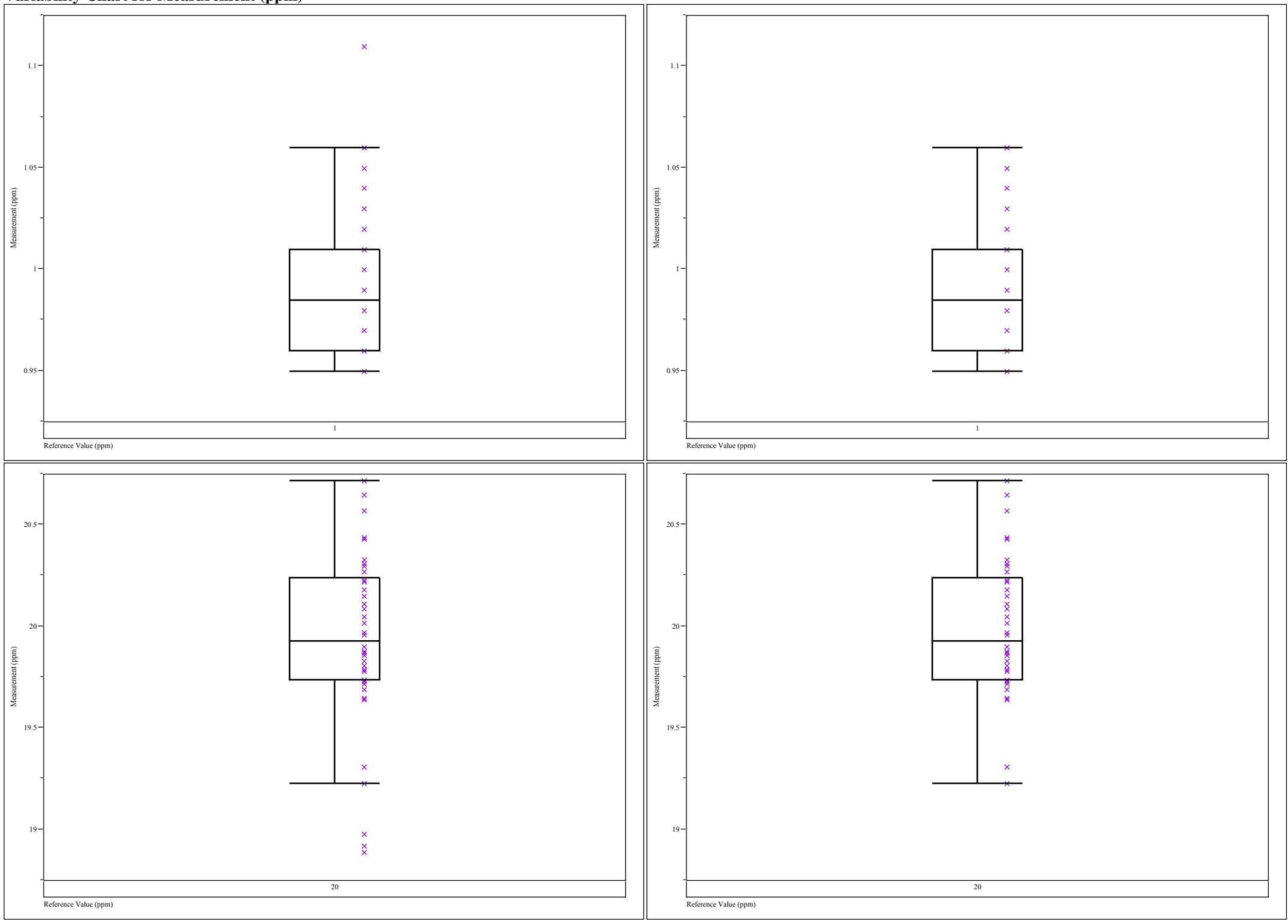


Exhibit A2. Boxplots of the Measurements of Anion and TOC Standards

Variability Gauge Analyte=TOC, Analyte - Detail=TOC 2, Nominal Value (ppm)=1 Variability Chart for Measurement (ppm)

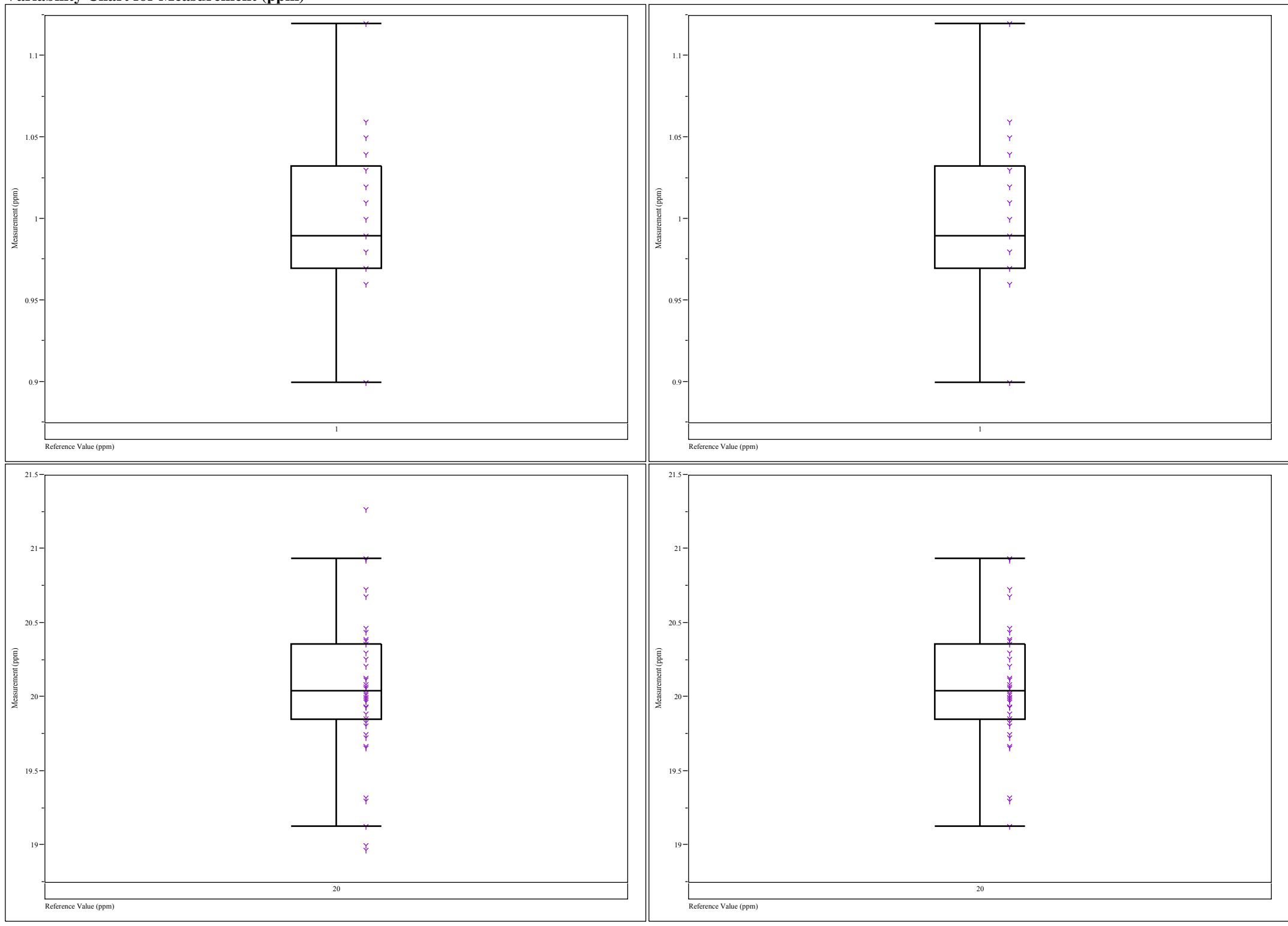




\section{Exhibit A2. Boxplots of the Measurements of Anion and TOC Standards}

Variability Gauge Analyte=Total Oxalate, Analyte - Detail=Total Oxalate, Nominal Value $(\mathrm{ppm})=2$

Variability Chart for Measurement (ppm)
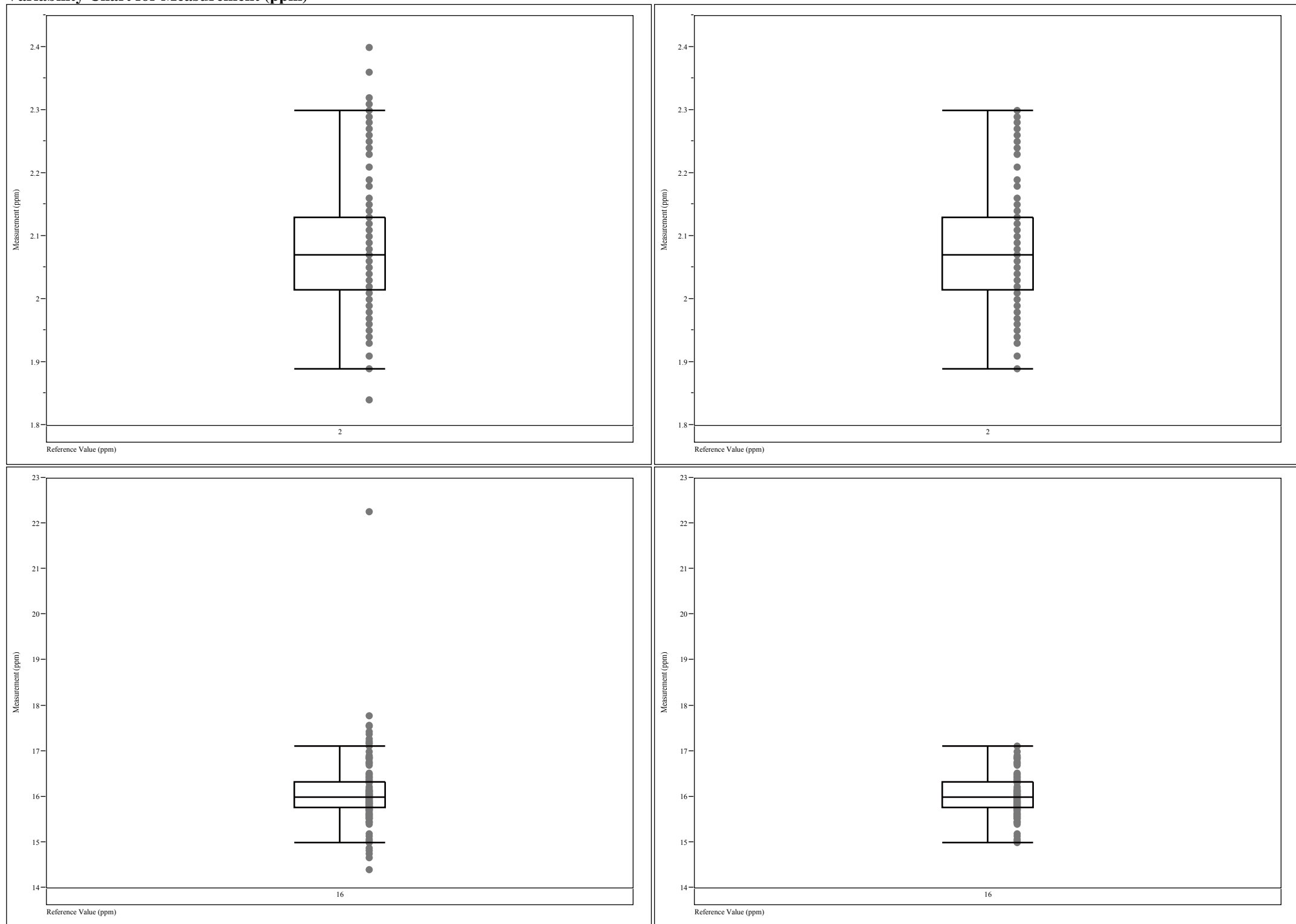


\section{Exhibit A3. Analysis of Variance Results Utilizing All of the Measurements}

Response \% rel diff Analyte=Formate, Reference Value (ppm) $=2$ ummary of Fit

$\begin{array}{lr}\text { RSquare } & 0.658674 \\ \text { RSquare Adj } & 0.658674 \\ \text { Root Mean Square Error } & 2.427439 \\ \text { Mean of Response } & 2.375 \\ \text { Observations (or Sum Wgts) } & 172\end{array}$

Parameter Estimates

Term Estimate Std Error DFDen t Ratio Prob $>|t|$ Lower 95\% Upper 95\% $\begin{array}{llllllll}\text { Intercept } & 2.3245102 & 0.337764 & 80.15 & 6.88<.0001 * & 1.6523578 & 2.9966626\end{array}$

REML Variance Component Estimates

Lower 95\% Upper Pct of Tota $\begin{array}{llllllll}\text { Date } & 1.0348594 & 6.0978682 & 1.5359399 & 3.9321362 & 10.719743 & 50.857\end{array}$ $\begin{array}{lllll}\text { Residual } & 5.8924604 & 0.8760424 & 4.4921277 & 8.0710572\end{array}$ 11.990329

100.000

-2 LogLikelihood $=882.70672337$

Response \% rel diff Analyte=Formate, Reference Value (ppm)=16 Summary of Fit

0.618141

RSquare Adj $\quad 0.618141$

Root Mean Square Error $\quad 1.754986$

$\begin{array}{lr}\text { Mean of Response } & 1.138808 \\ \text { Observations (or Sum Wgts) } & 172\end{array}$

\section{Parameter Estimates}

Term Estimate Std Error DFDen t Ratio Prob $>|\mathbf{t}|$ Lower 95\% Upper 95\% $\begin{array}{llllllll}\text { Intercept } & 1.1333337 & 0.22615 & 83.94 & 5.01<.0001 * & 0.683605 & 1.5830624\end{array}$

REML Variance Component Estimates

Random Effect Var Ratio Var Component Std Error 95\% Lower 95\% Upper Pct of Tota $\begin{array}{lllllll}\text { Date } & 0.8141134 & 2.5074494 & 0.6637224 & 1.5853073 & 4.5560093 & 44.877\end{array}$ $\begin{array}{llllll}\text { Residual } & 3.0799757 & 0.4451162 & 2.3646863 & 4.1789794 & 55.123\end{array}$ $\begin{array}{lllrr}\text { Total } & 5.5874251 & & & \\ & & & & \end{array}$

-2 LogLikelihood $=759.6998011$
Response \% rel diff Analyte $=$ Nitrate, Reference Value $(\mathbf{p p m})=2$ Summary of Fit

$\begin{array}{lr}\text { RSquare } & 0.757416 \\ \text { RSquare Adj } & 0.757416 \\ \text { Root Mean Square Error } & 2.115353 \\ \text { Mean of Response } & -0.49419 \\ \text { Observations (or Sum Wgts) } & 172\end{array}$

Parameter Estimates

Term Estimate Std Error DFDen t Ratio Prob $>|\mathbf{t}|$ Lower 95\% Upper 95\% $\begin{array}{llllllll}\text { Intercept } & -0.707104 & 0.347836 & 81.79 & -2.03 & 0.0453^{*} & -1.399088 & -0.01512\end{array}$

\section{REML Variance Component Estimates}

Random Effect Var Ratio Var Component Std Error 95\% Lower 95\% Upper Pct of Total $\begin{array}{llllllll}\text { Date } & \quad 1.6603838 & 7.4297501 & 1.6033298 & 5.0707968 & 11.931278 & 62.411\end{array}$

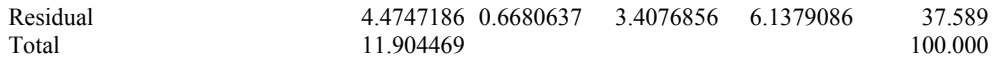

-2 LogLikelihood $=862.2341134$

Response \% rel diff Analyte=Nitrate, Reference Value $(\mathrm{ppm})=16$ Summary of Fit

$\begin{array}{lr}\text { RSquare } & 0.582341 \\ \text { RSquare Adj } & 0.582341 \\ \text { Root Mean Square Error } & 1.836233 \\ \text { Mean of Response } & -0.07086 \\ \text { Observations (or Sum Wgts) } & 172\end{array}$

Parameter Estimates

Prob>|t| Lower 95\% Upper 95\% $\begin{array}{llllllll}\text { Intercept } & -0.023932 & 0.227543 & 84.69 & -0.11 & 0.9165 & -0.476373 & 0.4285092\end{array}$

\section{REML Variance Component Estimates}

Random Effect Var Ratio Var Component Std Error 95\% Lower 95\% Upper Pct of Total $\begin{array}{lllllll}\text { Date } & 0.7068575 & 2.3833469 & 0.6626396 & 1.4765914 & 4.4841349 & 41.413\end{array}$ $\begin{array}{lrrrrr}\text { Residual } & 3.37175 & 0.481439 & 2.5963721 & 4.5568816 & 58.587\end{array}$ $\begin{array}{lrrrr}\text { Total } & 5.7550969 & & & \\ & & & & \end{array}$

-2 LogLikelihood $=768.18030244$ 


\section{Exhibit A3. Analysis of Variance Results Utilizing All of the Measurements}

Response \% rel diff Analyte=Soluble Oxalate, Reference Value $(\mathbf{p p m})=2$ ummary of Fit

$\begin{array}{lr}\text { RSquare } & 0.797124 \\ \text { RSquare Adj } & 0.797124 \\ \text { Root Mean Square Error } & 2.163915 \\ \text { Mean of Response } & 3.581633 \\ \text { Observations (or Sum Wgts) } & 147\end{array}$

Parameter Estimates

Term Estimate Std Error DFDen t Ratio Prob $>|\mathbf{t}|$ Lower 95\% Upper 95\%

$\begin{array}{lllllllll}\text { Intercept } & 3.535159 & 0.385417 & 78.83 & 9.17 & <.0001 * & 2.7679806 & 4.3023374\end{array}$

\section{REML Variance Component Estimates} $\begin{array}{llrlrlrl}\text { Random Effect } & \text { Var Ratio } & \text { Var Component } & \text { Std Error } & \text { 95\% Lower } & \text { 95\% Upper } & \text { Pct of Tota } \\ \text { Date } & 1.9348062 & 9.0597823 & 1.9902095 & 6.1451578 & 14.68848 & 65.926\end{array}$ $\begin{array}{llllll}\text { Residual } & 4.6825271 & 0.8221606 & 3.4120741 & 6.826564 & 34.074\end{array}$ $\begin{array}{llrr}\text { Total } & 13.742309 & 100.000\end{array}$

-2 LogLikelihood

760.21710755

Response \% rel diff Analyte=Soluble Oxalate, Reference Value (ppm) $=16$ Summary of Fit

RSquare

$$
0.731589
$$

Root Mean Square Error $\quad 1.895452$

\begin{tabular}{lr} 
Observations (or Sum Wgts) & -0.59184 \\
\hline
\end{tabular}

\section{Parameter Estimates}

Term Estimate Std Error DFDen t Ratio Prob $>|\mathbf{t}|$ Lower 95\% Upper 95\%

$\begin{array}{lrrrrrrr}\text { Intercept } & -0.693349 & 0.291201 & 84.71 & -2.38 & 0.0195^{*} & -1.272364 & -0.114334\end{array}$

REML Variance Component Estimates

Random Effect Var Ratio Var Component Std Error 95\% Lower 95\% Upper Pct of Tota $\begin{array}{lllllll}\text { Date } & 1.2735472 & 4.5755218 & 1.0758887 & 3.0285176 & 7.7093779 & 56.016\end{array}$ $\begin{array}{lllllll}\text { Residual } & 3.5927383 & 0.5961297 & 2.6603953 & 5.1208071 & 43.984\end{array}$ $\begin{array}{lllll}\text { Total } & 8.16826 & & & \\ & & & & \end{array}$

-2 LogLikelihood $=697.40840142$
Response \% rel diff Analyte=TOC, Reference Value (ppm) $=1$ Summary of Fit

$\begin{array}{lr}\text { RSquare } & 0.59746 \\ \text { RSquare Adj } & 0.59746 \\ \text { Root Mean Square Error } & 2.572658 \\ \text { Mean of Response } & -0.29348 \\ \text { Observations (or Sum Wgts) } & 92\end{array}$

Observations (or Sum Wgts

Parameter Estimates

Term Estimate Std Error DFDen t Ratio Prob $>|t|$ Lower 95\% Upper 95\%

$\begin{array}{llllllll}\text { Intercept } & -0.16464 & 0.652668 & 19.8 & -0.25 & 0.8034 & -1.526949 & 1.1976697\end{array}$

REML Variance Component Estimates

Random Effect Var Ratio Var Component Std Erro $\begin{array}{lllllll}\text { Date } & 1.114229 & 7.3746015 & 2.853044 & 3.9044395 & 18.835186 & 52.701\end{array}$

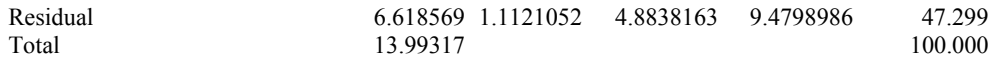

-2 LogLikelihood $=$

469.79796611

Response \% rel diff Analyte=TOC, Reference Value (ppm) $=20$ Summary of Fit

$\begin{array}{lr}\text { RSquare } & 0.882928 \\ \text { RSquare Adj } & 0.882928 \\ \text { Root Mean Square Error } & 0.854231 \\ \text { Mean of Response } & 0.002174 \\ \text { Observations (or Sum Wgts) } & 92\end{array}$

Parameter Estimates

Term Estimate Std Error DFDen t Ratio Prob $>|\mathbf{t}|$ Lower 95\% Upper 95\%

$\begin{array}{llllllll}\text { Intercept } & -0.22151 & 0.446316 & 20.1 & -0.50 & 0.6251 & -1.152215 & 0.709194\end{array}$

REML Variance Component Estimates

Random Effect Var Ratio Var Component Std Error 95\% Lower 95\% Upper Pct of Total $\begin{array}{lllllll}\text { Date } & 5.4946917 & 4.0095367 & 1.3197497 & 2.3031143 & 8.6664422 & 84.603\end{array}$ $\begin{array}{llllll}\text { Residual } & 0.7297109 & 0.1223857 & 0.5387296 & 1.0444233 & 15.397\end{array}$ $\begin{array}{lllll}\text { Total } & 4.7392476 & 1.00000\end{array}$

-2 LogLikelihood $=298.0387362$ 


\section{Exhibit A3. Analysis of Variance Results Utilizing All of the Measurements}

Response \% rel diff Analyte=Total Oxalate, Reference Value (ppm) $=2$

Summary of Fit

$\begin{array}{lr}\text { RSquare } & 0.933962 \\ \text { RSquare Adj } & 0.933962 \\ \text { Root Mean Square Error } & 1.962956 \\ \text { Mean of Response } & 4.417241 \\ \text { Observations (or Sum Wgts) } & 145\end{array}$

$\begin{array}{rr} & 4.417241 \\ \text { Observations (or Sum Wgts) } & 145\end{array}$

Parameter Estimates

Term Estimate Std Error DFDen t Ratio Prob $>|\mathbf{t}|$ Lower 95\% Upper 95\%

$\begin{array}{lllllllll}\text { Intercept } & 4.3860995 & 0.516632 & 90.93 & 8.49 & <.0001 * & 3.3598629 & 5.412336\end{array}$

REML Variance Component Estimates

Random Effect Var Ratio Var Component Std Error 95\% Lower 95\% Upper Pct of Total \begin{tabular}{llllllr} 
Date & 5.4871174 & 21.142935 & 3.5770974 & 15.571163 & 30.365406 & 84.585 \\
\hline
\end{tabular} $\begin{array}{llllll}\text { Residual } & 3.8531953 & 0.7196047 & 2.7564722 & 5.7680065 & 15.415\end{array}$ $\begin{array}{lllll}\text { Total } & 24.99613 & 100.000\end{array}$

-2 LogLikelihood

803.21154614

Response \% rel diff Analyte=Total Oxalate, Reference Value (ppm)=16 Summary of Fit

RSquare Adj $\quad 0.937194$

Root Mean Square Error 1.850139

$\begin{array}{lr}\text { Mean of Response } & 0.568966 \\ \text { Observations (or Sum Wgts) } & 145\end{array}$

\section{Parameter Estimates}

Term Estimate Std Error DFDen t Ratio Prob $>|t|$ Lower 95\% Upper 95\%

$\begin{array}{lrrrrrrr}\text { Intercept } & 0.4386456 & 0.558451 & 85.96 & 0.79 & 0.4343 & -0.671526 & 1.548817\end{array}$

REML Variance Component Estimates

Random Effect Var Ratio Var Component Std Error 95\% Lower 95\% Upper Pct of Total $\begin{array}{llrlrrr}\text { Date } & 7.4496956 & 25.500415 & 4.3244038 & 18.767948 & 36.657437 & 88.165\end{array}$ $\begin{array}{llllll}\text { Residual } & 3.4230144 & 0.6697995 & 2.4132885 & 5.2345602 & 11.835\end{array}$ $\begin{array}{lcrrr}\text { Total } & 28.92343 & & & \\ \end{array}$

-2 LogLikelihood $=810.52221003$ 


\section{Exhibit A4. Analysis of Variance Results Utilizing Screened Measurements}

Response \% rel diff Analyte=Formate, Reference Value (ppm) $=2$ ummary of Fit

$\begin{array}{lr}\text { RSquare } & 0.669789 \\ \text { RSquare Adj } & 0.669789 \\ \text { Root Mean Square Error } & 1.708833 \\ \text { Mean of Response } & 2.612179 \\ \text { Observations (or Sum Wgts) } & 156\end{array}$

Parameter Estimates

Term Estimate Std Error DFDen t Ratio Prob $>|\mathbf{t}|$ Lower 95\% Upper 95\% $\begin{array}{lllllllll}\text { Intercept } & 2.5431886 & 0.242491 & 81.16 & 10.49<.0001 * & 2.0607226 & 3.0256545\end{array}$

\section{REML Variance Component Estimates}

Random Effect Var Ratio Var Component Std Error 95\% Lower 95\% Upper Pct of Total $\begin{array}{lllllll}\text { Date } & 0.9786265 & 2.8576978 & 0.7255778 & 1.8369995 & 5.0497658 & 49.460\end{array}$ $\begin{array}{llllll}\text { Residual } & 2.9201108 & 0.4461764 & 2.210638 & 4.0376856 & 50.540\end{array}$ $\begin{array}{lllr}\text { Total } & 5.7778086 & 100.000\end{array}$

-2 LogLikelihood $=689.97364933$

Response \% rel diff Analyte=Formate, Reference Value (ppm)=16 Summary of Fit

0.203092

RSquare Adj $\quad 0.203092$

Root Mean Square Error $\quad 1.483219$

Mean of Response $\quad 1.689478$

Observations (or Sum Wgts) $\quad 158$

\section{Parameter Estimates}

Term Estimate Std Error DFDen t Ratio Prob $>|t|$ Lower 95\% Upper 95\% $\begin{array}{lllrlrr}\text { Intercept } & 1.6712851 & 0.136973 & 59.67 & 12.20<.0001^{*} & 1.3972674 & 1.9453029\end{array}$

REML Variance Component Estimates

Random Effect Var Ratio Var Component Std Error 95\% Lower 95\% Upper Pct of Tota $\begin{array}{lllllll}\text { Date } & 0.1328641 & 0.2922929 & 0.2881751 & 0.0802065 & 10.569505 & 11.728\end{array}$ $\begin{array}{llllll}\text { Residual } & 2.1999397 & 0.3443145 & 1.6550114 & 3.0679984 & 88.272\end{array}$ $\begin{array}{llll}\text { Total } & 2.4922327 & & \end{array}$

-2 LogLikelihood $=592.26669674$
Response \% rel diff Analyte=Nitrate, Reference Value $(\mathrm{ppm})=2$ Summary of Fit

$\begin{array}{lr}\text { RSquare } & 0.789642 \\ \text { RSquare Adj } & 0.789642 \\ \text { Root Mean Square Error } & 1.754211 \\ \text { Mean of Response } & -0.1976 \\ \text { Observations (or Sum Wgts) } & 167\end{array}$

Term Estimate Std Error DFDen t Ratio Prob $>|t|$ Lower 95\% Upper 95\% $\begin{array}{llllllll}\text { Intercept } & -0.438369 & 0.307911 & 81.63 & -1.42 & 0.1583 & -1.050944 & 0.1742066\end{array}$

REML Variance Component Estimates

Random Effect Var Ratio Var Component Std Error 95\% Lower 95\% Upper Pct of Total \begin{tabular}{lllllll} 
Date & 1.9321424 & 5.9456996 & 1.2407545 & 4.1045202 & 9.3831542 & 65.895 \\
\hline
\end{tabular} $\begin{array}{lrrrrr}\text { Residual } & 3.0772575 & 0.4693084 & 2.3307313 & 4.2521869 & 34.105 \\ \text { Total } & 9.0229571 & & & & 100.000\end{array}$

-2 LogLikelihood $=785.14713632$

Response \% rel diff Analyte=Nitrate, Reference Value $(\mathrm{ppm})=16$ Summary of Fit

$\begin{array}{lr}\text { RSquare } & 0.436081 \\ \text { RSquare Adj } & 0.436081 \\ \text { Root Mean Square Error } & 1.303847 \\ \text { Mean of Response } & 0.521895 \\ \text { Observations (or Sum Wgts) } & 157\end{array}$

Parameter Estimates

Term Estimate Std Error DFDen t Ratio Prob $>|\mathbf{t}|$ Lower 95\% Upper 95\% $\begin{array}{lllrrrrr}\text { Intercept } & 0.4696731 & 0.146369 & 57.75 & 3.21 & 0.0022 * & 0.1766569 & 0.7626893\end{array}$

REML Variance Component Estimates

Random Effect Var Ratio Var Component Std Error 95\% Lower 95\% Upper Pct of Total $\begin{array}{lllllll}\text { Date } & 0.4293125 & 0.7298385 & 0.3625152 & 0.3343649 & 2.6485938 & 30.036\end{array}$ $\begin{array}{llllll}\text { Residual } & 1.700017 & 0.299423 & 1.2376456 & 2.4816236 & 69.964\end{array}$ $\begin{array}{llllll}\text { Total } & 2.4298556 & & & \end{array}$

-2 LogLikelihood $=576.70975117$ 


\section{Exhibit A4. Analysis of Variance Results Utilizing Screened Measurements}

Response \% rel diff Analyte=Soluble Oxalate, Reference Value $(\mathbf{p p m})=2$ ummary of Fit

$\begin{array}{lr}\text { RSquare } & 0.83463 \\ \text { RSquare Adj } & 0.83463 \\ \text { Root Mean Square Error } & 1.713166 \\ \text { Mean of Response } & 3.950704 \\ \text { Observations (or Sum Wgts) } & 142\end{array}$

Parameter Estimates

Term Estimate Std Error DFDen t Ratio Prob $>|\mathbf{t}|$ Lower 95\% Upper 95\% $\begin{array}{lllllllll}\text { Intercept } & 3.8597184 & 0.335376 & 77.26 & 11.51<.0001 * & 3.1919367 & 4.5275\end{array}$

\section{REML Variance Component Estimates}

Upper Pct of Tota $\begin{array}{llllllll}\text { Date } & 2.4162829 & 7.0916436 & 1.5029649 & 4.8700815 & 11.280917 & 70.728\end{array}$ $\begin{array}{llllll}\text { Residual } & 2.9349393 & 0.5370179 & 2.1126688 & 4.3541439 & 29.272\end{array}$

10.026583

-2 LogLikelihood $=683.00153828$

Response \% rel diff Analyte=Soluble Oxalate, Reference Value $(\mathbf{p p m})=16$ Summary of Fit

RSquare Adj $\quad 0.609615$

Root Mean Square Error $\quad 1.561006$

Mean of Response $\quad-0.02418$

Observations (or Sum Wgts) $\quad 137$

Parameter Estimates

Term Estimate Std Error DFDen t Ratio Prob $>|\mathbf{t}|$ Lower 95\% Upper 95\% $\begin{array}{llllllrr}\text { Intercept } & -0.216472 & 0.211321 & 71.83 & -1.02 & 0.3091 & -0.637749 & 0.2048053\end{array}$

REML Variance Component Estimates

Random Effect Var Ratio Var Component Std Error 95\% Lower 95\% Upper Pct of Tota $\begin{array}{lllllll}\text { Date } & 0.7857824 & 1.9147481 & 0.6457306 & 1.0870944 & 4.2337115 & 44.002\end{array}$ $\begin{array}{llllll}\text { Residual } & 2.4367409 & 0.4575394 & 1.7402884 & 3.6564574 & 55.998\end{array}$

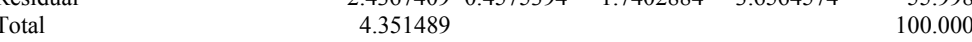

-2 LogLikelihood $=575.92543962$
Response \% rel diff Analyte=TOC, Reference Value (ppm) $=1$ Summary of Fit

$\begin{array}{lr}\text { RSquare } & 0.561156 \\ \text { RSquare Adj } & 0.561156 \\ \text { Root Mean Square Error } & 2.550307 \\ \text { Mean of Response } & -0.41758 \\ \text { Observations (or Sum Wgts) } & 91\end{array}$

Parameter Estimates

Term Estimate Std Error DFDen t Ratio Prob $>|\mathbf{t}|$ Lower 95\% Upper 95\% $\begin{array}{llllllll}\text { Intercept } & -0.241309 & 0.613701 & 19.3 & -0.39 & 0.6985 & -1.524471 & 1.0418526\end{array}$

REML Variance Component Estimates

Random Effect Var Ratio Var Component Std Error 95\% Lower 95\% Upper Pct of Total $\begin{array}{lllllll}\text { Date } & 0.9750435 & 6.3417445 & 2.5604445 & 3.2809814 & 17.044239 & 49.368\end{array}$

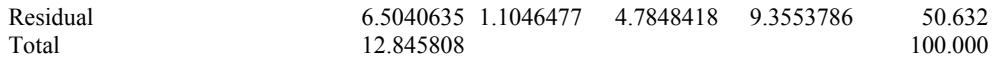

-2 LogLikelihood $=461.10053743$

\section{Response \% rel diff Analyte=TOC, Reference Value (ppm) $=20$} Summary of Fit

$\begin{array}{lr}\text { RSquare } & 0.842579 \\ \text { RSquare Adj } & 0.842579 \\ \text { Root Mean Square Error } & 0.794719 \\ \text { Mean of Response } & 0.23314 \\ \text { Observations (or Sum Wgts) } & 86\end{array}$

Parameter Estimates

Term Estimate Std Error DFDen t Ratio Prob $>|t|$ Lower 95\% Upper 95\%

$\begin{array}{llllllll}\text { Intercept } & -0.13755 & 0.391172 & 18.91 & -0.35 & 0.7290 & -0.956539 & 0.6814389\end{array}$

\section{REML Variance Component Estimates}

Random Effect Var Ratio Var Component Std Error 95\% Lower 95\% Upper Pct of Total $\begin{array}{llllllr}\text { Date } & 4.8052691 & 3.0349036 & 1.0451216 & 1.7055548 & 6.8473579 & 82.774\end{array}$ $\begin{array}{llllll}\text { Residual } & 0.6315783 & 0.1117264 & 0.4592145 & 0.9236298 & 17.226\end{array}$ $\begin{array}{lllll}\text { Total } & 3.6664819 & & 1.226\end{array}$

-2 LogLikelihood $=266.03182726$ 


\section{Exhibit A4. Analysis of Variance Results Utilizing Screened Measurements}

Response \% rel diff Analyte=Total Oxalate, Reference Value (ppm) $=2$

Summary of Fit

$\begin{array}{lr}\text { RSquare } & 0.945955 \\ \text { RSquare Adj } & 0.945955 \\ \text { Root Mean Square Error } & 1.58117 \\ \text { Mean of Response } & 4.021583 \\ \text { Observations (or Sum Wgts) } & 139\end{array}$

Observations (or Sum Wgts) \begin{tabular}{rr}
4.021583 \\
\hline
\end{tabular}

Parameter Estimates

Term Estimate Std Error DFDen t Ratio Prob $>|t|$ Lower 95\% Upper 95\%

$\begin{array}{llllllll}\text { Intercept } & 4.1162088 & 0.460629 & 88.37 & 8.94 & <.0001 * & 3.2008592 & 5.0315584\end{array}$

REML Variance Component Estimates

Random Effect Var Ratio Var Component Std Error 95\% Lower 95\% Upper Pct of Total

$\begin{array}{lllllll}\text { Date } & 6.7831249 & 16.958481 & 2.858398 & 12.502644 & 24.3197 & 87.152\end{array}$

$\begin{array}{llllll}\text { Residual } & 2.5000987 & 0.4969121 & 1.7538078 & 3.851688 & 12.848\end{array}$

$\begin{array}{llll}\text { Total } & 19.45858 & 100.000\end{array}$

-2 LogLikelihood $=$

730.3571031

Response \% rel diff Analyte=Total Oxalate, Reference Value (ppm)=16 Summary of Fit

$\begin{array}{lr}\text { RSquare } & 0.81388 \\ \text { RSquare Adj } & 0.81388 \\ \text { Root Mean Square Error } & 1.564364 \\ \text { Mean of Response } & 0.039904 \\ \text { Observations (or Sum Wgts) } & 130\end{array}$

Parameter Estimates

Term Estimate Std Error DFDen t Ratio Prob $>|\mathbf{t}|$ Lower 95\% Upper 95\%

$\begin{array}{llllllll}\text { Intercept } & -0.014665 & 0.280109 & 81.82 & -0.05 & 0.9584 & -0.57191 & 0.5425\end{array}$

REML Variance Component Estimates

Random Effect Var Ratio Var Component Std Error 95\% Lower 95\% Upper Pct of Total $\begin{array}{lllllll}\text { Date } & 1.9149787 & 4.6864004 & 1.0598033 & 3.1462399 & 7.7207174 & 65.694\end{array}$ $\begin{array}{llllll}\text { Residual } & 2.4472337 & 0.495362 & 1.7065697 & 3.8037416 & 34.306\end{array}$ $\begin{array}{llllr}\text { Total } & 7.1336341 & & & \\ & & & & \end{array}$

-2 LogLikelihood $=594.9843697$ 
SRNL-STI-2013-00139

Revision 0

\section{Exhibit A5. Graphical Comparisons of Choi versus Linear TOC to Nitrate Equations}

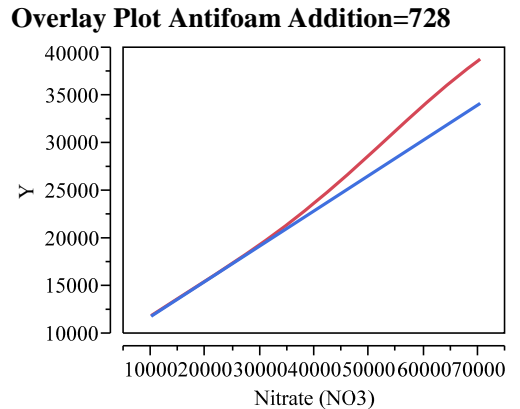

Overlay Plot Antifoam Addition=894

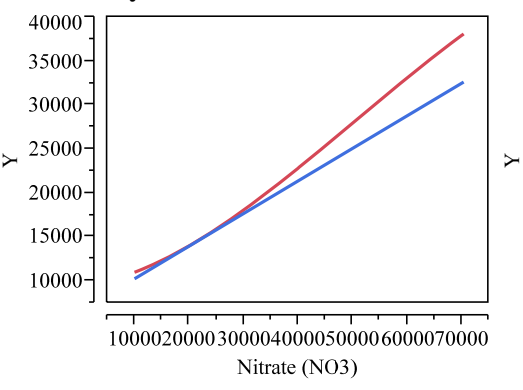

$\mathrm{Y}$ - Choi Equation - Linear Equation

Distributions Antifoam Addition=894 Choi - Linear

Choi - Linear

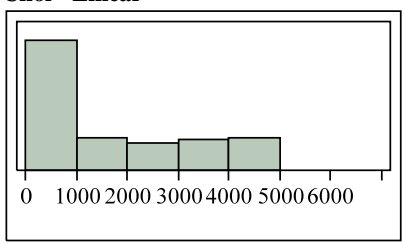

\section{Quantiles}

$100.0 \%$ maximum 4637.78

$99.5 \% \quad 4630.99$

$97.5 \% \quad 4576.59$

$90.0 \% \quad 4194.18$

$75.0 \%$ quartile 2933.73

$50.0 \%$ median 852.435

$25.0 \%$ quartile $\quad 77.31$

$\begin{array}{ll}10.0 \% & 39.8599\end{array}$

$\begin{array}{ll}2.5 \% & 27.0165\end{array}$

$0.5 \% \quad 26.2181$

$0.0 \%$ minimum 26.194

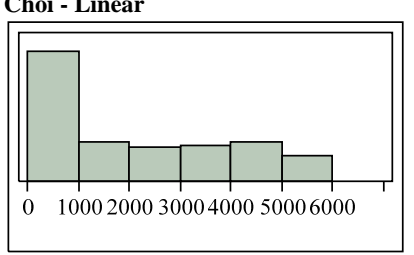

Quantiles

$\begin{array}{llr}100.0 \% & \text { maximum } & 5472.49 \\ 99.5 \% & & 5455.04 \\ 97.5 \% & & 5345.68 \\ 90.0 \% & & 4864.13 \\ 75.0 \% & \text { quartile } & 3656.75 \\ 50.0 \% & \text { median } & 1480.14 \\ 25.0 \% & \text { quartile } & 312.187 \\ 10.0 \% & & 68.862 \\ 2.5 \% & & 25.6118 \\ 0.5 \% & & 22.8802 \\ 0.0 \% & \text { minimum } & 22.7897\end{array}$

\section{Overlay Plot Antifoam Addition=1017}

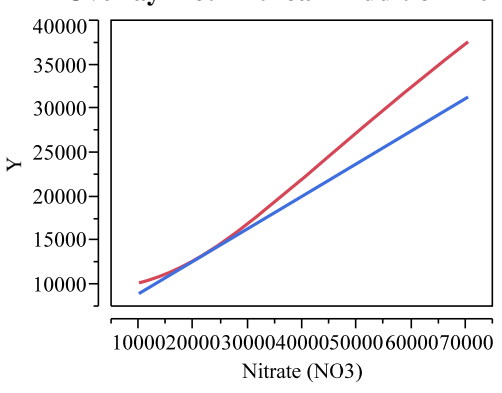

Nitrate (NO3)

Distributions Antifoam Addition=1017 Choi - Linear

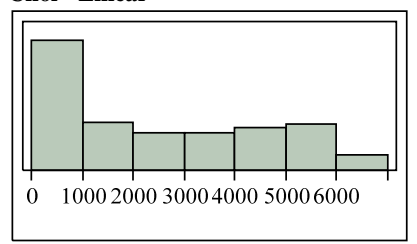

Quantiles

$\begin{array}{llr}100.0 \% & \text { maximum } & 6278.3 \\ 99.5 \% & & 6254.99 \\ 97.5 \% & & 6115.04 \\ 90.0 \% & & 5571.57 \\ 75.0 \% & \text { quartile } & 4349.47 \\ 50.0 \% & \text { median } & 1983.07 \\ 25.0 \% & \text { quartile } & 498.817 \\ 10.0 \% & & 127.222 \\ 2.5 \% & & 60.0232 \\ 0.5 \% & & 56.0008 \\ 0.0 \% & \text { minimum } & 55.8958\end{array}$


SRNL-STI-2013-00139

Revision 0

\section{Appendix B.}

Email for Tommy Edwards

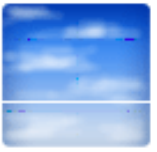

Additional Detail for Uncertainity for MOG Curves

Terri Fellinger to: Tommy Edwards

C. Jonathan Bricker, John laukea, David Sherburne, Damon Click

David Peeler

Tommy.

Based on recent discussions. Al ex has generated three curves for TOC/NO3 vs. NO3 for varying amounts of antifoam and formic acid. Per HLW-DWPF-TTR-2013-0022. DWPF - Engineering is invoking the e-mail clause that states " Any additions or deletions to this Technical Task Request will be communicated via electronic mail from DWPF-E." Based on the three curves, please perform a linearization of each curve minimizing the TOC margin lost between $10.000 \mathrm{ppm}$ and $30.000 \mathrm{ppm}$ nitrate. If you have any questions, please call me.

Thanks.

Terri 


\section{Attachment 1}

SAS CODE

Data Tommy;

Informat MDate Date7.0;

Input Analyte \$ MDate Measurement RefValue;

Difference = Measurement - RefValue;

PctDiff $=100$ * Difference/RefValue;

If Analyte = "TOC1" or Analyte = "TOC2" then Analyte = "TOC";

Cards;

$\{$ data\}

PROC SORT Out=Tomsort; BY Analyte RefValue; RUN;

PROC MIXED ASYCOV NOBOUND DATA=Tomsort ALPHA=0.05;

BY Analyte RefValue;

CLASS MDate;

MODEL PCtDiff $=$ / SOLUTION DDFM=KENWARDROGER;

RANDOM MDate / SOLUTION ; RUN; 
SRNL-STI-2013-00139

Revision 0

\section{All Measurements}

The SAS System

10:41 Thursday, March 14, 201349

Analyte=Formate RefValue $=2$

The Mixed Procedure

Model Information

Data Set

Dependent Variable

Covariance Structure

Estimation Method

Residual Variance Method

Fixed Effects SE Method

Degrees of Freedom Method
WORK. TOMSORT

PctDiff

Variance Components

REML

Profile

Prasad-Rao-Jeske-

Kackar-Harville

Kenward-Roger

\section{Class Level Information}

\begin{tabular}{|c|c|c|c|c|c|c|}
\hline Class & Levels & Values & & & & \\
\hline MDate & 84 & 19306 & 19307 & 19308 & 19310 & 19311 \\
\hline & & 19313 & 19314 & 19315 & 19316 & 19317 \\
\hline & & 19318 & 19319 & 19321 & 19322 & 19323 \\
\hline & & 19324 & 19325 & 19326 & 19327 & 19329 \\
\hline & & 19330 & 19331 & 19333 & 19334 & 19335 \\
\hline & & 19336 & 19337 & 19338 & 19339 & 19341 \\
\hline & & 19342 & 19343 & 19344 & 19345 & 19347 \\
\hline & & 19348 & 19349 & 19350 & 19351 & 19352 \\
\hline & & 19354 & 19355 & 19356 & 19357 & 19358 \\
\hline & & 19359 & 19360 & 19361 & 19362 & 19363 \\
\hline & & 19364 & 19365 & 19366 & 19369 & 19370 \\
\hline & & 19371 & 19372 & 19373 & 19374 & 19375 \\
\hline & & 19376 & 19377 & 19378 & 19379 & 19380 \\
\hline & & 19381 & 19382 & 19383 & 19384 & 19385 \\
\hline & & 19386 & 19387 & 19388 & 19389 & 19390 \\
\hline & & 19391 & 19392 & 19393 & 19394 & 19395 \\
\hline & & 19396 & 19397 & 19398 & 19399 & \\
\hline
\end{tabular}

Dimensions

$\begin{array}{lr}\text { Covariance Parameters } & 2 \\ \text { Columns in X } & 1 \\ \text { Columns in Z } & 84 \\ \text { Subjects } & 1 \\ \text { Max Obs Per Subject } & 172 \\ \text { Observations Used } & 172 \\ \text { Observations Not Used } & 0 \\ \text { Total Observations } & 172\end{array}$

The SAS System

10:41 Thursday, March 14, 201350 
The Mixed Procedure

Iteration History

\begin{tabular}{|c|c|c|c|c|}
\hline Iteration & Evaluations & -2 Res & Log Like & Criterion \\
\hline 0 & 1 & 914 & .17808926 & \\
\hline 1 & 2 & 882 & .70672337 & 0.00000000 \\
\hline & Converge & nce crite & ria met. & \\
\hline & Covariance & Parameter & Estimates & \\
\hline Cov Parm & Estimate & Alpha & Lower & Upper \\
\hline MDate & 6.0979 & 0.05 & 3.0875 & 9.1082 \\
\hline Residual & 5.8925 & 0.05 & 4.4921 & 8.0711 \\
\hline
\end{tabular}

Asymptotic Covariance Matrix of Estimates

$\begin{array}{rlrr}\text { Row } & \text { Cov Parm } & \text { CovP1 } & \text { CovP2 } \\ & & & \\ 1 & \text { MDate } & 2.3591 & -0.4136 \\ 2 & \text { Residual } & -0.4136 & 0.7675\end{array}$

Fit Statistics

$\begin{array}{ll}-2 \text { Res Log Likelihood } & 882.7 \\ \text { AIC (smaller is better) } & 886.7 \\ \text { AICC (smaller is better) } & 886.8 \\ \text { BIC (smaller is better) } & 891.6\end{array}$

Null Model Likelihood Ratio Test

$$
\begin{array}{rrr}
\text { DF } & \text { Chi-Square } & \mathrm{Pr}>\text { ChiSq } \\
1 & 31.47 & <.0001
\end{array}
$$

\begin{tabular}{|c|c|c|c|c|c|}
\hline & & tandard & & & \\
\hline Effect & Estimate & Error & DF & $\mathrm{t}$ Value & $\operatorname{Pr}>|t|$ \\
\hline Intercept & 2.3245 & 0.3378 & 80.1 & 6.88 & $<.0001$ \\
\hline
\end{tabular}

Solution for Fixed Effects 
SRNL-STI-2013-00139

Revision 0

The Mixed Procedure

Solution for Random Effects

\begin{tabular}{|c|c|c|c|c|c|c|}
\hline & & & Std Err & & & \\
\hline Effect & MDate & Estimate & Pred & DF & t Value & $\operatorname{Pr}>|t|$ \\
\hline MDate & 19306 & 1.1064 & 1.7850 & 127 & 0.62 & 0.5365 \\
\hline MDate & 19307 & 0.08925 & 1.7850 & 127 & 0.05 & 0.9602 \\
\hline MDate & 19308 & -0.4193 & 1.7850 & 127 & -0.23 & 0.8147 \\
\hline MDate & 19310 & -0.2188 & 1.4601 & 168 & -0.15 & 0.8811 \\
\hline MDate & 19311 & -0.6736 & 1.7850 & 127 & -0.38 & 0.7065 \\
\hline MDate & 19313 & -0.05024 & 1.4601 & 168 & -0.03 & 0.9726 \\
\hline MDate & 19314 & -0.9279 & 1.7850 & 127 & -0.52 & 0.6041 \\
\hline MDate & 19315 & -0.6736 & 1.7850 & 127 & -0.38 & 0.7065 \\
\hline MDate & 19316 & -0.4193 & 1.7850 & 127 & -0.23 & 0.8147 \\
\hline MDate & 19317 & -0.4193 & 1.7850 & 127 & -0.23 & 0.8147 \\
\hline MDate & 19318 & -1.2539 & 1.2689 & 170 & -0.99 & 0.3245 \\
\hline MDate & 19319 & -2.4536 & 1.7850 & 127 & -1.37 & 0.1717 \\
\hline MDate & 19321 & -3.0804 & 1.1407 & 165 & -2.70 & 0.0076 \\
\hline MDate & 19322 & -0.3620 & 1.1407 & 165 & -0.32 & 0.7513 \\
\hline MDate & 19323 & 4.1637 & 1.4601 & 168 & 2.85 & 0.0049 \\
\hline MDate & 19324 & 4.1578 & 1.7850 & 127 & 2.33 & 0.0214 \\
\hline MDate & 19325 & 5.9316 & 1.2689 & 170 & 4.67 & $<.0001$ \\
\hline MDate & 19326 & 3.9035 & 1.7850 & 127 & 2.19 & 0.0306 \\
\hline MDate & 19327 & 2.1235 & 1.7850 & 127 & 1.19 & 0.2364 \\
\hline MDate & 19329 & 0.1183 & 1.4601 & 168 & 0.08 & 0.9355 \\
\hline MDate & 19330 & 0.7926 & 1.4601 & 168 & 0.54 & 0.5880 \\
\hline MDate & 19331 & -1.0616 & 1.4601 & 168 & -0.73 & 0.4682 \\
\hline MDate & 19333 & -2.5786 & 1.4601 & 168 & -1.77 & 0.0792 \\
\hline MDate & 19334 & -2.7079 & 1.7850 & 127 & -1.52 & 0.1318 \\
\hline MDate & 19335 & 0.5978 & 1.7850 & 127 & 0.33 & 0.7383 \\
\hline MDate & 19336 & -1.7582 & 1.2689 & 170 & -1.39 & 0.1677 \\
\hline MDate & 19337 & 0.3435 & 1.7850 & 127 & 0.19 & 0.8477 \\
\hline MDate & 19338 & -2.1993 & 1.7850 & 127 & -1.23 & 0.2202 \\
\hline MDate & 19339 & -3.4214 & 1.4601 & 168 & -2.34 & 0.0203 \\
\hline MDate & 19341 & 0.7926 & 1.4601 & 168 & 0.54 & 0.5880 \\
\hline MDate & 19342 & -0.6236 & 1.2689 & 170 & -0.49 & 0.6237 \\
\hline MDate & 19343 & 0.08925 & 1.7850 & 127 & 0.05 & 0.9602 \\
\hline MDate & 19344 & -1.3800 & 1.2689 & 170 & -1.09 & 0.2783 \\
\hline MDate & 19345 & -0.6736 & 1.7850 & 127 & -0.38 & 0.7065 \\
\hline MDate & 19347 & 0.6240 & 1.4601 & 168 & 0.43 & 0.6697 \\
\hline MDate & 19348 & -0.2188 & 1.4601 & 168 & -0.15 & 0.8811 \\
\hline MDate & 19349 & 0.08925 & 1.7850 & 127 & 0.05 & 0.9602 \\
\hline MDate & 19350 & 0.04067 & 1.1407 & 165 & 0.04 & 0.9716 \\
\hline MDate & 19351 & -0.3874 & 1.4601 & 168 & -0.27 & 0.7911 \\
\hline MDate & 19352 & -6.6240 & 1.4601 & 168 & -4.54 & $<.0001$ \\
\hline MDate & 19354 & 0.6370 & 1.2689 & 170 & 0.50 & 0.6163 \\
\hline MDate & 19355 & -1.3800 & 1.2689 & 170 & -1.09 & 0.2783 \\
\hline MDate & 19356 & 0.3849 & 1.2689 & 170 & 0.30 & 0.7620 \\
\hline MDate & 19357 & 0.3435 & 1.7850 & 127 & 0.19 & 0.8477 \\
\hline
\end{tabular}

The SAS System

10:41 Thursday, March 14, 201352

Analyte=Formate RefValue=2

The Mixed Procedure 
SRNL-STI-2013-00139

Revision 0

Solution for Random Effects

\begin{tabular}{|c|c|c|c|c|c|c|}
\hline & & & Std Err & & & \\
\hline Effect & MDate & Estimate & Pred & DF & $\mathrm{t}$ Value & $\mathrm{Pr}>|\mathrm{t}|$ \\
\hline MDate & 19358 & -0.2188 & 1.4601 & 168 & -0.15 & 0.8811 \\
\hline MDate & 19359 & 0.4554 & 1.4601 & 168 & 0.31 & 0.7555 \\
\hline MDate & 19360 & 0.8521 & 1.7850 & 127 & 0.48 & 0.6339 \\
\hline MDate & 19361 & -0.2188 & 1.4601 & 168 & -0.15 & 0.8811 \\
\hline MDate & 19362 & -0.6736 & 1.7850 & 127 & -0.38 & 0.7065 \\
\hline MDate & 19363 & 0.4554 & 1.4601 & 168 & 0.31 & 0.7555 \\
\hline MDate & 19364 & -1.1822 & 1.7850 & 127 & -0.66 & 0.5090 \\
\hline MDate & 19365 & -5.5050 & 1.7850 & 127 & -3.08 & 0.0025 \\
\hline MDate & 19366 & -0.9279 & 1.7850 & 127 & -0.52 & 0.6041 \\
\hline MDate & 19369 & -2.2415 & 1.4601 & 168 & -1.54 & 0.1266 \\
\hline MDate & 19370 & -3.5900 & 1.4601 & 168 & -2.46 & 0.0150 \\
\hline MDate & 19371 & -1.1279 & 1.2689 & 170 & -0.89 & 0.3753 \\
\hline MDate & 19372 & -2.8699 & 1.0476 & 160 & -2.74 & 0.0069 \\
\hline MDate & 19373 & 0.5978 & 1.7850 & 127 & 0.33 & 0.7383 \\
\hline MDate & 19374 & 0.3435 & 1.7850 & 127 & 0.19 & 0.8477 \\
\hline MDate & 19375 & 1.3607 & 1.7850 & 127 & 0.76 & 0.4473 \\
\hline MDate & 19376 & 2.9964 & 1.0476 & 160 & 2.86 & 0.0048 \\
\hline MDate & 19377 & 2.2758 & 1.2689 & 170 & 1.79 & 0.0747 \\
\hline MDate & 19378 & 1.3607 & 1.7850 & 127 & 0.76 & 0.4473 \\
\hline MDate & 19379 & 2.2758 & 1.2689 & 170 & 1.79 & 0.0747 \\
\hline MDate & 19380 & 1.0152 & 1.2689 & 170 & 0.80 & 0.4248 \\
\hline MDate & 19381 & -0.3620 & 1.1407 & 165 & -0.32 & 0.7513 \\
\hline MDate & 19382 & 0.1183 & 1.4601 & 168 & 0.08 & 0.9355 \\
\hline MDate & 19383 & 1.2673 & 1.2689 & 170 & 1.00 & 0.3194 \\
\hline MDate & 19384 & 3.3316 & 1.0476 & 160 & 3.18 & 0.0018 \\
\hline MDate & 19385 & 2.6467 & 1.4601 & 168 & 1.81 & 0.0717 \\
\hline MDate & 19386 & 1.3934 & 1.2689 & 170 & 1.10 & 0.2737 \\
\hline MDate & 19387 & 1.8039 & 1.4601 & 168 & 1.24 & 0.2184 \\
\hline MDate & 19388 & 0.8521 & 1.7850 & 127 & 0.48 & 0.6339 \\
\hline MDate & 19389 & 1.8976 & 1.2689 & 170 & 1.50 & 0.1367 \\
\hline MDate & 19390 & -0.9972 & 0.9763 & 157 & -1.02 & 0.3086 \\
\hline MDate & 19391 & 1.1064 & 1.7850 & 127 & 0.62 & 0.5365 \\
\hline MDate & 19392 & 0.5109 & 1.2689 & 170 & 0.40 & 0.6877 \\
\hline MDate & 19393 & -1.3987 & 1.4601 & 168 & -0.96 & 0.3395 \\
\hline MDate & 19394 & -1.7358 & 1.4601 & 168 & -1.19 & 0.2362 \\
\hline MDate & 19395 & 0.3435 & 1.7850 & 127 & 0.19 & 0.8477 \\
\hline MDate & 19396 & 0.9611 & 1.4601 & 168 & 0.66 & 0.5113 \\
\hline MDate & 19397 & 0.5978 & 1.7850 & 127 & 0.33 & 0.7383 \\
\hline MDate & 19398 & 1.0152 & 1.2689 & 170 & 0.80 & 0.4248 \\
\hline MDate & 19399 & 0.8521 & 1.7850 & 127 & 0.48 & 0.6339 \\
\hline
\end{tabular}

The SAS System

10:41 Thursday, March 14, 201353

Analyte=Formate RefValue $=16$

The Mixed Procedure

Model Information

Data Set

WORK. TOMSORT

Dependent Variable

PctDiff 
SRNL-STI-2013-00139

Revision 0

$\begin{array}{ll}\text { Covariance Structure } & \text { Variance Components } \\ \text { Estimation Method } & \text { REML } \\ \text { Residual Variance Method } & \text { Profile } \\ \text { Fixed Effects SE Method } & \text { Prasad-Rao-Jeske- } \\ & \begin{array}{l}\text { Kackar-Harville } \\ \text { Degrees of Freedom Method }\end{array} \\ \end{array}$

Class Level Information

\begin{tabular}{lllllll} 
Class Levels & \multicolumn{3}{l}{ Values } \\
MDate & 84 & 19306 & 19307 & 19308 & 19310 & 19311 \\
& 19313 & 19314 & 19315 & 19316 & 19317 \\
19318 & 19319 & 19321 & 19322 & 19323 \\
19324 & 19325 & 19326 & 19327 & 19329 \\
& 19330 & 19331 & 19333 & 19334 & 19335 \\
19336 & 19337 & 19338 & 19339 & 19341 \\
19342 & 19343 & 19344 & 19345 & 19347 \\
19348 & 19349 & 19350 & 19351 & 19352 \\
19354 & 19355 & 19356 & 19357 & 19358 \\
19359 & 19360 & 19361 & 19362 & 19363 \\
19364 & 19365 & 19366 & 19369 & 19370 \\
19371 & 19372 & 19373 & 19374 & 19375 \\
& 19376 & 19377 & 19378 & 19379 & 19380 \\
19381 & 19382 & 19383 & 19384 & 19385 \\
& 19386 & 19387 & 19388 & 19389 & 19390 \\
19391 & 19392 & 19393 & 19394 & 19395 \\
& 19396 & 19397 & 19398 & 19399 &
\end{tabular}

Dimensions

$\begin{array}{lr}\text { Covariance Parameters } & 2 \\ \text { Columns in X } & 1 \\ \text { Columns in Z } & 84 \\ \text { Subjects } & 1 \\ \text { Max Obs Per Subject } & 172 \\ \text { Observations Used } & 172 \\ \text { Observations Not Used } & 0 \\ \text { Total Observations } & 172\end{array}$

The SAS System 10:41 Thursday, March 14, 201354

Analyte=Formate RefValue $=16$

The Mixed Procedure

Iteration History

$\begin{array}{rrrr}\text { Iteration } & \text { Evaluations } & -2 \text { Res Log Like } & \text { Criterion } \\ 0 & 1 & 790.26409505 & \\ 1 & 2 & 763.50834622 & 0.01131333 \\ 2 & 1 & 760.38165140 & 0.00253792 \\ 3 & 1 & 759.73680056 & 0.00015864 \\ 4 & 1 & 759.69996176 & 0.00000072\end{array}$


SRNL-STI-2013-00139

Revision 0

5

$\begin{array}{ll}759.69980112 & 0.00000000\end{array}$

Convergence criteria met.

Covariance Parameter Estimates

$\begin{array}{lrrrr}\text { Cov Parm } & \text { Estimate } & \text { Alpha } & \text { Lower } & \text { Upper } \\ & & & & \\ \text { MDate } & 2.5074 & 0.05 & 1.2066 & 3.8083 \\ \text { Residual } & 3.0800 & 0.05 & 2.3647 & 4.1790\end{array}$

Asymptotic Covariance Matrix of Estimates

$\begin{array}{rlrr}\text { Row } & \text { Cov Parm } & \text { CovP1 } & \text { CovP2 } \\ & & & \\ 1 & \text { MDate } & 0.4405 & -0.08854 \\ 2 & \text { Residual } & -0.08854 & 0.1981\end{array}$

Fit Statistics

$\begin{array}{ll}\text {-2 Res Log Likelihood } & 759.7 \\ \text { AIC (smaller is better) } & 763.7 \\ \text { AICC (smaller is better) } & 763.8 \\ \text { BIC (smaller is better) } & 768.6\end{array}$

Null Model Likelihood Ratio Test

\begin{tabular}{|c|c|c|}
\hline DF & Chi-Square & $\mathrm{Pr}>\mathrm{ChiSq}$ \\
\hline 1 & 30.56 & $<.0001$ \\
\hline
\end{tabular}

The SAS System

10:41 Thursday, March 14, 201355

Analyte=Formate RefValue $=16$

The Mixed Procedure

Solution for Fixed Effects

$\begin{array}{lrrrrr}\text { Effect } & \text { Estimate } & \begin{array}{r}\text { Standard } \\ \text { Error }\end{array} & \text { DF } & \text { t Value } & \text { Pr }>|t| \\ \text { Intercept } & 1.1333 & 0.2261 & 83.9 & 5.01 & <.0001\end{array}$

Solution for Random Effects

Effect MDate Estimate Pred $t$ Value $\operatorname{Pr}>|\mathrm{t}|$ 
SRNL-STI-2013-00139

Revision 0

\begin{tabular}{|c|c|c|c|c|c|c|}
\hline MDate & 19306 & 0.8096 & 1.2121 & 98.3 & 0.67 & 0.5057 \\
\hline MDate & 19307 & -0.2562 & 1.2121 & 98.3 & -0.21 & 0.8331 \\
\hline MDate & 19308 & -0.4525 & 1.2121 & 98.3 & -0.37 & 0.7097 \\
\hline MDate & 19310 & -0.2568 & 1.0121 & 151 & -0.25 & 0.8000 \\
\hline MDate & 19311 & -0.6769 & 1.2121 & 98.3 & -0.56 & 0.5778 \\
\hline MDate & 19313 & 0.4014 & 1.0121 & 151 & 0.40 & 0.6922 \\
\hline MDate & 19314 & 0.5572 & 1.2121 & 98.3 & 0.46 & 0.6467 \\
\hline MDate & 19315 & -0.1720 & 1.2121 & 98.3 & -0.14 & 0.8874 \\
\hline MDate & 19316 & 0.1926 & 1.2121 & 98.3 & 0.16 & 0.8741 \\
\hline MDate & 19317 & 0.1085 & 1.2121 & 98.3 & 0.09 & 0.9289 \\
\hline MDate & 19318 & -1.0702 & 0.8878 & 169 & -1.21 & 0.2297 \\
\hline MDate & 19319 & -1.5183 & 1.2121 & 98.3 & -1.25 & 0.2133 \\
\hline MDate & 19321 & -0.1498 & 0.8020 & 171 & -0.19 & 0.8520 \\
\hline MDate & 19322 & -0.1498 & 0.8020 & 171 & -0.19 & 0.8520 \\
\hline MDate & 19323 & -0.8957 & 1.0121 & 151 & -0.89 & 0.3775 \\
\hline MDate & 19324 & 1.1462 & 1.2121 & 98.3 & 0.95 & 0.3467 \\
\hline MDate & 19325 & 1.8565 & 0.8878 & 169 & 2.09 & 0.0380 \\
\hline MDate & 19326 & 1.0901 & 1.2121 & 98.3 & 0.90 & 0.3707 \\
\hline MDate & 19327 & -0.9013 & 1.2121 & 98.3 & -0.74 & 0.4589 \\
\hline MDate & 19329 & 0.09164 & 1.0121 & 151 & 0.09 & 0.9280 \\
\hline MDate & 19330 & 0.03356 & 1.0121 & 151 & 0.03 & 0.9736 \\
\hline MDate & 19331 & 0.6918 & 1.0121 & 151 & 0.68 & 0.4953 \\
\hline MDate & 19333 & 1.6017 & 1.0121 & 151 & 1.58 & 0.1156 \\
\hline MDate & 19334 & -3.5658 & 1.2121 & 98.3 & -2.94 & 0.0041 \\
\hline MDate & 19335 & -0.2001 & 1.2121 & 98.3 & -0.17 & 0.8692 \\
\hline MDate & 19336 & 1.1618 & 0.8878 & 169 & 1.31 & 0.1924 \\
\hline MDate & 19337 & 0.9779 & 1.2121 & 98.3 & 0.81 & 0.4217 \\
\hline MDate & 19338 & -0.7891 & 1.2121 & 98.3 & -0.65 & 0.5166 \\
\hline MDate & 19339 & -2.5606 & 1.0121 & 151 & -2.53 & 0.0124 \\
\hline MDate & 19341 & 0.9241 & 1.0121 & 151 & 0.91 & 0.3627 \\
\hline MDate & 19342 & 0.2306 & 0.8878 & 169 & 0.26 & 0.7954 \\
\hline MDate & 19343 & 0.4450 & 1.2121 & 98.3 & 0.37 & 0.7143 \\
\hline MDate & 19344 & 0.7036 & 0.8878 & 169 & 0.79 & 0.4292 \\
\hline MDate & 19345 & 0.1645 & 1.2121 & 98.3 & 0.14 & 0.8923 \\
\hline MDate & 19347 & -0.5860 & 1.0121 & 151 & -0.58 & 0.5635 \\
\hline MDate & 19348 & 0.5563 & 1.0121 & 151 & 0.55 & 0.5834 \\
\hline
\end{tabular}

The SAS System

10:41 Thursday, March 14, 201356

Analyte=Formate RefValue $=16$

The Mixed Procedure

Solution for Random Effects

$\begin{array}{lrrrrrr}\text { Effect } & \text { MDate } & \text { Estimate } & \begin{array}{r}\text { Std Err } \\ \text { Pred }\end{array} & \text { DF } & \text { t Value } & \text { Pr > }|t| \\ \text { MDate } & 19349 & 0.1645 & 1.2121 & 98.3 & 0.14 & 0.8923 \\ \text { MDate } & 19350 & -0.2455 & 0.8020 & 171 & -0.31 & 0.7599 \\ \text { MDate } & 19351 & 0.7886 & 1.0121 & 151 & 0.78 & 0.4371 \\ \text { MDate } & 19352 & -0.00516 & 1.0121 & 151 & -0.01 & 0.9959 \\ \text { MDate } & 19354 & 1.1175 & 0.8878 & 169 & 1.26 & 0.2099 \\ \text { MDate } & 19355 & 0.4671 & 0.8878 & 169 & 0.53 & 0.5995 \\ \text { MDate } & 19356 & 0.5706 & 0.8878 & 169 & 0.64 & 0.5213 \\ \text { MDate } & 19357 & 0.6694 & 1.2121 & 98.3 & 0.55 & 0.5820 \\ \text { MDate } & 19358 & 0.8660 & 1.0121 & 151 & 0.86 & 0.3935\end{array}$


SRNL-STI-2013-00139

Revision 0

\begin{tabular}{|c|c|c|c|c|c|c|}
\hline MDate & 19359 & 0.7886 & 1.0121 & 151 & 0.78 & 0.4371 \\
\hline MDate & 19360 & 0.4731 & 1.2121 & 98.3 & 0.39 & 0.6972 \\
\hline MDate & 19361 & -0.02452 & 1.0121 & 151 & -0.02 & 0.9807 \\
\hline MDate & 19362 & -0.4525 & 1.2121 & 98.3 & -0.37 & 0.7097 \\
\hline MDate & 19363 & -0.1794 & 1.0121 & 151 & -0.18 & 0.8595 \\
\hline MDate & 19364 & -1.1537 & 1.2121 & 98.3 & -0.95 & 0.3435 \\
\hline MDate & 19365 & -1.4342 & 1.2121 & 98.3 & -1.18 & 0.2396 \\
\hline MDate & 19366 & -0.7330 & 1.2121 & 98.3 & -0.60 & 0.5468 \\
\hline MDate & 19369 & -1.2248 & 1.0121 & 151 & -1.21 & 0.2281 \\
\hline MDate & 19370 & -3.2963 & 1.0121 & 151 & -3.26 & 0.0014 \\
\hline MDate & 19371 & -3.3612 & 0.8878 & 169 & -3.79 & 0.0002 \\
\hline MDate & 19372 & -4.8133 & 0.7386 & 169 & -6.52 & $<.0001$ \\
\hline MDate & 19373 & -2.5561 & 1.2121 & 98.3 & -2.11 & 0.0375 \\
\hline MDate & 19374 & 0.05236 & 1.2121 & 98.3 & 0.04 & 0.9656 \\
\hline MDate & 19375 & 0.1926 & 1.2121 & 98.3 & 0.16 & 0.8741 \\
\hline MDate & 19376 & -0.6188 & 0.7386 & 169 & -0.84 & 0.4033 \\
\hline MDate & 19377 & 1.4468 & 1.0121 & 151 & 1.43 & 0.1549 \\
\hline MDate & 19378 & 0.8273 & 1.0121 & 151 & 0.82 & 0.4150 \\
\hline MDate & 19379 & 0.008868 & 0.8878 & 169 & 0.01 & 0.9920 \\
\hline MDate & 19380 & 0.5114 & 0.8878 & 169 & 0.58 & 0.5653 \\
\hline MDate & 19381 & 0.3164 & 0.8020 & 171 & 0.39 & 0.6937 \\
\hline MDate & 19382 & 1.2919 & 1.0121 & 151 & 1.28 & 0.2037 \\
\hline MDate & 19383 & 1.5313 & 0.8878 & 169 & 1.72 & 0.0864 \\
\hline MDate & 19384 & 1.3681 & 0.7386 & 169 & 1.85 & 0.0657 \\
\hline MDate & 19385 & 1.3307 & 1.0121 & 151 & 1.31 & 0.1906 \\
\hline MDate & 19386 & 0.3636 & 0.8878 & 169 & 0.41 & 0.6826 \\
\hline MDate & 19387 & 0.9628 & 1.0121 & 151 & 0.95 & 0.3429 \\
\hline MDate & 19388 & 0.3889 & 1.2121 & 98.3 & 0.32 & 0.7490 \\
\hline MDate & 19389 & 0.3636 & 0.8878 & 169 & 0.41 & 0.6826 \\
\hline MDate & 19390 & 0.3562 & 0.6895 & 166 & 0.52 & 0.6061 \\
\hline MDate & 19391 & 0.8657 & 1.2121 & 98.3 & 0.71 & 0.4768 \\
\hline MDate & 19392 & 1.3540 & 0.8878 & 169 & 1.53 & 0.1291 \\
\hline MDate & 19393 & -0.1794 & 1.0121 & 151 & -0.18 & 0.8595 \\
\hline MDate & 19394 & -1.3216 & 1.0121 & 151 & -1.31 & 0.1936 \\
\hline MDate & 19395 & -0.00374 & 1.2121 & 98.3 & -0.00 & 0.9975 \\
\hline
\end{tabular}

The SAS System

10:41 Thursday, March 14, 201357

Analyte=Formate RefValue $=16$

The Mixed Procedure

Solution for Random Effects

$\begin{array}{lrrrrrr}\text { Effect } & \text { MDate } & \text { Estimate } & \begin{array}{r}\text { Std Err } \\ \text { Pred }\end{array} & \text { DF } & t \text { Value } & \text { Pr > |t } \\ \text { MDate } & 19396 & 0.2659 & 1.0121 & 151 & 0.26 & 0.7931 \\ \text { MDate } & 19397 & 0.6414 & 1.2121 & 98.3 & 0.53 & 0.5979 \\ \text { MDate } & 19398 & 1.0731 & 0.8878 & 169 & 1.21 & 0.2285 \\ \text { MDate } & 19399 & 0.6414 & 1.2121 & 98.3 & 0.53 & 0.5979\end{array}$

The SAS System

10:41 Thursday, March 14, 201358

Analyte $=$ Nitrate RefValue $=2$

The Mixed Procedure 
SRNL-STI-2013-00139

Revision 0

Model Information

\begin{abstract}
Data Set
Dependent Variable Covariance Structure

Estimation Method Residual Variance Method Fixed Effects SE Method

Degrees of Freedom Method
\end{abstract}

WORK. TOMSORT

PctDiff

Variance Components

REML

Profile

Prasad-Rao-Jeske-

Kackar-Harville

Kenward-Roger

Class Level Information

\begin{tabular}{|c|c|c|c|c|c|c|}
\hline Class & Levels & Values & & & & \\
\hline MDate & 84 & 19306 & 19307 & 19308 & 19310 & 19311 \\
\hline & & 19313 & 19314 & 19315 & 19316 & 19317 \\
\hline & & 19318 & 19319 & 19321 & 19322 & 19323 \\
\hline & & 19324 & 19325 & 19326 & 19327 & 19329 \\
\hline & & 19330 & 19331 & 19333 & 19334 & 19335 \\
\hline & & 19336 & 19337 & 19338 & 19339 & 19341 \\
\hline & & 19342 & 19343 & 19344 & 19345 & 19347 \\
\hline & & 19348 & 19349 & 19350 & 19351 & 19352 \\
\hline & & 19354 & 19355 & 19356 & 19357 & 19358 \\
\hline & & 19359 & 19360 & 19361 & 19362 & 19363 \\
\hline & & 19364 & 19365 & 19366 & 19369 & 19370 \\
\hline & & 19371 & 19372 & 19373 & 19374 & 19375 \\
\hline & & 19376 & 19377 & 19378 & 19379 & 19380 \\
\hline & & 19381 & 19382 & 19383 & 19384 & 19385 \\
\hline & & 19386 & 19387 & 19388 & 19389 & 19390 \\
\hline & & 19391 & 19392 & 19393 & 19394 & 19395 \\
\hline & & 19396 & 19397 & 19398 & 19399 & \\
\hline
\end{tabular}

Dimensions

Covariance Parameters

Columns in $X$

Columns in $Z$

Subjects

Max Obs Per Subject

Observations Used

Observations Not Used

Total Observations
2
1
84
1
172
172
0
172

10:41 Thursday, March 14, 2013

Analyte $=$ Nitrate RefValue $=2$

The Mixed Procedure

Iteration History

Iteration Evaluations -2 Res Log Like Criterion 
SRNL-STI-2013-00139

Revision 0

$\begin{array}{llll}0 & 1 & 913.79436280 & \\ 1 & 2 & 862.23505060 & 0.00000340 \\ 2 & 1 & 862.23411352 & 0.00000000\end{array}$

Convergence criteria met.

Covariance Parameter Estimates

$\begin{array}{lrrrr}\text { Cov Parm } & \text { Estimate } & \text { Alpha } & \text { Lower } & \text { Upper } \\ & & & & \\ \text { MDate } & 7.4293 & 0.05 & 4.2871 & 10.5715 \\ \text { Residual } & 4.4749 & 0.05 & 3.4078 & 6.1381\end{array}$

Asymptotic Covariance Matrix of Estimates

$\begin{array}{rlrr}\text { Row } & \text { Cov Parm } & \text { CovP1 } & \text { CovP2 } \\ & & & \\ 1 & \text { MDate } & 2.5703 & -0.2501 \\ 2 & \text { Residual } & -0.2501 & 0.4463\end{array}$

Fit Statistics

$\begin{array}{ll}\text {-2 Res Log Likelihood } & 862.2 \\ \text { AIC (smaller is better) } & 866.2 \\ \text { AICC (smaller is better) } & 866.3 \\ \text { BIC (smaller is better) } & 871.1\end{array}$

Null Model Likelihood Ratio Test

DF Chi-Square $\quad \mathrm{Pr}>$ ChiSq

$1 \quad 51.56 \quad<.0001$

The SAS System 10:41 Thursday, March 14, 201360

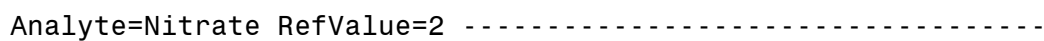

The Mixed Procedure

Solution for Fixed Effects

$\begin{array}{lrrrrr}\text { Effect } & \text { Estimate } & \begin{array}{r}\text { Standard } \\ \text { Error }\end{array} & \text { DF } & t \text { Value } & \operatorname{Pr}>|t| \\ \text { Intercept } & -0.7071 & 0.3478 & 81.8 & -2.03 & 0.0453\end{array}$

Solution for Random Effects

Std Err 
SRNL-STI-2013-00139

Revision 0

\begin{tabular}{|c|c|c|c|c|c|c|}
\hline Effect & MDate & Estimate & Pred & DF & t Value & $\operatorname{Pr}>|t|$ \\
\hline MDate & 19306 & 1.0654 & 1.7170 & 169 & 0.62 & 0.5358 \\
\hline MDate & 19307 & 0.1292 & 1.7170 & 169 & 0.08 & 0.9401 \\
\hline MDate & 19308 & -0.1828 & 1.7170 & 169 & -0.11 & 0.9153 \\
\hline MDate & 19310 & -1.1858 & 1.3571 & 163 & -0.87 & 0.3836 \\
\hline MDate & 19311 & -1.1189 & 1.7170 & 169 & -0.65 & 0.5155 \\
\hline MDate & 19313 & -0.4172 & 1.3571 & 163 & -0.31 & 0.7589 \\
\hline MDate & 19314 & -1.7430 & 1.7170 & 169 & -1.02 & 0.3115 \\
\hline MDate & 19315 & -0.8069 & 1.7170 & 169 & -0.47 & 0.6390 \\
\hline MDate & 19316 & -0.4948 & 1.7170 & 169 & -0.29 & 0.7735 \\
\hline MDate & 19317 & -0.8069 & 1.7170 & 169 & -0.47 & 0.6390 \\
\hline MDate & 19318 & -1.3543 & 1.1641 & 152 & -1.16 & 0.2465 \\
\hline MDate & 19319 & -2.6792 & 1.7170 & 169 & -1.56 & 0.1205 \\
\hline MDate & 19321 & -1.4496 & 1.0404 & 147 & -1.39 & 0.1656 \\
\hline MDate & 19322 & 1.5923 & 1.0404 & 147 & 1.53 & 0.1280 \\
\hline MDate & 19323 & 4.0019 & 1.3571 & 163 & 2.95 & 0.0037 \\
\hline MDate & 19324 & 3.8738 & 1.7170 & 169 & 2.26 & 0.0253 \\
\hline MDate & 19325 & 4.6140 & 1.1641 & 152 & 3.96 & 0.0001 \\
\hline MDate & 19326 & 3.5618 & 1.7170 & 169 & 2.07 & 0.0396 \\
\hline MDate & 19327 & 1.0654 & 1.7170 & 169 & 0.62 & 0.5358 \\
\hline MDate & 19329 & -0.2251 & 1.3571 & 163 & -0.17 & 0.8685 \\
\hline MDate & 19330 & 0.1592 & 1.3571 & 163 & 0.12 & 0.9068 \\
\hline MDate & 19331 & -3.6153 & 1.7170 & 169 & -2.11 & 0.0367 \\
\hline MDate & 19333 & 0.1592 & 1.3571 & 163 & 0.12 & 0.9068 \\
\hline MDate & 19334 & -2.9912 & 1.7170 & 169 & -1.74 & 0.0833 \\
\hline MDate & 19335 & -0.4948 & 1.7170 & 169 & -0.29 & 0.7735 \\
\hline MDate & 19336 & -2.4926 & 0.9531 & 146 & -2.62 & 0.0099 \\
\hline MDate & 19337 & -0.4948 & 1.7170 & 169 & -0.29 & 0.7735 \\
\hline MDate & 19338 & -2.3671 & 1.7170 & 169 & -1.38 & 0.1698 \\
\hline MDate & 19339 & -3.2993 & 1.3571 & 163 & -2.43 & 0.0161 \\
\hline MDate & 19341 & -0.2251 & 1.3571 & 163 & -0.17 & 0.8685 \\
\hline MDate & 19342 & -1.4931 & 1.1641 & 152 & -1.28 & 0.2016 \\
\hline MDate & 19343 & -0.8069 & 1.7170 & 169 & -0.47 & 0.6390 \\
\hline MDate & 19344 & -2.8811 & 1.1641 & 152 & -2.47 & 0.0144 \\
\hline MDate & 19345 & -1.1189 & 1.7170 & 169 & -0.65 & 0.5155 \\
\hline MDate & 19347 & -0.2251 & 1.3571 & 163 & -0.17 & 0.8685 \\
\hline MDate & 19348 & -1.1858 & 1.3571 & 163 & -0.87 & 0.3836 \\
\hline
\end{tabular}

The SAS System

10:41 Thursday, March 14, 201361

Analyte=Nitrate RefValue=2

The Mixed Procedure

Solution for Random Effects

$\begin{array}{lrrrrrr}\text { Effect } & \text { MDate } & \text { Estimate } & \begin{array}{c}\text { Std Err } \\ \text { Pred }\end{array} & \text { DF } & \text { t Value } & \text { Pr }>|t| \\ \text { MDate } & 19349 & -0.4948 & 1.7170 & 169 & -0.29 & 0.7735 \\ \text { MDate } & 19350 & 0.5059 & 1.0404 & 147 & 0.49 & 0.6275 \\ \text { MDate } & 19351 & -1.3779 & 1.3571 & 163 & -1.02 & 0.3115 \\ \text { MDate } & 19352 & -1.7622 & 1.3571 & 163 & -1.30 & 0.1960 \\ \text { MDate } & 19354 & -0.2439 & 1.1641 & 152 & -0.21 & 0.8343 \\ \text { MDate } & 19355 & -2.1871 & 1.1641 & 152 & -1.88 & 0.0622 \\ \text { MDate } & 19356 & -0.6603 & 1.1641 & 152 & -0.57 & 0.5714\end{array}$


SRNL-STI-2013-00139

Revision 0

\begin{tabular}{|c|c|c|c|c|c|c|}
\hline MDate & 19357 & -0.1828 & 1.7170 & 169 & -0.11 & 0.9153 \\
\hline MDate & 19358 & -0.9936 & 1.3571 & 163 & -0.73 & 0.4651 \\
\hline MDate & 19359 & -0.4172 & 1.3571 & 163 & -0.31 & 0.7589 \\
\hline MDate & 19360 & -0.4948 & 1.7170 & 169 & -0.29 & 0.7735 \\
\hline MDate & 19361 & -1.7622 & 1.3571 & 163 & -1.30 & 0.1960 \\
\hline MDate & 19362 & -2.0551 & 1.7170 & 169 & -1.20 & 0.2330 \\
\hline MDate & 19363 & -0.8015 & 1.3571 & 163 & -0.59 & 0.5556 \\
\hline MDate & 19364 & -2.0551 & 1.7170 & 169 & -1.20 & 0.2330 \\
\hline MDate & 19365 & -6.7358 & 1.7170 & 169 & -3.92 & 0.0001 \\
\hline MDate & 19366 & -2.6792 & 1.7170 & 169 & -1.56 & 0.1205 \\
\hline MDate & 19369 & -4.4521 & 1.3571 & 163 & -3.28 & 0.0013 \\
\hline MDate & 19370 & -5.7970 & 1.3571 & 163 & -4.27 & $<.0001$ \\
\hline MDate & 19371 & -2.6035 & 1.1641 & 152 & -2.24 & 0.0268 \\
\hline MDate & 19372 & -3.8314 & 0.9531 & 146 & -4.02 & $<.0001$ \\
\hline MDate & 19373 & 0.7533 & 1.7170 & 169 & 0.44 & 0.6614 \\
\hline MDate & 19374 & 0.1292 & 1.7170 & 169 & 0.08 & 0.9401 \\
\hline MDate & 19375 & 0.1292 & 1.7170 & 169 & 0.08 & 0.9401 \\
\hline MDate & 19376 & 3.1300 & 0.9531 & 146 & 3.28 & 0.0013 \\
\hline MDate & 19377 & 3.0873 & 1.1641 & 152 & 2.65 & 0.0088 \\
\hline MDate & 19378 & 2.0015 & 1.7170 & 169 & 1.17 & 0.2454 \\
\hline MDate & 19379 & 3.3649 & 1.1641 & 152 & 2.89 & 0.0044 \\
\hline MDate & 19380 & 2.5321 & 1.1641 & 152 & 2.18 & 0.0312 \\
\hline MDate & 19381 & 2.2442 & 1.0404 & 147 & 2.16 & 0.0326 \\
\hline MDate & 19382 & 3.8097 & 1.3571 & 163 & 2.81 & 0.0056 \\
\hline MDate & 19383 & 3.0873 & 1.1641 & 152 & 2.65 & 0.0088 \\
\hline MDate & 19384 & 3.9333 & 0.9531 & 146 & 4.13 & $<.0001$ \\
\hline MDate & 19385 & 3.2333 & 1.3571 & 163 & 2.38 & 0.0184 \\
\hline MDate & 19386 & 1.9769 & 1.1641 & 152 & 1.70 & 0.0915 \\
\hline MDate & 19387 & 2.6569 & 1.3571 & 163 & 1.96 & 0.0520 \\
\hline MDate & 19388 & 2.0015 & 1.7170 & 169 & 1.17 & 0.2454 \\
\hline MDate & 19389 & 2.8491 & 1.3571 & 163 & 2.10 & 0.0373 \\
\hline MDate & 19390 & 1.2484 & 0.8874 & 145 & 1.41 & 0.1616 \\
\hline MDate & 19391 & 2.6256 & 1.7170 & 169 & 1.53 & 0.1281 \\
\hline MDate & 19392 & 1.8381 & 1.1641 & 152 & 1.58 & 0.1164 \\
\hline MDate & 19393 & 0.3513 & 1.3571 & 163 & 0.26 & 0.7961 \\
\hline MDate & 19394 & 0.3513 & 1.3571 & 163 & 0.26 & 0.7961 \\
\hline MDate & 19395 & 1.0654 & 1.7170 & 169 & 0.62 & 0.5358 \\
\hline
\end{tabular}

The SAS System

10:41 Thursday, March 14, 201362

Analyte $=$ Nitrate RefValue $=2$

The Mixed Procedure

Solution for Random Effects

$\begin{array}{lrrrrrr}\text { Effect } & \text { MDate } & \text { Estimate } & \begin{array}{r}\text { Std Err } \\ \text { Pred }\end{array} & \text { DF } & \text { t Value } & \operatorname{Pr}>|t| \\ \text { MDate } & 19396 & 2.0805 & 1.3571 & 163 & 1.53 & 0.1272 \\ \text { MDate } & 19397 & 1.6895 & 1.7170 & 169 & 0.98 & 0.3265 \\ \text { MDate } & 19398 & 2.5321 & 1.1641 & 152 & 2.18 & 0.0312 \\ \text { MDate } & 19399 & 2.3136 & 1.7170 & 169 & 1.35 & 0.1796\end{array}$

The SAS System

10:41 Thursday, March 14, 201363 
SRNL-STI-2013-00139

Revision 0

The Mixed Procedure

Model Information

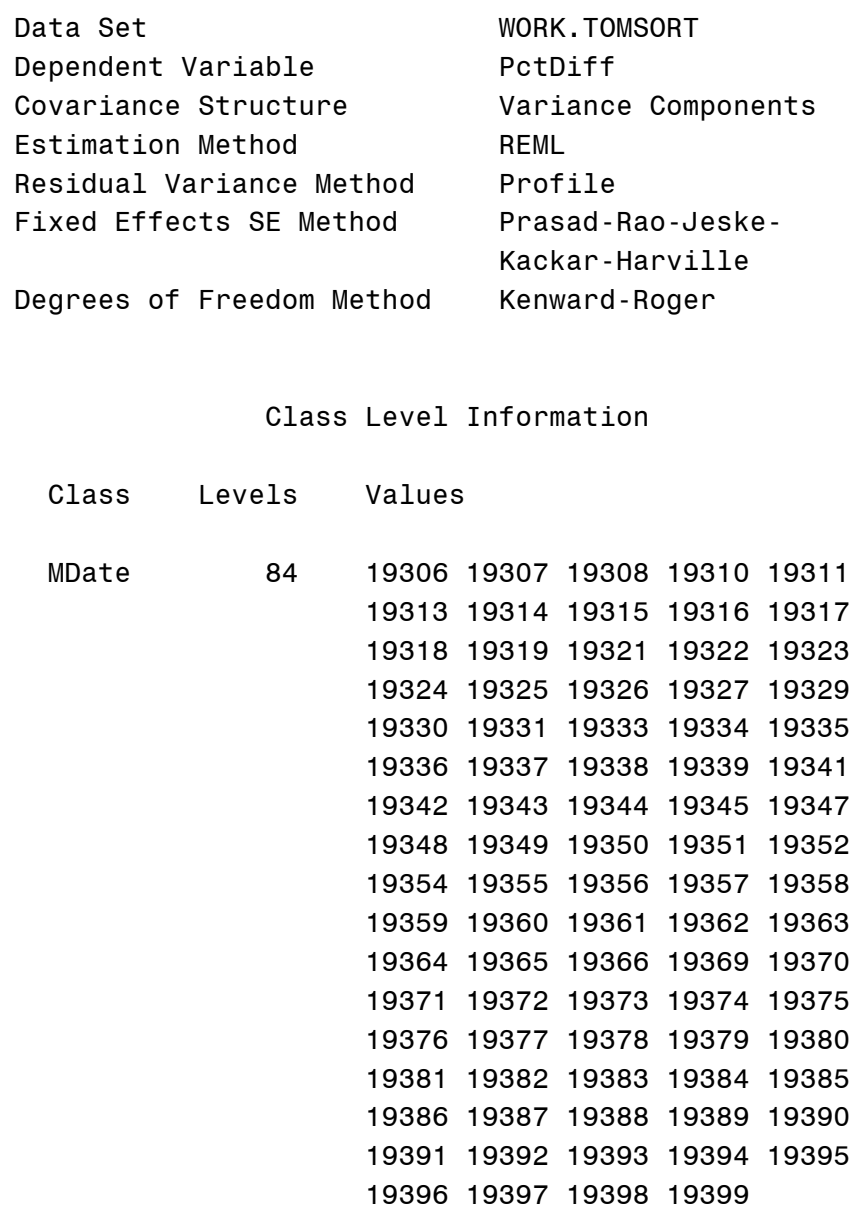

Dimensions

$\begin{array}{lr}\text { Covariance Parameters } & 2 \\ \text { Columns in X } & 1 \\ \text { Columns in Z } & 84 \\ \text { Subjects } & 1 \\ \text { Max Obs Per Subject } & 172 \\ \text { Observations Used } & 172 \\ \text { Observations Not Used } & 0 \\ \text { Total Observations } & 172\end{array}$

The SAS System 10:41 Thursday, March 14, 201364

The Mixed Procedure

Iteration History 
SRNL-STI-2013-00139

Revision 0

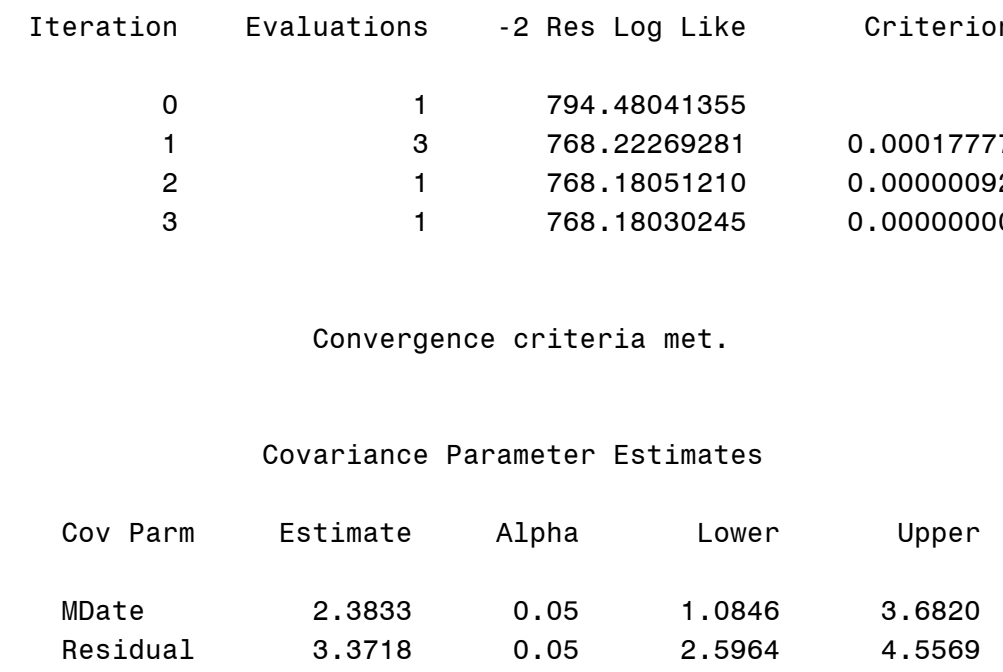

Asymptotic Covariance Matrix of Estimates

$\begin{array}{rlrr}\text { Row } & \text { Cov Parm } & \text { CovP1 } & \text { CovP2 } \\ & & & \\ 1 & \text { MDate } & 0.4391 & -0.09791 \\ 2 & \text { Residual } & -0.09791 & 0.2318\end{array}$

Fit Statistics

$\begin{array}{ll}\text {-2 Res Log Likelihood } & 768.2 \\ \text { AIC (smaller is better) } & 772.2 \\ \text { AICC (smaller is better) } & 772.3 \\ \text { BIC (smaller is better) } & 777.0\end{array}$

Null Model Likelihood Ratio Test

\begin{tabular}{|c|c|c|}
\hline DF & Chi-Square & $\mathrm{Pr}>\mathrm{ChiSq}$ \\
\hline 1 & 26.30 & $<.0001$ \\
\hline
\end{tabular}

The SAS System

10:41 Thursday, March 14, 201365

Analyte $=$ Nitrate RefValue $=16$

The Mixed Procedure

Solution for Fixed Effects

$\begin{array}{llllll}\text { Effect } & \text { Estimate } & \text { Error } & \text { DF } & t \text { Value } & \operatorname{Pr}>|t| \\ \text { Intercept } & -0.02393 & 0.2275 & 84.7 & -0.11 & 0.9165\end{array}$


SRNL-STI-2013-00139

Revision 0

Solution for Random Effects

The SAS System

10:41 Thursday, March 14, 201366

Analyte $=$ Nitrate RefValue $=16$

The Mixed Procedure

Solution for Random Effects

$\begin{array}{lrrrrrr}\text { Effect } & \text { MDate } & \text { Estimate } & \begin{array}{r}\text { Std Err } \\ \text { Pred }\end{array} & \text { DF } & \text { t Value } & \text { Pr > }|t| \\ & & & & & & \\ \text { MDate } & 19349 & 0.6052 & 1.2193 & 81 & 0.50 & 0.6210 \\ \text { MDate } & 19350 & 0.3986 & 0.8249 & 169 & 0.48 & 0.6296 \\ \text { MDate } & 19351 & 0.7827 & 1.0310 & 133 & 0.76 & 0.4491 \\ \text { MDate } & 19352 & -0.2422 & 1.0310 & 133 & -0.23 & 0.8146\end{array}$


SRNL-STI-2013-00139

Revision 0

\begin{tabular}{|c|c|c|c|c|c|c|}
\hline MDate & 19354 & 1.2904 & 0.9100 & 160 & 1.42 & 0.1581 \\
\hline MDate & 19355 & 0.5542 & 0.9100 & 160 & 0.61 & 0.5433 \\
\hline MDate & 19356 & 0.6109 & 0.9100 & 160 & 0.67 & 0.5030 \\
\hline MDate & 19357 & 0.7605 & 1.2193 & 81 & 0.62 & 0.5346 \\
\hline MDate & 19358 & 0.9658 & 1.0310 & 133 & 0.94 & 0.3506 \\
\hline MDate & 19359 & 0.7644 & 1.0310 & 133 & 0.74 & 0.4597 \\
\hline MDate & 19360 & 0.2687 & 1.2193 & 81 & 0.22 & 0.8261 \\
\hline MDate & 19361 & 0.6546 & 1.0310 & 133 & 0.63 & 0.5266 \\
\hline MDate & 19362 & -0.3524 & 1.2193 & 81 & -0.29 & 0.7733 \\
\hline MDate & 19363 & 0.01402 & 1.0310 & 133 & 0.01 & 0.9892 \\
\hline MDate & 19364 & -0.8183 & 1.2193 & 81 & -0.67 & 0.5040 \\
\hline MDate & 19365 & -0.8960 & 1.2193 & 81 & -0.73 & 0.4646 \\
\hline MDate & 19366 & -0.5077 & 1.2193 & 81 & -0.42 & 0.6782 \\
\hline MDate & 19369 & -1.4685 & 1.0310 & 133 & -1.42 & 0.1567 \\
\hline MDate & 19370 & -3.7198 & 1.0310 & 133 & -3.61 & 0.0004 \\
\hline MDate & 19371 & -3.2965 & 0.9100 & 160 & -3.62 & 0.0004 \\
\hline MDate & 19372 & -4.2781 & 0.7612 & 171 & -5.62 & $<.0001$ \\
\hline MDate & 19373 & -1.7501 & 1.2193 & 81 & -1.44 & 0.1550 \\
\hline MDate & 19374 & -0.2489 & 1.2193 & 81 & -0.20 & 0.8388 \\
\hline MDate & 19375 & -0.2489 & 1.2193 & 81 & -0.20 & 0.8388 \\
\hline MDate & 19376 & -1.5305 & 0.7612 & 171 & -2.01 & 0.0459 \\
\hline MDate & 19377 & 0.7666 & 0.9100 & 160 & 0.84 & 0.4008 \\
\hline MDate & 19378 & -0.1454 & 1.2193 & 81 & -0.12 & 0.9054 \\
\hline MDate & 19379 & -0.5075 & 0.9100 & 160 & -0.56 & 0.5778 \\
\hline MDate & 19380 & 0.03042 & 0.9100 & 160 & 0.03 & 0.9734 \\
\hline MDate & 19381 & -0.1208 & 0.8249 & 169 & -0.15 & 0.8837 \\
\hline MDate & 19382 & 1.2220 & 1.0310 & 133 & 1.19 & 0.2380 \\
\hline MDate & 19383 & 1.1488 & 0.9100 & 160 & 1.26 & 0.2086 \\
\hline MDate & 19384 & 1.3048 & 0.7612 & 171 & 1.71 & 0.0883 \\
\hline MDate & 19385 & 1.2586 & 1.0310 & 133 & 1.22 & 0.2243 \\
\hline MDate & 19386 & 0.2286 & 0.9100 & 160 & 0.25 & 0.8020 \\
\hline MDate & 19387 & 0.8743 & 1.0310 & 133 & 0.85 & 0.3980 \\
\hline MDate & 19388 & 0.8123 & 1.2193 & 81 & 0.67 & 0.5072 \\
\hline MDate & 19389 & 0.01402 & 1.0310 & 133 & 0.01 & 0.9892 \\
\hline MDate & 19390 & 0.4745 & 0.7115 & 170 & 0.67 & 0.5057 \\
\hline MDate & 19391 & 0.7087 & 1.2193 & 81 & 0.58 & 0.5627 \\
\hline MDate & 19392 & 1.2904 & 0.9100 & 160 & 1.42 & 0.1581 \\
\hline MDate & 19393 & -0.1141 & 1.0310 & 133 & -0.11 & 0.9120 \\
\hline MDate & 19394 & -1.2123 & 1.0310 & 133 & -1.18 & 0.2418 \\
\hline MDate & 19395 & -0.1195 & 1.2193 & 81 & -0.10 & 0.9222 \\
\hline
\end{tabular}

The SAS System

10:41 Thursday, March 14, 201367

Analyte $=$ Nitrate RefValue $=16$

The Mixed Procedure

Solution for Random Effects

\begin{tabular}{|c|c|c|c|c|c|c|}
\hline \multicolumn{7}{|c|}{ SLO EIT } \\
\hline Effect & MDate & Estimate & Pred & DF & t Value & $\mathrm{Pr}>|\mathrm{t}|$ \\
\hline MDate & 19396 & 0.1787 & 1.0310 & 133 & 0.17 & 0.8626 \\
\hline MDate & 19397 & 0.5017 & 1.2193 & 81 & 0.41 & 0.6818 \\
\hline MDate & 19398 & 0.5967 & 0.9100 & 160 & 0.66 & 0.5129 \\
\hline MDate & 19399 & 0.6570 & 1.2193 & 81 & 0.54 & 0.5915 \\
\hline
\end{tabular}


SRNL-STI-2013-00139

Revision 0

The SAS System

10:41 Thursday, March 14, 201368

Analyte $=$ Sol0 $x$ RefValue $=2$

The Mixed Procedure

Model Information

Data Set

WORK. TOMSORT

Dependent Variable

PctDiff

Covariance Structure

Variance Components

Estimation Method

REML

Residual Variance Method

Profile

Fixed Effects SE Method

Prasad-Rao-Jeske-

Kackar-Harville

Degrees of Freedom Method

Kenward-Roger

Class Level Information

\begin{tabular}{|c|c|c|c|c|c|}
\hline Class & Levels & Values & & & \\
\hline MDate & 82 & 1930619307 & 19308 & 19310 & 19311 \\
\hline & & 1931319314 & 19315 & 19316 & 19317 \\
\hline & & 1931819319 & 19321 & 19322 & 19323 \\
\hline & & 1932419325 & 19326 & 19327 & 19329 \\
\hline & & 1933019331 & 19333 & 19334 & 19335 \\
\hline & & 1933619337 & 19338 & 19339 & 19341 \\
\hline & & 1934219343 & 19344 & 19345 & 19347 \\
\hline & & 1934819349 & 19350 & 19351 & 19352 \\
\hline & & 1935419355 & 19356 & 19357 & 19358 \\
\hline & & 1935919360 & 19361 & 19362 & 19363 \\
\hline & & 1936419365 & 19366 & 19369 & 19370 \\
\hline & & 1937119372 & 19373 & 19375 & 19376 \\
\hline & & 1937719378 & 19379 & 19380 & 19381 \\
\hline & & 1938219383 & 19384 & 19385 & 19386 \\
\hline & & 1938719388 & 19389 & 19390 & 19392 \\
\hline & & 1939319394 & 19395 & 19396 & 19397 \\
\hline & & 1939819399 & & & \\
\hline
\end{tabular}

Dimensions

Covariance Parameters

Columns in $X$

2

Columns in $Z$

1

Subjects

82

Max Obs Per Subject

1

Observations Used

Observations Not Used

147

147

Total Observations

147

The SAS System

10:41 Thursday, March 14, 2013

69

Analyte $=$ Sol0x RefValue $=2$

The Mixed Procedure 
SRNL-STI-2013-00139

Revision 0

Iteration History

\begin{tabular}{|c|c|c|c|c|}
\hline \multicolumn{5}{|c|}{ Iteration History } \\
\hline Iteration & Evaluations & -2 Res & Log Like & Criterion \\
\hline 0 & 1 & 799 . & .79537440 & \\
\hline 1 & 2 & 760 & 21718347 & 0.00000031 \\
\hline 2 & 1 & 760 & 21710755 & 0.00000000 \\
\hline \multicolumn{5}{|c|}{ Convergence criteria met. } \\
\hline \multicolumn{5}{|c|}{ Covariance Parameter Estimates } \\
\hline Cov Parm & Estimate & Alpha & Lower & Upper \\
\hline MDate & 9.0597 & 0.05 & 5.1590 & 12.9605 \\
\hline Residual & 4.6825 & 0.05 & 3.4121 & 6.8266 \\
\hline
\end{tabular}

Asymptotic Covariance Matrix of Estimates

$\begin{array}{rlrr}\text { Row } & \text { Cov Parm } & \text { CovP1 } & \text { CovP2 } \\ & & & \\ 1 & \text { MDate } & 3.9609 & -0.4510 \\ 2 & \text { Residual } & -0.4510 & 0.6760\end{array}$

\section{Fit Statistics}

$\begin{array}{ll}\text {-2 Res Log Likelihood } & 760.2 \\ \text { AIC (smaller is better) } & 764.2 \\ \text { AICC (smaller is better) } & 764.3 \\ \text { BIC (smaller is better) } & 769.0\end{array}$

Null Model Likelihood Ratio Test

DF Chi-Square $\quad \mathrm{Pr}>\mathrm{ChiSq}$

$1 \quad 39.58<.0001$

The SAS System 10:41 Thursday, March 14, 201370

Analyte=Sol0x RefValue=2 $\ldots \ldots \ldots \ldots$

The Mixed Procedure

Solution for Fixed Effects

$\begin{array}{lrrrrr}\text { Effect } & \text { Estimate } & \begin{array}{r}\text { Standard } \\ \text { Error }\end{array} & \text { DF } & t \text { Value } & \text { Pr }>|t| \\ \text { Intercept } & 3.5352 & 0.3854 & 78.8 & 9.17 & <.0001\end{array}$


SRNL-STI-2013-00139

Revision 0

Solution for Random Effects

\begin{tabular}{|c|c|c|c|c|c|c|}
\hline & & & Std Err & & & \\
\hline Effect & MDate & Estimate & Pred & DF & t Value & $\operatorname{Pr}>|t|$ \\
\hline MDate & 19306 & 2.2842 & 1.8134 & 143 & 1.26 & 0.2098 \\
\hline MDate & 19307 & 1.2953 & 1.8134 & 143 & 0.71 & 0.4762 \\
\hline MDate & 19308 & 0.6361 & 1.8134 & 143 & 0.35 & 0.7263 \\
\hline MDate & 19310 & -0.8226 & 1.4194 & 123 & -0.58 & 0.5633 \\
\hline MDate & 19311 & -0.6824 & 1.8134 & 143 & -0.38 & 0.7072 \\
\hline MDate & 19313 & -0.4253 & 1.4194 & 123 & -0.30 & 0.7650 \\
\hline MDate & 19314 & -2.6602 & 1.8134 & 143 & -1.47 & 0.1446 \\
\hline MDate & 19315 & -1.3417 & 1.8134 & 143 & -0.74 & 0.4606 \\
\hline MDate & 19316 & -1.0121 & 1.8134 & 143 & -0.56 & 0.5776 \\
\hline MDate & 19317 & -1.3417 & 1.8134 & 143 & -0.74 & 0.4606 \\
\hline MDate & 19318 & -1.8782 & 1.2138 & 113 & -1.55 & 0.1245 \\
\hline MDate & 19319 & -2.6602 & 1.8134 & 143 & -1.47 & 0.1446 \\
\hline MDate & 19321 & -4.6804 & 1.0838 & 110 & -4.32 & $<.0001$ \\
\hline MDate & 19322 & -3.2065 & 1.4194 & 123 & -2.26 & 0.0256 \\
\hline MDate & 19323 & 4.9386 & 1.4194 & 123 & 3.48 & 0.0007 \\
\hline MDate & 19324 & 4.9213 & 1.8134 & 143 & 2.71 & 0.0075 \\
\hline MDate & 19325 & 4.1439 & 1.4194 & 123 & 2.92 & 0.0042 \\
\hline MDate & 19326 & 4.5916 & 1.8134 & 143 & 2.53 & 0.0124 \\
\hline MDate & 19327 & 1.2953 & 1.8134 & 143 & 0.71 & 0.4762 \\
\hline MDate & 19329 & 1.1640 & 1.4194 & 123 & 0.82 & 0.4138 \\
\hline MDate & 19330 & 1.1640 & 1.4194 & 123 & 0.82 & 0.4138 \\
\hline MDate & 19331 & -3.6038 & 1.4194 & 123 & -2.54 & 0.0124 \\
\hline MDate & 19333 & -0.2266 & 1.4194 & 123 & -0.16 & 0.8734 \\
\hline MDate & 19334 & -4.3084 & 1.8134 & 143 & -2.38 & 0.0188 \\
\hline MDate & 19335 & -0.6824 & 1.8134 & 143 & -0.38 & 0.7072 \\
\hline MDate & 19336 & -0.6824 & 1.8134 & 143 & -0.38 & 0.7072 \\
\hline MDate & 19337 & -0.02318 & 1.8134 & 143 & -0.01 & 0.9898 \\
\hline MDate & 19338 & -2.9898 & 1.8134 & 143 & -1.65 & 0.1014 \\
\hline MDate & 19339 & -5.1931 & 1.4194 & 123 & -3.66 & 0.0004 \\
\hline MDate & 19341 & -0.02794 & 1.4194 & 123 & -0.02 & 0.9843 \\
\hline MDate & 19342 & -1.4517 & 1.2138 & 113 & -1.20 & 0.2342 \\
\hline MDate & 19343 & -0.3528 & 1.8134 & 143 & -0.19 & 0.8460 \\
\hline MDate & 19344 & -0.3528 & 1.8134 & 143 & -0.19 & 0.8460 \\
\hline MDate & 19345 & -1.0121 & 1.8134 & 143 & -0.56 & 0.5776 \\
\hline MDate & 19347 & 0.3694 & 1.4194 & 123 & 0.26 & 0.7951 \\
\hline MDate & 19348 & -0.2266 & 1.4194 & 123 & -0.16 & 0.8734 \\
\hline
\end{tabular}

The SAS System

10:41 Thursday, March 14, 201371

Analyte=Sol0x RefValue $=2$

The Mixed Procedure

Solution for Random Effects

$\begin{array}{lrrrrrr}\text { Effect } & \text { MDate } & \text { Estimate } & \begin{array}{r}\text { Std Err } \\ \text { Pred }\end{array} & \text { DF } & \text { t Value } & \text { Pr }>|t| \\ \text { MDate } & 19349 & 0.9657 & 1.8134 & 143 & 0.53 & 0.5952 \\ \text { MDate } & 19350 & 0.1903 & 1.0838 & 110 & 0.18 & 0.8610\end{array}$


SRNL-STI-2013-00139

Revision 0

\begin{tabular}{|c|c|c|c|c|c|c|}
\hline MDate & 19351 & -1.0212 & 1.4194 & 123 & -0.72 & 0.4732 \\
\hline MDate & 19352 & -0.8226 & 1.4194 & 123 & -0.58 & 0.5633 \\
\hline MDate & 19354 & -0.3528 & 1.8134 & 143 & -0.19 & 0.8460 \\
\hline MDate & 19355 & 0.7667 & 1.4194 & 123 & 0.54 & 0.5901 \\
\hline MDate & 19356 & -0.4565 & 1.2138 & 113 & -0.38 & 0.7075 \\
\hline MDate & 19357 & 0.3065 & 1.8134 & 143 & 0.17 & 0.8660 \\
\hline MDate & 19358 & -0.8226 & 1.4194 & 123 & -0.58 & 0.5633 \\
\hline MDate & 19359 & 0.1707 & 1.4194 & 123 & 0.12 & 0.9045 \\
\hline MDate & 19360 & -0.02318 & 1.8134 & 143 & -0.01 & 0.9898 \\
\hline MDate & 19361 & -0.8226 & 1.4194 & 123 & -0.58 & 0.5633 \\
\hline MDate & 19362 & -1.6713 & 1.8134 & 143 & -0.92 & 0.3583 \\
\hline MDate & 19363 & -0.8226 & 1.4194 & 123 & -0.58 & 0.5633 \\
\hline MDate & 19364 & -2.3306 & 1.8134 & 143 & -1.29 & 0.2008 \\
\hline MDate & 19365 & -7.9343 & 1.8134 & 143 & -4.38 & $<.0001$ \\
\hline MDate & 19366 & -3.3195 & 1.8134 & 143 & -1.83 & 0.0692 \\
\hline MDate & 19369 & -4.9945 & 1.4194 & 123 & -3.52 & 0.0006 \\
\hline MDate & 19370 & -7.5771 & 1.4194 & 123 & -5.34 & $<.0001$ \\
\hline MDate & 19371 & -2.0204 & 1.2138 & 113 & -1.66 & 0.0988 \\
\hline MDate & 19372 & -0.8830 & 1.2138 & 113 & -0.73 & 0.4684 \\
\hline MDate & 19373 & 2.6139 & 1.8134 & 143 & 1.44 & 0.1516 \\
\hline MDate & 19375 & 2.6139 & 1.8134 & 143 & 1.44 & 0.1516 \\
\hline MDate & 19376 & 2.6713 & 1.2138 & 113 & 2.20 & 0.0298 \\
\hline MDate & 19377 & 3.3493 & 1.4194 & 123 & 2.36 & 0.0199 \\
\hline MDate & 19378 & 2.9435 & 1.8134 & 143 & 1.62 & 0.1067 \\
\hline MDate & 19379 & 1.6250 & 1.8134 & 143 & 0.90 & 0.3717 \\
\hline MDate & 19380 & 1.7600 & 1.4194 & 123 & 1.24 & 0.2174 \\
\hline MDate & 19381 & 0.6330 & 1.0838 & 110 & 0.58 & 0.5604 \\
\hline MDate & 19382 & -0.2266 & 1.4194 & 123 & -0.16 & 0.8734 \\
\hline MDate & 19383 & 1.5339 & 1.2138 & 113 & 1.26 & 0.2089 \\
\hline MDate & 19384 & 4.6810 & 0.9928 & 110 & 4.71 & $<.0001$ \\
\hline MDate & 19385 & 4.1439 & 1.4194 & 123 & 2.92 & 0.0042 \\
\hline MDate & 19386 & 2.5291 & 1.2138 & 113 & 2.08 & 0.0394 \\
\hline MDate & 19387 & 2.6139 & 1.8134 & 143 & 1.44 & 0.1516 \\
\hline MDate & 19388 & 1.9546 & 1.8134 & 143 & 1.08 & 0.2829 \\
\hline MDate & 19389 & 2.9556 & 1.2138 & 113 & 2.44 & 0.0164 \\
\hline MDate & 19390 & -1.4702 & 1.0838 & 110 & -1.36 & 0.1777 \\
\hline MDate & 19392 & 0.6361 & 1.8134 & 143 & 0.35 & 0.7263 \\
\hline MDate & 19393 & -0.2266 & 1.4194 & 123 & -0.16 & 0.8734 \\
\hline MDate & 19394 & -0.4253 & 1.4194 & 123 & -0.30 & 0.7650 \\
\hline MDate & 19395 & 1.6250 & 1.8134 & 143 & 0.90 & 0.3717 \\
\hline MDate & 19396 & 2.5547 & 1.4194 & 123 & 1.80 & 0.0744 \\
\hline MDate & 19397 & 1.9546 & 1.8134 & 143 & 1.08 & 0.2829 \\
\hline
\end{tabular}

The SAS System

10:41 Thursday, March 14, 201372

Analyte $=$ Solox RefValue $=2$

The Mixed Procedure

Solution for Random Effects

$\begin{array}{lrrrrrr}\text { Effect } & \text { MDate } & \text { Estimate } & \begin{array}{r}\text { Std Err } \\ \text { Pred }\end{array} & \text { DF } & \text { t Value } & \text { Pr }>|t| \\ \text { MDate } & 19398 & 2.5291 & 1.2138 & 113 & 2.08 & 0.0394 \\ \text { MDate } & 19399 & 2.9435 & 1.8134 & 143 & 1.62 & 0.1067\end{array}$


SRNL-STI-2013-00139

Revision 0

The SAS System

10:41 Thursday, March 14, 201373

Analyte=Sol0x RefValue $=16$

The Mixed Procedure

Model Information

Data Set

WORK. TOMSORT

Dependent Variable

PctDiff

Covariance Structure

Variance Components

Estimation Method

REML

Residual Variance Method

Profile

Fixed Effects SE Method

Prasad-Rao-Jeske-

Kackar-Harville

Degrees of Freedom Method

Kenward-Roger

Class Level Information

\begin{tabular}{|c|c|c|c|c|c|}
\hline Class & Levels & Values & & & \\
\hline MDate & 82 & 1930619307 & 19308 & 19310 & 19311 \\
\hline & & 1931319314 & 19315 & 19316 & 19317 \\
\hline & & 1931819319 & 19321 & 19322 & 19323 \\
\hline & & 1932419325 & 19326 & 19327 & 19329 \\
\hline & & 1933019331 & 19333 & 19334 & 19335 \\
\hline & & 1933619337 & 19338 & 19339 & 19341 \\
\hline & & 1934219343 & 19344 & 19345 & 19347 \\
\hline & & 1934819349 & 19350 & 19351 & 19352 \\
\hline & & 1935419355 & 19356 & 19357 & 19358 \\
\hline & & 1935919360 & 19361 & 19362 & 19363 \\
\hline & & 1936419365 & 19366 & 19369 & 19370 \\
\hline & & 1937119372 & 19373 & 19375 & 19376 \\
\hline & & 1937719378 & 19379 & 19380 & 19381 \\
\hline & & 1938219383 & 19384 & 19385 & 19386 \\
\hline & & 1938719388 & 19389 & 19390 & 19392 \\
\hline & & 1939319394 & 19395 & 19396 & 19397 \\
\hline & & 1939819399 & & & \\
\hline
\end{tabular}

Dimensions

Covariance Parameters

Columns in $X$

2

Columns in $Z$

1

Subjects

82

Max Obs Per Subject

1

Observations Used

Observations Not Used

147

147

Total Observations

147

The SAS System

10:41 Thursday, March 14, 2013

Analyte=Sol0x RefValue $=16$

The Mixed Procedure 
SRNL-STI-2013-00139

Revision 0

Iteration History

\begin{tabular}{|c|c|c|c|c|}
\hline \multicolumn{5}{|c|}{ Iteration History } \\
\hline Iteration & Evaluations & -2 Res & Log Like & Criterion \\
\hline 0 & 1 & 731 & .58740679 & \\
\hline 1 & 3 & 697 & .43670832 & 0.00012685 \\
\hline 2 & 1 & 697 & .40849212 & 0.00000042 \\
\hline 3 & 1 & 697 & .40840142 & 0.000000 \\
\hline \multicolumn{5}{|c|}{ Convergence criteria met. } \\
\hline \multicolumn{5}{|c|}{ Covariance Parameter Estimates } \\
\hline Cov Parm & Estimate & Alpha & Lower & Upper \\
\hline MDate & 4.5755 & 0.05 & 2.4668 & 6.6842 \\
\hline Residual & 3.5928 & 0.05 & 2.6604 & 5.1208 \\
\hline
\end{tabular}

Asymptotic Covariance Matrix of Estimates

$\begin{array}{rlrr}\text { Row } & \text { Cov Parm } & \text { CovP1 } & \text { CovP2 } \\ & & & \\ 1 & \text { MDate } & 1.1575 & -0.1691 \\ 2 & \text { Residual } & -0.1691 & 0.3554\end{array}$

Fit Statistics

$\begin{array}{ll}\text {-2 Res Log Likelihood } & 697.4 \\ \text { AIC (smaller is better) } & 701.4 \\ \text { AICC (smaller is better) } & 701.5 \\ \text { BIC (smaller is better) } & 706.2\end{array}$

Null Model Likelihood Ratio Test

DF Chi-Square $\quad \mathrm{Pr}>$ Chisq

$1 \quad 34.18<.0001$

The SAS System 10:41 Thursday, March 14, 201375

Analyte $=$ Solox RefValue $=16$

The Mixed Procedure

Solution for Fixed Effects

Effect Estimate $\begin{array}{r}\text { Standard } \\ \text { Error }\end{array} \quad \mathrm{DF}$ Value $>|t|$ 
SRNL-STI-2013-00139

Revision 0

$\begin{array}{llllll}\text { Intercept } & -0.6933 & 0.2912 & 84.7 & -2.38 & 0.0195\end{array}$

Solution for Random Effects

\begin{tabular}{|c|c|c|c|c|c|c|}
\hline & & & Std Err & & & \\
\hline Effect & MDate & Estimate & Pred & DF & t Value & $\operatorname{Pr}>|t|$ \\
\hline MDate & 19306 & 1.3336 & 1.4629 & 138 & 0.91 & 0.3635 \\
\hline MDate & 19307 & 0.3534 & 1.4629 & 138 & 0.24 & 0.809 \\
\hline MDate & 19308 & 0.003276 & 1.4629 & 138 & 0.00 & 0.998 \\
\hline MDate & 19310 & -0.8710 & 1.1778 & 143 & -0.74 & 0.460 \\
\hline MDate & 19311 & -0.9070 & 1.4629 & 138 & -0.62 & 0.536 \\
\hline MDate & 19313 & 0.2735 & 1.1778 & 143 & 0.23 & 0.816 \\
\hline MDate & 19314 & 0.3184 & 1.4629 & 138 & 0.22 & 0.828 \\
\hline MDate & 19315 & -0.2768 & 1.4629 & 138 & -0.19 & 0.850 \\
\hline MDate & 19316 & 0.003276 & 1.4629 & 138 & 0.00 & 0.998 \\
\hline MDate & 19317 & -0.2418 & 1.4629 & 138 & -0.17 & 0.869 \\
\hline MDate & 19318 & -1.3658 & 1.0172 & 133 & -1.34 & 0.181 \\
\hline MDate & 19319 & -2.0623 & 1.4629 & 138 & -1.41 & 0.1609 \\
\hline MDate & 19321 & -0.2302 & 0.9120 & 127 & -0.25 & 0.801 \\
\hline MDate & 19322 & -0.8720 & 1.4629 & 138 & -0.60 & 0.5521 \\
\hline MDate & 19323 & -1.0954 & 1.1778 & 143 & -0.93 & 0.353 \\
\hline MDate & 19324 & 0.9485 & 1.4629 & 138 & 0.65 & 0.517 \\
\hline MDate & 19325 & 1.1935 & 1.0172 & 133 & 1.17 & 0.242 \\
\hline MDate & 19326 & 1.0886 & 1.4629 & 138 & 0.74 & 0.458 \\
\hline MDate & 19327 & -1.3971 & 1.4629 & 138 & -0.96 & 0.341 \\
\hline MDate & 19329 & -0.1304 & 1.1778 & 143 & -0.11 & 0.912 \\
\hline MDate & 19330 & -0.3548 & 1.1778 & 143 & -0.30 & 0.763 \\
\hline MDate & 19331 & 0.1613 & 1.1778 & 143 & 0.14 & 0.891 \\
\hline MDate & 19333 & 0.8345 & 1.1778 & 143 & 0.71 & 0.479 \\
\hline MDate & 19334 & -4.4430 & 1.4629 & 138 & -3.04 & 0.002 \\
\hline MDate & 19335 & -1.0470 & 1.4629 & 138 & -0.72 & 0.475 \\
\hline MDate & 19336 & 0.3184 & 1.4629 & 138 & 0.22 & 0.828 \\
\hline MDate & 19337 & 0.7735 & 1.4629 & 138 & 0.53 & 0.597 \\
\hline MDate & 19338 & -1.0120 & 1.4629 & 138 & -0.69 & 0.490 \\
\hline MDate & 19339 & -3.4740 & 1.1778 & 143 & -2.95 & 0.003 \\
\hline MDate & 19341 & 0.5203 & 1.1778 & 143 & 0.44 & 0.659 \\
\hline MDate & 19342 & -0.5072 & 1.0172 & 133 & -0.50 & 0.618 \\
\hline MDate & 19343 & -0.1368 & 1.4629 & 138 & -0.09 & 0.925 \\
\hline MDate & 19344 & -0.2418 & 1.4629 & 138 & -0.17 & 0.869 \\
\hline MDate & 19345 & -0.1018 & 1.4629 & 138 & -0.07 & 0.944 \\
\hline MDate & 19347 & -0.9607 & 1.1778 & 143 & -0.82 & 0.4160 \\
\hline MDate & 19348 & 0.4530 & 1.1778 & 143 & 0.38 & 0.7011 \\
\hline
\end{tabular}

The SAS System

10:41 Thursday, March 14, 201376

Analyte $=$ Sol0x RefValue $=16$

The Mixed Procedure

Solution for Random Effects

$\begin{array}{lrrrrrr}\text { Effect } & \text { MDate } & \text { Estimate } & \begin{array}{r}\text { Std Err } \\ \text { Pred }\end{array} & \text { DF } & t \text { Value } & \text { Pr }>|t| \\ \text { MDate } & 19349 & 0.5634 & 1.4629 & 138 & 0.39 & 0.7007\end{array}$


SRNL-STI-2013-00139

Revision 0

\begin{tabular}{|c|c|c|c|c|c|c|}
\hline MDate & 19350 & -0.02123 & 0.9120 & 127 & -0.02 & 0.9815 \\
\hline MDate & 19351 & 0.5876 & 1.1778 & 143 & 0.50 & 0.6186 \\
\hline MDate & 19352 & -0.4519 & 1.4629 & 138 & -0.31 & 0.7579 \\
\hline MDate & 19354 & 0.6685 & 1.4629 & 138 & 0.46 & 0.6484 \\
\hline MDate & 19355 & -0.04490 & 1.0172 & 133 & -0.04 & 0.9649 \\
\hline MDate & 19356 & 0.3844 & 1.0172 & 133 & 0.38 & 0.7061 \\
\hline MDate & 19357 & 0.7035 & 1.4629 & 138 & 0.48 & 0.6314 \\
\hline MDate & 19358 & 0.7223 & 1.1778 & 143 & 0.61 & 0.5407 \\
\hline MDate & 19359 & 0.3632 & 1.1778 & 143 & 0.31 & 0.7582 \\
\hline MDate & 19360 & -0.1368 & 1.4629 & 138 & -0.09 & 0.9257 \\
\hline MDate & 19361 & -0.2202 & 1.1778 & 143 & -0.19 & 0.8520 \\
\hline MDate & 19362 & -1.1170 & 1.4629 & 138 & -0.76 & 0.4464 \\
\hline MDate & 19363 & -0.8934 & 1.1778 & 143 & -0.76 & 0.4494 \\
\hline MDate & 19364 & -1.7472 & 1.4629 & 138 & -1.19 & 0.2344 \\
\hline MDate & 19365 & -1.9223 & 1.4629 & 138 & -1.31 & 0.1910 \\
\hline MDate & 19366 & -1.5021 & 1.4629 & 138 & -1.03 & 0.3063 \\
\hline MDate & 19369 & -2.8457 & 1.1778 & 143 & -2.42 & 0.0169 \\
\hline MDate & 19370 & -5.7404 & 1.1778 & 143 & -4.87 & $<.0001$ \\
\hline MDate & 19371 & -4.8168 & 1.0172 & 133 & -4.74 & $<.0001$ \\
\hline MDate & 19372 & -5.2791 & 1.0172 & 133 & -5.19 & $<.0001$ \\
\hline MDate & 19373 & -2.5874 & 1.4629 & 138 & -1.77 & 0.0792 \\
\hline MDate & 19375 & 1.0886 & 1.4629 & 138 & 0.74 & 0.4581 \\
\hline MDate & 19376 & 0.9788 & 1.0172 & 133 & 0.96 & 0.3377 \\
\hline MDate & 19377 & 2.6297 & 1.1778 & 143 & 2.23 & 0.0271 \\
\hline MDate & 19378 & 1.1586 & 1.4629 & 138 & 0.79 & 0.4297 \\
\hline MDate & 19379 & 0.7735 & 1.4629 & 138 & 0.53 & 0.5978 \\
\hline MDate & 19380 & 1.7545 & 1.1778 & 143 & 1.49 & 0.1385 \\
\hline MDate & 19381 & 1.9379 & 0.9120 & 127 & 2.13 & 0.0355 \\
\hline MDate & 19382 & 3.1009 & 1.1778 & 143 & 2.63 & 0.0094 \\
\hline MDate & 19383 & 2.9767 & 1.0172 & 133 & 2.93 & 0.0040 \\
\hline MDate & 19384 & 2.9328 & 0.8366 & 124 & 3.51 & 0.0006 \\
\hline MDate & 19385 & 2.6521 & 1.1778 & 143 & 2.25 & 0.0259 \\
\hline MDate & 19386 & 1.4577 & 1.0172 & 133 & 1.43 & 0.1542 \\
\hline MDate & 19387 & 1.6837 & 1.4629 & 138 & 1.15 & 0.2517 \\
\hline MDate & 19388 & 1.5787 & 1.4629 & 138 & 1.08 & 0.2824 \\
\hline MDate & 19389 & 1.3091 & 1.0172 & 133 & 1.29 & 0.2004 \\
\hline MDate & 19390 & 0.7624 & 0.9120 & 127 & 0.84 & 0.4047 \\
\hline MDate & 19392 & 1.8238 & 1.4629 & 138 & 1.25 & 0.2146 \\
\hline MDate & 19393 & 0.8120 & 1.1778 & 143 & 0.69 & 0.4916 \\
\hline MDate & 19394 & -0.1978 & 1.1778 & 143 & -0.17 & 0.8669 \\
\hline MDate & 19395 & 0.7035 & 1.4629 & 138 & 0.48 & 0.6314 \\
\hline MDate & 19396 & 1.3506 & 1.1778 & 143 & 1.15 & 0.2534 \\
\hline MDate & 19397 & 1.5787 & 1.4629 & 138 & 1.08 & 0.2824 \\
\hline
\end{tabular}

The SAS System

10:41 Thursday, March 14, 201377

Analyte $=$ Sol0x RefValue $=16$

The Mixed Procedure

Solution for Random Effects

$\begin{array}{lrrrrrr}\text { Effect } & \text { MDate } & \text { Estimate } & \begin{array}{r}\text { Std Err } \\ \text { Pred }\end{array} & \text { DF } & \text { t Value } & \text { Pr }>|t| \\ \text { MDate } & 19398 & 1.9200 & 1.0172 & 133 & 1.89 & 0.0613 \\ \text { MDate } & 19399 & 1.7188 & 1.4629 & 138 & 1.17 & 0.2421\end{array}$


SRNL-STI-2013-00139

Revision 0

The SAS System

10:41 Thursday, March 14, 2013

78

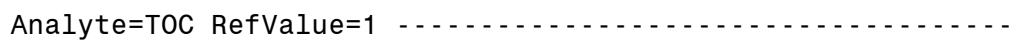

The Mixed Procedure

Model Information

Data Set

Dependent Variable

Covariance Structure

Estimation Method

Residual Variance Method

Fixed Effects SE Method

Degrees of Freedom Method
WORK. TOMSORT

PctDiff

Variance Components

REML

Profile

Prasad-Rao-Jeske-

Kackar-Harville

Kenward-Roger

Class Level Information

$\begin{array}{llllllll}\text { Class } & \text { Levels } & \text { Values } \\ \text { MDate } & 21 & 19281 & 19290 & 19318 & 19322 & 19331 \\ & & 19333 & 19336 & 19340 & 19341 & 19350 \\ & 19351 & 19352 & 19370 & 19371 & 19372 \\ & & 19380 & 19381 & 19384 & 19386 & 19390 \\ & & 19398 & & & & \end{array}$

Dimensions

Covariance Parameters

2

Columns in $X$

Columns in $Z$

Subjects

Max Obs Per Subject

Observations Used

Observations Not Used

Total Observations

1

92

92

0

92

\section{Iteration History}

$\begin{array}{rrrr}\text { Iteration } & \text { Evaluations } & -2 \text { Res Log Like } & \text { Criterion } \\ 0 & 1 & 499.35938572 & \\ 1 & 2 & 469.80798070 & 0.00006352 \\ 2 & 1 & 469.79800150 & 0.00000023 \\ 3 & 1 & 469.79796611 & 0.00000000\end{array}$

Convergence criteria met.

The SAS System

10:41 Thursday, March 14, 2013

79 
SRNL-STI-2013-00139

Revision 0

The Mixed Procedure

Covariance Parameter Estimates

$\begin{array}{lrrrr}\text { Cov Parm } & \text { Estimate } & \text { Alpha } & \text { Lower } & \text { Upper } \\ & & & & \\ \text { MDate } & 7.3745 & 0.05 & 1.7828 & 12.9663 \\ \text { Residual } & 6.6186 & 0.05 & 4.8838 & 9.4799\end{array}$

Asymptotic Covariance Matrix of Estimates

$\begin{array}{rlrr}\text { Row } & \text { Cov Parm } & \text { CovP1 } & \text { CovP2 } \\ & & & \\ 1 & \text { MDate } & 8.1397 & -0.3067 \\ 2 & \text { Residual } & -0.3067 & 1.2368\end{array}$

Fit Statistics

$\begin{array}{ll}-2 \text { Res Log Likelihood } & 469.8 \\ \text { AIC (smaller is better) } & 473.8 \\ \text { AICC (smaller is better) } & 473.9 \\ \text { BIC (smaller is better) } & 475.9\end{array}$

Null Model Likelihood Ratio Test

DF Chi-Square $\quad \operatorname{Pr}>$ ChiSq

$1 \quad 29.56 \quad<.0001$

Solution for Fixed Effects

$\begin{array}{lrrrrr}\text { Effect } & \text { Estimate } & \begin{array}{r}\text { Standard } \\ \text { Error }\end{array} & \text { DF } & t \text { Value } & \operatorname{Pr}>|t| \\ \text { Intercept } & -0.1646 & 0.6527 & 19.8 & -0.25 & 0.8034\end{array}$

Solution for Random Effects

$\begin{array}{lrrrrrr}\text { Effect } & \text { MDate } & \text { Estimate } & \begin{array}{r}\text { Std Err } \\ \text { Pred }\end{array} & \text { DF } & \text { t Value } & \operatorname{Pr}>|t| \\ \text { MDate } & 19281 & -0.6823 & 1.3072 & 71.5 & -0.52 & 0.6033 \\ \text { MDate } & 19290 & -2.1116 & 1.3072 & 71.5 & -1.62 & 0.1106 \\ \text { MDate } & 19318 & -2.9283 & 1.3072 & 71.5 & -2.24 & 0.0282 \\ \text { MDate } & 19322 & -0.06972 & 1.3072 & 71.5 & -0.05 & 0.9576 \\ \text { MDate } & 19331 & 3.8098 & 1.3072 & 71.5 & 2.91 & 0.0048\end{array}$

The SAS System 10:41 Thursday, March 14, 201380 
SRNL-STI-2013-00139

Revision 0

The Mixed Procedure

Solution for Random Effects

$\begin{array}{lrrrrrr}\text { Effect } & \text { MDate } & \text { Estimate } & \begin{array}{r}\text { Std Err } \\ \text { Pred }\end{array} & \text { DF } & \text { t Value } & \text { Pr }>|t| \\ \text { MDate } & 19333 & 1.5638 & 1.3072 & 71.5 & 1.20 & 0.2355 \\ \text { MDate } & 19336 & -0.4781 & 1.3072 & 71.5 & -0.37 & 0.7156 \\ \text { MDate } & 19340 & 0.3387 & 1.3072 & 71.5 & 0.26 & 0.7963 \\ \text { MDate } & 19341 & -0.2739 & 1.3072 & 71.5 & -0.21 & 0.8346 \\ \text { MDate } & 19350 & -1.4990 & 1.3072 & 71.5 & -1.15 & 0.2553 \\ \text { MDate } & 19351 & -1.0906 & 1.3072 & 71.5 & -0.83 & 0.4069 \\ \text { MDate } & 19352 & 1.9721 & 1.3072 & 71.5 & 1.51 & 0.1358 \\ \text { MDate } & 19370 & -2.3158 & 1.3072 & 71.5 & -1.77 & 0.0807 \\ \text { MDate } & 19371 & -2.5200 & 1.3072 & 71.5 & -1.93 & 0.0578 \\ \text { MDate } & 19372 & -2.6617 & 1.0557 & 65.4 & -2.52 & 0.0141 \\ \text { MDate } & 19380 & 4.4224 & 1.3072 & 71.5 & 3.38 & 0.0012 \\ \text { MDate } & 19381 & -2.3158 & 1.3072 & 71.5 & -1.77 & 0.0807 \\ \text { MDate } & 19384 & 0.3387 & 1.3072 & 71.5 & 0.26 & 0.7963 \\ \text { MDate } & 19386 & 0.5428 & 1.3072 & 71.5 & 0.42 & 0.6792 \\ \text { MDate } & 19390 & -0.3015 & 1.0557 & 65.4 & -0.29 & 0.7761 \\ \text { MDate } & 19398 & 6.2601 & 1.3072 & 71.5 & 4.79 & <.0001\end{array}$

The SAS System

10:41 Thursday, March 14, 201381

Analyte $=$ TOC RefValue $=20$

The Mixed Procedure

Model Information

Data Set
Dependent Variable
Covariance Structure
Estimation Method
Residual Variance Method
Fixed Effects SE Method
Degrees of Freedom Method

Data Set

Estimation Method

Degrees of Freedom Method
WORK. TOMSORT

PctDiff

Variance Components

REML

Profile

Prasad-Rao-Jeske-

Kackar-Harville

Kenward-Roger

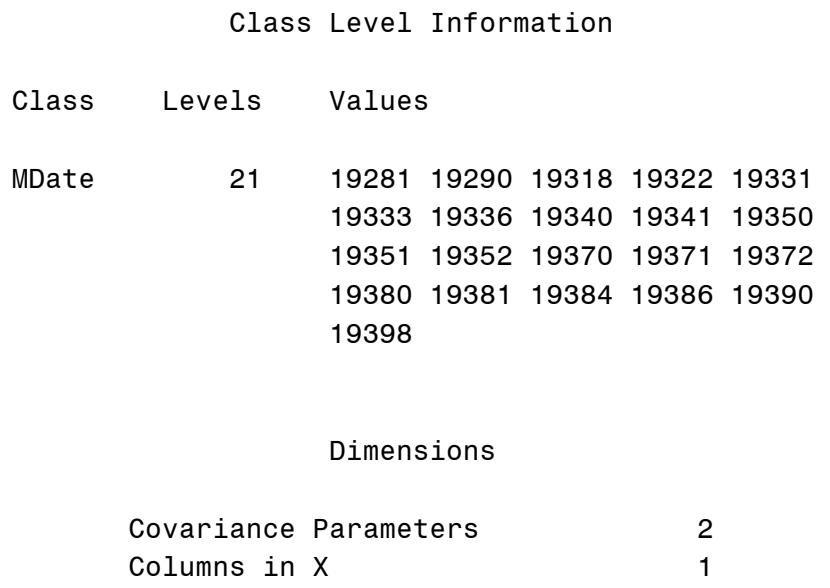


SRNL-STI-2013-00139

Revision 0

$\begin{array}{lr}\text { Columns in Z } & 21 \\ \text { Subjects } & 1 \\ \text { Max Obs Per Subject } & 92 \\ \text { Observations Used } & 92 \\ \text { Observations Not Used } & 0 \\ \text { Total Observations } & 92\end{array}$

\begin{tabular}{ccrl}
\multicolumn{5}{c}{ Iteration History } \\
Iteration & Evaluations & -2 Res Log Like & Criterion \\
& & & \\
1 & 1 & 407.75857326 & \\
2 & 2 & 311.16743392 & 0.00223033 \\
3 & 1 & 298.09650757 & 0.00098587 \\
4 & 1 & 298.04074351 & 0.00003012 \\
5 & 1 & 298.03873775 & 0.00000002 \\
& 1 & 298.03873621 & 0.00000000 \\
& & & \\
& The SAS System & $10: 41$ Thursday, March 14,201382
\end{tabular}

Analyte $=$ TOC RefValue $=20$

The Mixed Procedure

Convergence criteria met.

Covariance Parameter Estimates

$\begin{array}{lrrrr}\text { Cov Parm } & \text { Estimate } & \text { Alpha } & \text { Lower } & \text { Upper } \\ & & & & \\ \text { MDate } & 4.0095 & 0.05 & 1.4229 & 6.5962 \\ \text { Residual } & 0.7297 & 0.05 & 0.5387 & 1.0444\end{array}$

Asymptotic Covariance Matrix of Estimates

$\begin{array}{rlrr}\text { Row } & \text { Cov Parm } & \text { CovP1 } & \text { CovP2 } \\ & & & \\ 1 & \text { MDate } & 1.7417 & -0.00313 \\ 2 & \text { Residual } & -0.00313 & 0.01498\end{array}$

Fit Statistics

$\begin{array}{ll}-2 \text { Res Log Likelihood } & 298.0 \\ \text { AIC (smaller is better) } & 302.0 \\ \text { AICC (smaller is better) } & 302.2 \\ \text { BIC (smaller is better) } & 304.1\end{array}$

Null Model Likelihood Ratio Test

$$
\begin{aligned}
& \text { DF Chi-Square } \quad \mathrm{Pr}>\text { ChiSq } \\
& 1 \quad 109.72<.0001
\end{aligned}
$$


SRNL-STI-2013-00139

Revision 0

Solution for Fixed Effects

$\begin{array}{lrrrrr}\text { Effect } & \text { Estimate } & \begin{array}{r}\text { Standard } \\ \text { Error }\end{array} & \text { DF } & t \text { Value } & \operatorname{Pr}>|t| \\ \text { Intercept } & -0.2215 & 0.4463 & 20.1 & -0.50 & 0.6251\end{array}$

Solution for Random Effects

$\begin{array}{lrrrrrr}\text { Effect } & \text { MDate } & \text { Estimate } & \begin{array}{r}\text { Std Err } \\ \text { Pred }\end{array} & \text { DF } & \text { t Value } & \text { Pr }>|t| \\ \text { MDate } & 19281 & -3.9608 & 0.5989 & 52 & -6.61 & <.0001 \\ \text { MDate } & 19290 & -4.6781 & 0.5989 & 52 & -7.81 & <.0001\end{array}$

The SAS System 10:41 Thursday, March 14, 201383

Analyte $=$ TOC RefValue $=20$

The Mixed Procedure

Solution for Random Effects

$\begin{array}{lrrrrrr}\text { Effect } & \text { MDate } & \text { Estimate } & \begin{array}{r}\text { Std Err } \\ \text { Pred }\end{array} & \text { DF } & t \text { Value } & \text { Pr }>|t| \\ \text { MDate } & 19318 & -0.5533 & 0.5989 & 52 & -0.92 & 0.3598 \\ \text { MDate } & 19322 & -0.5892 & 0.5989 & 52 & -0.98 & 0.3297 \\ \text { MDate } & 19331 & 0.8455 & 0.5989 & 52 & 1.41 & 0.1639 \\ \text { MDate } & 19333 & 1.6107 & 0.5989 & 52 & 2.69 & 0.0096 \\ \text { MDate } & 19336 & 0.4988 & 0.5989 & 52 & 0.83 & 0.4087 \\ \text { MDate } & 19340 & -0.5533 & 0.5989 & 52 & -0.92 & 0.3598 \\ \text { MDate } & 19341 & -0.3381 & 0.5989 & 52 & -0.56 & 0.5748 \\ \text { MDate } & 19350 & 0.2238 & 0.5989 & 52 & 0.37 & 0.7101 \\ \text { MDate } & 19351 & -0.1827 & 0.5989 & 52 & -0.31 & 0.7616 \\ \text { MDate } & 19352 & -1.1631 & 0.5989 & 52 & -1.94 & 0.0575 \\ \text { MDate } & 19370 & 1.1803 & 0.5989 & 52 & 1.97 & 0.0541 \\ \text { MDate } & 19371 & 2.7107 & 0.5989 & 52 & 4.53 & <.0001 \\ \text { MDate } & 19372 & 3.7732 & 0.5293 & 37.1 & 7.13 & <.0001 \\ \text { MDate } & 19380 & -0.3381 & 0.5989 & 52 & -0.56 & 0.5748 \\ \text { MDate } & 19381 & 1.5868 & 0.5989 & 52 & 2.65 & 0.0106 \\ \text { MDate } & 19384 & -0.05116 & 0.5989 & 52 & -0.09 & 0.9322 \\ \text { MDate } & 19386 & -2.2391 & 0.5989 & 52 & -3.74 & 0.0005 \\ \text { MDate } & 19390 & 1.3716 & 0.5293 & 37.1 & 2.59 & 0.0136 \\ \text { MDate } & 19398 & 0.8455 & 0.5989 & 52 & 1.41 & 0.1639\end{array}$

The SAS System

10:41 Thursday, March 14, 201384

84

Analyte=Tot0x RefValue $=2$

The Mixed Procedure

Model Information 
SRNL-STI-2013-00139

Revision 0

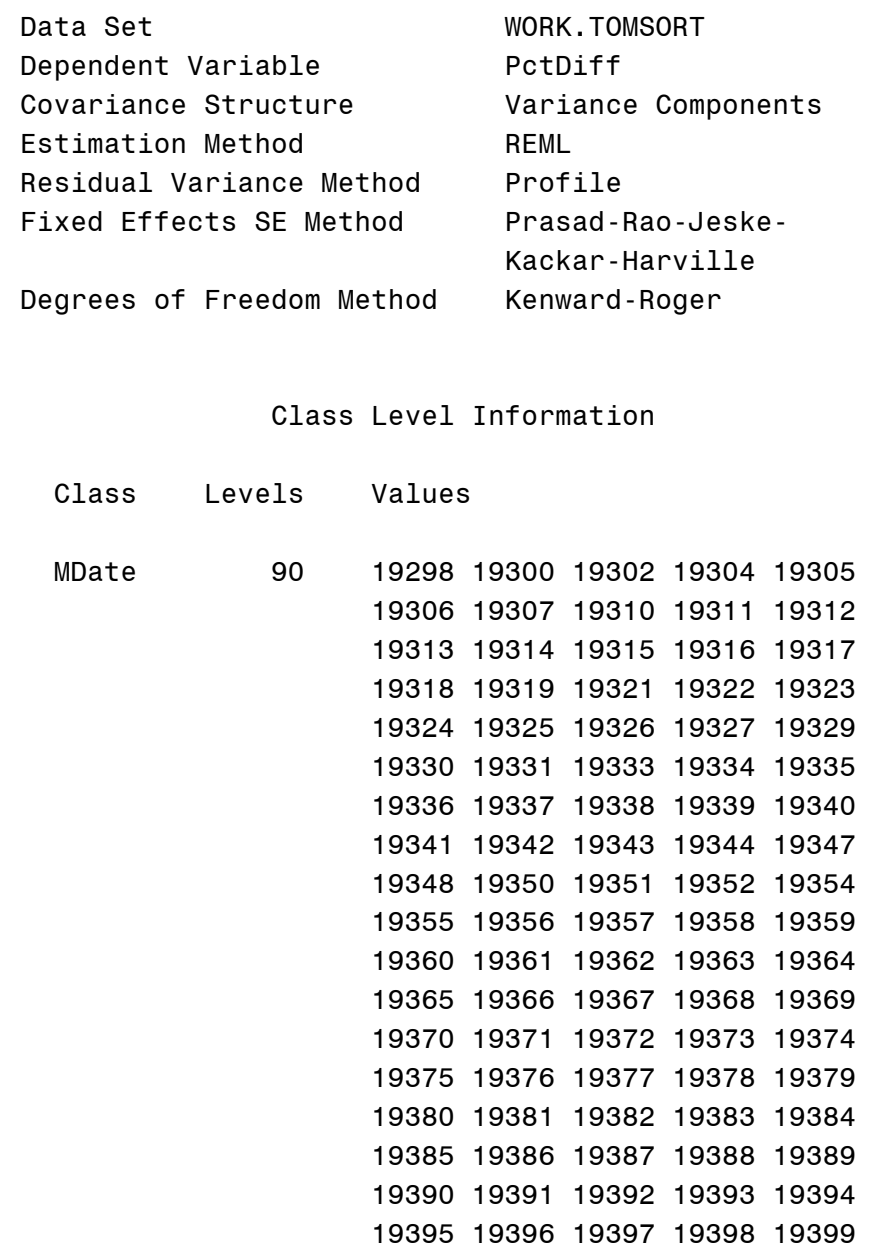

Dimensions

$\begin{array}{lr}\text { Covariance Parameters } & 2 \\ \text { Columns in X } & 1 \\ \text { Columns in Z } & 90 \\ \text { Subjects } & 1 \\ \text { Max Obs Per Subject } & 145 \\ \text { Observations Used } & 145 \\ \text { Observations Not Used } & 0 \\ \text { Total Observations } & 145\end{array}$

The SAS System 10:41 Thursday, March 14, 201385

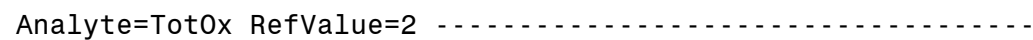

The Mixed Procedure

Iteration History

$\begin{array}{rrrr}\text { Iteration } & \text { Evaluations } & -2 \text { Res Log Like } & \text { Criterion } \\ 0 & 1 & 886.08626499 & \\ 1 & 2 & 803.45367835 & 0.00101122\end{array}$


SRNL-STI-2013-00139

Revision 0

$\begin{array}{llll}2 & 1 & 803.22140282 & 0.00003580 \\ 3 & 1 & 803.21155644 & 0.00000004 \\ 4 & 1 & 803.21154614 & 0.00000000\end{array}$

Convergence criteria met.

Covariance Parameter Estimates

$\begin{array}{lrrrr}\text { Cov Parm } & \text { Estimate } & \text { Alpha } & \text { Lower } & \text { Upper } \\ & & & & \\ \text { MDate } & 21.1429 & 0.05 & 14.1319 & 28.1539 \\ \text { Residual } & 3.8532 & 0.05 & 2.7565 & 5.7680\end{array}$

Asymptotic Covariance Matrix of Estimates

$\begin{array}{rlrr}\text { Row } & \text { Cov Parm } & \text { CovP1 } & \text { CovP2 } \\ & & & \\ 1 & \text { MDate } & 12.7956 & -0.3012 \\ 2 & \text { Residual } & -0.3012 & 0.5178\end{array}$

\section{Fit Statistics}

$\begin{array}{ll}-2 \text { Res Log Likelihood } & 803.2 \\ \text { AIC (smaller is better) } & 807.2 \\ \text { AICC (smaller is better) } & 807.3 \\ \text { BIC (smaller is better) } & 812.2\end{array}$

Null Model Likelihood Ratio Test

DF Chi-Square $\quad \mathrm{Pr}>$ ChiSq

$1 \quad 82.87 \quad<.0001$

The SAS System 10:41 Thursday, March 14, 201386

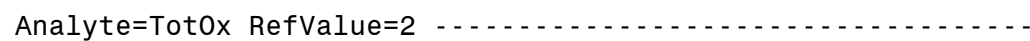

The Mixed Procedure

Solution for Fixed Effects

\begin{tabular}{|c|c|c|c|c|c|}
\hline & & tandard & & & \\
\hline Effect & Estimate & Error & DF & $\mathrm{t}$ Value & $\operatorname{Pr}>|t|$ \\
\hline cept & 4.3861 & 0.5166 & 90.9 & 8.49 & $<.00$ \\
\hline
\end{tabular}

Solution for Random Effects

Std Err 
SRNL-STI-2013-00139

Revision 0

\begin{tabular}{|c|c|c|c|c|c|c|}
\hline Effect & MDate & Estimate & Pred & DF & t Value & $\operatorname{Pr}>|t|$ \\
\hline MDate & 19298 & -1.5954 & 1.8734 & 92 & -0.85 & 0.3966 \\
\hline MDate & 19300 & 1.3651 & 1.8734 & 92 & 0.73 & 0.4680 \\
\hline MDate & 19302 & -0.3266 & 1.8734 & 92 & -0.17 & 0.8620 \\
\hline MDate & 19304 & -3.2871 & 1.8734 & 92 & -1.75 & 0.0827 \\
\hline MDate & 19305 & -2.0183 & 1.8734 & 92 & -1.08 & 0.2841 \\
\hline MDate & 19306 & -2.4412 & 1.8734 & 92 & -1.30 & 0.1958 \\
\hline MDate & 19307 & -2.0183 & 1.8734 & 92 & -1.08 & 0.2841 \\
\hline MDate & 19310 & 0.5193 & 1.8734 & 92 & 0.28 & 0.7823 \\
\hline MDate & 19311 & 0.5193 & 1.8734 & 92 & 0.28 & 0.7823 \\
\hline MDate & 19312 & -0.3266 & 1.8734 & 92 & -0.17 & 0.8620 \\
\hline MDate & 19313 & 0.1044 & 1.4174 & 88.2 & 0.07 & 0.9415 \\
\hline MDate & 19314 & 0.5193 & 1.8734 & 92 & 0.28 & 0.7823 \\
\hline MDate & 19315 & 0.09634 & 1.8734 & 92 & 0.05 & 0.9591 \\
\hline MDate & 19316 & -1.1724 & 1.8734 & 92 & -0.63 & 0.5330 \\
\hline MDate & 19317 & -1.1724 & 1.8734 & 92 & -0.63 & 0.5330 \\
\hline MDate & 19318 & -0.3539 & 1.4174 & 88.2 & -0.25 & 0.8034 \\
\hline MDate & 19319 & 0.09634 & 1.8734 & 92 & 0.05 & 0.9591 \\
\hline MDate & 19321 & 0.5787 & 1.2072 & 92 & 0.48 & 0.6328 \\
\hline MDate & 19322 & 0.09634 & 1.8734 & 92 & 0.05 & 0.9591 \\
\hline MDate & 19323 & 0.1044 & 1.4174 & 88.2 & 0.07 & 0.9415 \\
\hline MDate & 19324 & 0.5193 & 1.8734 & 92 & 0.28 & 0.7823 \\
\hline MDate & 19325 & 0.2645 & 1.2072 & 92 & 0.22 & 0.8271 \\
\hline MDate & 19326 & -0.7495 & 1.8734 & 92 & -0.40 & 0.6900 \\
\hline MDate & 19327 & 0.09634 & 1.8734 & 92 & 0.05 & 0.9591 \\
\hline MDate & 19329 & 1.7082 & 1.4174 & 88.2 & 1.21 & 0.2313 \\
\hline MDate & 19330 & 2.1665 & 1.4174 & 88.2 & 1.53 & 0.1300 \\
\hline MDate & 19331 & 0.09634 & 1.8734 & 92 & 0.05 & 0.9591 \\
\hline MDate & 19333 & 1.7082 & 1.4174 & 88.2 & 1.21 & 0.2313 \\
\hline MDate & 19334 & 1.7880 & 1.8734 & 92 & 0.95 & 0.3424 \\
\hline MDate & 19335 & -0.3266 & 1.8734 & 92 & -0.17 & 0.8620 \\
\hline MDate & 19336 & -0.7495 & 1.8734 & 92 & -0.40 & 0.6900 \\
\hline MDate & 19337 & 2.2110 & 1.8734 & 92 & 1.18 & 0.2410 \\
\hline MDate & 19338 & -0.7495 & 1.8734 & 92 & -0.40 & 0.6900 \\
\hline MDate & 19339 & 0.3335 & 1.4174 & 88.2 & 0.24 & 0.8145 \\
\hline MDate & 19340 & -1.0412 & 1.4174 & 88.2 & -0.73 & 0.4645 \\
\hline MDate & 19341 & -6.6489 & 1.2072 & 92 & -5.51 & $<.0001$ \\
\hline
\end{tabular}

The SAS System

10:41 Thursday, March 14, 201387

Analyte $=$ Tot0 $x$ RefValue $=2$

The Mixed Procedure

Solution for Random Effects

$\begin{array}{lrrrrrr}\text { Effect } & \text { MDate } & \text { Estimate } & \begin{array}{r}\text { Std Err } \\ \text { Pred }\end{array} & \text { DF } & \text { t Value } & \text { Pr }>|t| \\ \text { MDate } & 19342 & -6.1775 & 1.2072 & 92 & -5.12 & <.0001 \\ \text { MDate } & 19343 & -4.5558 & 1.8734 & 92 & -2.43 & 0.0170 \\ \text { MDate } & 19344 & -4.1329 & 1.8734 & 92 & -2.21 & 0.0299 \\ \text { MDate } & 19347 & -1.9577 & 1.4174 & 88.2 & -1.38 & 0.1707 \\ \text { MDate } & 19348 & -2.6451 & 1.4174 & 88.2 & -1.87 & 0.0653 \\ \text { MDate } & 19350 & -5.8687 & 1.0822 & 97.3 & -5.42 & <.0001 \\ \text { MDate } & 19351 & -8.3621 & 1.8734 & 92 & -4.46 & <.0001\end{array}$


SRNL-STI-2013-00139

Revision 0

\begin{tabular}{|c|c|c|c|c|c|c|}
\hline MDate & 19352 & -7.5163 & 1.8734 & 92 & -4.01 & 0.0001 \\
\hline MDate & 19354 & -3.2871 & 1.8734 & 92 & -1.75 & 0.0827 \\
\hline MDate & 19355 & -2.8641 & 1.8734 & 92 & -1.53 & 0.1297 \\
\hline MDate & 19356 & -2.8641 & 1.8734 & 92 & -1.53 & 0.1297 \\
\hline MDate & 19357 & -2.8641 & 1.8734 & 92 & -1.53 & 0.1297 \\
\hline MDate & 19358 & -3.3324 & 1.4174 & 88.2 & -2.35 & 0.0209 \\
\hline MDate & 19359 & -3.1033 & 1.4174 & 88.2 & -2.19 & 0.0312 \\
\hline MDate & 19360 & -2.8641 & 1.8734 & 92 & -1.53 & 0.1297 \\
\hline MDate & 19361 & -4.4780 & 1.4174 & 88.2 & -3.16 & 0.0022 \\
\hline MDate & 19362 & -3.2871 & 1.8734 & 92 & -1.75 & 0.0827 \\
\hline MDate & 19363 & -4.7072 & 1.4174 & 88.2 & -3.32 & 0.0013 \\
\hline MDate & 19364 & -4.1329 & 1.8734 & 92 & -2.21 & 0.0299 \\
\hline MDate & 19365 & -4.1329 & 1.8734 & 92 & -2.21 & 0.0299 \\
\hline MDate & 19366 & 4.2286 & 1.4174 & 88.2 & 2.98 & 0.0037 \\
\hline MDate & 19367 & 5.1714 & 1.8734 & 92 & 2.76 & 0.0070 \\
\hline MDate & 19368 & 6.0173 & 1.8734 & 92 & 3.21 & 0.0018 \\
\hline MDate & 19369 & 2.6247 & 1.4174 & 88.2 & 1.85 & 0.0674 \\
\hline MDate & 19370 & 0.09634 & 1.8734 & 92 & 0.05 & 0.9591 \\
\hline MDate & 19371 & 2.9356 & 1.2072 & 92 & 2.43 & 0.0170 \\
\hline MDate & 19372 & 4.8911 & 1.0822 & 97.3 & 4.52 & $<.0001$ \\
\hline MDate & 19373 & 8.1319 & 1.8734 & 92 & 4.34 & $<.0001$ \\
\hline MDate & 19374 & 11.3314 & 1.4174 & 88.2 & 7.99 & $<.0001$ \\
\hline MDate & 19375 & 8.3528 & 1.4174 & 88.2 & 5.89 & $<.0001$ \\
\hline MDate & 19376 & 13.2070 & 1.8734 & 92 & 7.05 & $<.0001$ \\
\hline MDate & 19377 & 7.6654 & 1.4174 & 88.2 & 5.41 & $<.0001$ \\
\hline MDate & 19378 & 9.4984 & 1.4174 & 88.2 & 6.70 & $<.0001$ \\
\hline MDate & 19379 & 9.0401 & 1.4174 & 88.2 & 6.38 & $<.0001$ \\
\hline MDate & 19380 & 9.7275 & 1.4174 & 88.2 & 6.86 & $<.0001$ \\
\hline MDate & 19381 & 10.4149 & 1.4174 & 88.2 & 7.35 & $<.0001$ \\
\hline MDate & 19382 & 8.8110 & 1.4174 & 88.2 & 6.22 & $<.0001$ \\
\hline MDate & 19383 & -1.2703 & 1.4174 & 88.2 & -0.90 & 0.3726 \\
\hline MDate & 19384 & -4.0389 & 0.9979 & 103 & -4.05 & 0.0001 \\
\hline MDate & 19385 & -3.2871 & 1.8734 & 92 & -1.75 & 0.0827 \\
\hline MDate & 19386 & -3.6635 & 1.2072 & 92 & -3.03 & 0.0031 \\
\hline MDate & 19387 & -0.3266 & 1.8734 & 92 & -0.17 & 0.8620 \\
\hline MDate & 19388 & -1.1724 & 1.8734 & 92 & -0.63 & 0.5330 \\
\hline MDate & 19389 & -3.2871 & 1.8734 & 92 & -1.75 & 0.0827 \\
\hline
\end{tabular}

The SAS System

10:41 Thursday, March 14, 201388

Analyte $=$ Tot0 $x$ RefValue $=2$

The Mixed Procedure

Solution for Random Effects

$\begin{array}{lrrrrrr}\text { Effect } & \text { MDate } & \text { Estimate } & \begin{array}{r}\text { Std Err } \\ \text { Pred }\end{array} & \text { DF } & t \text { Value } & \text { Pr }>|t| \\ & & & & & & \\ \text { MDate } & 19390 & -3.2385 & 1.0822 & 97.3 & -2.99 & 0.0035 \\ \text { MDate } & 19391 & -1.5954 & 1.8734 & 92 & -0.85 & 0.3966 \\ \text { MDate } & 19392 & -1.1724 & 1.8734 & 92 & -0.63 & 0.5330 \\ \text { MDate } & 19393 & -0.8121 & 1.4174 & 88.2 & -0.57 & 0.5681 \\ \text { MDate } & 19394 & -0.5830 & 1.4174 & 88.2 & -0.41 & 0.6818 \\ \text { MDate } & 19395 & 1.3651 & 1.8734 & 92 & 0.73 & 0.4680 \\ \text { MDate } & 19396 & 0.3335 & 1.4174 & 88.2 & 0.24 & 0.8145 \\ \text { MDate } & 19397 & -2.8641 & 1.8734 & 92 & -1.53 & 0.1297\end{array}$


SRNL-STI-2013-00139

Revision 0

$\begin{array}{lllllll}\text { MDate } & 19398 & -2.5637 & 1.2072 & 92 & -2.12 & 0.0364 \\ \text { MDate } & 19399 & -0.7495 & 1.8734 & 92 & -0.40 & 0.6900\end{array}$

The SAS System

10:41 Thursday, March 14, 201389

Analyte $=$ Tot0 $x$ RefValue $=16$

The Mixed Procedure

Model Information

Data Set

Dependent Variable

Covariance Structure

Estimation Method

Residual Variance Method

Fixed Effects SE Method

Degrees of Freedom Method
WORK. TOMSORT

PctDiff

Variance Components

REML

Profile

Prasad-Rao-Jeske-

Kackar-Harville

Kenward-Roger

Class Level Information

\begin{tabular}{|c|c|c|c|c|c|c|}
\hline Class & Levels & Values & & & & \\
\hline MDate & 90 & 19298 & 19300 & 19302 & 19304 & 19305 \\
\hline & & 19306 & 19307 & 19310 & 19311 & 19312 \\
\hline & & 19313 & 19314 & 19315 & 19316 & 19317 \\
\hline & & 19318 & 19319 & 19321 & 19322 & 19323 \\
\hline & & 19324 & 19325 & 19326 & 19327 & 19329 \\
\hline & & 19330 & 19331 & 19333 & 19334 & 19335 \\
\hline & & 19336 & 19337 & 19338 & 19339 & 19340 \\
\hline & & 19341 & 19342 & 19343 & 19344 & 19347 \\
\hline & & 19348 & 19350 & 19351 & 19352 & 19354 \\
\hline & & 19355 & 19356 & 19357 & 19358 & 19359 \\
\hline & & 19360 & 19361 & 19362 & 19363 & 19364 \\
\hline & & 19365 & 19366 & 19367 & 19368 & 19369 \\
\hline & & 19370 & 19371 & 19372 & 19373 & 19374 \\
\hline & & 19375 & 19376 & 19377 & 19378 & 19379 \\
\hline & & 19380 & 19381 & 19382 & 19383 & 19384 \\
\hline & & 19385 & 19386 & 19387 & 19388 & 19389 \\
\hline & & 19390 & 19391 & 19392 & 19393 & 19394 \\
\hline & & 19395 & 19396 & 19397 & 19398 & 19399 \\
\hline
\end{tabular}

Dimensions

Covariance Parameters

2

Columns in $X$

Columns in $Z$

1

Subjects

90

Max Obs Per Subject

Observations Used

Observations Not Used

Total Observations

0

145

The SAS System

10:41 Thursday, March 14, 201390 
The Mixed Procedure

$$
\text { Iteration History }
$$

$\begin{array}{rrrr}\text { Iteration } & \text { Evaluations } & -2 \text { Res Log Like } & \text { Criterion } \\ 0 & 1 & 870.62961936 & \\ 1 & 2 & 826.08398746 & 0.02872588 \\ 2 & 1 & 815.67595604 & 0.01239290 \\ 3 & 1 & 811.46988795 & 0.00284906 \\ 4 & 1 & 810.57843749 & 0.00019563 \\ 5 & 1 & 810.52251294 & 0.00000111 \\ 6 & 1 & 810.52221004 & 0.00000000\end{array}$

Convergence criteria met.

Covariance Parameter Estimates

$\begin{array}{lrrrr}\text { Cov Parm } & \text { Estimate } & \text { Alpha } & \text { Lower } & \text { Upper } \\ & & & & \\ \text { MDate } & 25.5001 & 0.05 & 17.0246 & 33.9756 \\ \text { Residual } & 3.4231 & 0.05 & 2.4133 & 5.2346\end{array}$

Asymptotic Covariance Matrix of Estimates

$\begin{array}{rlrr}\text { Row } & \text { Cov Parm } & \text { CovP1 } & \text { CovP2 } \\ & & & \\ 1 & \text { MDate } & 18.6997 & -0.4503 \\ 2 & \text { Residual } & -0.4503 & 0.4486\end{array}$

Fit Statistics

$\begin{array}{ll}\text {-2 Res Log Likelihood } & 810.5 \\ \text { AIC (smaller is better) } & 814.5 \\ \text { AICC (smaller is better) } & 814.6 \\ \text { BIC (smaller is better) } & 819.5\end{array}$

Null Model Likelihood Ratio Test

\begin{tabular}{|c|c|c|}
\hline DF & Chi-Square & $\mathrm{Pr}>\mathrm{ChiSq}$ \\
\hline 1 & 60.11 & $<.0001$ \\
\hline
\end{tabular}

The SAS System 10:41 Thursday, March 14, 201391 
SRNL-STI-2013-00139

Revision 0

Solution for Fixed Effects

$\begin{array}{lrrrrr}\text { Effect } & \text { Estimate } & \begin{array}{r}\text { Standard } \\ \text { Error }\end{array} & \text { DF } & t \text { Value } & \text { Pr }>|t| \\ \text { Intercept } & 0.4386 & 0.5584 & 86 & 0.79 & 0.4343\end{array}$

Solution for Random Effects

\begin{tabular}{|c|c|c|c|c|c|c|}
\hline \multirow{2}{*}{ Effect } & \multicolumn{5}{|c|}{ Std Err } & \multirow[b]{2}{*}{$\operatorname{Pr}>|t|$} \\
\hline & MDate & Estimate & Pred & DF & t Value & \\
\hline MDate & 19298 & -1.0480 & 1.8189 & 82.3 & -0.58 & 0.5661 \\
\hline MDate & 19300 & -0.9929 & 1.8189 & 82.3 & -0.55 & 0.5866 \\
\hline MDate & 19302 & -0.6071 & 1.8189 & 82.3 & -0.33 & 0.7394 \\
\hline MDate & 19304 & -2.2051 & 1.8189 & 82.3 & -1.21 & 0.2288 \\
\hline MDate & 19305 & -1.5439 & 1.8189 & 82.3 & -0.85 & 0.398 \\
\hline MDate & 19306 & -1.6541 & 1.8189 & 82.3 & -0.91 & 0.365 \\
\hline MDate & 19307 & -1.6541 & 1.8189 & 82.3 & -0.91 & 0.365 \\
\hline MDate & 19310 & -1.4337 & 1.8189 & 82.3 & -0.79 & 0.432 \\
\hline MDate & 19311 & -1.2133 & 1.8189 & 82.3 & -0.67 & 0.50 \\
\hline MDate & 19312 & -1.6541 & 1.8189 & 82.3 & -0.91 & 0.365 \\
\hline MDate & 19313 & 0.08678 & 1.3756 & 84.2 & 0.06 & 0.94 \\
\hline MDate & 19314 & 1.4317 & 1.8189 & 82.3 & 0.79 & 0.433 \\
\hline MDate & 19315 & -0.8276 & 1.8189 & 82.3 & -0.45 & 0.650 \\
\hline MDate & 19316 & -1.5990 & 1.8189 & 82.3 & -0.88 & 0.381 \\
\hline MDate & 19317 & 2.0378 & 1.8189 & 82.3 & 1.12 & 0.265 \\
\hline MDate & 19318 & -2.1096 & 1.3756 & 84.2 & -1.53 & $0.12 \varepsilon$ \\
\hline MDate & 19319 & -0.9929 & 1.8189 & 82.3 & -0.55 & 0.586 \\
\hline MDate & 19321 & 0.9162 & 1.1768 & 91.4 & 0.78 & 0.438 \\
\hline MDate & 19322 & 2.0929 & 1.8189 & 82.3 & 1.15 & 0.253 \\
\hline MDate & 19323 & -2.1681 & 1.3756 & 84.2 & -1.58 & 0.118 \\
\hline MDate & 19324 & -0.1112 & 1.8189 & 82.3 & -0.06 & 0.95 \\
\hline MDate & 19325 & -0.6193 & 1.1768 & 91.4 & -0.53 & 0.600 \\
\hline MDate & 19326 & -0.05611 & 1.8189 & 82.3 & -0.03 & 0.975 \\
\hline MDate & 19327 & -3.6929 & 1.8189 & 82.3 & -2.03 & 0.04 \\
\hline MDate & 19329 & -0.2646 & 1.3756 & 84.2 & -0.19 & 0.847 \\
\hline MDate & 19330 & 2.1953 & 1.3756 & 84.2 & 1.60 & 0.114 \\
\hline MDate & 19331 & -2.0949 & 1.8189 & 82.3 & -1.15 & 0.252 \\
\hline MDate & 19333 & 1.8438 & 1.3756 & 84.2 & 1.34 & 0.183 \\
\hline MDate & 19334 & -0.1112 & 1.8189 & 82.3 & -0.06 & 0.951 \\
\hline MDate & 19335 & -1.3786 & 1.8189 & 82.3 & -0.76 & 0.450 \\
\hline MDate & 19336 & -0.5520 & 1.8189 & 82.3 & -0.30 & 0.762 \\
\hline MDate & 19337 & -1.2133 & 1.8189 & 82.3 & -0.67 & 0.506 \\
\hline MDate & 19338 & -0.3867 & 1.8189 & 82.3 & -0.21 & 0.832 \\
\hline MDate & 19339 & -0.6746 & 1.3756 & 84.2 & -0.49 & 0.625 \\
\hline MDate & 19340 & -0.4403 & 1.3756 & 84.2 & -0.32 & 0.749 \\
\hline MDate & 19341 & -1.1377 & 1.1768 & 91.4 & -0.97 & 0.3362 \\
\hline
\end{tabular}

The SAS System

10:41 Thursday, March 14, 201392

Analyte=Tot0x RefValue $=16$

The Mixed Procedure

Solution for Random Effects 
SRNL-STI-2013-00139

Revision 0

\begin{tabular}{|c|c|c|c|c|c|c|}
\hline & & & Std Err & & & \\
\hline Effect & MDate & Estimate & Pred & DF & t Value & $\operatorname{Pr}>|t|$ \\
\hline MDate & 19342 & -0.3999 & 1.1768 & 91.4 & -0.34 & 0.7348 \\
\hline MDate & 19343 & -0.2765 & 1.8189 & 82.3 & -0.15 & 0.8795 \\
\hline MDate & 19344 & -0.2765 & 1.8189 & 82.3 & -0.15 & 0.8795 \\
\hline MDate & 19347 & 1.3460 & 1.3756 & 84.2 & 0.98 & 0.3306 \\
\hline MDate & 19348 & -1.3482 & 1.3756 & 84.2 & -0.98 & 0.3299 \\
\hline MDate & 19350 & -4.7934 & 1.0602 & 99.5 & -4.52 & $<.0001$ \\
\hline MDate & 19351 & -7.7705 & 1.8189 & 82.3 & -4.27 & $<.0001$ \\
\hline MDate & 19352 & -9.2032 & 1.8189 & 82.3 & -5.06 & $<.0001$ \\
\hline MDate & 19354 & -0.4418 & 1.8189 & 82.3 & -0.24 & 0.8087 \\
\hline MDate & 19355 & -0.2214 & 1.8189 & 82.3 & -0.12 & 0.9034 \\
\hline MDate & 19356 & -0.1663 & 1.8189 & 82.3 & -0.09 & 0.9274 \\
\hline MDate & 19357 & 0.1643 & 1.8189 & 82.3 & 0.09 & 0.9282 \\
\hline MDate & 19358 & 0.02821 & 1.3756 & 84.2 & 0.02 & 0.9837 \\
\hline MDate & 19359 & 0.2625 & 1.3756 & 84.2 & 0.19 & 0.8491 \\
\hline MDate & 19360 & 0.3296 & 1.8189 & 82.3 & 0.18 & 0.8566 \\
\hline MDate & 19361 & -1.4653 & 1.3756 & 84.2 & -1.07 & 0.2898 \\
\hline MDate & 19362 & -1.9296 & 1.8189 & 82.3 & -1.06 & 0.2918 \\
\hline MDate & 19363 & -1.4067 & 1.3756 & 84.2 & -1.02 & 0.3094 \\
\hline MDate & 19364 & -1.4337 & 1.8189 & 82.3 & -0.79 & 0.4328 \\
\hline MDate & 19365 & -0.4418 & 1.8189 & 82.3 & -0.24 & 0.8087 \\
\hline MDate & 19366 & -0.6453 & 1.3756 & 84.2 & -0.47 & 0.6402 \\
\hline MDate & 19367 & 3.6909 & 1.8189 & 82.3 & 2.03 & 0.0457 \\
\hline MDate & 19368 & -0.8827 & 1.8189 & 82.3 & -0.49 & 0.6288 \\
\hline MDate & 19369 & 2.2245 & 1.3756 & 84.2 & 1.62 & 0.1096 \\
\hline MDate & 19370 & 3.8562 & 1.8189 & 82.3 & 2.12 & 0.0370 \\
\hline MDate & 19371 & 1.5942 & 1.1768 & 91.4 & 1.35 & 0.1789 \\
\hline MDate & 19372 & 4.4586 & 1.0602 & 99.5 & 4.21 & $<.0001$ \\
\hline MDate & 19373 & 4.1868 & 1.8189 & 82.3 & 2.30 & 0.0239 \\
\hline MDate & 19374 & 3.9523 & 1.3756 & 84.2 & 2.87 & 0.0051 \\
\hline MDate & 19375 & 4.6552 & 1.3756 & 84.2 & 3.38 & 0.0011 \\
\hline MDate & 19376 & 34.1078 & 1.8189 & 82.3 & 18.75 & $<.0001$ \\
\hline MDate & 19377 & 7.3786 & 1.3756 & 84.2 & 5.36 & $<.0001$ \\
\hline MDate & 19378 & 7.7007 & 1.3756 & 84.2 & 5.60 & $<.0001$ \\
\hline MDate & 19379 & 6.2951 & 1.3756 & 84.2 & 4.58 & $<.0001$ \\
\hline MDate & 19380 & 6.1487 & 1.3756 & 84.2 & 4.47 & $<.0001$ \\
\hline MDate & 19381 & 6.7344 & 1.3756 & 84.2 & 4.90 & $<.0001$ \\
\hline MDate & 19382 & 8.3450 & 1.3756 & 84.2 & 6.07 & $<.0001$ \\
\hline MDate & 19383 & 0.4675 & 1.3756 & 84.2 & 0.34 & 0.7348 \\
\hline MDate & 19384 & -0.5367 & 0.9825 & 107 & -0.55 & 0.5860 \\
\hline MDate & 19385 & -0.2214 & 1.8189 & 82.3 & -0.12 & 0.9034 \\
\hline MDate & 19386 & -0.3401 & 1.1768 & 91.4 & -0.29 & 0.7732 \\
\hline MDate & 19387 & -3.0317 & 1.8189 & 82.3 & -1.67 & 0.099 \\
\hline MDate & 19388 & -6.8889 & 1.8189 & 82.3 & -3.79 & 0.0003 \\
\hline MDate & 19389 & -5.8970 & 1.8189 & 82.3 & -3.24 & 0.001 \\
\hline
\end{tabular}

The SAS System

10:41 Thursday, March 14, 201393

Analyte=Tot0x RefValue $=16$

The Mixed Procedure

Solution for Random Effects 
SRNL-STI-2013-00139

Revision 0

$\begin{array}{lcccccc}\text { Effect } & \text { MDate } & \text { Estimate } & \begin{array}{r}\text { Std Err } \\ \text { Pred }\end{array} & \text { DF } & \text { t Value } & \text { Pr }>|t| \\ \text { MDate } & 19390 & -3.6747 & 1.0602 & 99.5 & -3.47 & 0.0008 \\ \text { MDate } & 19391 & -5.1256 & 1.8189 & 82.3 & -2.82 & 0.0060 \\ \text { MDate } & 19392 & -2.5909 & 1.8189 & 82.3 & -1.42 & 0.1581 \\ \text { MDate } & 19393 & -2.6074 & 1.3756 & 84.2 & -1.90 & 0.0615 \\ \text { MDate } & 19394 & -3.9545 & 1.3756 & 84.2 & -2.87 & 0.0051 \\ \text { MDate } & 19395 & -5.1256 & 1.8189 & 82.3 & -2.82 & 0.0060 \\ \text { MDate } & 19396 & -1.8753 & 1.3756 & 84.2 & -1.36 & 0.1764 \\ \text { MDate } & 19397 & -2.7562 & 1.8189 & 82.3 & -1.52 & 0.1335 \\ \text { MDate } & 19398 & -3.8298 & 1.1768 & 91.4 & -3.25 & 0.0016 \\ \text { MDate } & 19399 & -2.5358 & 1.8189 & 82.3 & -1.39 & 0.1670\end{array}$


SRNL-STI-2013-00139

Revision 0

\section{Screened Measurements}

The SAS System

09:55 Thursday, March 14, 20131

Analyte=Formate RefValue=2 $\ldots \ldots \ldots \ldots$

The Mixed Procedure

Model Information

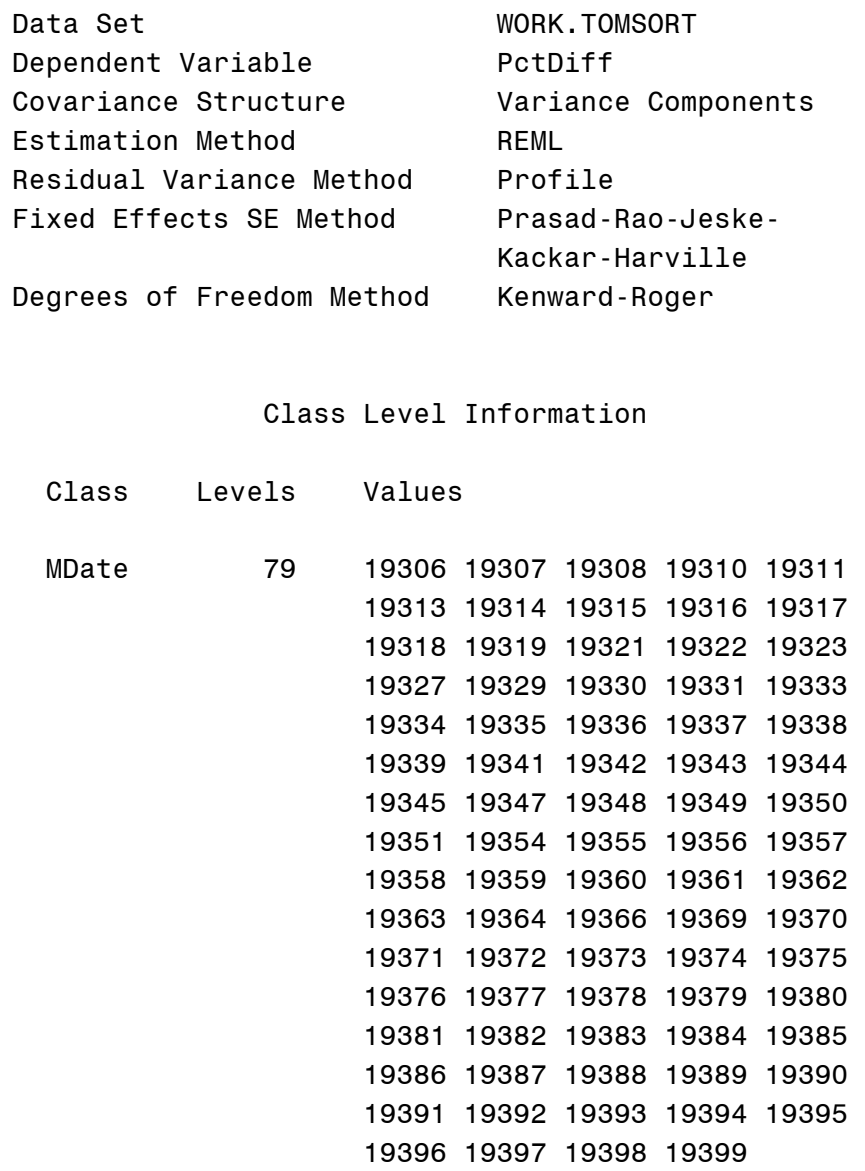

Dimensions

Covariance Parameters

2

Columns in $X$

Columns in $Z$

Subjects

9

Max Obs Per Subject

1

Observations Used

Observations Not Used

156

Total Observations

156

0

156 
SRNL-STI-2013-00139

Revision 0

Analyte=Formate RefValue $=2$

The Mixed Procedure

Iteration History

$\begin{array}{rrrr}\text { Iteration } & \text { Evaluations } & -2 \text { Res Log Like } & \text { Criterion } \\ 0 & 1 & 725.17061574 & \\ 1 & 3 & 691.56933896 & 0.01146317 \\ 2 & 2 & 690.01983597 & 0.00024085 \\ 3 & 1 & 689.97400134 & 0.00000173 \\ 4 & 1 & 689.97364935 & 0.00000000\end{array}$

Convergence criteria met.

Covariance Parameter Estimates

$\begin{array}{lrrrr}\text { Cov Parm } & \text { Estimate } & \text { Alpha } & \text { Lower } & \text { Upper } \\ & & & & \\ \text { MDate } & 2.8576 & 0.05 & 1.4356 & 4.2797 \\ \text { Residual } & 2.9201 & 0.05 & 2.2107 & 4.0377\end{array}$

Asymptotic Covariance Matrix of Estimates

$\begin{array}{rlrr}\text { Row } & \text { Cov Parm } & \text { CovP1 } & \text { CovP2 } \\ & & & \\ 1 & \text { MDate } & 0.5264 & -0.08596 \\ 2 & \text { Residual } & -0.08596 & 0.1991\end{array}$

Fit Statistics

$\begin{array}{ll}\text {-2 Res Log Likelihood } & 690.0 \\ \text { AIC (smaller is better) } & 694.0 \\ \text { AICC (smaller is better) } & 694.1 \\ \text { BIC (smaller is better) } & 698.7\end{array}$

Null Model Likelihood Ratio Test

\begin{tabular}{|c|c|c|}
\hline DF & Chi-Square & $\mathrm{Pr}>\mathrm{ChiSq}$ \\
\hline 1 & 35.20 & $<.0001$ \\
\hline
\end{tabular}

The SAS System

09:55 Thursday, March 14, 2013

Analyte=Formate RefValue $=2$

The Mixed Procedure 
SRNL-STI-2013-00139

Revision 0

Solution for Fixed Effects

$\begin{array}{lrrrrr}\text { Effect } & \text { Estimate } & \begin{array}{r}\text { Standard } \\ \text { Error }\end{array} & \text { DF } & t \text { Value } & \operatorname{Pr}>|t| \\ \text { Intercept } & 2.5432 & 0.2425 & 81.2 & 10.49 & <.0001\end{array}$

Solution for Random Effects

\begin{tabular}{|c|c|c|c|c|c|c|}
\hline & & & Std Err & & & \\
\hline Effect & MDate & Estimate & Pred & DF & t Value & $\operatorname{Pr}>|t|$ \\
\hline MDate & 19306 & 0.9678 & 1.2392 & 114 & 0.78 & 0.4364 \\
\hline MDate & 19307 & -0.02136 & 1.2392 & 114 & -0.02 & 0.986 \\
\hline MDate & 19308 & -0.5159 & 1.2392 & 114 & -0.42 & 0.6779 \\
\hline MDate & 19310 & -0.3595 & 1.0190 & 151 & -0.35 & 0.7247 \\
\hline MDate & 19311 & -0.7632 & 1.2392 & 114 & -0.62 & 0.539 \\
\hline MDate & 19313 & -0.1940 & 1.0190 & 151 & -0.19 & 0.849 \\
\hline MDate & 19314 & -1.0105 & 1.2392 & 114 & -0.82 & 0.41 \\
\hline MDate & 19315 & -0.7632 & 1.2392 & 114 & -0.62 & 0.53 \\
\hline MDate & 19316 & -0.5159 & 1.2392 & 114 & -0.42 & 0.677 \\
\hline MDate & 19317 & -0.5159 & 1.2392 & 114 & -0.42 & 0.677 \\
\hline MDate & 19318 & -1.3997 & 0.8880 & 155 & -1.58 & 0.117 \\
\hline MDate & 19319 & -2.4943 & 1.2392 & 114 & -2.01 & 0.046 \\
\hline MDate & 19321 & -2.1457 & 0.8880 & 155 & -2.42 & 0.016 \\
\hline MDate & 19322 & -2.5186 & 0.8880 & 155 & -2.84 & 0.005 \\
\hline MDate & 19323 & 3.9424 & 1.0190 & 151 & 3.87 & 0.000 \\
\hline MDate & 19327 & 1.9570 & 1.2392 & 114 & 1.58 & 0.117 \\
\hline MDate & 19329 & -0.02858 & 1.0190 & 151 & -0.03 & 0.977 \\
\hline MDate & 19330 & 0.6333 & 1.0190 & 151 & 0.62 & 0.535 \\
\hline MDate & 19331 & -1.1868 & 1.0190 & 151 & -1.16 & 0.246 \\
\hline MDate & 19333 & 0.2259 & 1.2392 & 114 & 0.18 & 0.855 \\
\hline MDate & 19334 & -2.7416 & 1.2392 & 114 & -2.21 & 0.028 \\
\hline MDate & 19335 & 0.4732 & 1.2392 & 114 & 0.38 & 0.703 \\
\hline MDate & 19336 & -0.02858 & 1.0190 & 151 & -0.03 & 0.977 \\
\hline MDate & 19337 & 0.2259 & 1.2392 & 114 & 0.18 & 0.855 \\
\hline MDate & 19338 & -2.2470 & 1.2392 & 114 & -1.81 & 0.072 \\
\hline MDate & 19339 & -1.7524 & 1.2392 & 114 & -1.41 & 0.160 \\
\hline MDate & 19341 & 0.6333 & 1.0190 & 151 & 0.62 & 0.535 \\
\hline MDate & 19342 & -0.7781 & 0.8880 & 155 & -0.88 & 0.382 \\
\hline MDate & 19343 & -0.02136 & 1.2392 & 114 & -0.02 & 0.986 \\
\hline MDate & 19344 & -1.5241 & 0.8880 & 155 & -1.72 & 0.088 \\
\hline MDate & 19345 & -0.7632 & 1.2392 & 114 & -0.62 & 0.539 \\
\hline MDate & 19347 & 0.4678 & 1.0190 & 151 & 0.46 & 0.646 \\
\hline MDate & 19348 & -0.3595 & 1.0190 & 151 & -0.35 & 0.724 \\
\hline MDate & 19349 & -0.02136 & 1.2392 & 114 & -0.02 & 0.986 \\
\hline MDate & 19350 & -0.1340 & 0.7996 & 151 & -0.17 & 0.8672 \\
\hline MDate & 19351 & -0.5250 & 1.0190 & 151 & -0.52 & 0.6072 \\
\hline
\end{tabular}

The SAS System

09:55 Thursday, March 14, 20134

Analyte=Formate RefValue $=2$

The Mixed Procedure

Solution for Random Effects 
SRNL-STI-2013-00139

Revision 0

\begin{tabular}{|c|c|c|c|c|c|c|}
\hline & & & Std Err & & & \\
\hline Effect & MDate & Estimate & Pred & DF & t Value & $\operatorname{Pr}>|t|$ \\
\hline MDate & 19354 & 0.4651 & 0.8880 & 155 & 0.52 & 0.6012 \\
\hline MDate & 19355 & 0.3023 & 1.0190 & 151 & 0.30 & 0.7671 \\
\hline MDate & 19356 & 0.2164 & 0.8880 & 155 & 0.24 & 0.8078 \\
\hline MDate & 19357 & 0.2259 & 1.2392 & 114 & 0.18 & 0.8557 \\
\hline MDate & 19358 & -0.3595 & 1.0190 & 151 & -0.35 & 0.7247 \\
\hline MDate & 19359 & 0.3023 & 1.0190 & 151 & 0.30 & 0.7671 \\
\hline MDate & 19360 & 0.7205 & 1.2392 & 114 & 0.58 & 0.5621 \\
\hline MDate & 19361 & -0.3595 & 1.0190 & 151 & -0.35 & 0.7247 \\
\hline MDate & 19362 & -0.7632 & 1.2392 & 114 & -0.62 & 0.5392 \\
\hline MDate & 19363 & 0.3023 & 1.0190 & 151 & 0.30 & 0.7671 \\
\hline MDate & 19364 & -1.2578 & 1.2392 & 114 & -1.02 & 0.3122 \\
\hline MDate & 19366 & -1.0105 & 1.2392 & 114 & -0.82 & 0.4165 \\
\hline MDate & 19369 & 0.4732 & 1.2392 & 114 & 0.38 & 0.7033 \\
\hline MDate & 19370 & -1.2578 & 1.2392 & 114 & -1.02 & 0.3122 \\
\hline MDate & 19371 & -1.2754 & 0.8880 & 155 & -1.44 & 0.1529 \\
\hline MDate & 19372 & -3.0250 & 0.7352 & 147 & -4.11 & $<.0001$ \\
\hline MDate & 19373 & 0.4732 & 1.2392 & 114 & 0.38 & 0.7033 \\
\hline MDate & 19374 & 0.2259 & 1.2392 & 114 & 0.18 & 0.8557 \\
\hline MDate & 19375 & 1.2151 & 1.2392 & 114 & 0.98 & 0.3289 \\
\hline MDate & 19376 & 2.7872 & 0.7352 & 147 & 3.79 & 0.0002 \\
\hline MDate & 19377 & 2.0812 & 0.8880 & 155 & 2.34 & 0.0204 \\
\hline MDate & 19378 & 1.2151 & 1.2392 & 114 & 0.98 & 0.3289 \\
\hline MDate & 19379 & 2.0812 & 0.8880 & 155 & 2.34 & 0.0204 \\
\hline MDate & 19380 & 0.8380 & 0.8880 & 155 & 0.94 & 0.3468 \\
\hline MDate & 19381 & -0.5322 & 0.7996 & 151 & -0.67 & 0.5067 \\
\hline MDate & 19382 & -0.02858 & 1.0190 & 151 & -0.03 & 0.9777 \\
\hline MDate & 19383 & 1.0867 & 0.8880 & 155 & 1.22 & 0.2229 \\
\hline MDate & 19384 & 3.1193 & 0.7352 & 147 & 4.24 & $<.0001$ \\
\hline MDate & 19385 & 2.4533 & 1.0190 & 151 & 2.41 & 0.0173 \\
\hline MDate & 19386 & 1.2110 & 0.8880 & 155 & 1.36 & 0.1746 \\
\hline MDate & 19387 & 1.6260 & 1.0190 & 151 & 1.60 & 0.1127 \\
\hline MDate & 19388 & 0.7205 & 1.2392 & 114 & 0.58 & 0.5621 \\
\hline MDate & 19389 & 1.7083 & 0.8880 & 155 & 1.92 & 0.0562 \\
\hline MDate & 19390 & -1.1762 & 0.6859 & 145 & -1.71 & 0.0885 \\
\hline MDate & 19391 & 0.9678 & 1.2392 & 114 & 0.78 & 0.4364 \\
\hline MDate & 19392 & 0.3407 & 0.8880 & 155 & 0.38 & 0.7017 \\
\hline MDate & 19393 & -1.5177 & 1.0190 & 151 & -1.49 & 0.1385 \\
\hline MDate & 19394 & -1.8486 & 1.0190 & 151 & -1.81 & 0.0716 \\
\hline MDate & 19395 & 0.2259 & 1.2392 & 114 & 0.18 & 0.8557 \\
\hline MDate & 19396 & 0.7987 & 1.0190 & 151 & 0.78 & 0.4344 \\
\hline MDate & 19397 & 0.4732 & 1.2392 & 114 & 0.38 & 0.7033 \\
\hline MDate & 19398 & 0.8380 & 0.8880 & 155 & 0.94 & 0.3468 \\
\hline MDate & 19399 & 0.7205 & 1.2392 & 114 & 0.58 & 0.562 \\
\hline
\end{tabular}

The SAS System

09:55 Thursday, March 14, 20135

Analyte=Formate RefValue $=16$

The Mixed Procedure

Model Information

Data Set

WORK. TOMSORT 
SRNL-STI-2013-00139

Revision 0

\begin{tabular}{|c|c|c|c|c|}
\hline \multirow{2}{*}{\multicolumn{2}{|c|}{ Dependent Variable }} & \multirow{2}{*}{\multicolumn{3}{|c|}{ PctDiff }} \\
\hline & & & & \\
\hline \multicolumn{2}{|l|}{ Covariance Structure } & Variance & Compone & ents \\
\hline \multicolumn{2}{|l|}{ Estimation Method } & \multicolumn{3}{|c|}{ REML } \\
\hline \multicolumn{2}{|c|}{ Residual Variance Method } & \multicolumn{3}{|l|}{ Profile } \\
\hline \multicolumn{2}{|c|}{ Fixed Effects SE Method } & \multicolumn{3}{|c|}{$\begin{array}{l}\text { Prasad-Rao-Jeske- } \\
\text { Kackar-Harville }\end{array}$} \\
\hline Degrees of & Freedom Method & \multicolumn{3}{|c|}{ Kenward-Roger } \\
\hline \multicolumn{2}{|c|}{ Class Level } & \multicolumn{3}{|l|}{ Information } \\
\hline Levels & Values & & & \\
\hline \multirow[t]{17}{*}{ MDate } & 19306 & 1930719308 & 19310 & 19311 \\
\hline & 19313 & 1931419315 & 19316 & 19317 \\
\hline & 19318 & 1931919321 & 19322 & 19323 \\
\hline & 19324 & 1932519326 & 19327 & 19329 \\
\hline & 19330 & 1933119333 & 19335 & 19336 \\
\hline & 19337 & 1933819339 & 19341 & 19342 \\
\hline & 19343 & 1934419345 & 19347 & 19348 \\
\hline & 19349 & 1935019351 & 19352 & 19354 \\
\hline & 19355 & 1935619357 & 19358 & 19359 \\
\hline & 19360 & 1936119362 & 19363 & 19364 \\
\hline & 19365 & 1936619369 & 19370 & 19371 \\
\hline & 19374 & 1937519376 & 19377 & 19378 \\
\hline & 19379 & 1938019381 & 19382 & 19383 \\
\hline & 19384 & 1938519386 & 19387 & 19388 \\
\hline & 19389 & 1939019391 & 19392 & 19393 \\
\hline & 19394 & 1939519396 & 19397 & 19398 \\
\hline & 19399 & & & \\
\hline
\end{tabular}

Dimensions

$\begin{array}{lr}\text { Covariance Parameters } & 2 \\ \text { Columns in X } & 1 \\ \text { Columns in Z } & 81 \\ \text { Subjects } & 1 \\ \text { Max Obs Per Subject } & 158 \\ \text { Observations Used } & 158 \\ \text { Observations Not Used } & 0 \\ \text { Total Observations } & 158\end{array}$

The SAS System 09:55 Thursday, March 14, 20136

Analyte=Formate RefValue $=16$

The Mixed Procedure

Iteration History

$\begin{array}{rrrr}\text { Iteration } & \text { Evaluations } & -2 \text { Res Log Like } & \text { Criterion } \\ 0 & 1 & 593.54995695 & \\ 1 & 2 & 592.26684550 & 0.00000098 \\ 2 & 1 & 592.26669674 & 0.00000000\end{array}$


SRNL-STI-2013-00139

Revision 0

Convergence criteria met.

Covariance Parameter Estimates

$\begin{array}{lrrrr}\text { Cov Parm } & \text { Estimate } & \text { Alpha } & \text { Lower } & \text { Upper } \\ & & & & \\ \text { MDate } & 0.2923 & 0.05 & -0.2725 & 0.8571 \\ \text { Residual } & 2.2000 & 0.05 & 1.6550 & 3.0680\end{array}$

Asymptotic Covariance Matrix of Estimates

$\begin{array}{rlrr}\text { Row } & \text { Cov Parm } & \text { CovP1 } & \text { CovP2 } \\ 1 & \text { MDate } & 0.08304 & -0.06010 \\ 2 & \text { Residual } & -0.06010 & 0.1186\end{array}$

Fit Statistics

$\begin{array}{ll}\text {-2 Res Log Likelihood } & 592.3 \\ \text { AIC (smaller is better) } & 596.3 \\ \text { AICC (smaller is better) } & 596.3 \\ \text { BIC (smaller is better) } & 601.1\end{array}$

Null Model Likelihood Ratio Test

DF Chi-Square $\quad \mathrm{Pr}>\mathrm{ChiSq}$

$\begin{array}{lll}1 & 1.28 & 0.2573\end{array}$

The SAS System 09:55 Thursday, March 14, 20137

Analyte=Formate RefValue=16

The Mixed Procedure

Solution for Fixed Effects

$\begin{array}{lrrrrr}\text { Effect } & \text { Estimate } & \begin{array}{r}\text { Standard } \\ \text { Error }\end{array} & \text { DF } & \text { t value } & \operatorname{Pr}>|t| \\ \text { Intercept } & 1.6713 & 0.1370 & 59.7 & 12.20 & <.0001\end{array}$

Solution for Random Effects

$\begin{array}{lrrrrrr}\text { Effect } & \text { MDate } & \text { Estimate } & \begin{array}{r}\text { Std Err } \\ \text { Pred }\end{array} & \text { DF } & \text { t Value } & \text { Pr }>|t| \\ & & & & & & \\ \text { MDate } & 19306 & 0.1485 & 0.5665 & 2.7 & 0.26 & 0.8119 \\ \text { MDate } & 19307 & -0.1300 & 0.5665 & 2.7 & -0.23 & 0.8347\end{array}$


SRNL-STI-2013-00139

Revision 0

$\begin{array}{lrrrrrr}\text { MDate } & 19308 & -0.1813 & 0.5665 & 2.7 & -0.32 & 0.7720 \\ \text { MDate } & 19310 & -0.2000 & 0.5667 & 3.43 & -0.35 & 0.7448 \\ \text { MDate } & 19311 & -0.2400 & 0.5665 & 2.7 & -0.42 & 0.7033 \\ \text { MDate } & 19313 & 0.02308 & 0.5667 & 3.43 & 0.04 & 0.9698 \\ \text { MDate } & 19314 & 0.08253 & 0.5665 & 2.7 & 0.15 & 0.8944 \\ \text { MDate } & 19315 & -0.1080 & 0.5665 & 2.7 & -0.19 & 0.8622 \\ \text { MDate } & 19316 & -0.01276 & 0.5665 & 2.7 & -0.02 & 0.9836 \\ \text { MDate } & 19317 & -0.03475 & 0.5665 & 2.7 & -0.06 & 0.9553 \\ \text { MDate } & 19318 & 0.05589 & 0.5667 & 3.43 & 0.10 & 0.9269 \\ \text { MDate } & 19319 & -0.4599 & 0.5665 & 2.7 & -0.81 & 0.4823 \\ \text { MDate } & 19321 & -0.2546 & 0.5422 & 5.15 & -0.47 & 0.6579 \\ \text { MDate } & 19322 & -0.2546 & 0.5422 & 5.15 & -0.47 & 0.6579 \\ \text { MDate } & 19323 & -0.4164 & 0.5667 & 3.43 & -0.73 & 0.5094 \\ \text { MDate } & 19324 & 0.2364 & 0.5665 & 2.7 & 0.42 & 0.7073 \\ \text { MDate } & 19325 & 0.5924 & 0.5566 & 4.25 & 1.06 & 0.3439 \\ \text { MDate } & 19326 & 0.2218 & 0.5665 & 2.7 & 0.39 & 0.7242 \\ \text { MDate } & 19327 & -0.2986 & 0.5665 & 2.7 & -0.53 & 0.6383 \\ \text { MDate } & 19329 & -0.08188 & 0.5667 & 3.43 & -0.14 & 0.8932 \\ \text { MDate } & 19330 & -0.1016 & 0.5667 & 3.43 & -0.18 & 0.8678 \\ \text { MDate } & 19331 & 0.1215 & 0.5667 & 3.43 & 0.21 & 0.8424 \\ \text { MDate } & 19333 & 0.4298 & 0.5667 & 3.43 & 0.76 & 0.4969 \\ \text { MDate } & 19335 & -0.1154 & 0.5665 & 2.7 & -0.20 & 0.8530 \\ \text { MDate } & 19336 & 0.3133 & 0.5566 & 4.25 & 0.56 & 0.6018 \\ \text { MDate } & 19337 & 0.1925 & 0.5665 & 2.7 & 0.34 & 0.7587 \\ \text { MDate } & 19338 & -0.2693 & 0.5665 & 2.7 & -0.48 & 0.6703 \\ \text { MDate } & 19339 & -0.3792 & 0.5665 & 2.7 & -0.67 & 0.5559 \\ \text { MDate } & 19341 & 0.2002 & 0.5667 & 3.43 & 0.35 & 0.7445 \\ \text { MDate } & 19342 & -0.06069 & 0.5566 & 4.25 & -0.11 & 0.9181 \\ \text { MDate } & 19343 & 0.05321 & 0.5665 & 2.7 & 0.09 & 0.9317 \\ \text { MDate } & 19344 & 0.1293 & 0.5566 & 4.25 & 0.23 & 0.8271 \\ \text { MDate } & 19345 & -0.02009 & 0.5665 & 2.7 & -0.04 & 0.9742 \\ \text { MDate } & 19347 & -0.3115 & 0.5667 & 3.43 & -0.55 & 0.6163 \\ \text { MDate } & 19348 & 0.07557 & 0.5667 & 3.43 & 0.13 & 0.9014 \\ \text { MDate } & 19349 & -0.02009 & 0.5665 & 2.7 & -0.04 & 0.9742\end{array}$

The SAS System

09:55 Thursday, March 14, 20138

Analyte $=$ Formate RefValue $=16$

The Mixed Procedure

Solution for Random Effects

$\begin{array}{lrrrrrr}\text { Effect } & \text { MDate } & \text { Estimate } & \begin{array}{r}\text { Std Err } \\ \text { Pred }\end{array} & \text { DF } & t \text { Value } & \text { Pr }>|t| \\ & & & & & & \\ \text { MDate } & 19350 & -0.2980 & 0.5422 & 5.15 & -0.55 & 0.6056 \\ \text { MDate } & 19351 & 0.1543 & 0.5667 & 3.43 & 0.27 & 0.8010 \\ \text { MDate } & 19352 & -0.1147 & 0.5667 & 3.43 & -0.20 & 0.8510 \\ \text { MDate } & 19354 & 0.2955 & 0.5566 & 4.25 & 0.53 & 0.6220 \\ \text { MDate } & 19355 & 0.03431 & 0.5566 & 4.25 & 0.06 & 0.9536 \\ \text { MDate } & 19356 & 0.07586 & 0.5566 & 4.25 & 0.14 & 0.8978 \\ \text { MDate } & 19357 & 0.1118 & 0.5665 & 2.7 & 0.20 & 0.8574 \\ \text { MDate } & 19358 & 0.1805 & 0.5667 & 3.43 & 0.32 & 0.7685 \\ \text { MDate } & 19359 & 0.1543 & 0.5667 & 3.43 & 0.27 & 0.8010 \\ \text { MDate } & 19360 & 0.06054 & 0.5665 & 2.7 & 0.11 & 0.9223 \\ \text { MDate } & 19361 & -0.1212 & 0.5667 & 3.43 & -0.21 & 0.8427\end{array}$


SRNL-STI-2013-00139

Revision 0

$\begin{array}{lrrrrrr}\text { MDate } & 19362 & -0.1813 & 0.5665 & 2.7 & -0.32 & 0.7720 \\ \text { MDate } & 19363 & -0.1737 & 0.5667 & 3.43 & -0.31 & 0.7769 \\ \text { MDate } & 19364 & -0.3646 & 0.5665 & 2.7 & -0.64 & 0.5703 \\ \text { MDate } & 19365 & -0.4379 & 0.5665 & 2.7 & -0.77 & 0.5015 \\ \text { MDate } & 19366 & -0.2546 & 0.5665 & 2.7 & -0.45 & 0.6867 \\ \text { MDate } & 19369 & 0.1192 & 0.5665 & 2.7 & 0.21 & 0.8482 \\ \text { MDate } & 19370 & -0.5258 & 0.5665 & 2.7 & -0.93 & 0.4285 \\ \text { MDate } & 19371 & -0.1447 & 0.5665 & 2.7 & -0.26 & 0.8166 \\ \text { MDate } & 19374 & -0.04941 & 0.5665 & 2.7 & -0.09 & 0.9366 \\ \text { MDate } & 19375 & -0.01276 & 0.5665 & 2.7 & -0.02 & 0.9836 \\ \text { MDate } & 19376 & -0.5224 & 0.5265 & 6.13 & -0.99 & 0.3587 \\ \text { MDate } & 19377 & 0.3773 & 0.5667 & 3.43 & 0.67 & 0.5476 \\ \text { MDate } & 19378 & 0.1674 & 0.5667 & 3.43 & 0.30 & 0.7847 \\ \text { MDate } & 19379 & -0.1497 & 0.5566 & 4.25 & -0.27 & 0.8005 \\ \text { MDate } & 19380 & 0.05212 & 0.5566 & 4.25 & 0.09 & 0.9296 \\ \text { MDate } & 19381 & -0.04317 & 0.5422 & 5.15 & -0.08 & 0.9395 \\ \text { MDate } & 19382 & 0.3249 & 0.5667 & 3.43 & 0.57 & 0.6019 \\ \text { MDate } & 19383 & 0.4618 & 0.5566 & 4.25 & 0.83 & 0.4508 \\ \text { MDate } & 19384 & 0.4655 & 0.5265 & 6.13 & 0.88 & 0.4100 \\ \text { MDate } & 19385 & 0.3380 & 0.5667 & 3.43 & 0.60 & 0.5880 \\ \text { MDate } & 19386 & -0.00725 & 0.5566 & 4.25 & -0.01 & 0.9902 \\ \text { MDate } & 19387 & 0.2133 & 0.5667 & 3.43 & 0.38 & 0.7287 \\ \text { MDate } & 19388 & 0.03855 & 0.5665 & 2.7 & 0.07 & 0.9505 \\ \text { MDate } & 19389 & -0.00725 & 0.5566 & 4.25 & -0.01 & 0.9902 \\ \text { MDate } & 19390 & -0.04825 & 0.5106 & 7.18 & -0.09 & 0.9273 \\ \text { MDate } & 19391 & 0.1632 & 0.5665 & 2.7 & 0.29 & 0.7940 \\ \text { MDate } & 19392 & 0.3905 & 0.5566 & 4.25 & 0.70 & 0.5194 \\ \text { MDate } & 19393 & -0.1737 & 0.5667 & 3.43 & -0.31 & 0.7769 \\ \text { MDate } & 19394 & 0.1265 & 0.5665 & 2.7 & 0.22 & 0.8391 \\ \text { MDate } & 19395 & -0.06406 & 0.5665 & 2.7 & -0.11 & 0.9178 \\ \text { MDate } & 19396 & -0.02284 & 0.5667 & 3.43 & -0.04 & 0.9701 \\ \text { MDate } & 19397 & 0.1045 & 0.5665 & 2.7 & 0.18 & 0.8666 \\ \text { MDate } & 19398 & 0.2777 & 0.5566 & 4.25 & 0.50 & 0.6425\end{array}$

The SAS System

09:55 Thursday, March 14, 20139

Analyte=Formate RefValue $=16$

The Mixed Procedure

Solution for Random Effects

\begin{tabular}{|c|c|c|c|c|c|c|}
\hline & & & Std Err & & & \\
\hline Effect & MDate & Estimate & Pred & DF & t Value & $\mathrm{Pr}>|\mathrm{t}|$ \\
\hline Date & 19399 & 0.1045 & 0.5665 & 2.7 & 0.18 & 0.8666 \\
\hline
\end{tabular}

The SAS System 09:55 Thursday, March 14, 201310

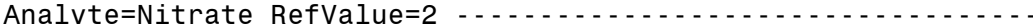

The Mixed Procedure

Model Information

Data Set

Dependent Variable

WORK. TOMSORT

PctDiff 
SRNL-STI-2013-00139

Revision 0

$\begin{array}{ll}\text { Covariance Structure } & \text { Variance Components } \\ \text { Estimation Method } & \text { REML } \\ \text { Residual Variance Method } & \text { Profile } \\ \text { Fixed Effects SE Method } & \text { Prasad-Rao-Jeske- } \\ & \begin{array}{l}\text { Kackar-Harville } \\ \text { Degrees of Freedom Method }\end{array} \\ \end{array}$

Class Level Information

\begin{tabular}{lllllll} 
Class Levels & \multicolumn{1}{l}{ Values } \\
MDate & 83 & 19306 & 19307 & 19308 & 19310 & 19311 \\
& 19313 & 19314 & 19315 & 19316 & 19317 \\
19318 & 19319 & 19321 & 19322 & 19323 \\
& 19324 & 19325 & 19326 & 19327 & 19329 \\
19330 & 19331 & 19333 & 19334 & 19335 \\
19336 & 19337 & 19338 & 19339 & 19341 \\
19342 & 19343 & 19344 & 19345 & 19347 \\
19348 & 19349 & 19350 & 19351 & 19352 \\
19354 & 19355 & 19356 & 19357 & 19358 \\
19359 & 19360 & 19361 & 19362 & 19363 \\
19364 & 19366 & 19369 & 19370 & 19371 \\
19372 & 19373 & 19374 & 19375 & 19376 \\
19377 & 19378 & 19379 & 19380 & 19381 \\
& 19382 & 19383 & 19384 & 19385 & 19386 \\
19387 & 19388 & 19389 & 19390 & 19391 \\
19392 & 19393 & 19394 & 19395 & 19396 \\
& 19397 & 19398 & 19399 & &
\end{tabular}

Dimensions

$\begin{array}{lr}\text { Covariance Parameters } & 2 \\ \text { Columns in X } & 1 \\ \text { Columns in Z } & 83 \\ \text { Subjects } & 1 \\ \text { Max Obs Per Subject } & 167 \\ \text { Observations Used } & 167 \\ \text { Observations Not Used } & 0 \\ \text { Total Observations } & 167\end{array}$

The SAS System

09:55 Thursday, March 14, 201311

Analyte $=$ Nitrate RefValue $=2$

The Mixed Procedure

Iteration History

$\begin{array}{rrrr}\text { Iteration } & \text { Evaluations } & -2 \text { Res Log Like } & \text { Criterion } \\ 0 & 1 & 843.91579313 & \\ 1 & 2 & 786.10905211 & 0.00326664 \\ 2 & 1 & 785.20726673 & 0.00023715 \\ 3 & 1 & 785.14749809 & 0.00000150 \\ 4 & 1 & 785.14713633 & 0.00000000\end{array}$


SRNL-STI-2013-00139

Revision 0

Convergence criteria met.

Covariance Parameter Estimates

$\begin{array}{lrrrr}\text { Cov Parm } & \text { Estimate } & \text { Alpha } & \text { Lower } & \text { Upper } \\ & & & & \\ \text { MDate } & 5.9456 & 0.05 & 3.5138 & 8.3773 \\ \text { Residual } & 3.0773 & 0.05 & 2.3308 & 4.2522\end{array}$

Asymptotic Covariance Matrix of Estimates

$\begin{array}{rlrr}\text { Row } & \text { Cov Parm } & \text { CovP1 } & \text { CovP2 } \\ 1 & \text { MDate } & 1.5394 & -0.1234 \\ 2 & \text { Residual } & -0.1234 & 0.2203\end{array}$

Fit Statistics

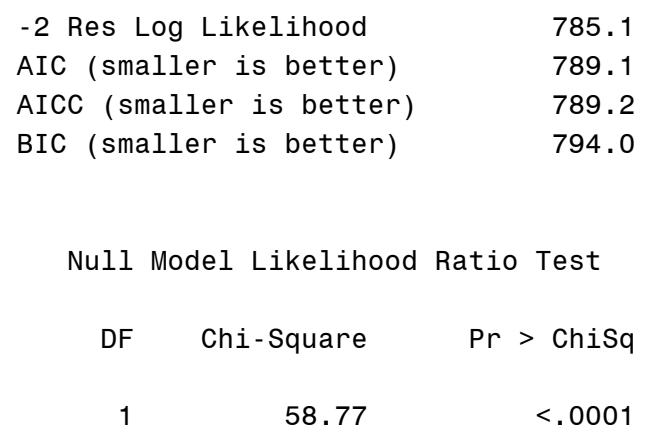

The SAS System

09:55 Thursday, March 14, 2013

Analyte $=$ Nitrate RefValue $=2$

The Mixed Procedure

Solution for Fixed Effects

$\begin{array}{lrrrrr}\text { Effect } & \text { Estimate } & \begin{array}{r}\text { Standard } \\ \text { Error }\end{array} & \text { DF } & t \text { Value } & \mathrm{Pr}>|\mathrm{t}| \\ \text { Intercept } & -0.4384 & 0.3079 & 81.6 & -1.42 & 0.1583\end{array}$

Solution for Random Effects

$\begin{array}{lrrrrrr}\text { Effect } & \text { MDate } & \text { Estimate } & \begin{array}{r}\text { Std Err } \\ \text { Pred }\end{array} & \text { DF } & \text { t Value } & \text { Pr }>|t| \\ \text { MDate } & 19306 & 0.9478 & 1.4632 & 166 & 0.65 & 0.5180\end{array}$


SRNL-STI-2013-00139

Revision 0

$\begin{array}{lrrrrrr}\text { MDate } & 19307 & -0.04061 & 1.4632 & 166 & -0.03 & 0.9779 \\ \text { MDate } & 19308 & -0.3701 & 1.4632 & 166 & -0.25 & 0.8006 \\ \text { MDate } & 19310 & -1.4392 & 1.1463 & 151 & -1.26 & 0.2112 \\ \text { MDate } & 19311 & -1.3585 & 1.4632 & 166 & -0.93 & 0.3545 \\ \text { MDate } & 19313 & -0.6448 & 1.1463 & 151 & -0.56 & 0.5746 \\ \text { MDate } & 19314 & -2.0174 & 1.4632 & 166 & -1.38 & 0.1698 \\ \text { MDate } & 19315 & -1.0290 & 1.4632 & 166 & -0.70 & 0.4829 \\ \text { MDate } & 19316 & -0.6996 & 1.4632 & 166 & -0.48 & 0.6332 \\ \text { MDate } & 19317 & -1.0290 & 1.4632 & 166 & -0.70 & 0.4829 \\ \text { MDate } & 19318 & -1.6161 & 0.9805 & 142 & -1.65 & 0.1015 \\ \text { MDate } & 19319 & -3.0059 & 1.4632 & 166 & -2.05 & 0.0415 \\ \text { MDate } & 19321 & -1.7148 & 0.8757 & 139 & -1.96 & 0.0522 \\ \text { MDate } & 19322 & 1.3843 & 0.8757 & 139 & 1.58 & 0.1162 \\ \text { MDate } & 19323 & 3.9231 & 1.1463 & 151 & 3.42 & 0.0008 \\ \text { MDate } & 19324 & 3.9131 & 1.4632 & 166 & 2.67 & 0.0082 \\ \text { MDate } & 19325 & 4.4960 & 0.9805 & 142 & 4.59 & <.0001 \\ \text { MDate } & 19326 & 3.5836 & 1.4632 & 166 & 2.45 & 0.0154 \\ \text { MDate } & 19327 & 0.9478 & 1.4632 & 166 & 0.65 & 0.5180 \\ \text { MDate } & 19329 & -0.4462 & 1.1463 & 151 & -0.39 & 0.6976 \\ \text { MDate } & 19330 & -0.04896 & 1.1463 & 151 & -0.04 & 0.9660 \\ \text { MDate } & 19331 & -3.9943 & 1.4632 & 166 & -2.73 & 0.0070 \\ \text { MDate } & 19333 & -0.04896 & 1.1463 & 151 & -0.04 & 0.9660 \\ \text { MDate } & 19334 & -3.3353 & 1.4632 & 166 & -2.28 & 0.0239 \\ \text { MDate } & 19335 & -0.6996 & 1.4632 & 166 & -0.48 & 0.6332 \\ \text { MDate } & 19336 & -1.3827 & 0.8757 & 139 & -1.58 & 0.1166 \\ \text { MDate } & 19337 & -0.6996 & 1.4632 & 166 & -0.48 & 0.6332 \\ \text { MDate } & 19338 & -2.6764 & 1.4632 & 166 & -1.83 & 0.0692 \\ \text { MDate } & 19339 & -3.6238 & 1.1463 & 151 & -3.16 & 0.0019 \\ \text { MDate } & 19341 & -0.4462 & 1.1463 & 151 & -0.39 & 0.6976 \\ \text { MDate } & 19342 & -1.7583 & 0.9805 & 142 & -1.79 & 0.0751 \\ \text { MDate } & 19343 & -1.0290 & 1.4632 & 166 & -0.70 & 0.4829 \\ \text { MDate } & 19344 & -1.2406 & 1.1463 & 151 & -1.08 & 0.2809 \\ \text { MDate } & 19345 & -1.3585 & 1.4632 & 166 & -0.93 & 0.3545 \\ \text { MDate } & 19347 & -0.4462 & 1.1463 & 151 & -0.39 & 0.6976 \\ \text { MDate } & 19348 & -1.4392 & 1.1463 & 151 & -1.26 & 0.2112\end{array}$

The SAS System

09:55 Thursday, March 14, 201313

Analyte $=$ Nitrate RefValue $=2$

The Mixed Procedure

Solution for Random Effects

$\begin{array}{lcrrrrr}\text { Effect } & \text { MDate } & \text { Estimate } & \begin{array}{c}\text { Std Err } \\ \text { Pred }\end{array} & \text { DF } & \text { t Value } & \text { Pr }>|t| \\ & & & & & & \\ \text { MDate } & 19349 & -0.6996 & 1.4632 & 166 & -0.48 & 0.6332 \\ \text { MDate } & 19350 & 0.2775 & 0.8757 & 139 & 0.32 & 0.7518 \\ \text { MDate } & 19351 & -1.6378 & 1.1463 & 151 & -1.43 & 0.1551 \\ \text { MDate } & 19352 & -2.0350 & 1.1463 & 151 & -1.78 & 0.0779 \\ \text { MDate } & 19354 & -0.4790 & 0.9805 & 142 & -0.49 & 0.6260 \\ \text { MDate } & 19355 & -2.4690 & 0.9805 & 142 & -2.52 & 0.0129 \\ \text { MDate } & 19356 & -0.9054 & 0.9805 & 142 & -0.92 & 0.3574 \\ \text { MDate } & 19357 & -0.3701 & 1.4632 & 166 & -0.25 & 0.8006 \\ \text { MDate } & 19358 & -1.2406 & 1.1463 & 151 & -1.08 & 0.2809 \\ \text { MDate } & 19359 & -0.6448 & 1.1463 & 151 & -0.56 & 0.5746\end{array}$


SRNL-STI-2013-00139

Revision 0

$\begin{array}{lrrrrrr}\text { MDate } & 19360 & -0.6996 & 1.4632 & 166 & -0.48 & 0.6332 \\ \text { MDate } & 19361 & -2.0350 & 1.1463 & 151 & -1.78 & 0.0779 \\ \text { MDate } & 19362 & -2.3469 & 1.4632 & 166 & -1.60 & 0.1106 \\ \text { MDate } & 19363 & -1.0420 & 1.1463 & 151 & -0.91 & 0.3648 \\ \text { MDate } & 19364 & -2.3469 & 1.4632 & 166 & -1.60 & 0.1106 \\ \text { MDate } & 19366 & -3.0059 & 1.4632 & 166 & -2.05 & 0.0415 \\ \text { MDate } & 19369 & -1.0290 & 1.4632 & 166 & -0.70 & 0.4829 \\ \text { MDate } & 19370 & -3.0059 & 1.4632 & 166 & -2.05 & 0.0415 \\ \text { MDate } & 19371 & -2.8954 & 0.9805 & 142 & -2.95 & 0.0037 \\ \text { MDate } & 19372 & -4.1337 & 0.8021 & 138 & -5.15 & <.0001 \\ \text { MDate } & 19373 & 0.6183 & 1.4632 & 166 & 0.42 & 0.6731 \\ \text { MDate } & 19374 & -0.04061 & 1.4632 & 166 & -0.03 & 0.9779 \\ \text { MDate } & 19375 & -0.04061 & 1.4632 & 166 & -0.03 & 0.9779 \\ \text { MDate } & 19376 & 2.9346 & 0.8021 & 138 & 3.66 & 0.0004 \\ \text { MDate } & 19377 & 2.9324 & 0.9805 & 142 & 2.99 & 0.0033 \\ \text { MDate } & 19378 & 1.9362 & 1.4632 & 166 & 1.32 & 0.1876 \\ \text { MDate } & 19379 & 3.2167 & 0.9805 & 142 & 3.28 & 0.0013 \\ \text { MDate } & 19380 & 2.3639 & 0.9805 & 142 & 2.41 & 0.0172 \\ \text { MDate } & 19381 & 2.0483 & 0.8757 & 139 & 2.34 & 0.0208 \\ \text { MDate } & 19382 & 3.7245 & 1.1463 & 151 & 3.25 & 0.0014 \\ \text { MDate } & 19383 & 2.9324 & 0.9805 & 142 & 2.99 & 0.0033 \\ \text { MDate } & 19384 & 3.7502 & 0.8021 & 138 & 4.68 & <.0001 \\ \text { MDate } & 19385 & 3.1287 & 1.1463 & 151 & 2.73 & 0.0071 \\ \text { MDate } & 19386 & 1.7953 & 0.9805 & 142 & 1.83 & 0.0692 \\ \text { MDate } & 19387 & 2.5329 & 1.1463 & 151 & 2.21 & 0.0286 \\ \text { MDate } & 19388 & 1.9362 & 1.4632 & 166 & 1.32 & 0.1876 \\ \text { MDate } & 19389 & 2.7315 & 1.1463 & 151 & 2.38 & 0.0184 \\ \text { MDate } & 19390 & 1.0173 & 0.7472 & 139 & 1.36 & 0.1756 \\ \text { MDate } & 19391 & 2.5952 & 1.4632 & 166 & 1.77 & 0.0780 \\ \text { MDate } & 19392 & 1.6532 & 0.9805 & 142 & 1.69 & 0.0940 \\ \text { MDate } & 19393 & 0.1496 & 1.1463 & 151 & 0.13 & 0.8963 \\ \text { MDate } & 19394 & 0.1496 & 1.1463 & 151 & 0.13 & 0.8963 \\ \text { MDate } & 19395 & 0.9478 & 1.4632 & 166 & 0.65 & 0.5180 \\ \text { MDate } & 19396 & 1.9371 & 1.1463 & 151 & 1.69 & 0.0931\end{array}$

The SAS System

09:55 Thursday, March 14, 201314

Analyte $=$ Nitrate RefValue $=2$

The Mixed Procedure

Solution for Random Effects

$\begin{array}{lrrrrrr}\text { Effect } & \text { MDate } & \text { Estimate } & \begin{array}{r}\text { Std Err } \\ \text { Pred }\end{array} & \text { DF } & \text { t Value } & \text { Pr }>|t| \\ \text { MDate } & 19397 & 1.6067 & 1.4632 & 166 & 1.10 & 0.2737 \\ \text { MDate } & 19398 & 2.3639 & 0.9805 & 142 & 2.41 & 0.0172 \\ \text { MDate } & 19399 & 2.2657 & 1.4632 & 166 & 1.55 & 0.1234\end{array}$

The SAS System

09:55 Thursday, March 14, 201315

Analyte $=$ Nitrate RefValue $=16$

The Mixed Procedure

Model Information 
SRNL-STI-2013-00139

Revision 0

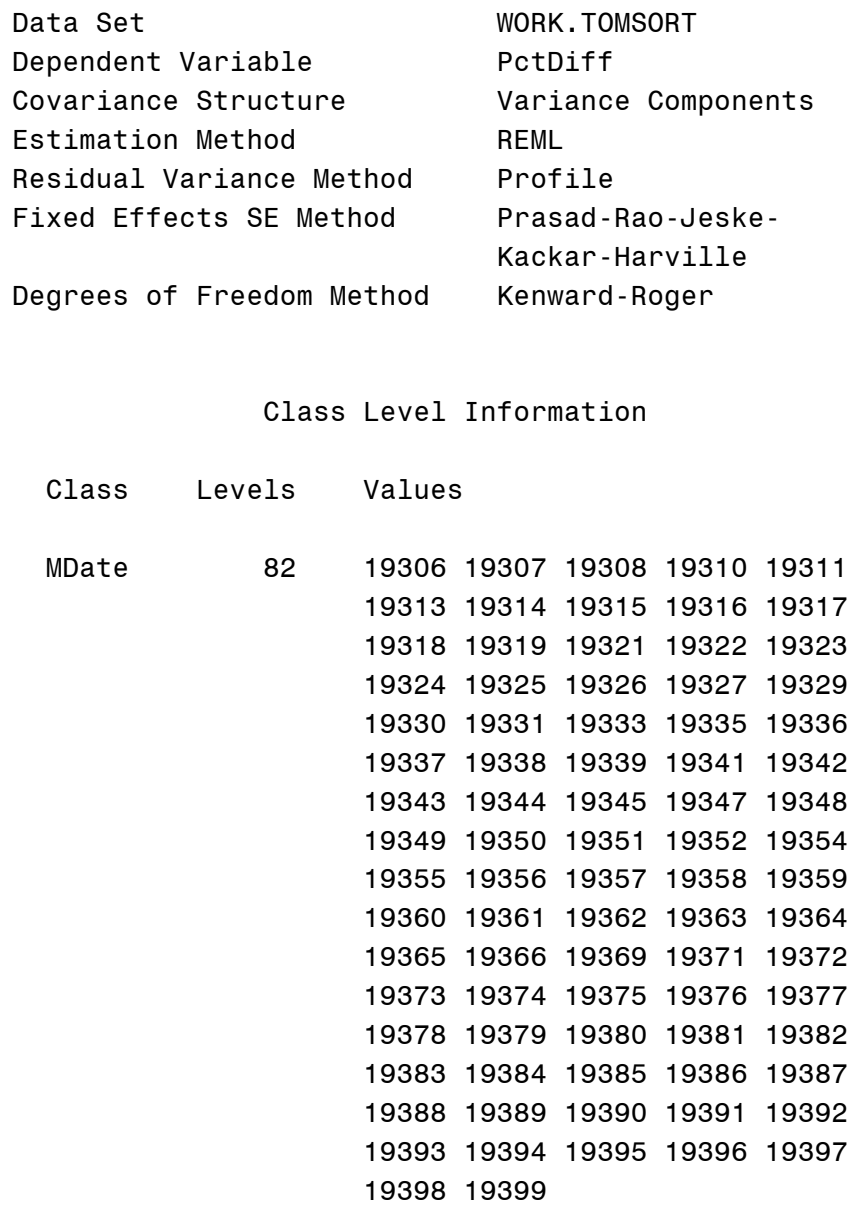

Dimensions

$\begin{array}{lr}\text { Covariance Parameters } & 2 \\ \text { Columns in X } & 1 \\ \text { Columns in Z } & 82 \\ \text { Subjects } & 1 \\ \text { Max Obs Per Subject } & 157 \\ \text { Observations Used } & 157 \\ \text { Observations Not Used } & 0 \\ \text { Total Observations } & 157\end{array}$

The SAS System 09:55 Thursday, March 14, 201316

Analyte=Nitrate RefValue=16 $\ldots \ldots$

The Mixed Procedure

Iteration History

$\begin{array}{rrrr}\text { Iteration } & \text { Evaluations } & -2 \text { Res Log Like } & \text { Criterion } \\ 0 & 1 & 581.52544054 & \\ 1 & 2 & 576.87668549 & 0.00103196\end{array}$


SRNL-STI-2013-00139

Revision 0

Convergence criteria met.

Covariance Parameter Estimates

$\begin{array}{lrrrr}\text { Cov Parm } & \text { Estimate } & \text { Alpha } & \text { Lower } & \text { Upper } \\ & & & & \\ \text { MDate } & 0.7298 & 0.05 & 0.01932 & 1.4404 \\ \text { Residual } & 1.7000 & 0.05 & 1.2376 & 2.4816\end{array}$

Asymptotic Covariance Matrix of Estimates

$\begin{array}{rlrr}\text { Row } & \text { Cov Parm } & \text { CovP1 } & \text { CovP2 } \\ & & & \\ 1 & \text { MDate } & 0.1314 & -0.06505 \\ 2 & \text { Residual } & -0.06505 & 0.08965\end{array}$

Fit Statistics

$\begin{array}{ll}\text {-2 Res Log Likelihood } & 576.7 \\ \text { AIC (smaller is better) } & 580.7 \\ \text { AICC (smaller is better) } & 580.8 \\ \text { BIC (smaller is better) } & 585.5\end{array}$

Null Model Likelihood Ratio Test

DF Chi-Square $\quad \mathrm{Pr}>$ ChiSq

$1 \quad 4.82 \quad 0.0282$

The SAS System

09:55 Thursday, March 14, 201317

Analyte=Nitrate RefValue=16 $\ldots$

The Mixed Procedure

Solution for Fixed Effects

$\begin{array}{lrrrrr}\text { Effect } & \text { Estimate } & \begin{array}{r}\text { Standard } \\ \text { Error }\end{array} & \text { DF } & t \text { Value } & \operatorname{Pr}>|t| \\ \text { Intercept } & 0.4697 & 0.1464 & 57.7 & 3.21 & 0.0022\end{array}$

Solution for Random Effects

Std Err 
SRNL-STI-2013-00139

Revision 0

\begin{tabular}{|c|c|c|c|c|c|c|}
\hline Effect & MDate & Estimate & Pred & DF & t Value & $\operatorname{Pr}>|t|$ \\
\hline MDate & 19306 & 0.7976 & 0.7705 & 19.4 & 1.04 & 0.3133 \\
\hline MDate & 19307 & 0.2532 & 0.7705 & 19.4 & 0.33 & 0.7460 \\
\hline MDate & 19308 & 0.08420 & 0.7705 & 19.4 & 0.11 & 0.9141 \\
\hline MDate & 19310 & -0.1592 & 0.6863 & 36.5 & -0.23 & 0.8178 \\
\hline MDate & 19311 & -0.2349 & 0.7705 & 19.4 & -0.30 & 0.7637 \\
\hline MDate & 19313 & 0.6637 & 0.6863 & 36.5 & 0.97 & 0.3399 \\
\hline MDate & 19314 & 0.5347 & 0.7705 & 19.4 & 0.69 & 0.4959 \\
\hline MDate & 19315 & 0.1968 & 0.7705 & 19.4 & 0.26 & 0.8010 \\
\hline MDate & 19316 & 0.3095 & 0.7705 & 19.4 & 0.40 & 0.6923 \\
\hline MDate & 19317 & -0.06598 & 0.7705 & 19.4 & -0.09 & 0.9326 \\
\hline MDate & 19318 & 0.3605 & 0.6863 & 36.5 & 0.53 & 0.6026 \\
\hline MDate & 19319 & -1.0985 & 0.7705 & 19.4 & -1.43 & 0.1698 \\
\hline MDate & 19321 & -0.05983 & 0.5700 & 81.5 & -0.10 & 0.9167 \\
\hline MDate & 19322 & 0.06854 & 0.5700 & 81.5 & 0.12 & 0.9046 \\
\hline MDate & 19323 & -0.7656 & 0.6863 & 36.5 & -1.12 & 0.2719 \\
\hline MDate & 19324 & 0.5160 & 0.7705 & 19.4 & 0.67 & 0.5110 \\
\hline MDate & 19325 & 0.9787 & 0.6208 & 58.2 & 1.58 & 0.1203 \\
\hline MDate & 19326 & 0.5723 & 0.7705 & 19.4 & 0.74 & 0.4665 \\
\hline MDate & 19327 & -0.7043 & 0.7705 & 19.4 & -0.91 & 0.3719 \\
\hline MDate & 19329 & -0.2025 & 0.6863 & 36.5 & -0.30 & 0.7696 \\
\hline MDate & 19330 & -0.3902 & 0.6863 & 36.5 & -0.57 & 0.5731 \\
\hline MDate & 19331 & -0.2537 & 0.7705 & 19.4 & -0.33 & 0.7455 \\
\hline MDate & 19333 & 0.4760 & 0.6863 & 36.5 & 0.69 & 0.4923 \\
\hline MDate & 19335 & -0.5353 & 0.7705 & 19.4 & -0.69 & 0.4954 \\
\hline MDate & 19336 & -0.2376 & 0.5700 & 81.5 & -0.42 & 0.6779 \\
\hline MDate & 19337 & 0.3095 & 0.7705 & 19.4 & 0.40 & 0.6923 \\
\hline MDate & 19338 & -0.7043 & 0.7705 & 19.4 & -0.91 & 0.3719 \\
\hline MDate & 19339 & -1.1360 & 0.7705 & 19.4 & -1.47 & 0.1564 \\
\hline MDate & 19341 & 0.6637 & 0.6863 & 36.5 & 0.97 & 0.3399 \\
\hline MDate & 19342 & 0.005344 & 0.6208 & 58.2 & 0.01 & 0.9932 \\
\hline MDate & 19343 & 0.04665 & 0.7705 & 19.4 & 0.06 & 0.9523 \\
\hline MDate & 19344 & 0.04053 & 0.6208 & 58.2 & 0.07 & 0.9482 \\
\hline MDate & 19345 & 0.06543 & 0.7705 & 19.4 & 0.08 & 0.9332 \\
\hline MDate & 19347 & -0.4913 & 0.6863 & 36.5 & -0.72 & 0.4786 \\
\hline MDate & 19348 & 0.4182 & 0.6863 & 36.5 & 0.61 & 0.5460 \\
\hline MDate & 19349 & 0.2907 & 0.7705 & 19.4 & 0.38 & 0.7100 \\
\hline
\end{tabular}

The SAS System

09:55 Thursday, March 14, 201318

Analyte $=$ Nitrate RefValue $=16$

The Mixed Procedure

Solution for Random Effects

$\begin{array}{lrrrrrr}\text { Effect } & \text { MDate } & \text { Estimate } & \begin{array}{r}\text { Std Err } \\ \text { Pred }\end{array} & \text { DF } & \text { t Value } & \text { Pr }>|t| \\ & & & & & & \\ \text { MDate } & 19350 & 0.02904 & 0.5700 & 81.5 & 0.05 & 0.9595 \\ \text { MDate } & 19351 & 0.3894 & 0.6863 & 36.5 & 0.57 & 0.5739 \\ \text { MDate } & 19352 & -0.4191 & 0.6863 & 36.5 & -0.61 & 0.5452 \\ \text { MDate } & 19354 & 0.7911 & 0.6208 & 58.2 & 1.27 & 0.2076 \\ \text { MDate } & 19355 & 0.1813 & 0.6208 & 58.2 & 0.29 & 0.7713 \\ \text { MDate } & 19356 & 0.2282 & 0.6208 & 58.2 & 0.37 & 0.7145 \\ \text { MDate } & 19357 & 0.4033 & 0.7705 & 19.4 & 0.52 & 0.6066\end{array}$


SRNL-STI-2013-00139

Revision 0

\begin{tabular}{|c|c|c|c|c|c|c|}
\hline MDate & 19358 & 0.5337 & 0.6863 & 36.5 & 0.78 & 0.4417 \\
\hline MDate & 19359 & 0.3749 & 0.6863 & 36.5 & 0.55 & 0.5882 \\
\hline MDate & 19360 & 0.04665 & 0.7705 & 19.4 & 0.06 & 0.9523 \\
\hline MDate & 19361 & 0.2883 & 0.6863 & 36.5 & 0.42 & 0.6769 \\
\hline MDate & 19362 & -0.4039 & 0.7705 & 19.4 & -0.52 & 0.6061 \\
\hline MDate & 19363 & -0.2170 & 0.6863 & 36.5 & -0.32 & 0.7537 \\
\hline MDate & 19364 & -0.7418 & 0.7705 & 19.4 & -0.96 & 0.3475 \\
\hline MDate & 19365 & -0.7981 & 0.7705 & 19.4 & -1.04 & 0.3130 \\
\hline MDate & 19366 & -0.5165 & 0.7705 & 19.4 & -0.67 & 0.5105 \\
\hline MDate & 19369 & 0.1781 & 0.7705 & 19.4 & 0.23 & 0.8197 \\
\hline MDate & 19371 & -0.2725 & 0.7705 & 19.4 & -0.35 & 0.7274 \\
\hline MDate & 19372 & -2.2236 & 0.6863 & 36.5 & -3.24 & 0.0025 \\
\hline MDate & 19373 & -1.4176 & 0.7705 & 19.4 & -1.84 & 0.0811 \\
\hline MDate & 19374 & -0.3288 & 0.7705 & 19.4 & -0.43 & 0.6743 \\
\hline MDate & 19375 & -0.3288 & 0.7705 & 19.4 & -0.43 & 0.6743 \\
\hline MDate & 19376 & -0.2996 & 0.6208 & 58.2 & -0.48 & 0.6312 \\
\hline MDate & 19377 & 0.3572 & 0.6208 & 58.2 & 0.58 & 0.5672 \\
\hline MDate & 19378 & -0.2537 & 0.7705 & 19.4 & -0.33 & 0.7455 \\
\hline MDate & 19379 & -0.6983 & 0.6208 & 58.2 & -1.12 & 0.2652 \\
\hline MDate & 19380 & -0.2527 & 0.6208 & 58.2 & -0.41 & 0.6855 \\
\hline MDate & 19381 & -0.4153 & 0.5700 & 81.5 & -0.73 & 0.4683 \\
\hline MDate & 19382 & 0.7358 & 0.6863 & 36.5 & 1.07 & 0.2907 \\
\hline MDate & 19383 & 0.6738 & 0.6208 & 58.2 & 1.09 & 0.2822 \\
\hline MDate & 19384 & 0.8052 & 0.5298 & 103 & 1.52 & 0.1316 \\
\hline MDate & 19385 & 0.7647 & 0.6863 & 36.5 & 1.11 & 0.2724 \\
\hline MDate & 19386 & -0.08848 & 0.6208 & 58.2 & -0.14 & 0.8872 \\
\hline MDate & 19387 & 0.4615 & 0.6863 & 36.5 & 0.67 & 0.5055 \\
\hline MDate & 19388 & 0.4409 & 0.7705 & 19.4 & 0.57 & 0.5737 \\
\hline MDate & 19389 & -0.2170 & 0.6863 & 36.5 & -0.32 & 0.7537 \\
\hline MDate & 19390 & 0.06687 & 0.4973 & 121 & 0.13 & 0.8933 \\
\hline MDate & 19391 & 0.3658 & 0.7705 & 19.4 & 0.47 & 0.6403 \\
\hline MDate & 19392 & 0.7911 & 0.6208 & 58.2 & 1.27 & 0.2076 \\
\hline MDate & 19393 & -0.3180 & 0.6863 & 36.5 & -0.46 & 0.6458 \\
\hline MDate & 19394 & 0.2532 & 0.7705 & 19.4 & 0.33 & 0.7460 \\
\hline MDate & 19395 & -0.2349 & 0.7705 & 19.4 & -0.30 & 0.7637 \\
\hline MDate & 19396 & -0.08705 & 0.6863 & 36.5 & -0.13 & 0.8998 \\
\hline MDate & 19397 & 0.2156 & 0.7705 & 19.4 & 0.28 & 0.7826 \\
\hline
\end{tabular}

The SAS System

09:55 Thursday, March 14, 201319

Analyte $=$ Nitrate RefValue $=16$

The Mixed Procedure

Solution for Random Effects

$\begin{array}{lrrrrrr}\text { Effect } & \text { MDate } & \text { Estimate } & \begin{array}{r}\text { Std Err } \\ \text { Pred }\end{array} & \text { DF } & \text { t Value } & \text { Pr > |t } \\ \text { MDate } & 19398 & 0.2164 & 0.6208 & 58.2 & 0.35 & 0.7286 \\ \text { MDate } & 19399 & 0.3282 & 0.7705 & 19.4 & 0.43 & 0.6748\end{array}$

The SAS System

09:55 Thursday, March 14, 201320

Analyte $=$ Sol0 $x$ RefValue $=2$

The Mixed Procedure 
SRNL-STI-2013-00139

Revision 0

Model Information

\begin{abstract}
Data Set
Dependent Variable Covariance Structure

Estimation Method Residual Variance Method Fixed Effects SE Method

Degrees of Freedom Method
\end{abstract}

WORK. TOMSORT

PctDiff

Variance Components

REML

Profile

Prasad-Rao-Jeske-

Kackar-Harville

Kenward-Roger

Class Level Information

\begin{tabular}{lllllll} 
Class Levels & \multicolumn{2}{l}{ Values } \\
MDate & 81 & 19306 & 19307 & 19308 & 19310 & 19311 \\
& 19313 & 19314 & 19315 & 19316 & 19317 \\
& 19318 & 19319 & 19321 & 19322 & 19323 \\
19324 & 19325 & 19326 & 19327 & 19329 \\
19330 & 19331 & 19333 & 19334 & 19335 \\
19336 & 19337 & 19338 & 19339 & 19341 \\
19342 & 19343 & 19344 & 19345 & 19347 \\
19348 & 19349 & 19350 & 19351 & 19352 \\
19354 & 19355 & 19356 & 19357 & 19358 \\
19359 & 19360 & 19361 & 19362 & 19363 \\
19364 & 19366 & 19369 & 19370 & 19371 \\
19372 & 19373 & 19375 & 19376 & 19377 \\
19378 & 19379 & 19380 & 19381 & 19382 \\
& 19383 & 19384 & 19385 & 19386 & 19387 \\
19388 & 19389 & 19390 & 19392 & 19393 \\
19394 & 19395 & 19396 & 19397 & 19398 \\
& 19399 & & & &
\end{tabular}

Dimensions

Covariance Parameters

Columns in $X$

Columns in $Z$

Subjects

Max Obs Per Subject

Observations Used

Observations Not Used

Total Observations
2
1
81
1
142
142
0
142

09:55 Thursday, March 14, 2013

Analyte $=$ Sol0x RefValue $=2$

The Mixed Procedure

Iteration History

Iteration Evaluations -2 Res Log Like Criterion 
SRNL-STI-2013-00139

Revision 0

$\begin{array}{llll}0 & 1 & 728.36964229 & \\ 1 & 3 & 683.02847649 & 0.00013228 \\ 2 & 1 & 683.00164361 & 0.00000050 \\ 3 & 1 & 683.00153828 & 0.00000000\end{array}$

Convergence criteria met.

Covariance Parameter Estimates

$\begin{array}{lrrrr}\text { Cov Parm } & \text { Estimate } & \text { Alpha } & \text { Lower } & \text { Upper } \\ & & & & \\ \text { MDate } & 7.0916 & 0.05 & 4.1459 & 10.0373 \\ \text { Residual } & 2.9350 & 0.05 & 2.1127 & 4.3542\end{array}$

Asymptotic Covariance Matrix of Estimates

$\begin{array}{rlrr}\text { Row } & \text { Cov Parm } & \text { CovP1 } & \text { CovP2 } \\ & & & \\ 1 & \text { MDate } & 2.2589 & -0.2116 \\ 2 & \text { Residual } & -0.2116 & 0.2884\end{array}$

Fit Statistics

$\begin{array}{ll}-2 \text { Res Log Likelihood } & 683.0 \\ \text { AIC (smaller is better) } & 687.0 \\ \text { AICC (smaller is better) } & 687.1 \\ \text { BIC (smaller is better) } & 691.8\end{array}$

Null Model Likelihood Ratio Test

DF Chi-Square $\quad \mathrm{Pr}>\mathrm{ChiSq}$

$1 \quad 45.37<.0001$

The SAS System 09:55 Thursday, March 14, 201322

Analyte=Solox RefValue $=2$

The Mixed Procedure

Solution for Fixed Effects

\begin{tabular}{|c|c|c|c|c|c|}
\hline \multicolumn{6}{|c|}{ Standard } \\
\hline Effect & Estimate & Error & DF & t value & $\operatorname{Pr}>|t|$ \\
\hline Intercept & 3.8597 & 0.3354 & 77.3 & 11.51 & $<.0001$ \\
\hline
\end{tabular}

Solution for Random Effects 
SRNL-STI-2013-00139

Revision 0

\begin{tabular}{|c|c|c|c|c|c|c|}
\hline \multirow{2}{*}{ Effect } & \multicolumn{5}{|c|}{ Std Err } & \multirow[b]{2}{*}{$\mathrm{Pr}>|\mathrm{t}|$} \\
\hline & MDate & Estimate & Pred & DF & t Value & \\
\hline MDate & 19306 & 2.2211 & 1.4885 & 128 & 1.49 & 0.1381 \\
\hline MDate & 19307 & 1.1601 & 1.4885 & 128 & 0.78 & 0.4372 \\
\hline MDate & 19308 & 0.4529 & 1.4885 & 128 & 0.30 & 0.7614 \\
\hline MDate & 19310 & -1.1266 & 1.1519 & 107 & -0.98 & 0.3303 \\
\hline MDate & 19311 & -0.9617 & 1.4885 & 128 & -0.65 & 0.5194 \\
\hline MDate & 19313 & -0.7123 & 1.1519 & 107 & -0.62 & 0.5376 \\
\hline MDate & 19314 & -3.0835 & 1.4885 & 128 & -2.07 & 0.0403 \\
\hline MDate & 19315 & -1.6690 & 1.4885 & 128 & -1.12 & 0.2643 \\
\hline MDate & 19316 & -1.3153 & 1.4885 & 128 & -0.88 & 0.3785 \\
\hline MDate & 19317 & -1.6690 & 1.4885 & 128 & -1.12 & 0.2643 \\
\hline MDate & 19318 & 0.5305 & 1.1519 & 107 & 0.46 & 0.6461 \\
\hline MDate & 19319 & -3.0835 & 1.4885 & 128 & -2.07 & 0.0403 \\
\hline MDate & 19321 & -5.0837 & 0.8768 & 100 & -5.80 & $<.0001$ \\
\hline MDate & 19322 & -3.6122 & 1.1519 & 107 & -3.14 & 0.0022 \\
\hline MDate & 19323 & 4.8804 & 1.1519 & 107 & 4.24 & $<.0001$ \\
\hline MDate & 19324 & 5.0502 & 1.4885 & 128 & 3.39 & 0.0009 \\
\hline MDate & 19325 & 4.0518 & 1.1519 & 107 & 3.52 & 0.0006 \\
\hline MDate & 19326 & 4.6966 & 1.4885 & 128 & 3.16 & 0.0020 \\
\hline MDate & 19327 & 1.1601 & 1.4885 & 128 & 0.78 & 0.4372 \\
\hline MDate & 19329 & 0.9448 & 1.1519 & 107 & 0.82 & 0.4139 \\
\hline MDate & 19330 & 0.9448 & 1.1519 & 107 & 0.82 & 0.4139 \\
\hline MDate & 19331 & -4.0265 & 1.1519 & 107 & -3.50 & 0.0007 \\
\hline MDate & 19333 & -0.5052 & 1.1519 & 107 & -0.44 & 0.6619 \\
\hline MDate & 19334 & -4.8518 & 1.4885 & 128 & -3.26 & 0.0014 \\
\hline MDate & 19335 & -0.9617 & 1.4885 & 128 & -0.65 & 0.5194 \\
\hline MDate & 19336 & -0.9617 & 1.4885 & 128 & -0.65 & 0.5194 \\
\hline MDate & 19337 & -0.2544 & 1.4885 & 128 & -0.17 & 0.8645 \\
\hline MDate & 19338 & -3.4372 & 1.4885 & 128 & -2.31 & 0.0225 \\
\hline MDate & 19339 & -3.4372 & 1.4885 & 128 & -2.31 & 0.022 \\
\hline MDate & 19341 & -0.2980 & 1.1519 & 107 & -0.26 & 0.7963 \\
\hline MDate & 19342 & -1.7807 & 0.9822 & 101 & -1.81 & 0.0728 \\
\hline MDate & 19343 & -0.6081 & 1.4885 & 128 & -0.41 & 0.6836 \\
\hline MDate & 19344 & -0.6081 & 1.4885 & 128 & -0.41 & 0.683 \\
\hline MDate & 19345 & -1.3153 & 1.4885 & 128 & -0.88 & 0.3785 \\
\hline MDate & 19347 & 0.1162 & 1.1519 & 107 & 0.10 & 0.9198 \\
\hline MDate & 19348 & -0.5052 & 1.1519 & 107 & -0.44 & 0.6619 \\
\hline
\end{tabular}

The SAS System

09:55 Thursday, March 14, 201323

Analyte=Sol0x RefValue $=2$

The Mixed Procedure

Solution for Random Effects

$\begin{array}{lrrrrrr}\text { Effect } & \text { MDate } & \text { Estimate } & \begin{array}{r}\text { Std Err } \\ \text { Pred }\end{array} & \text { DF } & t \text { Value } & \text { Pr > }|t| \\ \text { MDate } & 19349 & 0.8065 & 1.4885 & 128 & 0.54 & 0.5889 \\ \text { MDate } & 19350 & -0.09943 & 0.8768 & 100 & -0.11 & 0.9099 \\ \text { MDate } & 19351 & -1.3337 & 1.1519 & 107 & -1.16 & 0.2495 \\ \text { MDate } & 19352 & -1.1266 & 1.1519 & 107 & -0.98 & 0.3303 \\ \text { MDate } & 19354 & -0.6081 & 1.4885 & 128 & -0.41 & 0.6836 \\ \text { MDate } & 19355 & 0.5305 & 1.1519 & 107 & 0.46 & 0.6461\end{array}$


SRNL-STI-2013-00139

Revision 0

\begin{tabular}{|c|c|c|c|c|c|c|}
\hline MDate & 19356 & -0.7555 & 0.9822 & 101 & -0.77 & 0.4436 \\
\hline MDate & 19357 & 0.09922 & 1.4885 & 128 & 0.07 & 0.9470 \\
\hline MDate & 19358 & -1.1266 & 1.1519 & 107 & -0.98 & 0.3303 \\
\hline MDate & 19359 & -0.09091 & 1.1519 & 107 & -0.08 & 0.9372 \\
\hline MDate & 19360 & -0.2544 & 1.4885 & 128 & -0.17 & 0.8645 \\
\hline MDate & 19361 & -1.1266 & 1.1519 & 107 & -0.98 & 0.3303 \\
\hline MDate & 19362 & -2.0226 & 1.4885 & 128 & -1.36 & 0.1766 \\
\hline MDate & 19363 & -1.1266 & 1.1519 & 107 & -0.98 & 0.3303 \\
\hline MDate & 19364 & -2.7299 & 1.4885 & 128 & -1.83 & 0.0690 \\
\hline MDate & 19366 & -3.7908 & 1.4885 & 128 & -2.55 & 0.0121 \\
\hline MDate & 19369 & -1.3153 & 1.4885 & 128 & -0.88 & 0.3785 \\
\hline MDate & 19370 & -5.2054 & 1.4885 & 128 & -3.50 & 0.0006 \\
\hline MDate & 19371 & -2.3666 & 0.9822 & 101 & -2.41 & 0.0178 \\
\hline MDate & 19372 & -1.1949 & 0.9822 & 101 & -1.22 & 0.2266 \\
\hline MDate & 19373 & 2.5747 & 1.4885 & 128 & 1.73 & 0.0861 \\
\hline MDate & 19375 & 2.5747 & 1.4885 & 128 & 1.73 & 0.0861 \\
\hline MDate & 19376 & 2.4667 & 0.9822 & 101 & 2.51 & 0.0136 \\
\hline MDate & 19377 & 3.2233 & 1.1519 & 107 & 2.80 & 0.0061 \\
\hline MDate & 19378 & 2.9283 & 1.4885 & 128 & 1.97 & 0.0513 \\
\hline MDate & 19379 & 1.5138 & 1.4885 & 128 & 1.02 & 0.3111 \\
\hline MDate & 19380 & 1.5662 & 1.1519 & 107 & 1.36 & 0.1768 \\
\hline MDate & 19381 & 0.3537 & 0.8768 & 100 & 0.40 & 0.6875 \\
\hline MDate & 19382 & -0.5052 & 1.1519 & 107 & -0.44 & 0.6619 \\
\hline MDate & 19383 & 1.2950 & 0.9822 & 101 & 1.32 & 0.1904 \\
\hline MDate & 19384 & 4.4703 & 0.8037 & 102 & 5.56 & $<.0001$ \\
\hline MDate & 19385 & 4.0518 & 1.1519 & 107 & 3.52 & 0.0006 \\
\hline MDate & 19386 & 2.3202 & 0.9822 & 101 & 2.36 & 0.0201 \\
\hline MDate & 19387 & 2.5747 & 1.4885 & 128 & 1.73 & 0.0861 \\
\hline MDate & 19388 & 1.8674 & 1.4885 & 128 & 1.25 & 0.2119 \\
\hline MDate & 19389 & 2.7596 & 0.9822 & 101 & 2.81 & 0.0060 \\
\hline MDate & 19390 & -1.7986 & 0.8768 & 100 & -2.05 & 0.0428 \\
\hline MDate & 19392 & 0.4529 & 1.4885 & 128 & 0.30 & 0.7614 \\
\hline MDate & 19393 & -0.5052 & 1.1519 & 107 & -0.44 & 0.6619 \\
\hline MDate & 19394 & -0.7123 & 1.1519 & 107 & -0.62 & 0.5376 \\
\hline MDate & 19395 & 1.5138 & 1.4885 & 128 & 1.02 & 0.3111 \\
\hline MDate & 19396 & 2.3947 & 1.1519 & 107 & 2.08 & 0.0400 \\
\hline MDate & 19397 & 1.8674 & 1.4885 & 128 & 1.25 & 0.2119 \\
\hline MDate & 19398 & 2.3202 & 0.9822 & 101 & 2.36 & 0.0201 \\
\hline
\end{tabular}

The SAS System

09:55 Thursday, March 14, 201324

Analyte $=$ Sol0 $x$ RefValue $=2$

The Mixed Procedure

Solution for Random Effects

$\begin{array}{lrrrrrr}\text { Effect } & \text { MDate } & \text { Estimate } & \begin{array}{r}\text { Std Err } \\ \text { Pred }\end{array} & \text { DF } & \text { t Value } & \text { Pr }>|t| \\ \text { MDate } & 19399 & 2.9283 & 1.4885 & 128 & 1.97 & 0.0513\end{array}$

The SAS System

09:55 Thursday, March 14, 201325

Analyte $=$ Sol0 $x$ RefValue $=16$

The Mixed Procedure 
SRNL-STI-2013-00139

Revision 0

Model Information

\begin{abstract}
Data Set
Dependent Variable Covariance Structure

Estimation Method Residual Variance Method Fixed Effects SE Method

Degrees of Freedom Method
\end{abstract}

WORK. TOMSORT

PctDiff

Variance Components

REML

Profile

Prasad-Rao-Jeske-

Kackar-Harville

Kenward-Roger

Class Level Information

\begin{tabular}{lllllll} 
Class Levels & \multicolumn{2}{l}{ Values } \\
MDate 80 & 19306 & 19307 & 19308 & 19310 & 19311 \\
& 19313 & 19314 & 19315 & 19316 & 19317 \\
19318 & 19319 & 19321 & 19322 & 19323 \\
19324 & 19325 & 19326 & 19327 & 19329 \\
19330 & 19331 & 19333 & 19335 & 19336 \\
19337 & 19338 & 19339 & 19341 & 19342 \\
19343 & 19344 & 19345 & 19347 & 19348 \\
19349 & 19350 & 19351 & 19352 & 19354 \\
19355 & 19356 & 19357 & 19358 & 19359 \\
19360 & 19361 & 19362 & 19363 & 19364 \\
19365 & 19366 & 19369 & 19371 & 19372 \\
19373 & 19375 & 19376 & 19377 & 19378 \\
19379 & 19380 & 19381 & 19382 & 19383 \\
19384 & 19385 & 19386 & 19387 & 19388 \\
& 19389 & 19390 & 19392 & 19393 & 19394 \\
19395 & 19396 & 19397 & 19398 & 19399
\end{tabular}

Dimensions

Covariance Parameters

Columns in $X$

Columns in $Z$

Subjects

Max Obs Per Subject

Observations Used

Observations Not Used

Total Observations

2
1
80
1
137
137
0
137

2

1

80

137

137

137

The SAS System

09:55 Thursday, March 14, 2013

Analyte $=$ Sol0x RefValue $=16$

The Mixed Procedure

Iteration History

Iteration Evaluations -2 Res Log Like Criterion 
SRNL-STI-2013-00139

Revision 0

$\begin{array}{llll}0 & 1 & 588.87330476 & \\ 1 & 2 & 575.92581417 & 0.00000229 \\ 2 & 1 & 575.92543964 & 0.00000000\end{array}$

Convergence criteria met.

Covariance Parameter Estimates

$\begin{array}{lrrrr}\text { Cov Parm } & \text { Estimate } & \text { Alpha } & \text { Lower } & \text { Upper } \\ & & & & \\ \text { MDate } & 1.9147 & 0.05 & 0.6491 & 3.1802 \\ \text { Residual } & 2.4368 & 0.05 & 1.7403 & 3.6565\end{array}$

Asymptotic Covariance Matrix of Estimates

$\begin{array}{rlrr}\text { Row } & \text { Cov Parm } & \text { CovP1 } & \text { CovP2 } \\ & & & \\ 1 & \text { MDate } & 0.4169 & -0.1419 \\ 2 & \text { Residual } & -0.1419 & 0.2093\end{array}$

Fit Statistics

$\begin{array}{ll}-2 \text { Res Log Likelihood } & 575.9 \\ \text { AIC (smaller is better) } & 579.9 \\ \text { AICC (smaller is better) } & 580.0 \\ \text { BIC (smaller is better) } & 584.7\end{array}$

Null Model Likelihood Ratio Test

DF Chi-Square $\quad \mathrm{Pr}>\mathrm{ChiSq}$

12.95

The SAS System

09:55 Thursday, March 14, 201327

Analyte $=$ Sol0x RefValue $=16$

The Mixed Procedure

Solution for Fixed Effects

$\begin{array}{lrrrrr} & \text { Estimate } & \begin{array}{r}\text { Standard } \\ \text { Error }\end{array} & \text { DF } & t \text { Value } & \operatorname{Pr}>|t| \\ \text { Intercept } & -0.2165 & 0.2113 & 71.8 & -1.02 & 0.3091\end{array}$

Solution for Random Effects 
SRNL-STI-2013-00139

Revision 0

\begin{tabular}{|c|c|c|c|c|c|c|}
\hline & & & sta Err & & & \\
\hline Effect & MDate & Estimate & Pred & DF & $t$ Value & $\operatorname{Pr}>|t|$ \\
\hline MDate & 19306 & 0.8378 & 1.0909 & 70.3 & 0.77 & 0.4451 \\
\hline MDate & 19307 & 0.06775 & 1.0909 & 70.3 & 0.06 & 0.9507 \\
\hline MDate & 19308 & -0.2073 & 1.0909 & 70.3 & -0.19 & 0.8499 \\
\hline MDate & 19310 & -1.0327 & 0.9136 & 120 & -1.13 & 0.2606 \\
\hline MDate & 19311 & -0.9223 & 1.0909 & 70.3 & -0.85 & 0.4007 \\
\hline MDate & 19313 & -0.05869 & 0.9136 & 120 & -0.06 & 0.9489 \\
\hline MDate & 19314 & 0.04025 & 1.0909 & 70.3 & 0.04 & 0.9707 \\
\hline MDate & 19315 & -0.4273 & 1.0909 & 70.3 & -0.39 & 0.6965 \\
\hline MDate & 19316 & -0.2073 & 1.0909 & 70.3 & -0.19 & 0.8499 \\
\hline MDate & 19317 & -0.3998 & 1.0909 & 70.3 & -0.37 & 0.7151 \\
\hline MDate & 19318 & 0.2469 & 0.9136 & 120 & 0.27 & 0.7875 \\
\hline MDate & 19319 & -1.8298 & 1.0909 & 70.3 & -1.68 & 0.0979 \\
\hline MDate & 19321 & -0.5707 & 0.7237 & 134 & -0.79 & 0.4317 \\
\hline MDate & 19322 & -0.8948 & 1.0909 & 70.3 & -0.82 & 0.4149 \\
\hline MDate & 19323 & -1.2236 & 0.9136 & 120 & -1.34 & 0.1830 \\
\hline MDate & 19324 & 0.5353 & 1.0909 & 70.3 & 0.49 & 0.6252 \\
\hline MDate & 19325 & 0.7225 & 0.8015 & 135 & 0.90 & 0.3689 \\
\hline MDate & 19326 & 0.6453 & 1.0909 & 70.3 & 0.59 & 0.5561 \\
\hline MDate & 19327 & -1.3073 & 1.0909 & 70.3 & -1.20 & 0.2348 \\
\hline MDate & 19329 & -0.4024 & 0.9136 & 120 & -0.44 & 0.6604 \\
\hline MDate & 19330 & -0.5934 & 0.9136 & 120 & -0.65 & 0.5172 \\
\hline MDate & 19331 & -0.1542 & 0.9136 & 120 & -0.17 & 0.8663 \\
\hline MDate & 19333 & 0.4187 & 0.9136 & 120 & 0.46 & 0.6475 \\
\hline MDate & 19335 & -1.0323 & 1.0909 & 70.3 & -0.95 & 0.3473 \\
\hline MDate & 19336 & 0.04025 & 1.0909 & 70.3 & 0.04 & 0.9707 \\
\hline MDate & 19337 & 0.3977 & 1.0909 & 70.3 & 0.36 & 0.7165 \\
\hline MDate & 19338 & -1.0048 & 1.0909 & 70.3 & -0.92 & 0.3602 \\
\hline MDate & 19339 & -1.7198 & 1.0909 & 70.3 & -1.58 & 0.1194 \\
\hline MDate & 19341 & 0.1514 & 0.9136 & 120 & 0.17 & 0.8687 \\
\hline MDate & 19342 & -0.7842 & 0.8015 & 135 & -0.98 & 0.3296 \\
\hline MDate & 19343 & -0.3173 & 1.0909 & 70.3 & -0.29 & 0.7720 \\
\hline MDate & 19344 & -0.3998 & 1.0909 & 70.3 & -0.37 & 0.7151 \\
\hline MDate & 19345 & -0.2898 & 1.0909 & 70.3 & -0.27 & 0.7913 \\
\hline MDate & 19347 & -1.1090 & 0.9136 & 120 & -1.21 & 0.2272 \\
\hline MDate & 19348 & 0.09409 & 0.9136 & 120 & 0.10 & 0.9181 \\
\hline MDate & 19349 & 0.2327 & 1.0909 & 70.3 & 0.21 & 0.8317 \\
\hline
\end{tabular}

The SAS System

09:55 Thursday, March 14, 201328

Analyte $=$ Sol0x RefValue $=16$

The Mixed Procedure

Solution for Random Effects

$\begin{array}{lrrrrrr}\text { Effect } & \text { MDate } & \text { Estimate } & \begin{array}{r}\text { Std Err } \\ \text { Pred }\end{array} & \text { DF } & t \text { Value } & \text { Pr > }|t| \\ \text { MDate } & 19350 & -0.3810 & 0.7237 & 134 & -0.53 & 0.5994 \\ \text { MDate } & 19351 & 0.2087 & 0.9136 & 120 & 0.23 & 0.8197 \\ \text { MDate } & 19352 & -0.5648 & 1.0909 & 70.3 & -0.52 & 0.6063 \\ \text { MDate } & 19354 & 0.3152 & 1.0909 & 70.3 & 0.29 & 0.7734 \\ \text { MDate } & 19355 & -0.3746 & 0.8015 & 135 & -0.47 & 0.6410 \\ \text { MDate } & 19356 & 0.005711 & 0.8015 & 135 & 0.01 & 0.9943\end{array}$


SRNL-STI-2013-00139

Revision 0

\begin{tabular}{|c|c|c|c|c|c|c|}
\hline MDate & 19357 & 0.3427 & 1.0909 & 70.3 & 0.31 & 0.7543 \\
\hline MDate & 19358 & 0.3233 & 0.9136 & 120 & 0.35 & 0.7241 \\
\hline MDate & 19359 & 0.01770 & 0.9136 & 120 & 0.02 & 0.9846 \\
\hline MDate & 19360 & -0.3173 & 1.0909 & 70.3 & -0.29 & 0.7720 \\
\hline MDate & 19361 & -0.4788 & 0.9136 & 120 & -0.52 & 0.6012 \\
\hline MDate & 19362 & -1.0873 & 1.0909 & 70.3 & -1.00 & 0.3223 \\
\hline MDate & 19363 & -1.0517 & 0.9136 & 120 & -1.15 & 0.2519 \\
\hline MDate & 19364 & -1.5823 & 1.0909 & 70.3 & -1.45 & 0.1514 \\
\hline MDate & 19365 & -1.7198 & 1.0909 & 70.3 & -1.58 & 0.1194 \\
\hline MDate & 19366 & -1.3898 & 1.0909 & 70.3 & -1.27 & 0.2069 \\
\hline MDate & 19369 & -0.4273 & 1.0909 & 70.3 & -0.39 & 0.6965 \\
\hline MDate & 19371 & -0.8673 & 1.0909 & 70.3 & -0.79 & 0.4293 \\
\hline MDate & 19372 & -2.1873 & 1.0909 & 70.3 & -2.01 & 0.0488 \\
\hline MDate & 19373 & -2.2423 & 1.0909 & 70.3 & -2.06 & 0.0436 \\
\hline MDate & 19375 & 0.6453 & 1.0909 & 70.3 & 0.59 & 0.5561 \\
\hline MDate & 19376 & 0.5323 & 0.8015 & 135 & 0.66 & 0.5077 \\
\hline MDate & 19377 & 1.9465 & 0.9136 & 120 & 2.13 & 0.0352 \\
\hline MDate & 19378 & 0.7003 & 1.0909 & 70.3 & 0.64 & 0.5230 \\
\hline MDate & 19379 & 0.3977 & 1.0909 & 70.3 & 0.36 & 0.7165 \\
\hline MDate & 19380 & 1.2017 & 0.9136 & 120 & 1.32 & 0.1909 \\
\hline MDate & 19381 & 1.3970 & 0.7237 & 134 & 1.93 & 0.0557 \\
\hline MDate & 19382 & 2.3476 & 0.9136 & 120 & 2.57 & 0.0114 \\
\hline MDate & 19383 & 2.3023 & 0.8015 & 135 & 2.87 & 0.0047 \\
\hline MDate & 19384 & 2.3247 & 0.6662 & 130 & 3.49 & 0.0007 \\
\hline MDate & 19385 & 1.9656 & 0.9136 & 120 & 2.15 & 0.0334 \\
\hline MDate & 19386 & 0.9565 & 0.8015 & 135 & 1.19 & 0.2348 \\
\hline MDate & 19387 & 1.1128 & 1.0909 & 70.3 & 1.02 & 0.3112 \\
\hline MDate & 19388 & 1.0303 & 1.0909 & 70.3 & 0.94 & 0.3482 \\
\hline MDate & 19389 & 0.8249 & 0.8015 & 135 & 1.03 & 0.3052 \\
\hline MDate & 19390 & 0.3302 & 0.7237 & 134 & 0.46 & 0.6490 \\
\hline MDate & 19392 & 1.2228 & 1.0909 & 70.3 & 1.12 & 0.2662 \\
\hline MDate & 19393 & 0.3996 & 0.9136 & 120 & 0.44 & 0.6626 \\
\hline MDate & 19394 & -0.4597 & 0.9136 & 120 & -0.50 & 0.6158 \\
\hline MDate & 19395 & 0.3427 & 1.0909 & 70.3 & 0.31 & 0.7543 \\
\hline MDate & 19396 & 0.8580 & 0.9136 & 120 & 0.94 & 0.3496 \\
\hline MDate & 19397 & 1.0303 & 1.0909 & 70.3 & 0.94 & 0.3482 \\
\hline MDate & 19398 & 1.3661 & 0.8015 & 135 & 1.70 & 0.0906 \\
\hline MDate & 19399 & 1.1403 & 1.0909 & 70.3 & 1.05 & 0.2995 \\
\hline
\end{tabular}

The SAS System

Analyte $=$ TOC RefValue $=$

The Mixed Procedure

Model Information

Data Set

Dependent Variable

Covariance Structure

Estimation Method

Residual Variance Method

Fixed Effects SE Method

Degrees of Freedom Method
WORK. TOMSORT

PctDiff

Variance Components

REML

Profile

Prasad-Rao-Jeske-

Kackar-Harville

Kenward-Roger 
SRNL-STI-2013-00139

Revision 0

Class Level Information

$\begin{array}{lllllll}\text { Class } & \text { Levels } & \text { Values } \\ \text { MDate } & 21 & 19281 & 19290 & 19318 & 19322 & 19331 \\ & & 19333 & 19336 & 19340 & 19341 & 19350 \\ & 19351 & 19352 & 19370 & 19371 & 19372 \\ & 19380 & 19381 & 19384 & 19386 & 19390 \\ & & 19398 & & & & \end{array}$

Dimensions

Covariance Parameters

2

Columns in $X$

Columns in $Z$

Subjects

Max Obs Per Subject

Observations Used

Observations Not Used

Total Observations

1

21

1

91

91

0

91

Iteration History

$\begin{array}{rrrr}\text { Iteration } & \text { Evaluations } & -2 \text { Res Log Like } & \text { Criterion } \\ 0 & 1 & 484.89577517 & \\ 1 & 2 & 461.14220600 & 0.00025960 \\ 2 & 1 & 461.10107368 & 0.00000359 \\ 3 & 1 & 461.10053755 & 0.00000000\end{array}$

Convergence criteria met.

The SAS System

09:55 Thursday, March 14, 201330

Analyte $=T O C$ RefValue $=1$

The Mixed Procedure

Covariance Parameter Estimates

$\begin{array}{lrrrr}\text { Cov Parm } & \text { Estimate } & \text { Alpha } & \text { Lower } & \text { Upper } \\ & & & & \\ \text { MDate } & 6.3409 & 0.05 & 1.3235 & 11.3584 \\ \text { Residual } & 6.5042 & 0.05 & 4.7850 & 9.3556\end{array}$

Asymptotic Covariance Matrix of Estimates

$\begin{array}{clrr}\text { Row } & \text { Cov Parm } & \text { CovP1 } & \text { CovP2 } \\ & & & \\ 1 & \text { MDate } & 6.5534 & -0.3428 \\ 2 & \text { Residual } & -0.3428 & 1.2203\end{array}$


SRNL-STI-2013-00139

Revision 0

Fit Statistics

$\begin{array}{ll}-2 \text { Res Log Likelihood } & 461.1 \\ \text { AIC (smaller is better) } & 465.1 \\ \text { AICC (smaller is better) } & 465.2 \\ \text { BIC (smaller is better) } & 467.2\end{array}$

Null Model Likelihood Ratio Test

$$
\begin{aligned}
& \text { DF Chi-Square } \quad \mathrm{Pr}>\mathrm{ChiSq} \\
& 1 \quad 23.80<.0001
\end{aligned}
$$

Solution for Fixed Effects

$\begin{array}{lrrrrr}\text { Effect } & \text { Estimate } & \begin{array}{r}\text { Standard } \\ \text { Error }\end{array} & \text { DF } & t \text { Value } & \operatorname{Pr}>|t| \\ \text { Intercept } & -0.2413 & 0.6137 & 19.3 & -0.39 & 0.6985\end{array}$

Solution for Random Effects

$\begin{array}{lrrrrrr}\text { Effect } & \text { MDate } & \text { Estimate } & \begin{array}{r}\text { Std Err } \\ \text { Pred }\end{array} & \text { DF } & t \text { Value } & \text { Pr }>|t| \\ \text { MDate } & 19281 & -0.6038 & 1.2712 & 68.6 & -0.48 & 0.6363 \\ \text { MDate } & 19290 & -1.9967 & 1.2712 & 68.6 & -1.57 & 0.1209 \\ \text { MDate } & 19318 & -2.7926 & 1.2712 & 68.6 & -2.20 & 0.0314 \\ \text { MDate } & 19322 & -0.00691 & 1.2712 & 68.6 & -0.01 & 0.9957 \\ \text { MDate } & 19331 & 3.7736 & 1.2712 & 68.6 & 2.97 & 0.0041\end{array}$

The SAS System

09:55 Thursday, March 14, 201331

Analyte $=$ TOC RefValue $=1$

The Mixed Procedure

Solution for Random Effects

$\begin{array}{lrrrrrr}\text { Effect } & \text { MDate } & \text { Estimate } & \begin{array}{r}\text { Std Err } \\ \text { Pred }\end{array} & \text { DF } & t \text { Value } & \text { Pr }>|t| \\ \text { MDate } & 19333 & 1.5849 & 1.2712 & 68.6 & 1.25 & 0.2167 \\ \text { MDate } & 19336 & -0.4049 & 1.2712 & 68.6 & -0.32 & 0.7511 \\ \text { MDate } & 19340 & 0.3910 & 1.2712 & 68.6 & 0.31 & 0.7593 \\ \text { MDate } & 19341 & -0.2059 & 1.2712 & 68.6 & -0.16 & 0.8718 \\ \text { MDate } & 19350 & -1.3997 & 1.2712 & 68.6 & -1.10 & 0.2747 \\ \text { MDate } & 19351 & -1.0018 & 1.2712 & 68.6 & -0.79 & 0.4334 \\ \text { MDate } & 19352 & 1.9828 & 1.2712 & 68.6 & 1.56 & 0.1234 \\ \text { MDate } & 19370 & -2.1956 & 1.2712 & 68.6 & -1.73 & 0.0886 \\ \text { MDate } & 19371 & -2.3946 & 1.2712 & 68.6 & -1.88 & 0.0638 \\ \text { MDate } & 19372 & -2.5560 & 1.0235 & 65.1 & -2.50 & 0.0151 \\ \text { MDate } & 19380 & 4.3705 & 1.2712 & 68.6 & 3.44 & 0.0010 \\ \text { MDate } & 19381 & -2.1956 & 1.2712 & 68.6 & -1.73 & 0.0886\end{array}$


SRNL-STI-2013-00139

Revision 0

$\begin{array}{lrrrrrr}\text { MDate } & 19384 & 0.3910 & 1.2712 & 68.6 & 0.31 & 0.7593 \\ \text { MDate } & 19386 & 0.5900 & 1.2712 & 68.6 & 0.46 & 0.6440 \\ \text { MDate } & 19390 & -0.2293 & 1.0235 & 65.1 & -0.22 & 0.8234 \\ \text { MDate } & 19398 & 4.8994 & 1.3944 & 65.8 & 3.51 & 0.0008\end{array}$

The SAS System

09:55 Thursday, March 14, 201332

Analyte $=T O C$ RefValue $=20$

The Mixed Procedure

Model Information

Data Set

Dependent Variable

Covariance Structure

Estimation Method

Residual Variance Method

Fixed Effects SE Method

Degrees of Freedom Method
WORK. TOMSORT

PctDiff

Variance Components

REML

Profile

Prasad-Rao-Jeske-

Kackar-Harville

Kenward-Roger

Class Level Information

$\begin{array}{lllllll}\text { Class } & \text { Levels } & \text { Values } \\ \text { MDate } & 21 & 19281 & 19290 & 19318 & 19322 & 19331 \\ & & 19333 & 19336 & 19340 & 19341 & 19350 \\ & 19351 & 19352 & 19370 & 19371 & 19372 \\ & 19380 & 19381 & 19384 & 19386 & 19390 \\ & & 19398 & & & & \end{array}$

Dimensions

Covariance Parameters

2

Columns in $X$

Columns in $Z$

Subjects

Max Obs Per Subject

Observations Used

Observations Not Used

1

Total Observations

86

0

86

Iteration History

Iteration

Evaluations

-2 Res Log Like

Criterion

0

342.39709134

266.04482118

0.00022572

266.03188650

0.00000108

266.03182726

0.00000000

Convergence criteria met. 
SRNL-STI-2013-00139

Revision 0

The SAS System

09:55 Thursday, March 14, 2013

33

Analyte $=$ TOC RefValue $=20$

The Mixed Procedure

Covariance Parameter Estimates

$\begin{array}{lrrrr}\text { Cov Parm } & \text { Estimate } & \text { Alpha } & \text { Lower } & \text { Upper } \\ \text { MDate } & 3.0349 & 0.05 & 0.9865 & 5.0832 \\ \text { Residual } & 0.6316 & 0.05 & 0.4592 & 0.9236\end{array}$

Asymptotic Covariance Matrix of Estimates

$\begin{array}{rlrr}\text { Row } & \text { Cov Parm } & \text { CovP1 } & \text { CovP2 } \\ & & & \\ 1 & \text { MDate } & 1.0922 & -0.00697 \\ 2 & \text { Residual } & -0.00697 & 0.01248\end{array}$

\section{Fit Statistics}

$\begin{array}{ll}-2 \text { Res Log Likelihood } & 266.0 \\ \text { AIC (smaller is better) } & 270.0 \\ \text { AICC (smaller is better) } & 270.2 \\ \text { BIC (smaller is better) } & 272.1\end{array}$

Null Model Likelihood Ratio Test

$$
\begin{aligned}
& \text { DF Chi-Square } \quad \mathrm{Pr}>\mathrm{ChiSq} \\
& 1 \quad 76.37<.0001
\end{aligned}
$$

Solution for Fixed Effects

$\begin{array}{lrrrrr} & \text { Estimate } & \begin{array}{r}\text { Standard } \\ \text { Error }\end{array} & \text { DF } & t \text { Value } & \text { Pr }>|t| \\ \text { Intercept } & -0.1375 & 0.3912 & 18.9 & -0.35 & 0.7290\end{array}$

\begin{tabular}{|c|c|c|c|c|c|c|}
\hline Effect & MDate & Estimate & Pred & DF & t Value & $\operatorname{Pr}>|t|$ \\
\hline MDate & 19281 & -3.2041 & 0.6470 & 68.6 & -4.95 & $<.0001$ \\
\hline MDate & 19290 & -3.4868 & 0.8054 & 75.6 & -4.33 & $<.0001$ \\
\hline MDate & 19318 & -0.6297 & 0.5390 & 52.5 & -1.17 & 0.2479 \\
\hline MDate & 19322 & -0.6653 & 0.5390 & 52.5 & -1.23 & 0.2225 \\
\hline MDate & 19331 & 0.7605 & 0.5390 & 52.5 & 1.41 & 0.1641 \\
\hline
\end{tabular}

Solution for Random Effects 
SRNL-STI-2013-00139

Revision 0

The SAS System

09:55 Thursday, March 14, 2013

Analyte $=T O C$ RefValue $=20$

The Mixed Procedure

Solution for Random Effects

$\begin{array}{lrrrrrr}\text { Effect } & \text { MDate } & \text { Estimate } & \begin{array}{r}\text { Std Err } \\ \text { Pred }\end{array} & \text { DF } & \text { t Value } & \text { Pr }>|t| \\ \text { MDate } & 19333 & 1.5209 & 0.5390 & 52.5 & 2.82 & 0.0067 \\ \text { MDate } & 19336 & 0.4159 & 0.5390 & 52.5 & 0.77 & 0.4438 \\ \text { MDate } & 19340 & -0.6297 & 0.5390 & 52.5 & -1.17 & 0.2479 \\ \text { MDate } & 19341 & -0.4158 & 0.5390 & 52.5 & -0.77 & 0.4439 \\ \text { MDate } & 19350 & 0.1426 & 0.5390 & 52.5 & 0.26 & 0.7923 \\ \text { MDate } & 19351 & -0.2614 & 0.5390 & 52.5 & -0.48 & 0.6297 \\ \text { MDate } & 19352 & -1.2357 & 0.5390 & 52.5 & -2.29 & 0.0259 \\ \text { MDate } & 19370 & 1.0932 & 0.5390 & 52.5 & 2.03 & 0.0476 \\ \text { MDate } & 19371 & 2.6140 & 0.5390 & 52.5 & 4.85 & <.0001 \\ \text { MDate } & 19372 & 3.2897 & 0.4824 & 39.7 & 6.82 & <.0001 \\ \text { MDate } & 19380 & -0.4158 & 0.5390 & 52.5 & -0.77 & 0.4439 \\ \text { MDate } & 19381 & 1.4972 & 0.5390 & 52.5 & 2.78 & 0.0076 \\ \text { MDate } & 19384 & -0.1307 & 0.5390 & 52.5 & -0.24 & 0.8094 \\ \text { MDate } & 19386 & -2.3050 & 0.5390 & 52.5 & -4.28 & <.0001 \\ \text { MDate } & 19390 & 1.2854 & 0.4722 & 37.3 & 2.72 & 0.0098 \\ \text { MDate } & 19398 & 0.7605 & 0.5390 & 52.5 & 1.41 & 0.1641\end{array}$

The SAS System

09:55 Thursday, March 14, 201335

Analyte $=$ Tot0 $x$ RefValue $=2$

The Mixed Procedure

Model Information

Data Set

Dependent Variable

Covariance Structure

Estimation Method

Residual Variance Method

Fixed Effects SE Method

Degrees of Freedom Method
WORK. TOMSORT

PctDiff

Variance Components

REML

Profile

Prasad-Rao-Jeske-

Kackar-Harville

Kenward-Roger

Class Level Information

$\begin{array}{lllllll}\text { Class } & \text { Levels } & \text { Values } & & & \\ \text { MDate } & 89 & 19298 & 19300 & 19302 & 19304 & 19305 \\ & & 19306 & 19307 & 19310 & 19311 & 19312 \\ & 19313 & 19314 & 19315 & 19316 & 19317 \\ & 19318 & 19319 & 19321 & 19322 & 19323 \\ & 19324 & 19325 & 19326 & 19327 & 19329 \\ & 19330 & 19331 & 19333 & 19334 & 19335 \\ & 19336 & 19337 & 19338 & 19339 & 19340\end{array}$


SRNL-STI-2013-00139

Revision 0

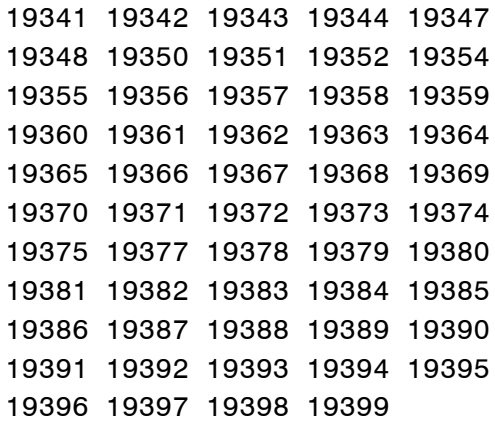

Dimensions

Covariance Parameters

Columns in $X$

2

Columns in $Z$

Subjects

Max Obs Per Subject

Observations Used

Observations Not Used

Total Observations

The SAS System

Analyte $=$ Tot0 $x$ RefValue $=2$

The Mixed Procedure

Iteration History

$\begin{array}{rrrr}\text { Iteration } & \text { Evaluations } & -2 \text { Res Log Like } & \text { Criterion } \\ & & & \\ 1 & 1 & 808.33464356 & \\ 2 & 2 & 730.92958405 & 0.00204489 \\ 3 & 1 & 730.38112210 & 0.00009731 \\ 4 & 1 & 730.35716408 & 0.00000026 \\ & 1 & 730.35710310 & 0.00000000\end{array}$

Convergence criteria met.

Covariance Parameter Estimates

$\begin{array}{lrrrr}\text { Cov Parm } & \text { Estimate } & \text { Alpha } & \text { Lower } & \text { Upper } \\ & & & & \\ \text { MDate } & 16.9584 & 0.05 & 11.3561 & 22.5608 \\ \text { Residual } & 2.5001 & 0.05 & 1.7538 & 3.8517\end{array}$

Asymptotic Covariance Matrix of Estimates

$\begin{array}{cccc}\text { Row } & \text { Cov Parm } & \text { CovP1 } & \text { CovP2 } \\ 1 & \text { MDate } & 8.1704 & -0.1766\end{array}$


SRNL-STI-2013-00139

Revision 0

2 Residual $\quad-0.1766 \quad 0.2469$

Fit Statistics

$\begin{array}{ll}\text {-2 Res Log Likelihood } & 730.4 \\ \text { AIC (smaller is better) } & 734.4 \\ \text { AICC (smaller is better) } & 734.4 \\ \text { BIC (smaller is better) } & 739.3\end{array}$

Null Model Likelihood Ratio Test

DF Chi-Square $\quad \mathrm{Pr}>\mathrm{ChiSq}$

$1 \quad 77.98 \quad<.0001$

The SAS System

09:55 Thursday, March 14, 201337

- Analyte=Tot0x RefValue $=2$

The Mixed Procedure

Solution for Fixed Effects

$\begin{array}{lrrrrr}\text { Effect } & \text { Estimate } & \begin{array}{r}\text { Standard } \\ \text { Error }\end{array} & \text { DF } & t \text { Value } & \operatorname{Pr}>|t| \\ \text { Intercept } & 4.1162 & 0.4606 & 88.4 & 8.94 & <.0001\end{array}$

Solution for Random Effects

$\begin{array}{lrrrrrr}\text { Effect } & \text { MDate } & \text { Estimate } & \begin{array}{r}\text { Std Err } \\ \text { Pred }\end{array} & \text { DF } & \text { t Value } & \text { Pr }>|t| \\ \text { MDate } & 19298 & -1.4086 & 1.5416 & 79.6 & -0.91 & 0.3637 \\ \text { MDate } & 19300 & 1.6418 & 1.5416 & 79.6 & 1.06 & 0.2901 \\ \text { MDate } & 19302 & -0.1013 & 1.5416 & 79.6 & -0.07 & 0.9478 \\ \text { MDate } & 19304 & -3.1516 & 1.5416 & 79.6 & -2.04 & 0.0442 \\ \text { MDate } & 19305 & -1.8443 & 1.5416 & 79.6 & -1.20 & 0.2351 \\ \text { MDate } & 19306 & -2.2801 & 1.5416 & 79.6 & -1.48 & 0.1431 \\ \text { MDate } & 19307 & -1.8443 & 1.5416 & 79.6 & -1.20 & 0.2351 \\ \text { MDate } & 19310 & 0.7702 & 1.5416 & 79.6 & 0.50 & 0.6187 \\ \text { MDate } & 19311 & 0.7702 & 1.5416 & 79.6 & 0.50 & 0.6187 \\ \text { MDate } & 19312 & -0.1013 & 1.5416 & 79.6 & -0.07 & 0.9478 \\ \text { MDate } & 19313 & 0.3574 & 1.1660 & 79.9 & 0.31 & 0.7600 \\ \text { MDate } & 19314 & 0.7702 & 1.5416 & 79.6 & 0.50 & 0.6187 \\ \text { MDate } & 19315 & 0.3345 & 1.5416 & 79.6 & 0.22 & 0.8288 \\ \text { MDate } & 19316 & -0.9728 & 1.5416 & 79.6 & -0.63 & 0.5298 \\ \text { MDate } & 19317 & -0.9728 & 1.5416 & 79.6 & -0.63 & 0.5298 \\ \text { MDate } & 19318 & -0.1082 & 1.1660 & 79.9 & -0.09 & 0.9263 \\ \text { MDate } & 19319 & 0.3345 & 1.5416 & 79.6 & 0.22 & 0.8288 \\ \text { MDate } & 19321 & 0.8424 & 0.9963 & 85.6 & 0.85 & 0.4002 \\ \text { MDate } & 19322 & 0.3345 & 1.5416 & 79.6 & 0.22 & 0.8288\end{array}$


SRNL-STI-2013-00139

Revision 0

$\begin{array}{lrrrrrr}\text { MDate } & 19323 & 0.3574 & 1.1660 & 79.9 & 0.31 & 0.7600 \\ \text { MDate } & 19324 & 0.7702 & 1.5416 & 79.6 & 0.50 & 0.6187 \\ \text { MDate } & 19325 & 0.5247 & 0.9963 & 85.6 & 0.53 & 0.5998 \\ \text { MDate } & 19326 & -0.5370 & 1.5416 & 79.6 & -0.35 & 0.7285 \\ \text { MDate } & 19327 & 0.3345 & 1.5416 & 79.6 & 0.22 & 0.8288 \\ \text { MDate } & 19329 & 1.9873 & 1.1660 & 79.9 & 1.70 & 0.0922 \\ \text { MDate } & 19330 & 2.4530 & 1.1660 & 79.9 & 2.10 & 0.0386 \\ \text { MDate } & 19331 & 0.3345 & 1.5416 & 79.6 & 0.22 & 0.8288 \\ \text { MDate } & 19333 & 1.9873 & 1.1660 & 79.9 & 1.70 & 0.0922 \\ \text { MDate } & 19334 & 2.0775 & 1.5416 & 79.6 & 1.35 & 0.1816 \\ \text { MDate } & 19335 & -0.1013 & 1.5416 & 79.6 & -0.07 & 0.9478 \\ \text { MDate } & 19336 & -0.5370 & 1.5416 & 79.6 & -0.35 & 0.7285 \\ \text { MDate } & 19337 & 2.5133 & 1.5416 & 79.6 & 1.63 & 0.1070 \\ \text { MDate } & 19338 & -0.5370 & 1.5416 & 79.6 & -0.35 & 0.7285 \\ \text { MDate } & 19339 & 0.5903 & 1.1660 & 79.9 & 0.51 & 0.6141 \\ \text { MDate } & 19340 & -0.8067 & 1.1660 & 79.9 & -0.69 & 0.4910 \\ \text { MDate } & 19341 & -6.4652 & 0.9963 & 85.6 & -6.49 & <.0001\end{array}$

The SAS System

09:55 Thursday, March 14, 201338

Analyte=Tot0x RefValue $=2$

The Mixed Procedure

Solution for Random Effects

$\begin{array}{lcccccc}\text { Effect } & \text { MDate } & \text { Estimate } & \text { Std Err } & & \\ \text { MDate } & 19342 & -5.9886 & 0.9963 & 85.6 & -6.01 & <.0001 \\ \text { MDate } & 19343 & -4.4589 & 1.5416 & 79.6 & -2.89 & 0.0049 \\ \text { MDate } & 19344 & -4.0231 & 1.5416 & 79.6 & -2.61 & 0.0108 \\ \text { MDate } & 19347 & -1.7381 & 1.1660 & 79.9 & -1.49 & 0.1400 \\ \text { MDate } & 19348 & -2.4366 & 1.1660 & 79.9 & -2.09 & 0.0398 \\ \text { MDate } & 19350 & -3.6057 & 0.9963 & 85.6 & -3.62 & 0.0005 \\ \text { MDate } & 19351 & -8.3807 & 1.5416 & 79.6 & -5.44 & <.0001 \\ \text { MDate } & 19352 & -7.5092 & 1.5416 & 79.6 & -4.87 & <.0001 \\ \text { MDate } & 19354 & -3.1516 & 1.5416 & 79.6 & -2.04 & 0.0442 \\ \text { MDate } & 19355 & -2.7158 & 1.5416 & 79.6 & -1.76 & 0.0820 \\ \text { MDate } & 19356 & -2.7158 & 1.5416 & 79.6 & -1.76 & 0.0820 \\ \text { MDate } & 19357 & -2.7158 & 1.5416 & 79.6 & -1.76 & 0.0820 \\ \text { MDate } & 19358 & -3.1351 & 1.1660 & 79.9 & -2.69 & 0.0087 \\ \text { MDate } & 19359 & -2.9023 & 1.1660 & 79.9 & -2.49 & 0.0149 \\ \text { MDate } & 19360 & -2.7158 & 1.5416 & 79.6 & -1.76 & 0.0820 \\ \text { MDate } & 19361 & -4.2993 & 1.1660 & 79.9 & -3.69 & 0.0004 \\ \text { MDate } & 19362 & -3.1516 & 1.5416 & 79.6 & -2.04 & 0.0442 \\ \text { MDate } & 19363 & -4.5321 & 1.1660 & 79.9 & -3.89 & 0.0002 \\ \text { MDate } & 19364 & -4.0231 & 1.5416 & 79.6 & -2.61 & 0.0108 \\ \text { MDate } & 19365 & -4.0231 & 1.5416 & 79.6 & -2.61 & 0.0108 \\ \text { MDate } & 19366 & 4.5485 & 1.1660 & 79.9 & 3.90 & 0.0002 \\ \text { MDate } & 19367 & 5.5636 & 1.5416 & 79.6 & 3.61 & 0.0005 \\ \text { MDate } & 19368 & 6.4351 & 1.5416 & 79.6 & 4.17 & <.0001 \\ \text { MDate } & 19369 & 2.9186 & 1.1660 & 79.9 & 2.50 & 0.0144 \\ \text { MDate } & 19370 & 0.3345 & 1.5416 & 79.6 & 0.22 & 0.8288 \\ \text { MDate } & 19371 & 3.2253 & 0.9963 & 85.6 & 3.24 & 0.0017 \\ \text { MDate } & 19372 & 5.1924 & 0.8963 & 92.3 & 5.79 & <.0001 \\ \text { MDate } & 19373 & 8.6139 & 1.5416 & 79.6 & 5.59 & <.0001\end{array}$


SRNL-STI-2013-00139

Revision 0

$\begin{array}{lrrrrrr}\text { MDate } & 19374 & 8.1781 & 1.5416 & 79.6 & 5.30 & <.0001 \\ \text { MDate } & 19375 & 8.7396 & 1.1660 & 79.9 & 7.50 & <.0001 \\ \text { MDate } & 19377 & 8.0411 & 1.1660 & 79.9 & 6.90 & <.0001 \\ \text { MDate } & 19378 & 8.6139 & 1.5416 & 79.6 & 5.59 & <.0001 \\ \text { MDate } & 19379 & 7.3066 & 1.5416 & 79.6 & 4.74 & <.0001 \\ \text { MDate } & 19380 & 10.1366 & 1.1660 & 79.9 & 8.69 & <.0001 \\ \text { MDate } & 19381 & 8.1781 & 1.5416 & 79.6 & 5.30 & <.0001 \\ \text { MDate } & 19382 & 9.2052 & 1.1660 & 79.9 & 7.89 & <.0001 \\ \text { MDate } & 19383 & -1.0396 & 1.1660 & 79.9 & -0.89 & 0.3753 \\ \text { MDate } & 19384 & -3.8040 & 0.8295 & 99.1 & -4.59 & <.0001 \\ \text { MDate } & 19385 & -3.1516 & 1.5416 & 79.6 & -2.04 & 0.0442 \\ \text { MDate } & 19386 & -3.4468 & 0.9963 & 85.6 & -3.46 & 0.0008 \\ \text { MDate } & 19387 & -0.1013 & 1.5416 & 79.6 & -0.07 & 0.9478 \\ \text { MDate } & 19388 & -0.9728 & 1.5416 & 79.6 & -0.63 & 0.5298 \\ \text { MDate } & 19389 & -3.1516 & 1.5416 & 79.6 & -2.04 & 0.0442 \\ \text { MDate } & 19390 & -3.0054 & 0.8963 & 92.3 & -3.35 & 0.0012\end{array}$

The SAS System

09:55 Thursday, March 14, 201339

Analyte $=$ Tot0 $x$ RefValue $=2$

The Mixed Procedure

Solution for Random Effects

$\begin{array}{lrrrrrr}\text { Effect } & \text { MDate } & \text { Estimate } & \begin{array}{r}\text { Std Err } \\ \text { Pred }\end{array} & \text { DF } & t \text { Value } & \operatorname{Pr~}>|t| \\ \text { MDate } & 19391 & -1.4086 & 1.5416 & 79.6 & -0.91 & 0.3637 \\ \text { MDate } & 19392 & -0.9728 & 1.5416 & 79.6 & -0.63 & 0.5298 \\ \text { MDate } & 19393 & -0.5739 & 1.1660 & 79.9 & -0.49 & 0.6239 \\ \text { MDate } & 19394 & -0.3411 & 1.1660 & 79.9 & -0.29 & 0.7707 \\ \text { MDate } & 19395 & 1.6418 & 1.5416 & 79.6 & 1.06 & 0.2901 \\ \text { MDate } & 19396 & 0.5903 & 1.1660 & 79.9 & 0.51 & 0.6141 \\ \text { MDate } & 19397 & -2.7158 & 1.5416 & 79.6 & -1.76 & 0.0820 \\ \text { MDate } & 19398 & -2.3348 & 0.9963 & 85.6 & -2.34 & 0.0214 \\ \text { MDate } & 19399 & -0.5370 & 1.5416 & 79.6 & -0.35 & 0.7285\end{array}$

The SAS System

09:55 Thursday, March 14, 201340

Analyte $=$ Tot0 $x$ RefValue $=16$

The Mixed Procedure

Model Information

Data Set

Dependent Variable

Covariance Structure

Estimation Method

Residual Variance Method

Fixed Effects SE Method

Degrees of Freedom Method
WORK. TOMSORT

PctDiff

Variance Components

REML

Profile

Prasad-Rao-Jeske-

Kackar-Harville

Kenward-Roger

Class Level Information 
SRNL-STI-2013-00139

Revision 0

\begin{tabular}{|c|c|c|c|c|c|}
\hline Class & Levels & Values & & & \\
\hline MDate & 83 & 1929819300 & 19302 & 19304 & 19305 \\
\hline & & $19306 \quad 19307$ & 19310 & 19311 & 19312 \\
\hline & & 1931319314 & 19315 & 19316 & 19317 \\
\hline & & 1931819319 & 19321 & 19322 & 19323 \\
\hline & & 1932419325 & 19326 & 19327 & 19329 \\
\hline & & 1933019331 & 19333 & 19334 & 19335 \\
\hline & & 1933619337 & 19338 & 19339 & 19340 \\
\hline & & 1934119342 & 19343 & 19344 & 19347 \\
\hline & & 1934819350 & 19354 & 19355 & 19356 \\
\hline & & 1935719358 & 19359 & 19360 & 19361 \\
\hline & & 1936219363 & 19364 & 19365 & 19366 \\
\hline & & 1936719368 & 19369 & 19370 & 19371 \\
\hline & & 1937219373 & 19374 & 19375 & 19377 \\
\hline & & 1937919380 & 19383 & 19384 & 19385 \\
\hline & & 1938619387 & 19389 & 19390 & 19391 \\
\hline & & 1939219393 & 19394 & 19395 & 19396 \\
\hline & & 1939719398 & 19399 & & \\
\hline
\end{tabular}

Dimensions

$\begin{array}{lr}\text { Covariance Parameters } & 2 \\ \text { Columns in X } & 1 \\ \text { Columns in Z } & 83 \\ \text { Subjects } & 1 \\ \text { Max Obs Per Subject } & 130 \\ \text { Observations Used } & 130 \\ \text { Observations Not Used } & 0 \\ \text { Total Observations } & 130\end{array}$

The SAS System

09:55 Thursday, March 14, 2013

Analyte=Tot0x RefValue $=16$

The Mixed Procedure

Iteration History

$\begin{array}{rrrr}\text { Iteration } & \text { Evaluations } & -2 \text { Res Log Like } & \text { Criterion } \\ 0 & 1 & 624.26262434 & \\ 1 & 2 & 594.98459651 & 0.00000126 \\ 2 & 1 & 594.98436971 & 0.00000000\end{array}$

Convergence criteria met.

Covariance Parameter Estimates

$\begin{array}{lrrrr}\text { Cov Parm } & \text { Estimate } & \text { Alpha } & \text { Lower } & \text { Upper } \\ & & & & \\ \text { MDate } & 4.6863 & 0.05 & 2.6092 & 6.7635 \\ \text { Residual } & 2.4473 & 0.05 & 1.7066 & 3.8038\end{array}$


SRNL-STI-2013-00139

Revision 0

Asymptotic Covariance Matrix of Estimates

$\begin{array}{rlrr}\text { Row } & \text { Cov Parm } & \text { CovP1 } & \text { CovP2 } \\ & & & \\ 1 & \text { MDate } & 1.1231 & -0.1677 \\ 2 & \text { Residual } & -0.1677 & 0.2454\end{array}$

Fit Statistics

$\begin{array}{ll}-2 \text { Res Log Likelihood } & 595.0 \\ \text { AIC (smaller is better) } & 599.0 \\ \text { AICC (smaller is better) } & 599.1 \\ \text { BIC (smaller is better) } & 603.8\end{array}$

Null Model Likelihood Ratio Test

$$
\begin{aligned}
& \text { DF Chi-Square } \quad \mathrm{Pr}>\mathrm{ChiSq} \\
& 1 \quad 29.28<.0001
\end{aligned}
$$

The SAS System

09:55 Thursday, March 14, 201342

Analyte $=$ Tot0 $x$ RefValue $=16$

The Mixed Procedure

Solution for Fixed Effects

$\begin{array}{lrrrrr} & \text { Estimate } & \begin{array}{r}\text { Standard } \\ \text { Erfor }\end{array} & \text { DF } & t \text { Value } & \operatorname{Pr}>|t| \\ \text { Intercept } & -0.01466 & 0.2801 & 81.8 & -0.05 & 0.9584\end{array}$

Solution for Random Effects

$\begin{array}{lcccccc}\text { Effect } & \text { MDate } & \text { Estimate } & \begin{array}{c}\text { Std Err } \\ \text { Pred }\end{array} & \text { DF } & t \text { Value } & \text { Pr }>|t| \\ \text { MDate } & 19298 & -0.4831 & 1.3146 & 121 & -0.37 & 0.7139 \\ \text { MDate } & 19300 & -0.4420 & 1.3146 & 121 & -0.34 & 0.7373 \\ \text { MDate } & 19302 & -0.1546 & 1.3146 & 121 & -0.12 & 0.9066 \\ \text { MDate } & 19304 & -1.3453 & 1.3146 & 121 & -1.02 & 0.3082 \\ \text { MDate } & 19305 & -0.8526 & 1.3146 & 121 & -0.65 & 0.5178 \\ \text { MDate } & 19306 & -0.9347 & 1.3146 & 121 & -0.71 & 0.4784 \\ \text { MDate } & 19307 & -0.9347 & 1.3146 & 121 & -0.71 & 0.4784 \\ \text { MDate } & 19310 & -0.7705 & 1.3146 & 121 & -0.59 & 0.5589 \\ \text { MDate } & 19311 & -0.6062 & 1.3146 & 121 & -0.46 & 0.6455 \\ \text { MDate } & 19312 & -0.9347 & 1.3146 & 121 & -0.71 & 0.4784 \\ \text { MDate } & 19313 & 0.4329 & 1.0286 & 96.9 & 0.42 & 0.6748 \\ \text { MDate } & 19314 & 1.3646 & 1.3146 & 121 & 1.04 & 0.3013\end{array}$


SRNL-STI-2013-00139

Revision 0

$\begin{array}{lrrrrrr}\text { MDate } & 19315 & -0.3188 & 1.3146 & 121 & -0.24 & 0.8088 \\ \text { MDate } & 19316 & -0.8937 & 1.3146 & 121 & -0.68 & 0.4979 \\ \text { MDate } & 19317 & 1.8162 & 1.3146 & 121 & 1.38 & 0.1696 \\ \text { MDate } & 19318 & -1.4256 & 1.0286 & 96.9 & -1.39 & 0.1690 \\ \text { MDate } & 19319 & -0.4420 & 1.3146 & 121 & -0.34 & 0.7373 \\ \text { MDate } & 19321 & 1.2014 & 0.8793 & 88.1 & 1.37 & 0.1753 \\ \text { MDate } & 19322 & 1.8573 & 1.3146 & 121 & 1.41 & 0.1603 \\ \text { MDate } & 19323 & -1.4752 & 1.0286 & 96.9 & -1.43 & 0.1548 \\ \text { MDate } & 19324 & 0.2149 & 1.3146 & 121 & 0.16 & 0.8704 \\ \text { MDate } & 19325 & -0.1650 & 0.8793 & 88.1 & -0.19 & 0.8516 \\ \text { MDate } & 19326 & 0.2560 & 1.3146 & 121 & 0.19 & 0.8459 \\ \text { MDate } & 19327 & -2.4539 & 1.3146 & 121 & -1.87 & 0.0644 \\ \text { MDate } & 19329 & 0.1355 & 1.0286 & 96.9 & 0.13 & 0.8955 \\ \text { MDate } & 19330 & 2.2170 & 1.0286 & 96.9 & 2.16 & 0.0336 \\ \text { MDate } & 19331 & -1.2632 & 1.3146 & 121 & -0.96 & 0.3385 \\ \text { MDate } & 19333 & 1.9197 & 1.0286 & 96.9 & 1.87 & 0.0650 \\ \text { MDate } & 19334 & 0.2149 & 1.3146 & 121 & 0.16 & 0.8704 \\ \text { MDate } & 19335 & -0.7294 & 1.3146 & 121 & -0.55 & 0.5800 \\ \text { MDate } & 19336 & -0.1135 & 1.3146 & 121 & -0.09 & 0.9313 \\ \text { MDate } & 19337 & -0.6062 & 1.3146 & 121 & -0.46 & 0.6455 \\ \text { MDate } & 19338 & 0.009634 & 1.3146 & 121 & 0.01 & 0.9942 \\ \text { MDate } & 19339 & -0.2114 & 1.0286 & 96.9 & -0.21 & 0.8376 \\ \text { MDate } & 19340 & -0.01315 & 1.0286 & 96.9 & -0.01 & 0.9898 \\ \text { MDate } & 19341 & -0.6263 & 0.8793 & 88.1 & -0.71 & 0.4782\end{array}$

The SAS System

09:55 Thursday, March 14, 201343

Analyte $=$ Tot0x RefValue $=16$

The Mixed Procedure

Solution for Random Effects

$\begin{array}{lrrrrrr}\text { Effect } & \text { MDate } & \text { Estimate } & \text { Std Err } & & \\ \text { MDate } & 19342 & 0.03023 & 0.8793 & 88.1 & 0.03 & 0.9726 \\ \text { MDate } & 19343 & 0.09175 & 1.3146 & 121 & 0.07 & 0.9445 \\ \text { MDate } & 19344 & 0.09175 & 1.3146 & 121 & 0.07 & 0.9445 \\ \text { MDate } & 19347 & 1.4984 & 1.0286 & 96.9 & 1.46 & 0.1484 \\ \text { MDate } & 19348 & -0.7813 & 1.0286 & 96.9 & -0.76 & 0.4494 \\ \text { MDate } & 19350 & -1.2769 & 1.0286 & 96.9 & -1.24 & 0.2175 \\ \text { MDate } & 19354 & -0.03143 & 1.3146 & 121 & -0.02 & 0.9810 \\ \text { MDate } & 19355 & 0.1328 & 1.3146 & 121 & 0.10 & 0.9197 \\ \text { MDate } & 19356 & 0.1739 & 1.3146 & 121 & 0.13 & 0.8950 \\ \text { MDate } & 19357 & 0.4202 & 1.3146 & 121 & 0.32 & 0.7498 \\ \text { MDate } & 19358 & 0.3833 & 1.0286 & 96.9 & 0.37 & 0.7102 \\ \text { MDate } & 19359 & 0.5816 & 1.0286 & 96.9 & 0.57 & 0.5731 \\ \text { MDate } & 19360 & 0.5434 & 1.3146 & 121 & 0.41 & 0.6801 \\ \text { MDate } & 19361 & -0.8804 & 1.0286 & 96.9 & -0.86 & 0.3941 \\ \text { MDate } & 19362 & -1.1400 & 1.3146 & 121 & -0.87 & 0.3876 \\ \text { MDate } & 19363 & -0.8309 & 1.0286 & 96.9 & -0.81 & 0.4212 \\ \text { MDate } & 19364 & -0.7705 & 1.3146 & 121 & -0.59 & 0.5589 \\ \text { MDate } & 19365 & -0.03143 & 1.3146 & 121 & -0.02 & 0.9810 \\ \text { MDate } & 19366 & -0.1866 & 1.0286 & 96.9 & -0.18 & 0.8564 \\ \text { MDate } & 19367 & 3.0480 & 1.3146 & 121 & 2.32 & 0.0221 \\ \text { MDate } & 19368 & -0.3599 & 1.3146 & 121 & -0.27 & 0.7847\end{array}$


SRNL-STI-2013-00139

Revision 0

$\begin{array}{lrrrrrr}\text { MDate } & 19369 & 2.2418 & 1.0286 & 96.9 & 2.18 & 0.0317 \\ \text { MDate } & 19370 & 3.1711 & 1.3146 & 121 & 2.41 & 0.0174 \\ \text { MDate } & 19371 & 1.8047 & 0.8793 & 88.1 & 2.05 & 0.0431 \\ \text { MDate } & 19372 & 3.5614 & 0.8793 & 88.1 & 4.05 & 0.0001 \\ \text { MDate } & 19373 & 3.4175 & 1.3146 & 121 & 2.60 & 0.0105 \\ \text { MDate } & 19374 & 3.7038 & 1.0286 & 96.9 & 3.60 & 0.0005 \\ \text { MDate } & 19375 & 4.2985 & 1.0286 & 96.9 & 4.18 & <.0001 \\ \text { MDate } & 19377 & 3.5817 & 1.3146 & 121 & 2.72 & 0.0074 \\ \text { MDate } & 19379 & 3.7460 & 1.3146 & 121 & 2.85 & 0.0051 \\ \text { MDate } & 19380 & 5.5623 & 1.0286 & 96.9 & 5.41 & <.0001 \\ \text { MDate } & 19383 & 0.7550 & 1.0286 & 96.9 & 0.73 & 0.4647 \\ \text { MDate } & 19384 & -0.08858 & 0.7191 & 84.5 & -0.12 & 0.9022 \\ \text { MDate } & 19385 & 0.1328 & 1.3146 & 121 & 0.10 & 0.9197 \\ \text { MDate } & 19386 & 0.08347 & 0.8793 & 88.1 & 0.09 & 0.9246 \\ \text { MDate } & 19387 & -1.9612 & 1.3146 & 121 & -1.49 & 0.1383 \\ \text { MDate } & 19389 & -4.0962 & 1.3146 & 121 & -3.12 & 0.0023 \\ \text { MDate } & 19390 & -2.9585 & 0.7850 & 85.1 & -3.77 & 0.0003 \\ \text { MDate } & 19391 & -3.5214 & 1.3146 & 121 & -2.68 & 0.0084 \\ \text { MDate } & 19392 & -1.6327 & 1.3146 & 121 & -1.24 & 0.2166 \\ \text { MDate } & 19393 & -1.8469 & 1.0286 & 96.9 & -1.80 & 0.0757 \\ \text { MDate } & 19394 & -2.9867 & 1.0286 & 96.9 & -2.90 & 0.0046 \\ \text { MDate } & 19395 & -3.5214 & 1.3146 & 121 & -2.68 & 0.0084 \\ \text { MDate } & 19396 & -1.2274 & 1.0286 & 96.9 & -1.19 & 0.2357\end{array}$

The SAS System

09:55 Thursday, March 14, 201344

Analyte=Tot0x RefValue $=16$

The Mixed Procedure

Solution for Random Effects

$\begin{array}{lrrrrrr}\text { Effect } & \text { MDate } & \text { Estimate } & \begin{array}{r}\text { Std Err } \\ \text { Pred }\end{array} & \text { DF } & \text { t Value } & \operatorname{Pr}>|t| \\ \text { MDate } & 19397 & -1.7559 & 1.3146 & 121 & -1.34 & 0.1842 \\ \text { MDate } & 19398 & -3.0218 & 0.8793 & 88.1 & -3.44 & 0.0009 \\ \text { MDate } & 19399 & -1.5917 & 1.3146 & 121 & -1.21 & 0.2283\end{array}$




\section{Distribution:}

\begin{tabular}{|l|l|}
\hline Name: & Location: \\
\hline Sharon Marra & $773-\mathrm{A}$ \\
\hline Connie Herman & $999-\mathrm{W}$ \\
\hline Charles J. Coleman & $773-\mathrm{A}$ \\
\hline Clint Gregory & $773-\mathrm{A}$ \\
\hline Mark Barnes & $773-\mathrm{A}$ \\
\hline Patricia Lee & $703-41 \mathrm{~A}$ \\
\hline Gene Shine & $703-41 \mathrm{~A}$ \\
\hline Damon R. Click & $999-\mathrm{W}$ \\
\hline Boyd Wiedenman & $773-\mathrm{A}$ \\
\hline Tom White & $773-\mathrm{A}$ \\
\hline \hline Michael Stone & $999-\mathrm{W}$ \\
\hline David Peeler & $999-\mathrm{W}$ \\
\hline Tommy Edwards & $999-\mathrm{W}$ \\
\hline Alex Choi & $999-\mathrm{W}$ \\
\hline Gene Daniel & $999-\mathrm{W}$ \\
\hline Frank Smith & $703-41 \mathrm{~A}$ \\
\hline Shawn Tester & $704-27 \mathrm{~S}$ \\
\hline John Schwenker & $766-\mathrm{H}$ \\
\hline David Best & $999-\mathrm{W}$ \\
\hline Eric Freed & $704-\mathrm{S}$ \\
\hline Jonathan Bricker & $704-27 \mathrm{~S}$ \\
\hline
\end{tabular}

\begin{tabular}{|l|l|}
\hline Name: & Location: \\
\hline Dave Sherburne & $704-\mathrm{S}$ \\
\hline John Iaukea & $704-30 \mathrm{~S}$ \\
\hline Aaron Staub & $704-27 \mathrm{~S}$ \\
\hline Jeff Ray & $704-\mathrm{S}$ \\
\hline Robert Hinds & $704-\mathrm{S}$ \\
\hline Perry Bovan & $704-27 \mathrm{~S}$ \\
\hline Terri Fellinger & $704-26 \mathrm{~S}$ \\
\hline Ryan McNew & $704-\mathrm{S}$ \\
\hline Michael J. Hart & $210-\mathrm{S}$ \\
\hline Roger N. Mahannah & $704-28 \mathrm{~S}$ \\
\hline Michael T. Feller & $704-28 \mathrm{~S}$ \\
\hline Omar Cardona-Quiles & $704-24 \mathrm{~S}$ \\
\hline Amanda Shafer & $704-27 \mathrm{~S}$ \\
\hline Mason Clark & $704-27 \mathrm{~S}$ \\
\hline \hline Helen Pittman & $704-27 \mathrm{~S}$ \\
\hline Hank Elder & $704-24 \mathrm{~S}$ \\
\hline Bill Holtzscheiter & $704-15 \mathrm{~S}$ \\
\hline John Windham & $704-30 \mathrm{~S}$ \\
\hline Pat Vaughan & $773-41 \mathrm{~A}$ \\
\hline
\end{tabular}

